\title{
BROADBAND TECHNOLOGY ROADMAP FOR RURAL AREAS IN THE ANDES AND AMAZON REGIONS OF PERU \\ by DAVID ESPINOZA AGUILAR
}

M.S., University of Colorado at Boulder, 2011

B.S., Pontifical Catholic University of Peru, 2002

\author{
A thesis submitted to the \\ Faculty of the Graduate School of the \\ University of Colorado in partial fulfillment \\ of the requirement for the degree of \\ Doctor of Philosophy \\ Interdisciplinary Telecommunications Program
}


This thesis entitled:

Broadband Technology Roadmap for Rural Areas in the Andes and Amazon Regions of Peru written by David Espinoza Aguilar has been approved for the Interdisciplinary Telecommunications Program

Professor David Reed, Chair

Professor Douglas Sicker

Professor Martin Taschdjian

Professor Revital Sterling

Professor Thomas Schwengler

Professor Patrick Ryan

Date

The final copy of this thesis has been examined by the signatories, and we Find that both the content and the form meet acceptable presentation standards Of scholarly work in the above mentioned discipline. 
Espinoza Aguilar, David (Ph.D., Telecommunications)

Broadband Technology Roadmap for Rural Areas in the Andes and Amazon

Regions of Peru

Thesis directed by Professor David P. Reed

This dissertation addresses the research question: What is the broadband roadmap for introducing broadband services to unserved areas in the Andes and Amazon Regions of Peru? Detailed technical and engineering cost analyses are conducted for evaluating the deployment and operation of wireless broadband local access networks for a 10-year period in two scenarios in the Andes and Amazon regions of Peru, in the context of the Peruvian National Broadband Plan, using current wireless broadband technologies (Wi-Fi, WiMAX, LTE, TVWS) and new stratospheric platforms (super-pressure balloons). These analyses show that based on current spectrum policies LTE is the lowest cost option. Current available spectrum policies and alternatives to enable more spectrum for new access network deployments are also proposed. Finally, a technology broadband roadmap for the 10-year period is presented, which includes using stratospheric balloons (carrying micro-LTE base stations) for the first years, and deploying complementary terrestrial LTE networks for the rest of the 10-year period. 


\section{ACKNOWLEDGEMENTS}

First, I would like to express my gratitude to my advisor David Reed for his guidance and mentorship to conduct this interdisciplinary research, and also for his encouragement to develop soft skills to present the research in conferences and graduate student consortiums.

I would like to thank my committee members, Professor Douglas Sicker, Professor Taschdjian, Professor Revi Sterling, Professor Thomas Schwengler and Professor Patrick Ryan for their guidance on the different disciplines part of this research such as wireless network design, economics, regulation and policy, and social sciences.

I would also like to thank Professor Harvey Gates for his guidance on unmanned aerial systems and aeronautics field.

I also would like to thank Google for the Faculty Research Award, granted to Professor David Reed for this research, and ITP for the Teaching Fellowship which both together funded my Ph.D. studies.

I would like to thank the Investment Fund in Telecommunications (FITEL) of Peru for providing data and information regarding the Peruvian National Broadband Plan, and the deployment and operation of transport and wireless access networks across Peru under this plan.

I would like to thank academic and industry experts that provided feedback and suggestions to this research in meetings, conference calls, emails, and in 
conferences such as the Conference on Communications Information and Internet Policy (TPRC 2015) and the Technology, Management and Policy Graduate Student Consortium (TMP 2015 and TMP 2016).

Finally, I would like to thank my wife Sarah, for her every day support and kind encouragement words to navigate through my Ph.D. studies. I would also like to thank my parents Simon and Sara, and my sister Sofia for their encouragement along these years. 


\section{CONTENTS}

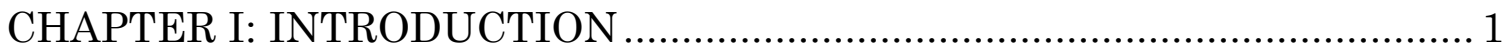

1.1 Statement of the problem and research question................................... 1

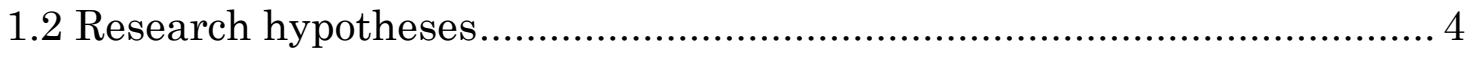

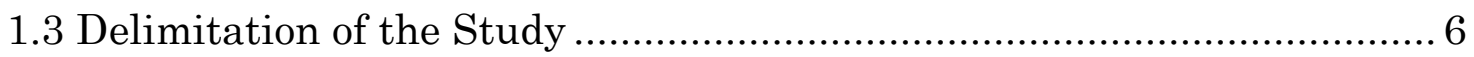

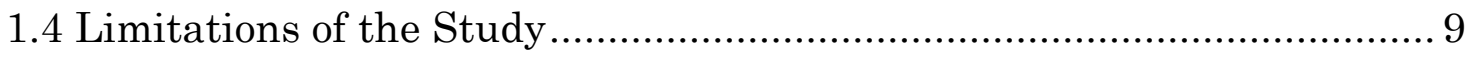

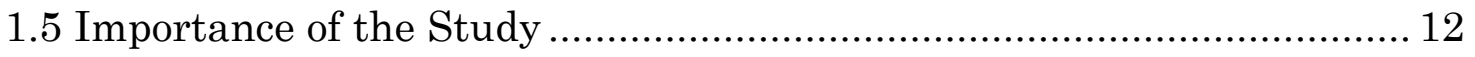

CHAPTER II: REVIEW OF THE LITERATURE...................................... 15

2.1 Broadband Landscape in Latin America and Peru .............................. 15

2.1.1 Broadband penetration in the Andes and Amazon regions in Peru ... 17

2.1.2 National broadband plan: backbone and access networks ............. 22

2.2 Wireless Broadband Technology Options for Rural Areas.....................26

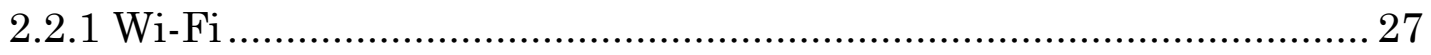

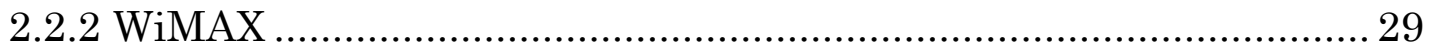

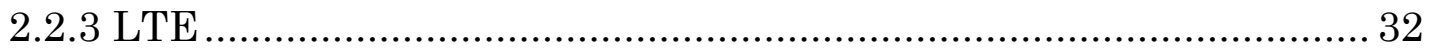

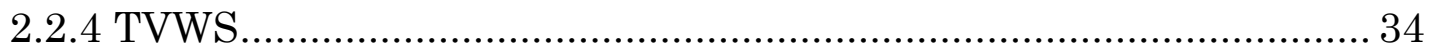

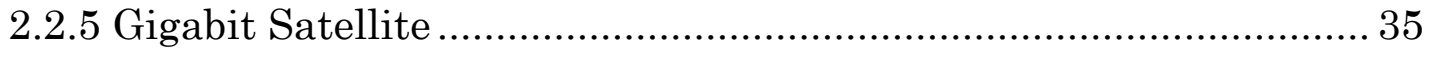

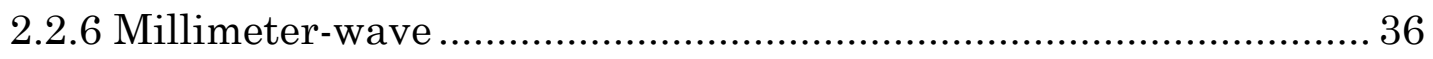

2.3 New unmanned aerial systems for stratospheric wireless access

networks. 
2.3.1 Unmanned super-pressure balloons: Project Loon 37

2.3.2 Unmanned aircrafts: Google and Facebook solar-powered drones. 39

2.4 Regulatory and Policy Environment for Broadband Services in Peru .. 40

2.4.1 National fiber optic backbone network and transport and access

networks 42

2.4.2 Spectrum regulation 45

CHAPTER III: RESEARCH METHODOLOGY ………………...................... 50

3.1 Broadband Service Demand Estimation............................................... 54

3.1.1 Selection of study areas and access network coverage ................... 54

3.1.2 Broadband residential customer and speed demand estimation for

a 10-year period

3.2 Technical analysis: wireless broadband access networks design and simulation in EDX 68

3.2.1 Propagation model for simulations 69

3.2.2 Environment simulation settings for the Andes and Amazon study

areas 71

3.2.3 RF equipment and network simulation settings ........................... 75

3.2.4 FITEL access network design requirements ................................. 77

3.2.5 Spectrum resources, reuse factor and overbooking ........................ 80

3.2.6 Wireless access network design and simulation in EDX 82 
3.3 Engineering cost analysis: access network deployment and operation cost model in Analytica

3.3.1 Engineering cost model design in Analytica 92

3.3.2 Cost data collection and processing 97 CHAPTER IV: TECHNICAL AND ENGINEERING COST ANALYSIS

RESULTS 104

4.1 WBLAN design and EDX simulation results - Yauli (Andes Region) and Samugari (Amazon Region) study areas 104

4.1.1 Current technologies: Wi-Fi, WiMAX, LTE and TVWS 104

4.1.2 Stratospheric Access Networks 119

4.2 WBLAN Engineering cost analysis results - Yauli (Andes Region) and Samugari (Amazon Region) study areas 128

4.2.1 Current Technologies: Wi-Fi, WiMAX, LTE and TVWS 128

4.2.2 Broadband service cost for the Yauli and Samugari area 140

4.3 Sensitivity analysis for current wireless technologies 146

4.3.1 CAPEX and OPEX categories 147

4.3.2 Wireless AP/BSs. 149

4.3.3 Tower Infrastructure 152

CHAPTER V: REGULATORY AND POLICY IMPLICATION \& BROADBAND TECHNOLOGY ROADMAP 156 
5.1 Regulatory and Policy Implications

5.1.1 Spectrum Proposed Spectrum Scenario: 1.7/2.1GHz LTE band using $40+40 \mathrm{MHz}$ spectrum 156

5.1.2 Government subsidy for introducing broadband services in rural

areas..... 171

5.1.3 Overbooking proposed scenario: Maintaining 1:10 oversubscription rate in rural areas 179

5.2 Broadband technology roadmap for a 10-year period ........................ 186

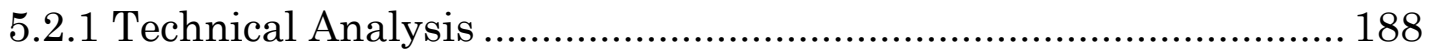

5.2.2 Engineering Costs Analysis......................................................... 190

CHAPTER VI: DISCUSSION AND SUMMARY ….................................... 197

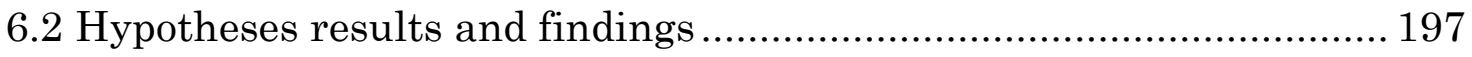

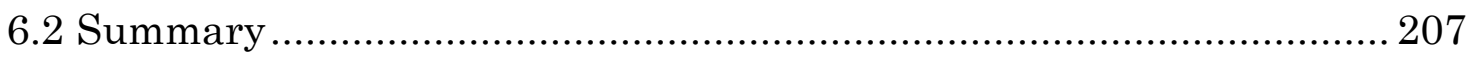

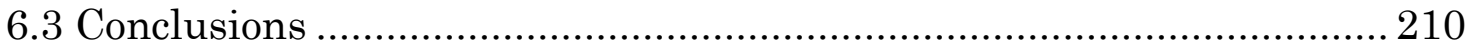

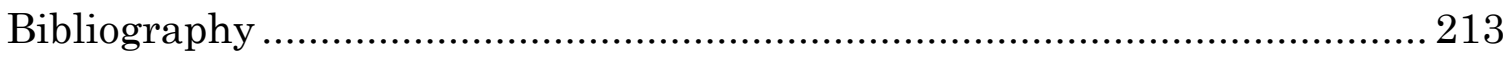

Appendix 1: FITEL Projects and Bids for Broadband Deployments ............ 229

Appendix 2: High Altitude Platforms (HAPs) for Providing Broadband

Services 235

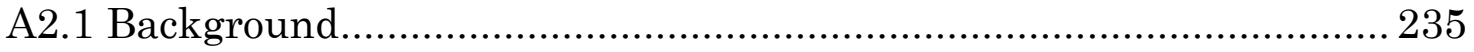

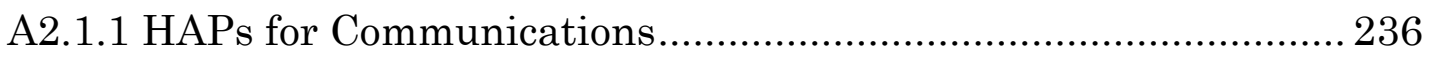


A2.1.2 HAP research and industry initiatives 238

A2.1.3 Telecommunications regulatory and policy environment for HAPs240

A2.2 Stratospheric Environment 241

Stratospheric Winds 242

A2.3 Project Loon (Google X) 245

A2.3.1 Aerial Vehicle and Payload 246

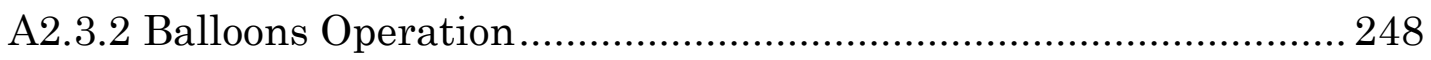

A2.3.3 Balloons Network 250

A2.3.4 Balloon's coverage analysis in the study areas in the Andes and

Amazon regions in Peru. 251

Appendix 3: Calculation of the Effective Download Channel Capacity Calculation 263

A3.1 Maximum Theoretical and Actual Data Rate 263

A3.2 Effective Channel Bandwidth 268

Appendix 4: EDX Simulation Results for Yauli and Samugari. 271 


\section{TABLES}

Table 1. Broadband users by State and Region in 2010 [14].................................. 21

Table 2. Effective download channel and node capacity for Wi-Fi, WiMAX, LTE and

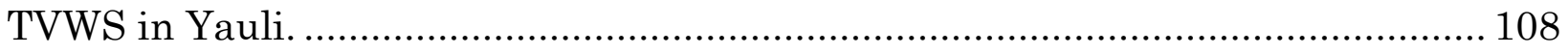

Table 3. Effective download channel and node capacity for Wi-Fi, WiMAX, LTE and

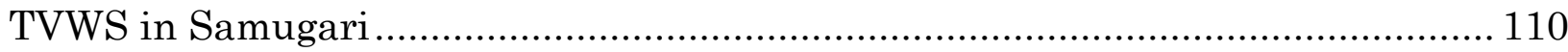

Table 4. WBLAN design results for the Yauli county for the 10-year period for Wi-

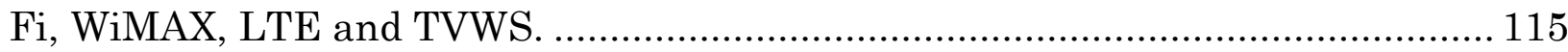

Table 5. WBLAN design results for the Samugari county for the 10-year period for

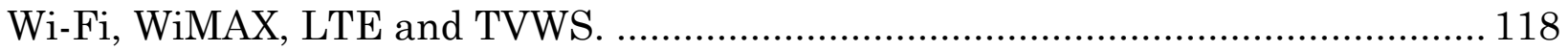

Table 6. WBLAN design results for Yauli for stratospheric balloons using $20 \mathrm{MHz}$

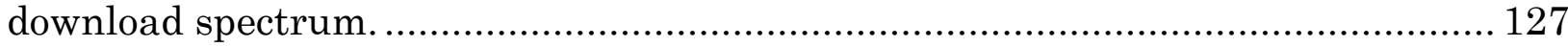

Table 7. WBLAN design results for Samugari for stratospheric balloons using

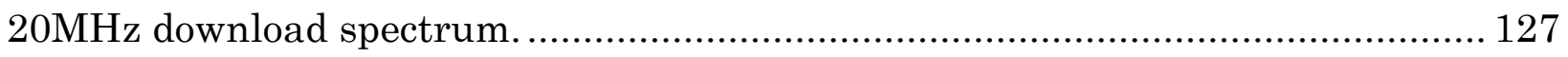

Table 8. NPV percentage variation to a $10 \%$ increase in each CAPEX and OPEX

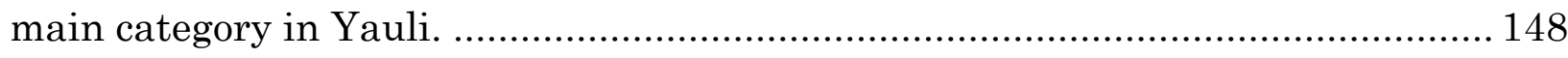

Table 9. Recalculated NPV to a 10\% increase in each CAPEX and OPEX main

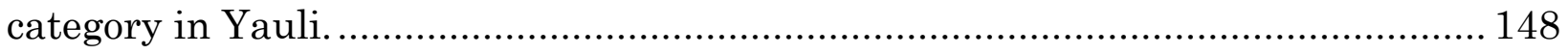

Table 10. NPV percentage variation to a $10 \%$ increase in each CAPEX and OPEX

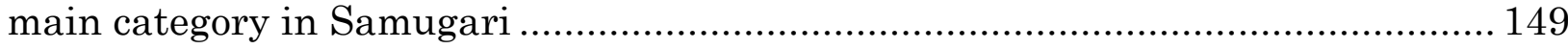

Table 11. Recalculated NPV to a 10\% increase in each CAPEX and OPEX main

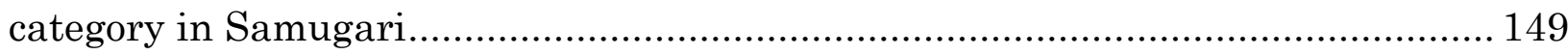


Table 12. Effective download channel and node capacity at different broadband speeds for LTE $40+40 \mathrm{MHz}$ in Yauli

Table 13. Effective download channel and node capacity at different broadband

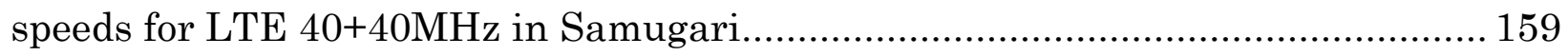

Table 14. WBLAN design results for the Yauli County for LTE 40MHz................ 163

Table 15. WBLAN design results for the Samugari County for LTE 40MHz......... 163

Table 16. FITEL's subsidies for the deployment and operation of transport and access networks in the states of Ayacucho and Huancavelica.

Table 17. Adjusted FITEL's subsidy for the access networks and required initial coverage in the states of Ayacucho and Huancavelica ............................................ 175

Table 18. Access network costs in Yauli (Huancavelica) and initial coverage....... 175

Table 19. Access network costs in Samugari (Ayacucho) and initial coverage ...... 176

Table 20. Cost per user per month in Yauli and in Huancavelica.......................... 178

Table 21. Cost per user per month in Samugari and in Ayacucho ........................ 178

Table 22. Estimated cost of the balloon system including payload, envelope and assembly 262

Table 23. IEEE 802.11n physical layer data rates [134] [135] ...............................264

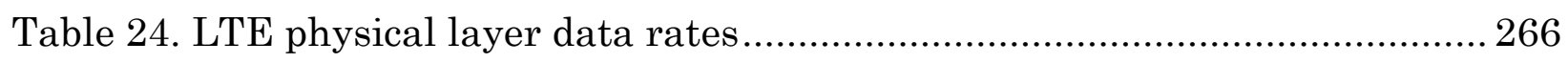

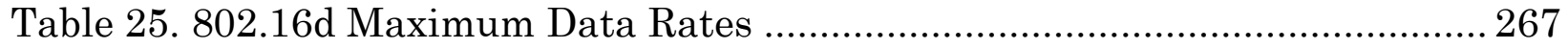

Table 26. Maximum Over the Air (OTA) rates for a commercial broadband system [139] 268 


\section{FIGURES}

Figure 1. Broadband penetration by technology in 2012. Broadband penetration by geographical location in 2012-2013 [16].

Figure 2. Broadband penetration by location and by service type in 2012-2013 [16].20

Figure 3. Distribution of backbone technologies by states. Mobile broadband

coverage. Fixed broadband coverage [14]........................................................... 24

Figure 4. Fiber optic backbone in 2011 and backbone in 2016 [4].........................25

Figure 5. Structure of the technical and engineering cost analysis using a bottom

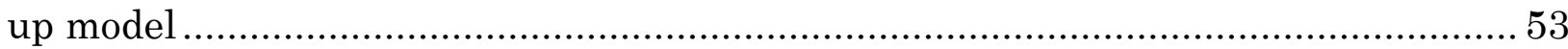

Figure 6. Yauli town, capital of the Yauli county in the state of Huancavelica in the central Andes of Peru. Palmapampa, capital of the Samugari county in the state of Ayacucho in the central Andes and western Amazon of Peru..................................58

Figure 7. Google Earth view of the 27 selected towns in the Yauli county study area

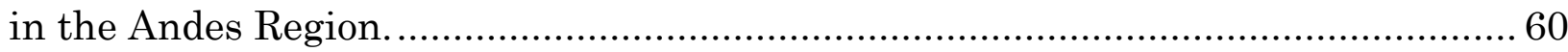

Figure 8. Google earth view of the 29 selected towns in the Samugari county study

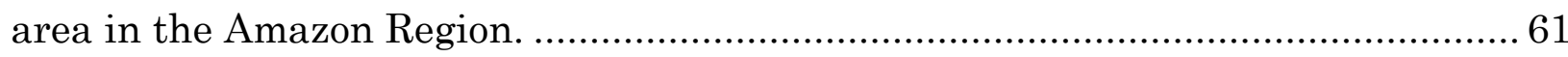

Figure 9. The Internet service price as percentage of monthly income determines

the Internet service penetration or Internet demand curve ..................................... 64

Figure 10. Estimated broadband speed demand 2015-2024 for rural areas in Peru 68

Figure 11. ASTER GDEM V2 terrain data for the Yauli county area in the Andes

Region. Terrain data presented as shaded relief..................................................... 72 
Figure 12. LULC areas. Yauli towns in gray, grassland in orange, and forest in green. Samugari towns in gray, forest in green and river in blue.......................... 74

Figure 13. RF parameter settings in EDX for a base station or access point............ 76

Figure 14. Wireless broadband access network using outdoor Wi-Fi APs for the

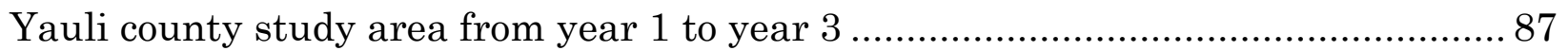

Figure 15. Wireless broadband access network using outdoor Wi-Fi APs for the

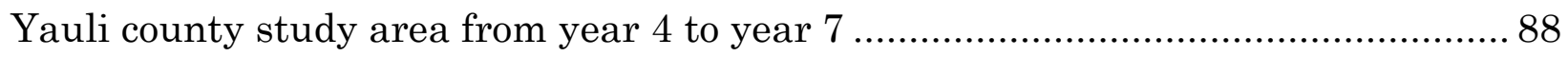

Figure 16. Wireless broadband access network using outdoor Wi-Fi APs for the Yauli county study area from year 8 to year 9 89

Figure 17. Wireless broadband access network using outdoor Wi-Fi APs for the

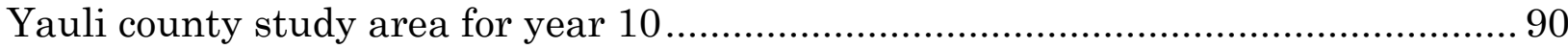

Figure 18. Footprint or coverage of a micro-LTE base station located at 20

kilometers above the ground in the Yauli county study area................................ 91

Figure 19. Modules in Analytica of the engineering cost model for the WBLANs.

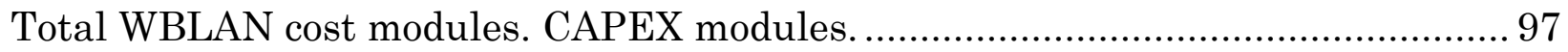

Figure 20. WBLAN using mini-LTE BSs for Yauli from year 1 to year 3............... 105

Figure 21. Users and Mbps capacity per node and users and Mbps per $\mathrm{MHz}$ for current wireless technology options in Yauli

Figure 22. Users and Mbps capacity per node and users and Mbps per $\mathrm{MHz}$ for current wireless technology options in Samugari. 
Figure 23. Coverage of a micro-LTE BS carried in a stratospheric balloon above the Yauli area. Towns are shown in blue. High, mid, and low data rate areas are shown in orange, yellow and green. 121

Figure 24. Coverage of a micro-LTE BS carried in a stratospheric balloon above the Samugari area. Towns are shown in blue. High, mid, and low data rate areas are shown in orange, yellow and green.

Figure 25. Fixed broadband demand and micro-LTE BS capacity for $20+20 \mathrm{MHz}$ spectrum in Yauli using one balloon.

Figure 26. Fixed broadband demand and micro-LTE BS capacity for $20+20 \mathrm{MHz}$ spectrum in Samugari using two balloons.

Figure 27. Total cumulative CAPEX and OPEX for the deployment and operation of the WBLANs for the 10-year period in Yauli. 130 Figure 28. Total cumulative CAPEX and OPEX for the deployment and operation of the WBLANs for the 10-year period in Samugari. 131

Figure 29. CAPEX and OPEX NPV of WBLANs in Yauli and Samugari.

Figure 30. Yauli's CAPEX NPV breakdown in the main categories: energy system,

civil engineering, towers, $\mathrm{CPE}$ and network equipment.

Figure 31. Samugari's CAPEX NPV breakdown in the main categories: energy system, civil engineering, towers, CPE and network equipment.

Figure 32. Yauli's OPEX NPV breakdown in the main categories: HQ operation, network electricity, personnel, operation fees and F.O. interconnection. 
Figure 33. Samugari's OPEX NPV breakdown in the main categories: HQ operation, network electricity, personnel, operation fees and F.O. interconnection.139

Figure 34. Cost per user per month for the Yauli County study area..................... 142

Figure 35. Cost per user per month for the Samugari County study area ............. 143

Figure 36. Cost per Mbps per month for the Yauli County study area ................... 145

Figure 37. Cost per Mbps per month for the Samugari County study area ........... 146

Figure 38. NPV increase for AP/BS cost variation in Yauli................................... 151

Figure 39. NPV increase for AP/BS cost variation in Samugari ............................ 152

Figure 40. Self-supported tower (Left) and cable supported tower (Right)............. 153

Figure 41. Self-supported and cable-supported towers NPV in Yauli.................... 154

Figure 42. Self-supported and cable-supported towers NPV in Samugari ............. 155

Figure 43. Capacity for wireless technologies in Yauli including LTE 40MHz...... 161

Figure 44. Capacity for wireless technologies in Samugari including LTE 40MHz.161

Figure 45. Total cumulative CAPEX and OPEX for a 10-year period for LTE

40MHz and Wi-Fi, WiMAX, LTE (20MHz) and TVWS in Yauli........................... 164

Figure 46. Total cumulative CAPEX and OPEX for a 10-year period for LTE

40MHz and Wi-Fi, WiMAX, LTE $(20 \mathrm{MHz})$ and TVWS in Samugari ...................... 165

Figure 47. NPV of LTE 40MHz and current wireless options in Yauli.................. 166

Figure 48. NPV of LTE 40MHz and current wireless options in Samugari ........... 166

Figure 49. Cost per user per month for the Yauli County including LTE 40+40MHz168

Figure 50. Cost per user per month for the Samugari County including LTE

$40+40 \mathrm{MHz}$ 169 
Figure 51. Cost per Mbps per month for the Yauli County including LTE $40+40 \mathrm{MHz}$. 170

Figure 52. Cost per Mbps per month for the Samugari County including LTE $40+40 \mathrm{MHz}$ 171

Figure 53. NPV of LTE $20+20 \mathrm{MHz}$ in Yauli at different oversubscription rates:

$10: 1,5: 1$, and $20: 1$. 181

Figure 54. NPV of LTE $20+20 \mathrm{MHz}$ in Samugari at different oversubscription rates:

10:1, 5:1, and 20:1.

Figure 55. Total cumulative CAPEX and OPEX for the deployment and operation of stratospheric balloons in the Yauli area using overbooking ratios of 10:1, 5:1 and

$20: 1$ 183

Figure 56. Total cumulative CAPEX and OPEX for the deployment and operation of stratospheric balloons in the Samugari area using overbooking ratios of 10:1, 5:1

and 20:1. 184

Figure 57. Fixed broadband demand and micro-LTE BS capacity for $20+20 \mathrm{MHz}$ and $40+40 \mathrm{MHz}$ spectrum in Yauli (one balloon) and Samugari (two balloons)...... 189 Figure 58. Combined (Yauli and Samugari) NPV of potential roadmap technology options

Figure 59. Cumulative CAPEX \& OPEX of the combined (Yauli and Samugari)

deployment and operation costs of roadmap technology options 193

Figure 60. Annual averaged wind speeds at different latitudes from 1948 to 2002 at $20 \mathrm{~km}$ above the ground [127]. 242 
Figure 61. Wind speed profiles from August 13 with Helios TAS limit and PMRF August wind climatology. [128] 243

Figure 62. Wind direction profiles from August 13 with PMRF August wind direction climatology [127]. 244

Figure 63. Super-pressure balloons altitude path at day and night [130] ..............247

Figure 64. Estimation of wind speed and direction between 50kft and 60kft. .......254

Figure 65. Balloon flight route over the Yauli study area.................................... 255

Figure 66. Balloon flight route over the Samugari study area ............................. 256

Figure 67. Balloon flight route test in South America .......................................... 258

Figure 68. Estimation of wind speed and direction between $60 \mathrm{kft}$ and $80 \mathrm{kft}$........ 259

Figure 69. Balloon flight route over the Yauli study area for the case of a route in

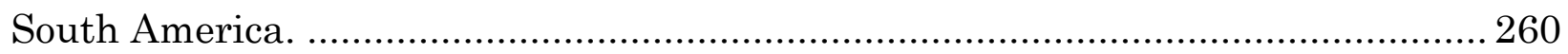

Figure 70. WBLAN using mini-LTE BSs for Yauli from year 1 to year $3 \ldots \ldots \ldots \ldots \ldots . . .271$

Figure 71. WBLAN using mini-LTE BSs for Yauli from year 4 to year 7 .............. 272

Figure 72. WBLAN using mini-LTE BSs for Yauli from year 8 to year 9.............. 273

Figure 73. WBLAN using mini-LTE BSs for Yauli for year 10............................2 274

Figure 74. WBLAN using mini-LTE BSs for Samugari from year 1 to year 7 ......2275

Figure 75. WBLAN using mini-LTE BSs for Samugari from year 8 to year 9. ..... 276

Figure 76. WBLAN using mini-LTE BSs for Samugari in year 10 ...................... 277 


\section{CHAPTER I}

\section{INTRODUCTION}

\subsection{Statement of the problem and research question}

For several decades, the landscape in South America (the Andes range and the Amazon Rainforest) has posed one of the main barriers to carrying out large scale infrastructure deployments (i.e., road, power grid, or telecommunication deployments) in the rural areas. As a result, in Peru, economic, education and healthcare indicators show high levels of poverty, school absenteeism and a critical situation in children mortality rate, maternal health and malaria incidence in the country's rural areas [1] [2]. Furthermore, the global trend of the digital and Internet era has increased the gap between urban and rural areas. In the last 5 years, several governments in South America, recognizing the importance of broadband for economic and social development, have been proactive to design and implement national broadband plans [3]. Leading plans in Latin America include: National Telecommunications Plan Connected Argentina (Argentina), National Plan of Large Band (Brazil), All Chile Connected Plan (Chile), Live Digital Plan (Colombia), Digital Agenda.mx (Mexico) and National Plan for Development of Broadband in Peru (Peru). In order to increase the penetration of broadband in 
their countries, most of these plans have a similar first stage component of deploying or expanding the national fiber-optic backbone network to interconnect main urban areas and reach rural areas. Currently, these governments are focusing efforts and resources on designing the national backbones, setting bids for construction, and developing the appropriate regulatory framework for use of the backbone network. For example, Peru is now building a new 13,000 Km national fiber backbone that will expand backbone coverage to 180 provinces within 22 states of the country including urban and rural areas [4]. The aim is to increase the $7.52 \%$ broadband penetration reported in 2012 [5].

Once this backbone is deployed, the next big challenge that governments and broadband service providers will face is the deployment and operation of local access networks in unserved and underserved peri-urban and rural areas, and to reach residents where broadband legacy networks are minimal or non-existent today. Telecom service providers in rural areas will have to operate under difficult circumstances, including: 1) high-cost conditions due to a rough, undeveloped landscape, 2) predominately low income households, and 3) large geographic regions with a very low density of customers [6]. In this scenario, there is a high level of uncertainty as to the best local access technology to deploy and operate. In short, governments are struggling to develop and identify the technology roadmaps for broadband services in less-developed peri-urban and rural settings.

The study "Technologies and Policies to Connect the Next 5 Billion" [7] attempts to provide solutions for this question by describing a broadband 
deployment framework identifying the most promising technologies to connect underserved areas in emerging countries to the Internet. This study describes how to develop broadband roadmaps, representing the best local access network technology fit, considering technology alternatives which have the potential to provide the most economical combination of network coverage and broadband speed for a given population density in underserved areas. While [7] provides a qualitative framework for developing a broadband roadmap for underserved areas, beyond concluding that early solutions will be wireless, it does not provide any concrete recommendations for the best technical wireless options to deploy over time. Such recommendations require application of a detailed engineering cost analysis that can compare the costs of different local access network technologies over a specific time period. This research attempts to take this next step and to address the research question: What is the roadmap for introducing broadband services to unserved and underserved areas in the Andes and Amazon Regions of Peru? We develop a more definitive broadband roadmap for the Andes and Amazon regions of Peru based upon a rigorous, quantitative analysis of the costs and capabilities of practical wireless options. This effort breaks down into three research subproblems which answer the following questions:

1. What are the current leading wireless technology options to consider for providing broadband services in underserved and unserved areas of developing countries, and how can they be designed for deployment in this setting? This question will lead to evaluate current and new disruptive 
wireless technologies and platforms to provide broadband services in rural areas in developing countries. Additionally, we will evaluate appropriate technical design and configuration settings for these technologies and platforms by conducting propagation modelling and simulating wireless access networks.

2. How do the deployment and operating cost for this set of wireless technology options compare with each other, and what is the optimal roadmap for deployment of these technologies over the next 10 years? We will develop a detailed engineering cost model and conduct a cost analysis to estimate deployment and operating costs of each technology for a 10-year period and determine the most cost/effective technology, or set of technologies, to serve rural areas. This model will include specific cost elements for each technology.

3. What are the policy implications as a result of this detailed engineering economic analysis of wireless broadband technology options? We will conduct an engineering cost analysis to different spectrum policy and access network requirement scenarios, and conduct also a sensitivity analysis to determine the implications and cost impact of current and new proposed policies.

\subsection{Research hypotheses}

We address these subproblems by testing the following hypotheses: 
Hypothesis 1: Deploying wireless access networks in the Andes and Amazon regions of Peru using the Wi-Fi technology and unlicensed spectrum has the lowest deployment and operating cost in a 10-year period. We will test this hypothesis by developing an engineering cost model to estimate the cost of deploying and operating terrestrial wireless access networks employing current wireless technologies (i.e. Wi-Fi, WiMAX, LTE and TV White Spaces) and new stratospheric wireless access networks (i.e. using stratospheric balloons and unmanned aerial vehicles) employing a subset of the wireless technologies.

Hypothesis 2: Wireless access networks using stratospheric platforms (i.e. stratospheric balloons and unmanned aerial vehicles) in the Andes and Amazon regions of Peru has the lowest deployment and operating cost for the initial years but are not able to scale for a 10-year period to serve forecasted customer and speed growth. We will test this hypothesis by using our engineering cost model to estimate the cost of providing broadband services using stratospheric platforms, and also comparing the broadband capacity provided by these platforms against the estimated customer and speed demand growth in a 10-year period.

Hypothesis 3: Spectrum management and regulation have a quantifiable technology and economic impact in the deployment and operation of wireless access networks in the Andes and Amazon regions of Peru and are important to define the best technology fit to introduce broadband services in these regions. We will test this hypothesis by proposing spectrum management and regulation scenarios for the spectrum used by the different technology options and, using our engineering cost 
model, evaluating their impact on the total deployment and operating cost over a 10-year period.

\subsection{Delimitation of the Study}

The delimitations and scope of interest of this study include the following:

- The study focusses on unserved and underserved peri-urban and rural areas in the Andes and Amazon regions of Peru with emphasis on areas targeted by the National Plan for Development of Broadband in Peru. This plan aims to provide broadband services to counties in 180 provinces in 22 states in the next 10 years. For the analysis, two counties were selected as representative scenarios of peri-urban and rural areas in the Andes and Amazon regions. The two counties were selected due to, first, their low broadband penetration in peri-urban and rural areas, second, rural remote locations, and, third, harsh landscape settings which will help to analyze wireless technologies performance in extreme conditions.

- In the two chosen counties, the geographical service coverage was defined based on the following criteria: 1) minimum service coverage defined by the National Plan for Development of Broadband in Peru, and, 2) additional service coverage based on reaching other potential markets and estimating organic growth of the broadband network and service in a 10-year period. These criteria is a combination of the minimum service coverage (universal service mandatory market) in the towns defined in the National Broadband 
Plan with additional coverage based on potential broadband service demand of neighboring towns (potential additional markets).

- The technical analysis of terrestrial wireless broadband access networks includes the following technologies: Wi-Fi, WiMAX, LTE and TV White Spaces. These technology options were chosen due to their current market adoption by wireless broadband service providers in several countries around the world (i.e. Wi-Fi, WiMAX and LTE) or their expected potential for increasing the capacity of backbone and coverage (i.e. TV White Spaces) of wireless access networks. These technologies are approved or likely to be approved by Peruvian telecommunication regulation.

- A subset of these wireless technologies is chosen for a technical analysis of stratospheric wireless access networks using unmanned aerial systems (stratospheric balloons and aircrafts). Stratospheric access networks were included in the technical analysis due to current innovative and disruptive research initiatives led by Google and Facebook which aim to provide wireless broadband service to rural remote areas in developing countries using unmanned aerial systems.

- The wireless access network design and propagation modeling used the EDX Signal Pro software, which allows using different propagation models such as the free space, empirical, stochastic, and physical models. EDX also allows using digital terrain data, land use and land coverage (LULC) data and digital satellite images to identify different land use and coverage areas. 
- The engineering cost analysis used the Analytica software, a specialized spreadsheet software package appropriate for the development of complex economic models with the availability to conduct parametric uncertainty analysis to different outcomes of the model. Conducting this analysis included generating an engineering cost model, which in this case used a bottom-up approach. This model allowed estimating the deployment and operation cost of efficient state-of-the-art wireless broadband access networks. The technical design results provided inputs for the engineering cost model. The main cost categories in the engineering cost model for the deployment and operation of the access networks for a 10-year period are the following: 1) Capital Expenditures (CAPEX) (i.e. tower, civil engineering, energy systems, network equipment and customer premise equipment) and 2) Operating Expenditures (OPEX) (i.e. operation, preventive maintenance, and reactive maintenance). The detailed elements in each cost category is based on reported network design and operation best practices defined by broadband service providers, network deployment and operation contractors, equipment vendors, and radio frequency $(\mathrm{RF})$ engineers and technicians expert on network deployments.

- The results of the technical and engineering cost analyses allowed, first, to quantify and compare the technical performance and deployment and operation cost of the wireless technology options, and second, to develop the technology roadmap to introduce broadband services in the Andes and 
Amazon regions of Peru. For achieving this two-fold purpose, we used the following cost metrics: 1) Net present value (NPV), 2) cost per Mbps per month, and 3) cost per user per month.

- Developing a technology roadmap to introduce broadband services in periurban and rural areas in the Andes and Amazon regions of Peru also required analyzing new spectrum policies and regulation scenarios which impact the technical design and deployment and operations cost of the wireless access networks. The policy and regulation analysis in this study proposes and analyzes the technical and cost impact of spectrum management recommendations for the wireless technology options to determine best technical and economic solution in a 10-year period.

\subsection{Limitations of the Study}

The limitations and restrictions of this research include the following:

- The network design and propagation analysis used technical specifications of the RF equipment obtained from datasheets and manuals available on vendor websites or made available by vendor representatives. In actual wireless access network deployments, it is recommended to conduct actual measurements to validate $R F$ specifications of the equipment in order to factor for excess or defect specifications values in the network design (i.e., transmission power, antenna gain, maximum client load, etc.). A limitation in the network design is not being able to use technical specifications 
obtained from actual RF equipment measurements. However, in order to reduce the impact of this limitation, the network design and propagation analysis includes an excess signal margin which allows providing broadband service in the event of facing unexpected losses or any equipment performing below datasheet specifications.

- Because there are neither currently commercially available unmanned aerial systems (stratospheric balloons or aircrafts) for stratospheric access networks nor have been deployed in commercial broadband service networks, a limitation for this study is that there are neither reported technical specifications nor cost data of these systems. To address this limitation, we use up-to-date technical descriptions of the components of the system (released by Google and Facebook) to forecast technical performance and estimate the cost of the system based on the cost of commercially available components of the system.

- The wireless access network design using different technology options is based on EDX software simulations. Field trips were not conducted in neither of the two study areas nor consultations with network contractors and engineers who have conducted deployments in these rural areas. A limitation in this study is not being able to corroborate the terrain and land use in the two study areas. The impact of this limitation was reduced by using up to date digital 30meter-resolution terrain data from NASA and 
digital satellite images from Google and Bing to simulate accurately the two study areas.

- Cost data for RF and other equipment were obtained in different types (quotations, documents, invoices, reports, interviews, etc.), from different countries (Peru, United States, China, Germany, etc.) and from different sources (Investment Fund in Telecommunications (FITEL), RF equipment vendors, access network deployment contractors, among others). To request the cost data, this research topic was introduced and explained to the different cost data sources. Since it was clear to many sources that our inquiry would not end up in an actual equipment purchase order in the short term, a limitation in the study is not assuring that the cost data is the best price an actual broadband service provider would obtain. To reduce the impact of this limitation, for each type of equipment we collected cost data from several sources and verified that comparable competing equipment have prices within the same range.

The study assumptions include the following:

- The broadband customer and speed demand, and their growth over a 10-year period, in the two underserved and unserved study areas can be estimated based on population and broadband penetration statistics from currently served areas. This study used general population statistics (i.e. population, households, income, etc.) at state and county levels from the Peruvian National Institute of Statistics and Information (INEI), and Internet access 
and broadband service statistics (i.e. historical residential penetration, broadband speed penetration, Internet access rates, etc.) at state level from the Supervisory Agency of Private Investment in Telecommunications (regulatory agency), the Telecommunications Investment Fund (universal service fund agency), and the Ministry of Transport and Communications.

- The service coverage analysis estimates populated areas based on satellite images showing location of the household clusters. However, neither satellite images nor maps provide accurate information of the total areas of towns and borders between them. The populated areas were defined based on identifiable household clusters in the satellite images, and assumed a uniform household distribution in each town.

- The land used to place nodes (including tower, infrastructure, and RF and associated equipment) in the network design can be purchased for the access network.

- The cost provided by different sources is accurate and truthful. It was also assumed that cost data from equipment sold in different countries can be compared against equipment sold in Peru by adding shipping, import tax and other associated fees.

\subsection{Importance of the Study}

Several countries and organizations in the world have recognized the importance of broadband for economic and social development, as the following: 
- According to the World Bank, broadband contributes to increased productivity and economic growth, and therefore, countries should assign broadband an important role in national development strategies [8].

- An increase in $10 \%$ of broadband connections in a country results in an economic growth increase of $1.3 \%$ [9].

- A $10 \%$ increase in broadband penetration in a specific year correlates with a work productivity increase of $1.5 \%$ in the subsequent five years [10].

- In the Sao Paulo declaration at the 2010 First Ibero-American Forum for Broadband Development [11], countries recognized: 1) the importance of broadband as essential infrastructure for social and economic development, and 2) its importance in decreasing economic, regional and social inequalities and in providing access to information and knowledge democratization.

In this context, several developed and developing countries have designed national broadband plans to increase broadband penetration in the short and long run. In developing countries, the most challenging task will come from attempting to provide affordable broadband services to underserved and unserved peri-urban and rural areas. This will be challenging due to the lack of legacy infrastructure, high deployment cost, and difficult market conditions, among other entry barriers.

In these conditions, identifying the best broadband technologies to deploy services in unserved and underserved areas is a very difficult problem to solve. This research aims to provide some well-reasoned advice on the most attractive 
wireless technologies for the Andes and Amazon regions of Peru. In fact, the research problem for this study comes directly from FITEL (universal service agency in Peru) as a key problem currently facing the Peruvian government in planning for broadband penetration expansion. As a result, this research will generate a refined broadband technology roadmap for the next 10 years for these regions. We will generate the roadmap by developing and using an engineering cost model tailored for scenarios in the Andes and Amazon regions.

The results and findings of this research (the engineering cost model and the broadband technology roadmap) will be made available to government agencies and regulators in Peru, South America, and other developing countries as a source of critical information to assist in the planning for the deployment of local access networks as part of their national broadband plans. The Peruvian central government, as well as state and local governments, have expressed their need and interest for such research results. The results will also be made available to the following: 1) incumbent and new broadband service providers, entrepreneurs and local organizations which would require assessment for carrying out broadband deployments in rural areas and generating new and innovative business models, 2) content, service, and application providers in Peru and the world which seek to reach emerging markets and new customers in developing countries to generate new and innovative business models. 


\section{CHAPTER II}

\section{REVIEW OF THE LITERATURE}

\subsection{Broadband Landscape in Latin America and Peru}

The population of Peru is 31.3 million (reported in 2015). In the last decade, Peru presented a positive economic environment [12]: 1) sustained annual average economic growth of $6.2 \%$ (2003-2010), 2) average inflation of 3\% (2004-2010), 3) responsible and sound fiscal policy, 4) continuous improvement in country risk qualifications, 5) good stock index performance, and 6) active role of public investment, through infrastructure deployment, etc. This positive economic growth was the result of global increasing demand for commodities in Latin America (mining, oil, gas and agricultural products) [3] and for exports from the industrialized coastal region of Peru (oil refining, furniture, textiles, clothing, fishing and fish processing, food processing, etc.). However, this economic growth was mainly focused on urban centers in the coastal region and was not uniform among the other two main regions, the Andes and Amazon regions. Therefore, to expand the economic growth to the Andes and Amazon regions, in both urban and rural areas, in 2011, the Peruvian central government designed national development plans targeting commerce, agriculture, education, healthcare, 
governance, and telecommunications, among others areas. This study focuses on the national telecommunications plan for broadband development launched in 2011.

In the last five years, Peru and other countries in Latin America, recognizing the importance of broadband for economic and social development, have been proactive to design and begin execution of national broadband plans. The current most important plans in Latin America, and broadband penetration reported in 2011, are the following [13]: National Telecommunications Plan Connected Argentina (11.63\% of population and $39.32 \%$ of households), National Plan of Broadband in Brazil (8.8\% of population and 29.1\% of households), All Chile Connected Plan (11.63\% of population and $39.32 \%$ of households), Live Digital Plan in Colombia (7.28\% of population and $25.5 \%$ of households), Digital Agenda.mx in Mexico (11.02\% of population and 41\% of households) and National Plan for Development of Broadband in Peru (4.2\% of population and 17\% of households). Most of these plans include public-private cooperation components which will vary depending on the levels and conditions of government funding in each country. For example, Peru's model has created a fund to be granted in open bids to private operators for development of broadband; Chile follows a traditional model which offers subsidies to private operators, also granted in open bids; in the model of Brazil and Argentina, the government-owned operator funds and deploys the backbone of the nationwide network which enables private operators to provide service to final customers [13]. 
In this regional context, most of these countries are focusing on expanding affordable broadband service especially in underserved and unserved areas. The subsection below presents broadband penetration in these areas in Peru.

\subsubsection{Broadband penetration in the Andes and Amazon regions in Peru}

The definition of broadband service is not uniform and varies depending on the standard organization or country. For example, the International Telecommunications Union (ITU) defines broadband service as data transmission services with download rates equal or higher to $256 \mathrm{kbps}$. In Peru, broadband service was defined as data transmission services with rates higher than dial-up (56kbps) [14]. Based on this definition, the broadband penetration in Peru was 7\% of the population in 2012, a slight increase from $3.46 \%$ in 2010 and $4.2 \%$ in 2011 [15]. Both fixed and mobile broadband services expansion in recent years in major urban areas have contributed towards higher broadband penetration. In March 2011, the fixed broadband market share was $76 \%$ (991,089 subscribers) of the total broadband market, and the mobile broadband market share was $24 \%(312,976$ subscribers). By the end of 2012, the fixed broadband market share was $69 \%$ (1,563,217 subscribers) and the mobile broadband market share was $31 \%(702,315$ subscribers) [15]. The fixed broadband service is provided using wired and wireless technologies such as ADSL, cable, WiMAX, VSAT and dedicated lines (fiber optic). Mobile broadband service is provided using $3 \mathrm{G}$ and recently $4 \mathrm{G}$ LTE devices. Figure 1 shows the market share for different broadband technologies in 2012. We can 
observe that almost $60 \%$ of the total broadband market uses ADSL, which is also $86 \%$ of the fixed broadband market. This indicates most of the broadband service is provided to final customers using legacy telephone networks, which were mainly deployed in urban areas.

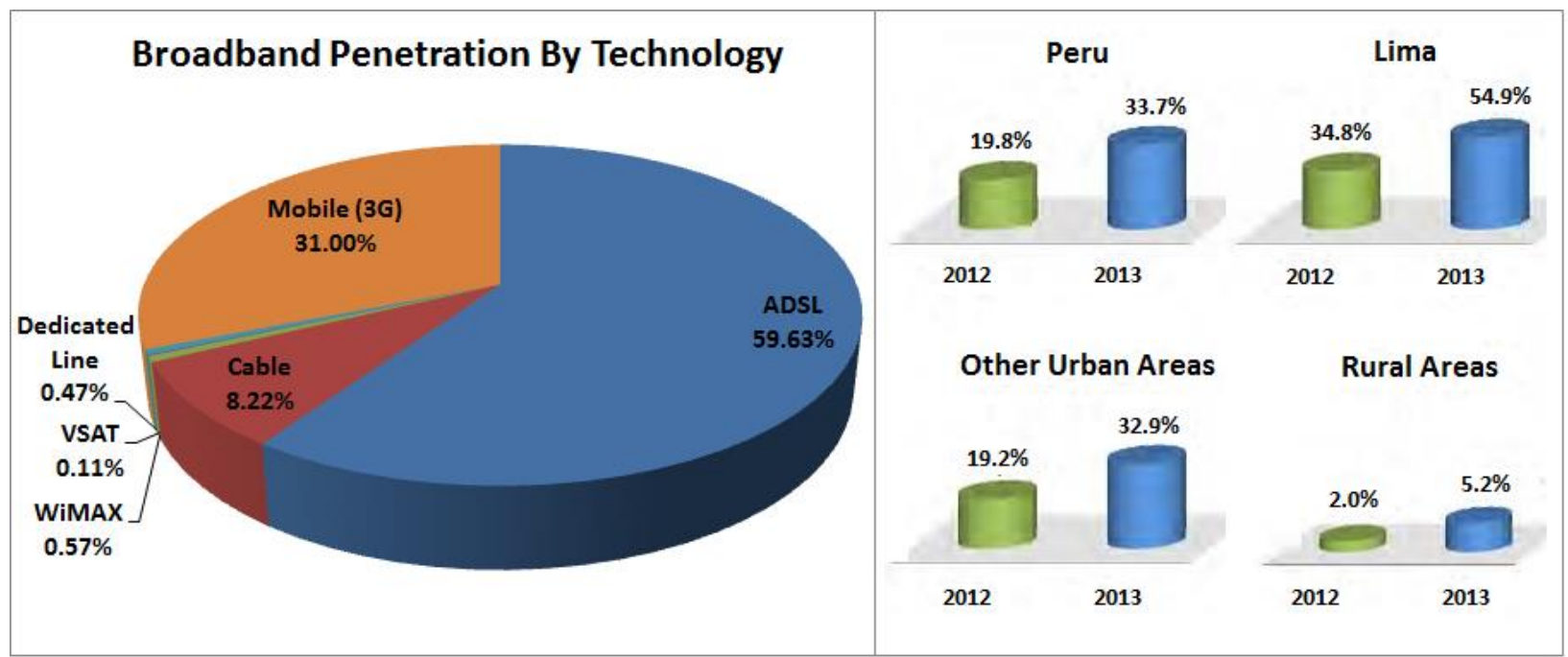

Figure 1. (Left) Broadband penetration by technology in 2012. (Right) Broadband penetration by geographical location in 2012-2013 [16].

In addition to broadband penetration data, in this study we also use Internet penetration as a proxy for broadband penetration. After 2004, the number of narrowband users (mostly using dial-up) started to decrease while the number of broadband users increased, and in 2009 and 2012 narrowband users were $2.17 \%$ and $0.3 \%$ of all Internet users, respectively [15]. According to a 2012-2013 residential Internet service survey conducted by the telecom regulator OSIPTEL [16], Figure 1 shows that in 2013 Peru had a residential penetration of $33.7 \%$ of households. Breaking down this nationwide penetration, the country's capital (Lima) and other urban areas were the main contributors towards this penetration; 
Lima, which has around one third of Peru's households, had 54.9\% Internet residential penetration, and other urban areas had a penetration closer to the national penetration, $32.9 \%$. On the other hand, rural areas had the lowest Internet penetration of $5.2 \%$. This penetration level confirms that most unserved and underserved areas are located in rural areas. From the same OSIPTEL survey, Figure 2 breaks down Internet service penetration based on mobile and fixed services, which shows that the service is provided by cell phone networks and other types of networks (legacy PSTN, cable, satellite and other wireless), respectively. Figure 2 shows that in 2013 in rural areas $3.7 \%$ of households used only mobile broadband, $1.2 \%$ used only fixed broadband and $0.3 \%$ used mobile and fixed broadband. The greater (three times) adoption of mobile over fixed broadband services in rural areas is due to the lack of or limited deployment of legacy networks (ADSL and cable) in rural areas.

The broadband penetration in Peru can also be presented according to geographical location considering the 24 states of Peru grouped in 3 main regions: the Coastal, Andes and Amazon regions. In many cases, states are located in two regions. For example, Cusco occupies geographically both the Andes and Amazon regions; however, since most of its population lives in the Andes region, it is included in this region. Table 1 shows the broadband penetration by state and region in 2010 [14]. We can observe that $90.68 \%$ of the broadband users are located in the Coastal region, while only the remaining $7.79 \%$ and $1.5 \%$ are located in the Andes and Amazon regions. We can also observe that in the Andes and Amazon 
regions, the penetration in each state is equal or lower than $2 \%$, and in most cases even lower than $1 \%$.

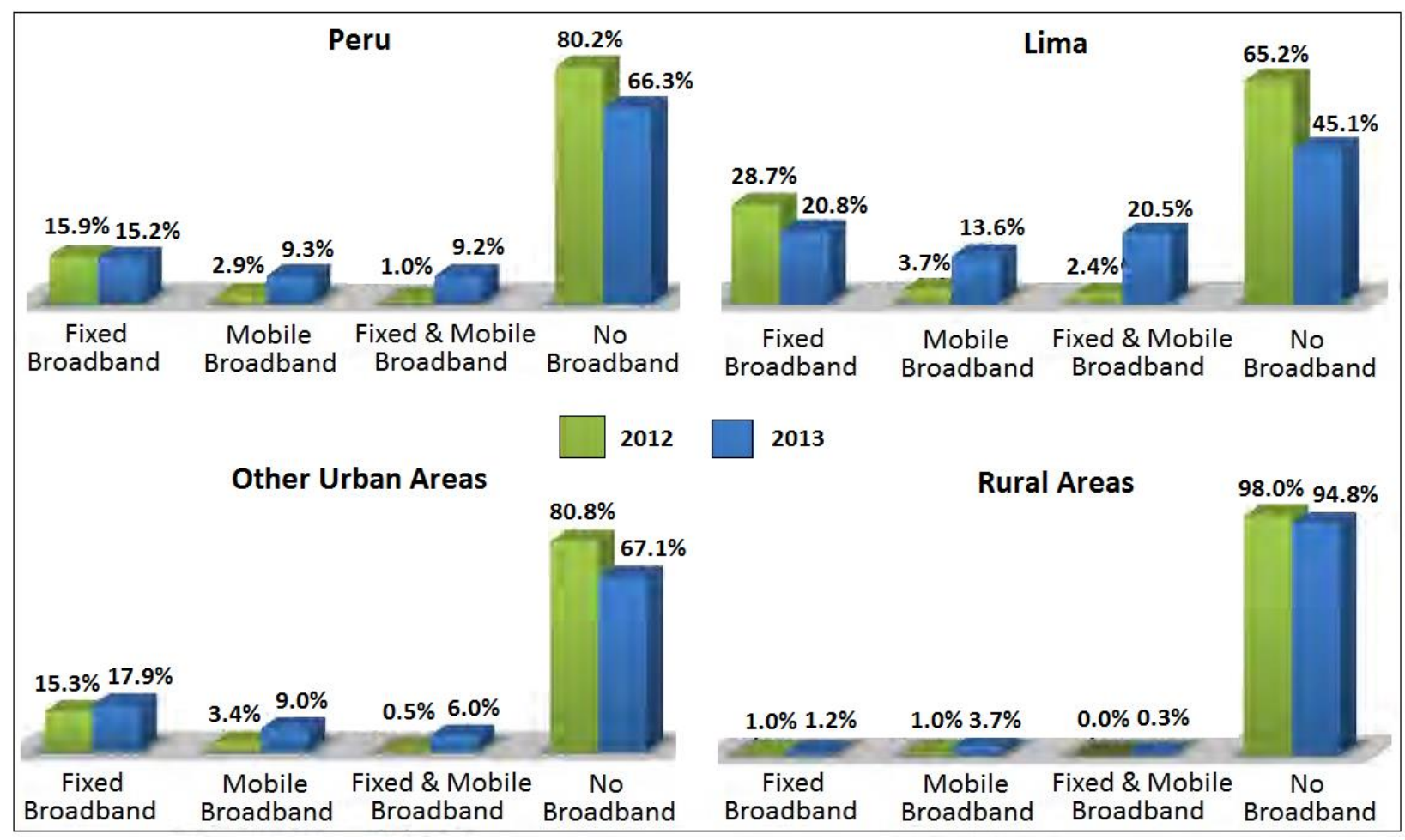

Figure 2. Broadband penetration by location and by service type in 2012-2013 [16].

Thus, broadband penetration data indicates that areas with the lowest broadband penetration, or most unserved and underserved in Peru, consist of the following: 1) rural areas, and 2) states in the Andes and Amazon regions. Accordingly, this study choses and analyzes these two study areas. 
Table 1. Broadband users by State and Region in 2010 [14].

\begin{tabular}{|c|c|c|c|c|c|}
\hline & \multirow[b]{2}{*}{ State } & \multicolumn{4}{|c|}{ Broadband } \\
\hline & & Users & $\begin{array}{c}\text { Market } \\
\text { Share (\%) }\end{array}$ & Penetration & $\begin{array}{c}\text { Combined } \\
\text { Market Share } \\
(\%)\end{array}$ \\
\hline \multirow{10}{*}{$\begin{array}{l}\text { Coastal } \\
\text { Region }\end{array}$} & Lima y Callao & 889,974 & $68.25 \%$ & $8.75 \%$ & \multirow{10}{*}{$90.68 \%$} \\
\hline & La Libertad & 64,152 & $4.92 \%$ & $3.64 \%$ & \\
\hline & Arequipa & 63,024 & $4.83 \%$ & $5.13 \%$ & \\
\hline & Piura & 40,219 & $3.08 \%$ & $2.26 \%$ & \\
\hline & Lambayeque & 38,538 & $2.96 \%$ & $3.17 \%$ & \\
\hline & Ancash & 30,577 & $2.34 \%$ & $2.73 \%$ & \\
\hline & Ica & 27,562 & $2.11 \%$ & $3.66 \%$ & \\
\hline & Tacna & 16,156 & $1.24 \%$ & $5 \%$ & \\
\hline & Moquegua & 6,699 & $0.51 \%$ & $3.88 \%$ & \\
\hline & Tumbes & 5,721 & $0.44 \%$ & $2.55 \%$ & \\
\hline \multirow{9}{*}{$\begin{array}{l}\text { Andes } \\
\text { Region }\end{array}$} & Cajamarca & 15,733 & $1.21 \%$ & $1.04 \%$ & \multirow{9}{*}{$7.79 \%$} \\
\hline & Huánuco & 7,924 & $0.61 \%$ & $0.95 \%$ & \\
\hline & Pasco & 1,716 & $0.13 \%$ & $0.58 \%$ & \\
\hline & Junín & 24,318 & $1.86 \%$ & $1.86 \%$ & \\
\hline & Huancavelica & 1,550 & $0.12 \%$ & $0.32 \%$ & \\
\hline & Ayacucho & 6,950 & $0.53 \%$ & $1.06 \%$ & \\
\hline & Apurimac & 3,046 & $0.23 \%$ & $0.68 \%$ & \\
\hline & Cusco & 25,867 & $1.98 \%$ & $2.02 \%$ & \\
\hline & Puno & 14,609 & $1.12 \%$ & $1.07 \%$ & \\
\hline \multirow{5}{*}{$\begin{array}{l}\text { Amazon } \\
\text { Region }\end{array}$} & $\begin{array}{c}\text { Madre de } \\
\text { Dios }\end{array}$ & 1,963 & $0.15 \%$ & $1.59 \%$ & \multirow{5}{*}{$1.50 \%$} \\
\hline & Ucayali & 6,394 & $0.49 \%$ & $1.36 \%$ & \\
\hline & San Martín & 7,336 & $0.56 \%$ & $0.93 \%$ & \\
\hline & Amazonas & 1499 & $0.11 \%$ & $0.36 \%$ & \\
\hline & Loreto & 2,538 & $0.19 \%$ & $0.26 \%$ & \\
\hline
\end{tabular}




\subsubsection{National broadband plan: backbone and access networks}

This section presents the two major telecommunications infrastructure and network deployment components led by the Peruvian government (including executive, regulatory and universal service agencies) under the framework of the national broadband plan [14]. More details on the objectives of the national broadband plan and government led regulation and policies for nationwide broadband development are provided in Section 2.4 Regulatory and Policy Environment for Providing Broadband Services in Peru. These infrastructure and network deployment components are the roll-out of, first, a nationwide fiber optic backbone, and second, local access networks. Both roll-outs are funded or subsidized by the government under the public-private-partnership (PPP) model. The following describes recent progress deploying both network components.

\section{Broadband backbone network}

The provision of broadband services through local access networks to final customer premises requires backbone networks able to connect customers to the Internet. As of now, the fiber optic technology is the most cost effective backbone technology to reach densely populated areas, which are the most profitable markets for broadband services. In Peru, most of these areas are situated along the coastal region. The lack of fiber optic backbone in most of the Andes and Amazon regions (due to the harsh geography and consequently high deployment cost) has been a critical limiting factor for the provision of broadband services in these 
regions. Figure 3 shows the distribution of backbone technologies in the different states: fiber optic in the coast, microwaves in the Andes and satellite in the Amazon. Based on bandwidth capacity, microwave and satellite technologies are able to provide only a small fraction of the bandwidth provided by fiber optic technology. More backbone bandwidth allows the delivery of more broadband services to customers, and as a result, broadband deployments are limited or minimal in the Andes and Amazon regions. Figure 3 shows mobile and fixed broadband coverage in the different states in these regions. We can observe that most mobile and fixed broadband coverage occurs in the coastal states where the fiber optic backbone exists.

As part of the Peruvian National Broadband plan, the Ministry of Transportation and Communications (MTC) and the Investment Fund in Telecommunications (FITEL) have designed and planned the deployment of the nation-wide fiber optic backbone which will reach 180 provinces in 22 states in the coastal, Andes and the Amazon regions, and will be completed by the middle of 2016 [17]. In Peru, states are divided into provinces, provinces into districts, and districts into towns. The network will reach the capitals of provinces. Additional transport and access networks will be deployed to reach final customers in districts and towns. Figure 4 shows the national fiber optic backbone in 2011 and the expected backbone by June 2016. In 2014, the telecommunications company TV Azteca, won the Peruvian universal service bid (USD 323 Million) to deploy the nationwide backbone network. 


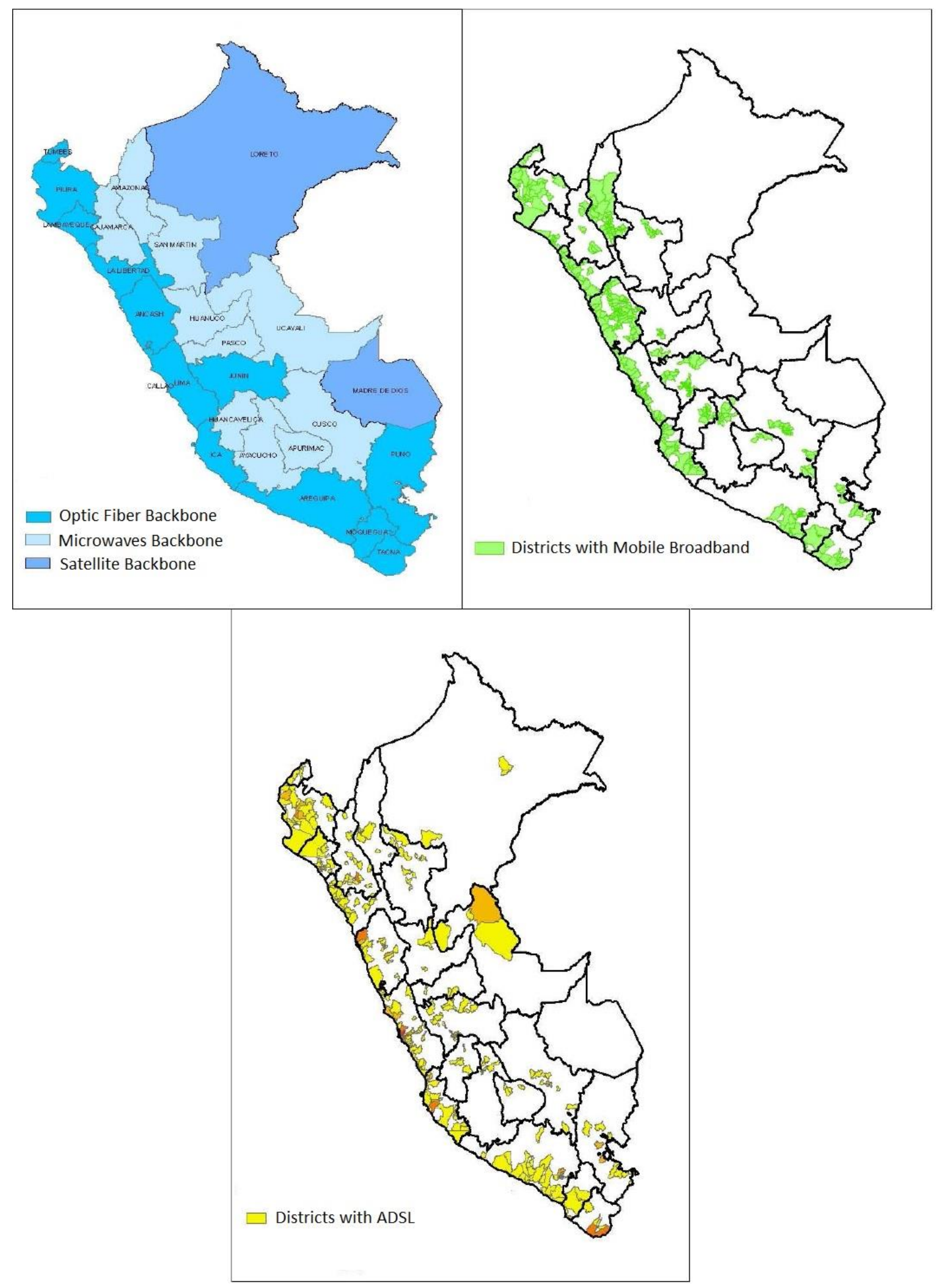

Figure 3. (Left) Distribution of backbone technologies by states. (Right) Mobile broadband coverage. (Bottom) Fixed broadband coverage [14]. 


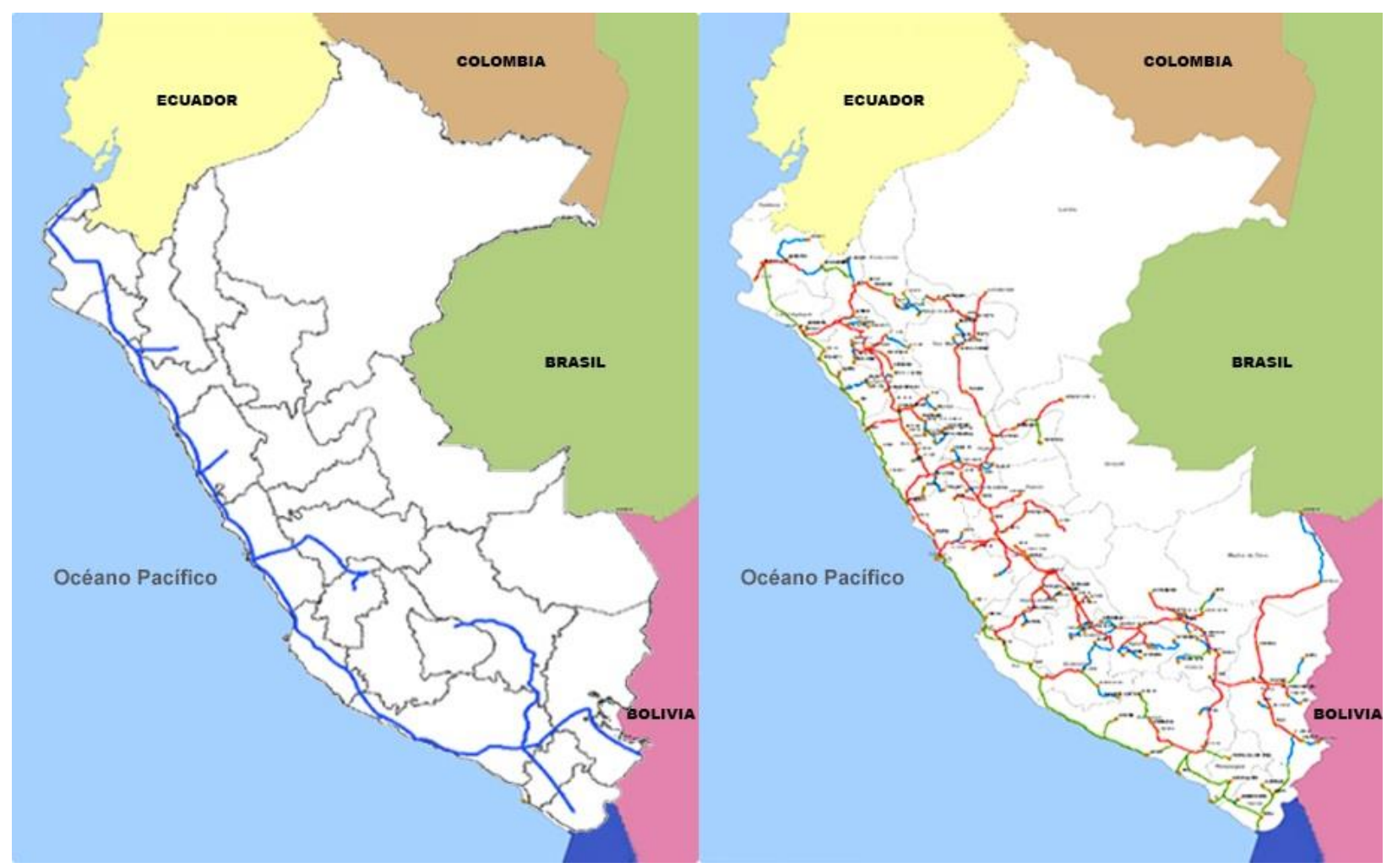

Figure 4. Fiber optic backbone in 2011 (left) and backbone in 2016 (right) [4].

Once the fiber optic backbone is deployed, the next big challenge that the government and broadband service providers will face under the national broadband plan is to reach final customers, especially in areas where broadband legacy networks are minimal or non-existent today. The deployment of the fiber optic backbone reaching capital of provinces in the Coastal, Andes and Amazon regions will reduce the operators' costs for providing broadband services. The next step to provide broadband services to final customers will be carried out through transport networks (using fiber optic links from the capitals of provinces to the capitals of districts) and local access networks (using wireless links from the capital of districts to towns). Beginning in 2014 and during 2015, the Peruvian government set public bids for the deployment of broadband networks (fiber optic 
transport network and wireless local access network) in eight states (each project or bid corresponds to one state), where broadband service providers will compete for a government subsidy to provide fixed broadband services to government institutions (mandatory or primary customers), and business and residential customers (secondary customers) [18] [19]. Appendix 1: FITEL Projects and Bids for Broadband Deployments presents more detailed information on the granted, in progress and future broadband deployment bids for the 22 states. Details on the regulatory environment set for the transport and access networks are provided in Section 2.4 Regulatory and Policy Environment for

\section{Providing Broadband Services in Peru.}

This study focusses on the deployment and operation of local access networks in unserved and underserved areas in rural settings in the Andes and Amazon regions in Peru. We analyzed current and new wireless technologies and platforms for the local access networks. The next section presents a description of such technologies and platforms.

\subsection{Wireless Broadband Technology Options for Rural Areas}

The access networks to be deployed in the context of the Peruvian national broadband plan will extend from the capitals of districts to towns providing fixed broadband service to final customers. The targeted final customers include government institutions (i.e., schools, health posts, police stations.) as anchor customers, and business and residential customers. The use of wireless 
technologies for access networks, especially in rural areas, is supported by several studies which conclude that wireless technologies are the first viable infrastructure for new deployment to provide broadband services to underserved and unserved rural areas [20] [21] [22]. Wireless technologies can be deployed much faster with less initial investment, offer more flexibility in terms of adapting to changing bandwidth requirements, and are more efficient in terms of network management and operation. Wireless technology options which can be deployed in access networks to provide fixed broadband services include the following: Wi-Fi, LTE, WiMAX, TV white spaces, milimeter-wave and satellite. These technologies, except for the satellite technology, are commonly deployed in terrestrial local access networks. This study also includes analyzing a subset of these technologies known as stratospheric access networks deployed using new and innovative platforms such as unmanned aerial systems (i.e. super-pressure balloons and drones). The following subsections describe the wireless technologies and broadband deployments using these technologies in rural areas in countries around the world.

\subsubsection{Wi-Fi}

The IEEE 802.11 standard, or Wi-Fi certified by the Wi-Fi alliance, operates in the $2.4 \mathrm{GHz}$ and $5.8 \mathrm{GHz}$ ISM unlicensed bands and can achieve data rates up to 11Mbps (802.11b), 54Mbps (802.11a/g) and 600Mbps (802.11n). For the highest data rate profiles, Wi-Fi uses orthogonal-frequency division multiplexing (OFDM) carriers in $20 \mathrm{MHz}$ and $40 \mathrm{MHz}$ channels, and multiple-input and multiple-output (MIMO) antennas, among other features. More technical details of the Wi-Fi 
standard can be found in [23]. Wi-Fi was designed for wireless local area networks (WLANs) to enable communication among devices and stations in a range from tens to a few hundred meters. Using Wi-Fi equipment in a default configuration for longer distances can lead to unpredictable link stability and a drop in data rate performance. To extend the coverage and achieve high performance over longer radio links, research groups and equipment manufactures developed low cost, long range solutions [24] [25] [26] [27]. Solutions for the physical layer include simply incorporating high gain antennas in the access point (AP) or customer premise equipment (CPE). Another option is to modify (in most cases using open-source software) acknowledgement and timeout frames. This expansion of the original coverage of Wi-Fi equipment and using unlicensed spectrum has led to a greater adoption of this technology, especially in rural areas of developing countries. Wi-Fi deployment often is a cheaper solution than other wired and wireless technologies to provide broadband and Internet service in these areas.

Wi-Fi technology has been used in both developed and developing countries for providing broadband and Internet services to customers in rural areas. For example, Airjaldi is a class A Internet service provider (ISP) which provides broadband connectivity in rural India [28]. Airjaldi has deployed ten Wi-Fi networks in six states in India and serves large and small corporate, civil society and private customers. One of Airjaldi's networks is located in Dharamsala and provides Internet service to a $70 \mathrm{~km}$ radius area. Another Wi-Fi deployment in a rural area is the Nepal Wireless Networking Project (NWNP) which provides 
broadband Internet service to 175 villages in 15 districts in a mountainous region in Nepal [29]. In the Airjaldi and NWNP cases, Wi-Fi nodes were mounted on low masts placed on rooftops or high places to reduce deployment costs (cheaper than building tall towers). The Wi-Fi radio solutions also use low-cost and reliable commercial routers or low-cost customized hardware solutions which operate in the $2.4 \mathrm{GHz}$ and $5.8 \mathrm{GHz}$ bands. The network equipment is mostly solar-powered due to the lack of reliable power supply in these rural mountainous areas. Other reported commercial Wi-Fi networks in mountainous rural areas providing broadband Internet services are the Digital Gangetic Plains (DGP), Ashwini, and Aravind networks in India [24] [25]. Other Wi-Fi networks have also been deployed in rainforest (dense vegetation) settings in the Peruvian Amazon region along the Napo River [30] and in the Balsapuerto District [31]. Although these two networks mostly provide broadband Internet services to health posts and hospitals for telemedicine purposes, government network managers recognize that under the proper business model and regulatory framework, these networks could also provide broadband Internet services to other customers. In these rural deployments (both in mountainous and rainforest areas), the Wi-Fi solutions are characterized for being robust (i.e., against extreme weather conditions and power fluctuations), technical viable and affordable.

\subsubsection{WiMAX}

The IEEE 802.16 standard, or Worldwide Interoperability for Microwave Access (WiMAX), emerged as a high capacity and long range technology (up to 48 
$\mathrm{km}$ ) for the provision of fixed and mobile broadband services for wireless metropolitan area networks (WMAN) achieving data rates up to 350Mbps. Fixed WiMAX, or the 802.16-2004 and 802.16d standards, is based on OFDM for the air interface and time-division multiple access (TDMA) for the multiple access among client stations. Mobile WiMAX, or 802.16e-2005, is based on orthogonal frequency division multiple access (OFDMA) for the air interface. This standard was one of the technologies chosen by the ITU for providing IMT-Advanced services or $4 \mathrm{G}$ mobile services. WiMAX operates in the licensed bands $2.3 \mathrm{GHz}, 2.5 \mathrm{GHz}$ and 3.5 $\mathrm{GHz}$ and the unlicensed band $5.8 \mathrm{GHz}$. It uses different bandwidths profiles $(1.75 \mathrm{MHz}, 3.5 \mathrm{MHz}, 5 \mathrm{MHz}, 7 \mathrm{MHz}, 10 \mathrm{MHz}$ and $20 \mathrm{MHz}$ channels), time-division duplexing (TDD) and frequency-division duplexing (FDD) schemes, among other features. More technical details of WiMAX can be found in [23].

WiMAX can operate in backhaul or local access network radio links and was proposed by the WiMAX Forum as an alternative to provide broadband services in suburban and rural areas. For example, in suburban and rural areas in Spain, Iberbanda offers broadband services (Internet access, VoIP service, data transmission, etc.) to residential, small and medium businesses, and local government offices using a nationwide WiMAX license in the $3.5 \mathrm{GHz}$ band [32] [33]. Using a licensed band allows Iberbanda to compete in terms of quality of service against landline broadband providers. This company has one of the largest operating WiMAX networks in Europe using high capacity Ethernet microwave point-to-point links for the backhaul while using WiMAX base stations for access 
nodes. Another commercial WiMAX network was deployed by the broadband provider Packet One (P1) in Malaysia to serve underserved and rural areas in Peninsular Malaysia [34]. In 2010, P1 completed a WiMAX network using the 2.3GHz band to provide service to 3,500 residential customers [35]. WiMAX networks have also been deployed in developing countries as joint projects among multiple organizations (local, international, public and private), such as the case of a network operating in the remote mountainous province of Lao Cai in Vietnam. This network provides broadband Internet connectivity and VoIP telephone service to 11 locations such as a post offices, healthcare clinics, schools and local guest houses [34] [36]. In South America, WiMAX networks have been deployed in the Brazilian rainforest in the Ceara and Para States using a public-privatepartnership framework. WiMAX base stations provide last mile broadband services to customer in rural towns [37] [38]. The WiMAX local access network interconnects to aerial fiber optic backhaul deployed along electric power grid lines, which reach major urban areas and some rural areas. In the Ceara State's WiMAX network, the $3.5 \mathrm{GHz}$ band could not be used due to a pending legal dispute among operators and the national regulatory agency. Thus, this WiMAX access network uses the unlicensed $5.4 \mathrm{GHz}$ and $5.8 \mathrm{GHz}$ bands, in addition to the $4.9 \mathrm{GHz}$ band which was allotted for public safety use in Brazil (used by government agencies for communications). In the WiMAX network in the Para State, WiMAX nodes operate in the $5.8 \mathrm{GHz}$ unlicensed band. These two networks serve schools, state and municipal agencies, hospitals, health centers, police stations, among other 
government institutions. Each WiMAX node serves customers in a radius from $3.2 \mathrm{~km}$ to $16 \mathrm{~km}$ using high-gain directive antennas in the CPE.

\subsubsection{LTE}

Long Term Evolution (LTE) was designed by the 3rd Generation Partnership Project (3GPP) group for mobile communications in densely populated areas and can achieve data rates up to hundreds of Mbps. The 3GPP group has released several versions of the LTE standard, from the first release (Rel-8 in 2008) to LTE-Advanced (Rel-11). LTE was one of the technologies chosen by the ITU for providing IMT-Advanced services or $4 \mathrm{G}$ mobile services, and operates in the same bands as its predecessor GSM, in addition to other bands. Most common LTE bands range from $700 \mathrm{MHz}$ to $3.8 \mathrm{GHz}$. A complete list of the 44 bands can be found in [39]. LTE is based on OFDMA for the air interface, and uses different bandwidth profiles $(1.4 \mathrm{MHz}, 3 \mathrm{MHz}, 5 \mathrm{MHz}, 10 \mathrm{MHz}$ and $20 \mathrm{MHz}$ channels), MIMO, convolutional and turbo codes, and adaptive modulation, among other features. More technical details of LTE can be found in [23].

LTE has increasing penetration in both developed and developing countries. In developing countries, the $4 \mathrm{G}$ coverage by 2014 reached around $10 \%$ of the population. LTE has also been used to provide fixed broadband services in rural areas. In the United States, the assignment of the $700 \mathrm{MHz}$ band (digital dividend) allowed operators to expand LTE services across the country including rural areas (98\% of the population coverage by the end of 2014). For example, Verizon's LTE in Rural America Program and Sprint's NetAmerica Alliance covered 2.2 million and 
34 million people, respectively, by the end of 2014 , which is around $11 \%$ of the United States population. In these programs, rural and regional carriers lease $700 \mathrm{MHz}$ and AWS (1.7/2.1GHz) spectrum from Verizon and Sprint (nationwide carriers) to deploy their own $4 \mathrm{G}$ networks [40]. Other LTE deployments in rural areas in the United States were carried out by the satellite-based services operator Dish Networks which partnered with nTelos and Sprint to provide 4G LTE (TDD) fixed wireless broadband services to Virginia and Corpus Christi in Texas. In both areas, the broadband service operates in the $2.5 \mathrm{GHz}$ band and uses a ruggedized outdoor router with built-in high-gain antennas to provide speeds of up to $10 \mathrm{Mbps}$ per customer [41] [42]. In Australia, the government established the National Broadband Network (NBN) Co and appointed Ericsson to build and operate a 2.3GHz fixed-wireless broadband network based on LTE to serve rural and regional households in Australia offering 12Mbps download data rate and 1Mbps upload data rate per customer [43]. This deployment must be completed by the end of 2015. In the United Kingdom, the largest mobile operator Everything Everywhere (EE) is carrying out rural wireless LTE $4 \mathrm{G}$ rollouts in the $1,800 \mathrm{MHz}$ band to provide voice and data services to 1,500 communities across the UK, including hard to reach areas and underserved communities [44]. In South America, the Colombian operator Avantel is currently deploying Nokia LTE networks in major cities and rural areas to VoLTE using small cell solutions, becoming the first commercial LTE small cell network in Colombia [45]. 


\subsubsection{TVWS}

White Spaces can be defined as part of the spectrum, available for a radio communication application at a given time in a given geographical area on a noninterfering/non-protected basis with regard to primary and other services. The Television White Spaces (TVWS) technology, IEEE 802.11af or IEEE 802.22 standards, operates in white spaces (i.e., unused frequencies) of the broadcast television (from 54MHz to $862 \mathrm{MHz}$ ) spectrum. The 802.11 af standard, also known as White-Fi or Super Wi-Fi, was designed for wireless local area networks (WLANs) with a range up to a few kilometers and has bandwidth profiles of $5 \mathrm{MHz}$, $10 \mathrm{MHz}, 20 \mathrm{MHz}$ and $40 \mathrm{MHz}$ [46]. The 802.22 standard was designed for wireless regional area networks (WRANs) with a range up to several tens of kilometers and has bandwidth profiles of $6 \mathrm{MHz}, 7 \mathrm{MHz}$ and $8 \mathrm{MHz}$ [46]. Both standards use OFDM for the air interface to achieve data rates of a few tens of Mbps, and utilize cognitive radio features and geolocation databases for dynamic spectrum access. Currently used geolocation databases are managed and maintained by the White Space Database Administration Group (formed by companies such as Microsoft, Google, Spectrum Bridge and Telecordia) and provide information of location, used spectrum and other technical specifications of RF equipment [47]. The TVWS technology has the advantage of enhanced propagation features due to the use of frequencies bellow $1 \mathrm{GHz}$ allowing for the penetration of walls and other obstacles.

In developing countries, measurement campaigns have shown that TV broadcasting spectrum is mostly unused in sparsely populated areas and there is 
not enough return on investment for broadcasters to offer simultaneous TV channels [48]. Then, the TVWS technologies can offer an alternative to providing broadband service to these areas. The Dynamic Spectrum Alliance (DSA), which is a global organization advocating for laws and regulations that will lead to more efficient and effective spectrum utilization, lists in its website several completed and ongoing pilot projects related to TV White Spaces deployments around the world [49]. For example, early deployments have been reported in rural areas in Bhutan, Philippines, Taiwan, Botswana, Namibia, Ghana, South Africa, Malawi, Kenya, Scotland, United Kingdom, United States, and Uruguay. Often these pilots are mainly conducted as joint efforts among government organizations (in education, healthcare, agriculture, commerce, etc.), regulatory agencies, and technical partners. Some technical partners are telecom operators, TVWS equipment vendors, universities, research institutes and research initiatives from companies such as Microsoft and Google. The first TVWS pilot in Latin America is conducted in Uruguay. This project provides broadband access to ten rural schools.

\subsubsection{Gigabit Satellite}

This type of broadband technology is currently offered by the company O3b which stands for "Other 3 Billion". This company provides satellite communication backhaul service offering low latency (less than $150 \mathrm{~ms}$ ) and data rate comparable to fiber optic technology [50]. O3B has a constellation of 12 satellites with a ground period of 360 minutes ( 4 contacts per day) using the Ka-band providing a coverage of 45 degrees north/south latitudes. The satellites provide 10 beams per region ( 7 
regions) totaling 70 remote beams for the entire constellation. The latency is less than 150 milliseconds due to the use of Medium Earth Orbit (MEO) satellites (8,062km above Earth). The maximum available throughput per beam (over a single transponder) is $1.6 \mathrm{Gbps}$ (800Mbps x 2) and each beam provides coverage of $700 \mathrm{~km}$.

O3B currently offers the following broadband services: 1) IP trunking, 2) mobile backhaul, 3) energy stations and platforms, 4) maritime communications, and 5) government and other agency communications. These services are available in Latin America, Africa, the Middle East, Asia and the Pacific, and have customers in Solomon Islands, Federal States of Micronesia, Palau, East Timor, Caribbean, Democratic Republic of the Congo, Papua New Guinea, South Sudan, Norfolk Island and Madagascar.

\subsubsection{Millimeter-wave}

Technology operating in millimeter waves is currently used for high speed backhauling (point to point) to connect cell sites or access points to provide high speed Internet. There is assigned unlicensed and licensed spectrum for this technology. The unlicensed millimiter-wave spectrum operates in the $60 \mathrm{GHz}$ band (57-66GHz), offering $7 \mathrm{GHz}$ of bandwidth, and the licensed millimiter-wave operates in the $71-76 \mathrm{GHz}$ and $81-86 \mathrm{GHz}$, offering bandwidth from 10 to $80 \mathrm{MHz}$, although for shorter distances. Equipment working on these bands benefit from heavy atmospheric absorption, which limits range of operations but also unwanted 
interference. Millimeter-wave can achieve up to $2.5 \mathrm{Gbps}$ for a $1 \mathrm{Km}$ point to point radio link [23] [51].

\subsection{New unmanned aerial systems for stratospheric wireless access networks}

\subsubsection{Unmanned super-pressure balloons: Project Loon}

Delivering broadband services using super-pressure balloons travelling in the stratosphere is a Google $\mathrm{X}$ initiative known as the Project Loon [52]. This project will allow customers in rural and remote areas to connect to the balloons network (in the stratosphere $20 \mathrm{~km}$ above the ground) and then to the Internet. In the stratosphere, twice as high as airplanes and the weather, there are many layers of steady winds which vary in direction and speed. The balloons will travel to the needed location or route by rising or descending into a layer of wind blowing in the desired direction. Project Loon chose super-pressure balloons to keep the volume and altitude relatively stable in order to avoid the problem of descending at night when the gas inside the envelope cools down. To provide the broadband service, the signal from customer's devices connect to a balloon network in the stratosphere, and then down to the global Internet on Earth, just as satellite service works at much higher latitudes. The active payload that the balloons carry consists of subsystems including computational, solar energy, navigation, sensing, altitude control and telecommunications [53]. The telecommunications subsystem includes free space optical (FSO) and radio frequency (RF) equipment for the 
backhaul and access radio links. For the access radio links, the Loon project has conducted tests in rural areas in New Zealand, California and Brazil using both the ISM $(2.4 \mathrm{GHz}$ and $5.8 \mathrm{GHz})$ and LTE bands [52] [54] [55]. In 2014, the Loon project expressed their interest on using LTE bands and working in partnership with operators and telcos in different countries due to the potential to achieve universal access targets mandated by countries in a more cost-effective manner than deploying terrestrial wireless networks, cell towers and fiber optic to remote rural regions. Some advantages of using this approach over terrestrial or satellite networks are the following: 1) it is a more dynamic platform than satellite, 2) its radio coverage of $20-50 \mathrm{Km}$ fits between satellite spot-beam and macro cell mobile platforms, 3) it targets rural, peri-urban settings using a direct-to-consumer model; and 4) its regulatory framework is less constraining than that of satellites [7]. In tests, balloons using LTE provided 2MBs to a ground antenna and 5Mbps to a handset [55]. More details on the description of the Project Loon and previous related initiatives (airships), and our analysis on the operation and coverage of the balloons network and the estimated costs can be found in Appendix 2: High

\section{Altitude Platforms (HAPs) for Providing Broadband Services.}

In 2013, the Project Loon started carrying out flying and coverage tests in several countries. In one of the tests, a balloon was able to fly from New Zealand to Latin America and back delivering Internet connection along the route. For these tests, the Loon project has worked with telcos such as Vodafone in New Zealand, Telstra in Australia, and Telefonica, Vivo and Telebras in Latin America [52] [54] 
[55], and the project announced plans to work with more countries such as India, Indonesia and Sri Lanka [56] [57] [58] [59]. A potential business model is to partner with governments and operators which already have LTE spectrum and an operator business (infrastructure, sales and billing departments, etc.), so Google can obtain permission to use the spectrum and provide the balloons as cellular towers in the sky. In this way, all the partners can share the revenue for each new customer reached in rural areas [54]. In February 2016, balloons entered Sri Lanka's air space for service coverage testing. It has been announced that the government will take 25 percent stake in the balloon-powered Internet program by providing the required spectrum, and that local operators partnerships will take a 10 percent stake [56] [57].

\subsubsection{Unmanned aircrafts: Google and Facebook solar-powered drones}

The initiative of using unmanned solar-powered aircrafts flying at $20 \mathrm{~km}$ above the ground to provide Internet service in unserved areas is currently led by Google and Facebook. In April 2014, Google acquired Titan Aerospace [60] and started the Project SkyBender, which is currently testing in New Mexico Titan's high-altitude-solar-powered drones using millimeter wave technology to transmit at data rates higher than 4G LTE [61] [62]. In March 2014, Facebook acquired Ascenta [63], UK-based drone manufacturer, and is also currently testing solarpowered drones using free space optics (high-powered laser beams) to achieve high data rate transmissions [64] [65]. Both Google and Facebook approaches to achieve high data rates using narrow beam and high frequency technologies (millimeter- 
wave and lasers) indicates that the drones will work as a backhaul network. The idea of using unmanned aerial vehicles (UAVs) or drones to deploy a backhaul network is innovative and promising, but it is still in a design and testing stage with no current commercial deployments. More technical details on the UAVs currently under testing by Google and Facebook can be found in Appendix 2: High Altitude Platforms (HAPs) for Providing Broadband Services.

\subsection{Regulatory and Policy Environment for Broadband Services in Peru}

This subsection describes the relevant regulatory and policy context for the Peruvian national broadband plan and for our study on the deployment and operation of local access networks to introduce broadband services in rural areas in the Andes and Amazon regions of Peru. First, we introduce the government agencies in Peru in charge of setting the telecommunication regulatory and policy framework:

- Executive and policy agency: Ministry of Transportation and Communications (MTC)

- Telecom regulator: Private Investment in Telecommunications Supervision Agency (OSIPTEL)

- Universal service agency: Investment Fund in Telecommunications (FITEL)

- National investment and funding agency: Private Investment Development Agency (PROINVERSION) 
- Other agencies: National Office for Electronic Government and Information (ONGEI)

In 2010, the MTC designed and released the "National Plan for Broadband Development in Peru 2010-2016" to expand broadband services and increase penetration on a nationwide basis with emphasis in rural areas. The funding model chosen for this national plan was developing public-private-partnerships (PPP). The main objectives of this first national broadband plan include the following [14]:

- $100 \%$ Internet penetration at a minimum speed $2 \mathrm{Mbps}$ in all government institutions in urban areas. (i.e., schools, healthcare facilities, police stations, among other government offices.)

- $100 \%$ Internet penetration nationwide in municipalities, schools and healthcare facilities in all districts.

- 4 million broadband connections nationwide.

- 0.5 million broadband connections at data rates higher than 4 Mbps.

- Nationwide fiber optic backbone deployment. Auction winner (2013): TV Azteca (Salinas Group). USD 323 Million.

In July 2012, the Peruvian Congress, following recommendations of the national broadband plan, passed the N 29904 Bill for the Development of Broadband and Deployment of the National Fiber optic Backbone Network. The bill included the following titles:

- Title I : General regulations

- Title II : Essential infrastructure for the development of broadband 
- Title III : Content generation, applications and capability training

- Title IV : Competent organizations for the development of broadband

- Title $\mathrm{V}$ : Infringement and sanction regime

A year later in November 2013, the President approved the N 014-2013MTC

Supreme Decree for Regulation of the Bill for the Development of Broadband and Deployment of the National Fiber Optic Backbone Network. This decree provided specific regulations and policies for execution of the initial bill. The following subsection presents relevant aspects of the bill and supreme decree for the deployment of the national fiber optic backbone, and the transport and access networks.

\subsubsection{National fiber optic backbone network and transport and access networks}

The N 29904 Bill and the N 014-2013MTC Supreme Decree states the framework and regulation for the development of broadband and the deployment of the national fiber optic backbone network, which is the first and critical piece to launch broadband services on a nationwide basis. Regarding the deployment of this backbone network, the bill and the supreme decree define the main following points:

- The MTC is in charge of the network on behalf of the Peruvian government and is authorized to grant the network in concession to private operators for construction, operation, maintenance and use of the network. However, the Peruvian government keeps ownership of the network in all cases. 
ProInversion is the agency in charge of setting an open public bid for granting concession of the network.

- The private operator awarded with the concession of the network to provide public telecom services will be subject to rate regulation, activities monitoring, and other duties that OSIPTEL will define to prevent anticompetitive behavior as a result of the integrated vertical structure of private operators in the telecom industry.

- The operation of the network will be in charge of one or more operators. The operator must request to the MTC an exclusive license to provide public telecom services and register only the carrier service. The network operator must provide service only to other telecom operators and not to final customers. Additionally, to prevent any anticompetitive conduct and vertical foreclosure agreements, OSIPTEL will oversee the fulfilling of telecom carrier duties.

- The operator of the network will be allowed to use current and new energy, hydrocarbon, railroad and road infrastructure and facilities, as well as infrastructure of government-owned companies. Future infrastructure deployments in the case of the mentioned industries should also include pipes and chambers for fiber optic roll-outs. The government will cover the marginal cost for this additional fiber optic infrastructure and will hold the ownership. 
- The network will interconnect to international points of presence in one end (international carriers), and will reach capitals of districts in the other end through deployment of regional networks.

- FITEL will be authorized to design and fund broadband network deployments in the district level. Regional (State) governments are allowed to participate in funding these projects in their own jurisdictions.

- FITEL is authorized to fund solely telecommunications services and the required infrastructure to provide these services in rural or high priority social interest areas.

In 2013, the company TV Azteca won the MTC and ProInversion's government bid (USD 323 Million) to deploy (with completion scheduled by the end of 2016), operate and use the national fiber optic backbone network for a 20 year period. The provision of broadband services to reach final users will be carried out through transport and access networks connected to this fiber backbone, which will reach only capitals of provinces.

In 2014 and 2015, FITEL and ProInversion set open bids for the deployment of broadband networks (fiber optic transport network and wireless access network) in eight states (each project or bid correspond to one state), where broadband service providers competed for a universal service subsidy to provide fixed broadband services to government institutions (mandatory or primary customers), and business and residential customers (secondary customers) [18] [19]. Three major broadband service providers were awarded the concession of the broadband 
networks in these eight states: Gilat (four states), Telefonica (1 state) and Redes Andinas (2 states).

This study focusses on the access network segment and comparing the costs of different wireless technology options to provide broadband services to final customers in rural areas in the Andes and Amazon regions. In Chapter 3:

Research Methodology, the technical and engineering cost analysis is described assuming the current regulation and policy set by the national broadband plan for the deployment and operation of access networks, and current spectrum regulation set for bands used by the analyzed technologies. Another important factor we also include in the analysis is access network requirements set by FITEL in the government bids regarding to technical specifications (for telecom and energy systems), and infrastructure requirements, among others.

\subsubsection{Spectrum regulation}

In 2005, the MTC approved and updated a new the National Spectrum Allocation Plan [66]. In the last 10 years, modifications and amendments were added to the plan, recommended to the MTC by multi-sectorial commissions and committees, to refarm and make more efficient and productive use of the spectrum in order to promote expansion of new networks and services across the country. This subsection describes the current allocation and assignment of spectrum for providing public telecommunication services to final customers using the following wireless technologies: Wi-Fi, TV White Spaces, WiMAX and LTE. 


\section{Wi-Fi bands}

In 2008, the MTC allocated the following bands to providing public telecommunication services in rural or underserved areas without requiring any license or authorization: i) $902-928 \mathrm{MHz}, 2,400-2,483.5 \mathrm{MHz}$, and $5,725-5,850 \mathrm{MHz}$ (maximum transmission power of $30 \mathrm{dBm}$ ), and ii) $5,250-5,350 \mathrm{MHz}$ and $5,470-$ $5,725 \mathrm{MHz}$ (maximum transmission power of $24 \mathrm{dBm}$ ) [67]. There were no restrictions for antenna gains. In 2011, the MTC modified this allocation and reserved the $902-915 \mathrm{MHz}$ band for licensed public telecommunication services [68]. In 2013, the MTC updated the frequency ranges and maximum power limits for equipment in the unlicensed bands to provide public communication services in rural areas as follows: i) $915-928 \mathrm{MHz}$ (maximum EIRP of $30 \mathrm{dBm}$ ), ii) $916-928 \mathrm{MHz}$, $2,400-2,483.5 \mathrm{MHz}$, and $5,725-5,850 \mathrm{MHz}$ (maximum transmission power of $30 \mathrm{dBm}$ ), and iii) $5,250-5,350 \mathrm{MHz}$, and $5,470-5,725 \mathrm{MHz}$ (maximum transmission power of $24 \mathrm{dBm}$ ) [69]. There were no limits for antenna gains. The use of these unlicensed bands allows operators to provide public telecommunication services in rural areas without incurring any spectrum license fees or charges.

\section{Television bands}

The TVWS technology will use unused channels (white spaces) in the broadcast television spectrum. In 2006 , the MTC allocated the $470-584 \mathrm{MHz}$ band for digital television broadcast which uses $6 \mathrm{MHz}$ channels, including two channels

for trials and testing [70]. One year later, two more channels were added for trials and testing [71]. In 2009, the MTC expanded the spectrum range for digital 
television and allocated the $470-608 \mathrm{MHz}$ and $614-746 \mathrm{MHz}$ bands, and ended assignment of analog broadcast television channels in these bands [72]. In 2010, the MTC updated broadcast television (allowing both digital and analog channels) bands allocation to the following frequency ranges: $470-608 \mathrm{MHz}$ and $614-698 \mathrm{MHz}$ [73]. This modification reserved the $698-806 \mathrm{MHz}$ band for fixed and mobile services, which was auctioned in 2015 .

\section{WiMAX bands}

In 2005, the MTC allocated the $3,400-3,500 \mathrm{MHz}$ and $3,500-3,700 \mathrm{MHz}$ bands (also collectively called the $3,500 \mathrm{MHz}$ band), as primary use, to fixed access public telecommunication services [66]. The MTC also set a spectrum cap regulation which stated that each operator (including affiliated and partner companies) can acquire spectrum licenses in these bands for a maximum of $50 \mathrm{MHz}$ in any geographic area [74]. In 2006, the MTC auctioned, through the government agency ProInversion, blocks in these bands for Lima, and granted charge-free licenses for other geographic areas than Lima in a first come, first served basis [75]. The operators Telefonica Moviles, America Movil, Entel (former Nextel) and Americatel currently hold spectrum licenses in the $3,500 \mathrm{MHz}$ band [76] [77].

In 2007, the MTC began the refarming of the $2,500-2,692 \mathrm{MHz}$ band, which was allocated, as primary use, to public telecommunication services [78]. The MTC's advisory board recognized the potential of this band to provide fixed and mobile services as this band has been used in other countries to provide WiMAX and IMT-Advanced services. The refarming required previous licensees of this 
band to give back certain amount of unused spectrum, and to free the 2,668$2,692 \mathrm{MHz}$ band (by migrating to the $2,500-2,668 \mathrm{MHz}$ band). The refarming aimed to promote expanding networks and services to rural and underserved areas using new technologies [78]. In 2010, the MTC auctioned the $2,668-2,692 \mathrm{MHz}$ band to Yota del Peru for USD 3.9 Million [79].

\section{LTE bands}

In Peru, the MTC has applied since 2005 a spectrum cap regulation in order to develop a competitive public mobile telecommunication services market (i.e. mobile telephone and personal communication services) which would benefit consumers. The spectrum cap regulation states that each operator (including affiliated and partner companies) can acquire spectrum licenses for a maximum of $60 \mathrm{MHz}$ [80] to provide public mobile telecommunication services. There are additional regulations to limit the number of licenses per operator in specific bands. For example, operators are not allowed to have two licenses (each license is a block) in the $800 \mathrm{MHz}$ band. This spectrum cap applies to the following bands: $700 \mathrm{MHz}, 800 \mathrm{MHz}, 900 \mathrm{MHz}$, and 1,900MHz [76].

The spectrum cap for public mobile telecom services has been identified in the National Broadband Plan, in 2010, as a barrier for the deployment and expansion of broadband services nationwide in both urban and rural areas [81]. As a result, the plan recommended developing new policies and regulations based on technology evolution, global and local market trends, and efficient use of the spectrum based on national interests. In recent years, the government through the 
MTC started making more spectrum available to provide broadband services, and developing regulation to increase broadband and Internet penetration.

By early 2011, in Peru, operators provided public mobile services in the $800 \mathrm{MHz}$ and $1900 \mathrm{MHz}$ bands. Later in 2011, the MTC allocated additional bands to public mobile communication services to promote deployment and expansion of mobile broadband services. The allocated bands were the following: $894-902 \mathrm{MHz}$ and $939-947 \mathrm{MHz}, 902-915 \mathrm{MHz}$ and $947-960 \mathrm{MHz}$ [68], and $1710-1770 \mathrm{MHz}$ and 2110-2170MHz [82]. In August 2012, the MTC auctioned the 902-915MHz and $947-$ 960MHz bands to Viettel for USD 48.4 Million [83]. In July 2013, the MTC auctioned two blocks of the $1.7 / 2.1 \mathrm{GHz}$ band for $4 \mathrm{G}$-LTE services to Telefonica del Peru (Movistar) and Americatel (Entel), for USD 152.23 Million and USD 105.51 Million respectively [proinversion-2100]. Before this latter auction, the MTC modified the $60 \mathrm{MHz}$ spectrum cap regulation since most operators were at or close to reaching the cap. The new spectrum cap regulation states that the $60 \mathrm{MHz}$ spectrum cap per operator applies only for the $800 \mathrm{MHz}, 900 \mathrm{MHz}$ and $1,900 \mathrm{MHz}$ bands, and a different $40 \mathrm{MHz}+40 \mathrm{MHz}$ spectrum cap applies for the $1.7 / 2.1 \mathrm{GHz}$ band [80]. For the auction of the 1.7/2.1GHz 4G-LTE band, the MTC applied this new spectrum cap regulation. For 2015, the MTC, through ProInversion, will auction 3 blocks (each block $15 \mathrm{MHz}+15 \mathrm{MHz}$ ) of the $698-806 \mathrm{MHz}$ (called $700 \mathrm{MHz}$ ) band. 


\section{CHAPTER III}

\section{RESEARCH METHODOLOGY}

This chapter presents the research methodology used to address the research question, subproblems and hypotheses introduced in Chapter 1 as follows:

Research Question: What is the roadmap for introducing broadband services to unserved and underserved areas in the Andes and Amazon Regions of Peru?

\section{Subproblems:}

1 What are the current leading wireless technology options to consider for providing broadband services in underserved and unserved areas of developing countries, and how can they be designed for deployment in this setting?

2 How do the deployment and operating cost for this set of wireless technology options compare with each other, and what is the optimal roadmap for deployment of these technologies over the next 10 years?

3 What are the policy implications as a result of this detailed engineering economic analysis of wireless broadband technology options? 


\section{Hypotheses:}

1 Deploying wireless access networks in the Andes and Amazon regions of Peru using the Wi-Fi technology and unlicensed spectrum has the lowest deployment and operating cost in a 10-year period.

2 Wireless access networks using stratospheric platforms (i.e. stratospheric balloons and unmanned aerial vehicles) in the Andes and Amazon regions of Peru has the lowest deployment and operating cost for the initial years but are not able to scale for a 10 -year period to serve forecasted customer and speed growth.

3 Spectrum management and regulation have a quantifiable technology and economic impact in the deployment and operation of wireless access networks in the Andes and Amazon regions of Peru and are important to define the best technology fit to introduce broadband services in these regions.

This study conducts technical and engineering cost analyses to evaluate current and new disruptive wireless technologies (i.e. Wi-Fi, WiMAX, LTE and TV White Spaces) and platforms (i.e. stratospheric balloons and unmanned aerial vehicles) to introduce broadband services in the Andes and Amazon regions of Peru. The technical analysis evaluates appropriate technical design and configuration settings for the wireless technology options and platforms by conducting propagation modelling and coverage simulation. The engineering cost analysis estimates deployment and operating costs of the access networks for each technology for a 10-year period. The technical and engineering cost analyses 
include scenarios based on both current and proposed telecom policies and regulations. In this manner, the technical implications and cost impact of current and proposed policy and regulations are analyzed in an objective quantitative manner.

This chapter presents, first, the broadband service demand estimation, which includes the selection of the study areas, defining the network coverage, and estimating customer and speed demand for a 10-year period. The second part is the technical analysis (wireless access network design and simulations), which uses the results from the broadband service demand estimation (selected study areas, defined coverage and estimated demand) as inputs along with RF equipment technical specifications, spectrum regulation, digital terrain data, and land use and land coverage (LULC) data. The output of the technical analysis includes: 1) detailed access network design elements (i.e., number of nodes, towers, APs or BSs, backhaul radiolinks, CPEs, and energy requirement.) for the 10-year period; 2) area propagation coverage and service availability (i.e., coverage at higher and lower broadband speeds and percentage of service availability in the coverage areas). The third part is the engineering cost analysis which estimates deployment and operating costs for the 10-year period based on the output from the technical analysis, cost data of the network elements (i.e. RF equipment, infrastructure, towers, energy system.) and operating expenses (i.e. personnel, energy bills, interconnection ). The output of this analysis includes: 1) total deployment and operating costs for a 10-year period, 2) net present value of the total cost, 3) cost 
per user per month, and 4) cost per Mbps per month. Each part of this section provides detailed information of the data (i.e. sources, collection, and organization), as well as information of the calculations, inputs, outputs, and variables.

Figure 5 shows the structure (inputs and outputs) of the technical and engineering cost analyses using a bottom up approach. The results and interpretation of the technical and engineering cost analyses are presented in the next chapter.

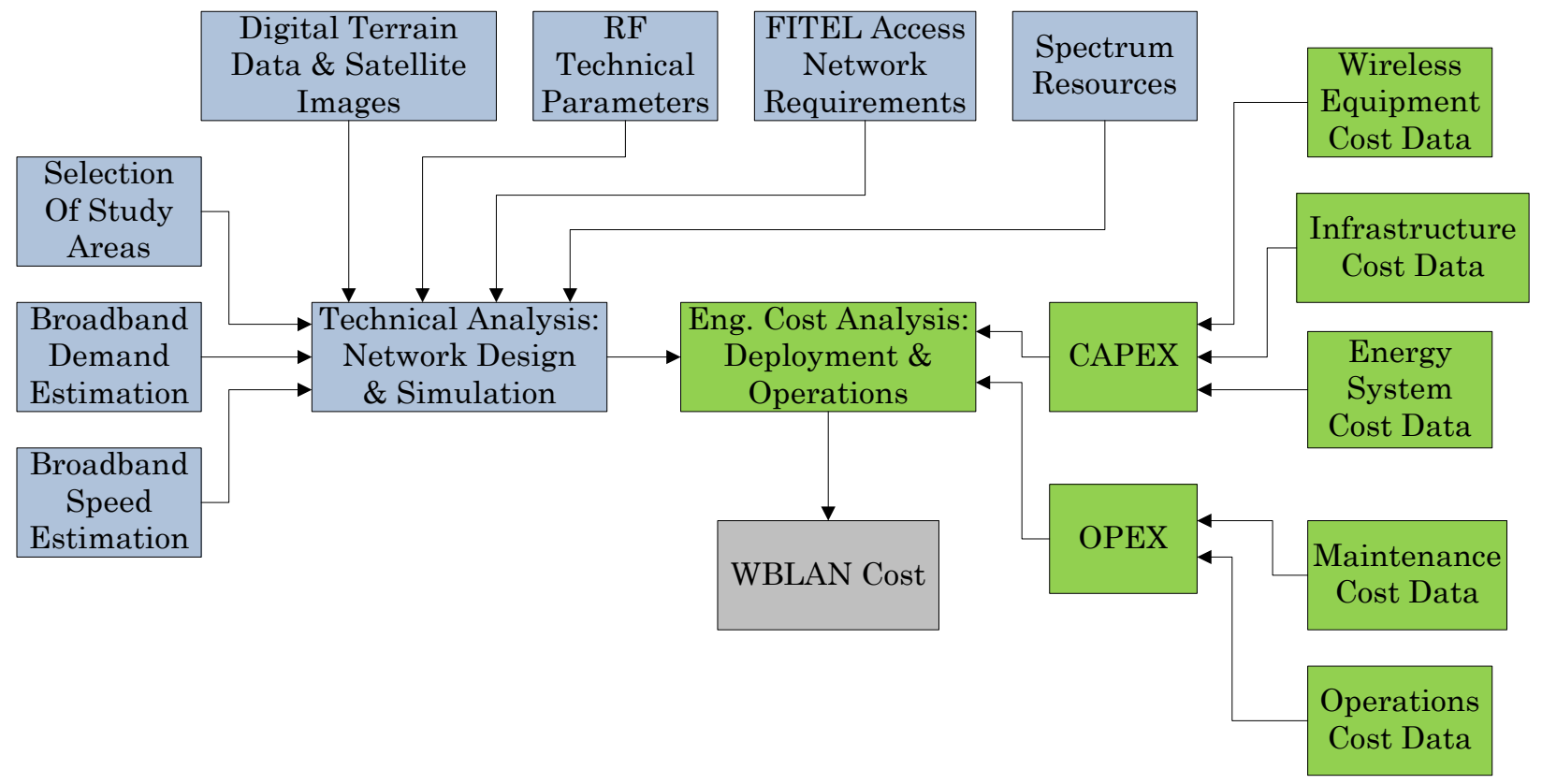

Figure 5. Structure of the technical and engineering cost analysis using a bottom up model 


\subsection{Broadband Service Demand Estimation}

\subsubsection{Selection of study areas and access network coverage Study areas in the Andes and Amazon regions}

This study focused on rural areas in the Andes (mountains) and Amazon (rainforest) regions due to their low broadband penetration and the interest of the Peruvian universal service agency FITEL to improve social and economic conditions in these areas of Peru as part of the National Broadband Plan. Two geographic settings were selected as case study areas, one in the Andes region and another one in the Amazon region. The selection criteria for the study areas targeted the following: 1) low broadband penetration areas, 2) rural remote locations, and 3) harsh landscape settings for analyzing wireless technology performance in extreme conditions. As part of the first four FITEL broadband bids titled "Broadband Deployment for Integrated Connectivity and Social Development" [84] [85] [86] [87], where broadband service providers compete for a subsidy for the deployment of broadband networks in four underserved states (Apurimac, Ayacucho, Huancavelica and Lambayeque), FITEL published the following documents in mid-2014: legal framework for the bid, contract template, feasibility study, technical specifications for the transport (optic fiber links) and access (RF links) networks, and geographical and population data of primary interest towns and customers. These data consisted of lists and geographical 
coordinates of towns and government institutions (mandatory customers) such as health posts, schools, police stations, and municipality buildings.

We reviewed the geographical and population data for each state (Apurimac, Ayacucho, Huancavelica and Lambayeque) in each of the bids available by mid2014. Residential Internet penetration statistics of 2013 indicated that the residential penetration was 5.3\% in Apurimac, 4.9\% in Ayacucho, 2.5\% in Huancavelica, and $20.7 \%$ in Lambayeque [88]. The state of Lambayeque had the highest broadband penetration due to its location in the coastal region and access to fiber optic backhauls; therefore, it was not included for further selection of the study areas. Of the other three states, Apurimac and Huancavelica are located in the central Andes; Ayacucho is also located in the central Andes but has a part of its territory located in the western Amazon region. Based on the low penetration criterion, the state of Huancavelica was chosen for the Andes region due to having the lowest penetration, and the State of Ayacucho was chosen for the Amazon region due to both its low penetration and partial location in this region. Then, potential study areas within these two states were evaluated using the criteria based on rural remote location and harsh landscape setting. It is important to highlight that we limited each study area size to one district, which included the district capital and towns within the district. This study area size was defined based on the FITEL bids which stated that each wireless access network will cover only one district and will begin in a district capital and expand into the district to provide service to mandatory towns selected by the government. The district 
capitals will have a point of interconnection to the optic fiber backbone allowing connecting the access networks to the Internet. The next step in the selection of the two study areas consisted in finding districts in Huancavelica and Ayacucho which met the criteria. To clarify how the Peruvian division of states into smaller geographical administrative units works: states are divided into provinces, and provinces are divided into districts. Depending on the context of an urban or rural area, a city or town in an urban area is divided into districts (large neighborhoods), or a district in a rural area consists of a group of towns (similar to counties in the United States). In this study, for purposes of simplicity, rural districts will be called counties and consist of a group of towns. Subsequently, proceeding with the study area selection, all rural district capitals (access networks heads and optic fiber interconnection points) were plotted in Google Earth. The remoteness of the district capitals was evaluated by measuring the distance and transportation conditions to other capitals. The harsh landscape setting was evaluated by analyzing the terrain, distance between towns in the district and line-of-sight from one town to another. Google Earth provided valuable tools for this selection process, such as geographical coordinates (latitude, longitude and altitude), location pins, distance measurements, path plotting and 3D-imagery, among others. Additional bulk conversion tools were used to convert large tables containing district capital and towns names and their coordinates into location pins in Google Earth digital satellite maps. 
Based on the criteria and process described above, the selected area in the Andes region was the Yauli county, in the state of Huancavelica in the central Andes of Peru, and the area in the Amazon region was the Samugari county, in the state of Ayacucho in the central Andes and western Amazon rainforest of Peru. The capital of the Yauli county is Yauli, located at 3,400 meters (11,155 feet) above sea level. The landscape in this county consists of mountains, hills and moderate or minimal vegetation. There are tens of agrarian towns located around the county capital. Most towns consist of a main square surrounded by houses and government buildings, and other houses spread in open land close to agricultural land. The capital of the Samugari county is Palmapampa, located at 810 meters (2,660 feet) above sea level. The landscape in this county consists of hills and dense vegetation (rainforest jungle). There are agrarian towns in the county mostly located along the Apurimac River. In this area, most towns consist of a main square surrounded by houses and government buildings with only a small percentage of houses in open land due to the dense jungle vegetation. Figure 6 shows the Yauli and Palmapampa towns in the two selected study areas. 


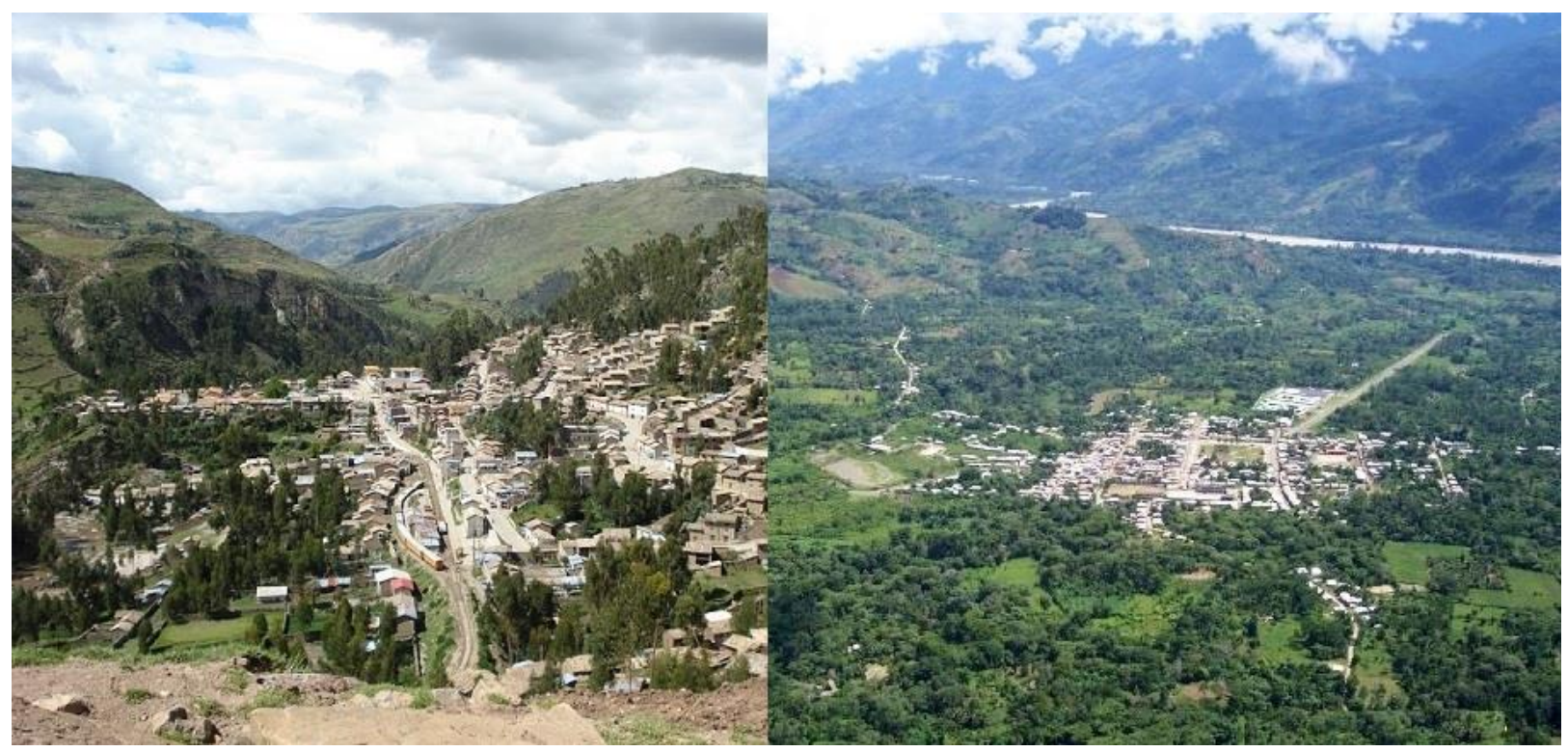

Figure 6. Yauli town, capital of the Yauli county in the state of Huancavelica in the central Andes of Peru (Left). Palmapampa, capital of the Samugari county in the state of Ayacucho in the central Andes and western Amazon of Peru (Right).

\section{Wireless access network coverage in the study areas}

After selecting the two study areas, the next step was to define the geographical coverage for the fixed broadband service. It was necessary to establish criteria to select which towns, among tens of towns in each county, will be part of the access network coverage. The initial criteria followed FITEL's bids, which included towns having population of at least 300 people (before network deployment), student population of more than 100, or having at least one health post, one police station or any other government institution [85] [86]. Additionally, towns that meet these criteria must not have any available broadband service or have a low quality service, and must have public electricity grid service. Then, towns meeting these criteria were included in the network coverage, and within these towns, mandatory customers were government institutions such as 
elementary/high schools, health posts, police stations, etc. These mandatory customers will be the anchor customers for the broadband service provider for the 10 years period. Based on the FITEL bids' criteria, the coverage in the Yauli county included 16 towns, 22 schools, 11 health posts, 1 police station and 2 other government buildings. In the Samugari county, the coverage included 19 towns, 28 schools and 5 health posts. The number of mandatory FITEL bids' towns remained static for the deployment and following 10 years, while the number of government clients slightly increases around 10\%. Regarding residential customers, the FITEL bids' left up to broadband service providers to estimate and plan for this demand. In addition to FITEL's criteria, this study also included towns that might become potential attractive markets for the fixed broadband service in the next 10-years. Based on these additional criteria, the access network coverage in both counties also included towns which will reach at least 300 people within the next 10 years after deployment, or which currently have broadband service, as the new wireless broadband network can bring another competitor to the market for the benefit of customers.

Combining the FITEL and our additional service coverage criteria, the total fixed broadband service coverage for the two study areas is as follows:

- Yauli county: 27 towns (shown in Figure 7) with a total estimated population of 17,338 and 3,580 households in 2015 , covering a 62.71 square mile area. 
- Samugari county: 29 towns (shown in Figure 8) with a total estimated population of 16,909 and 4,196 households in 2015 , covering a 662.52 square mile area.

The service coverage in both study areas includes government (anchor) and residential customers. The next subsection estimated the residential customer and speed demands.

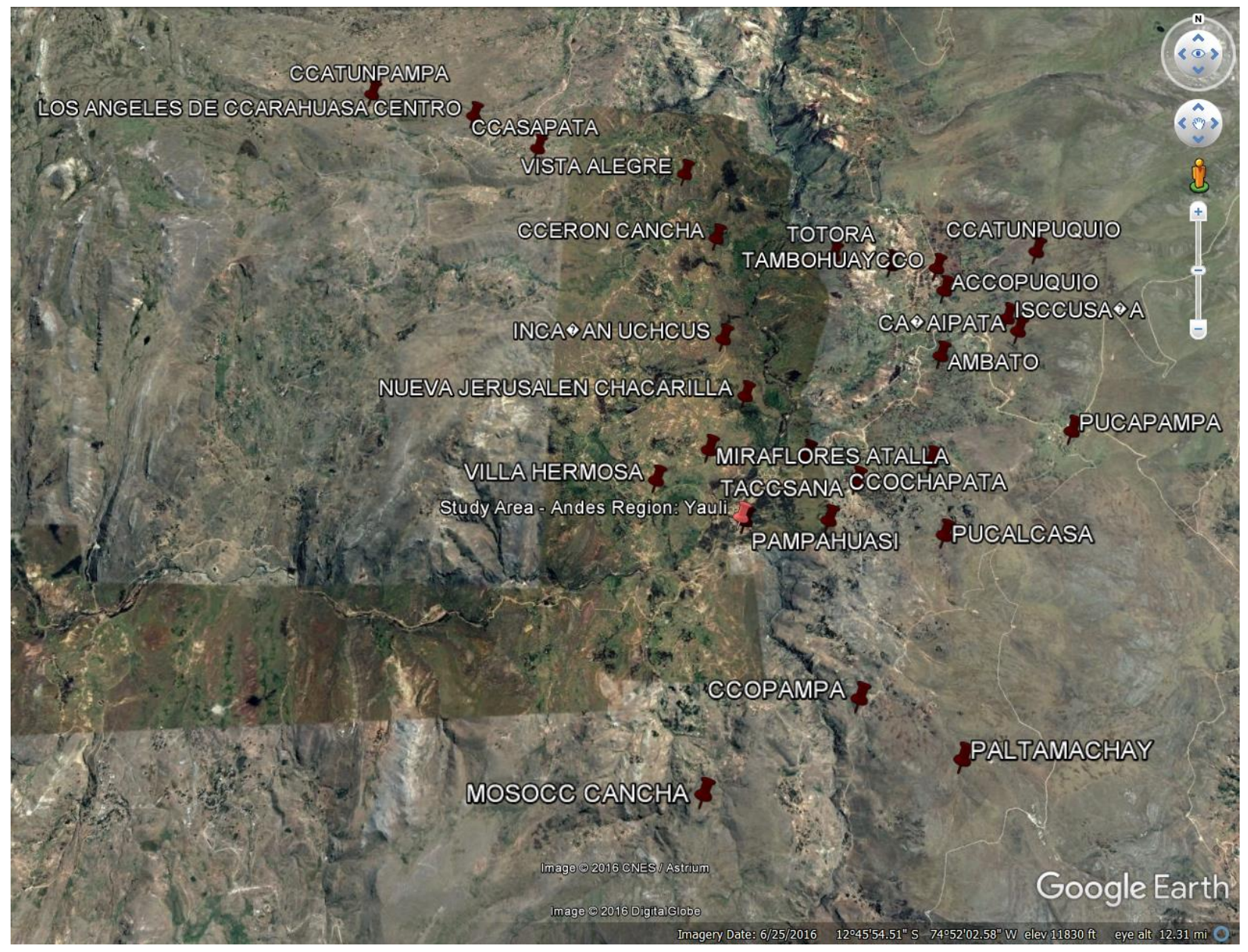

Figure 7. Google Earth view of the 27 selected towns in the Yauli county study area in the Andes Region. 


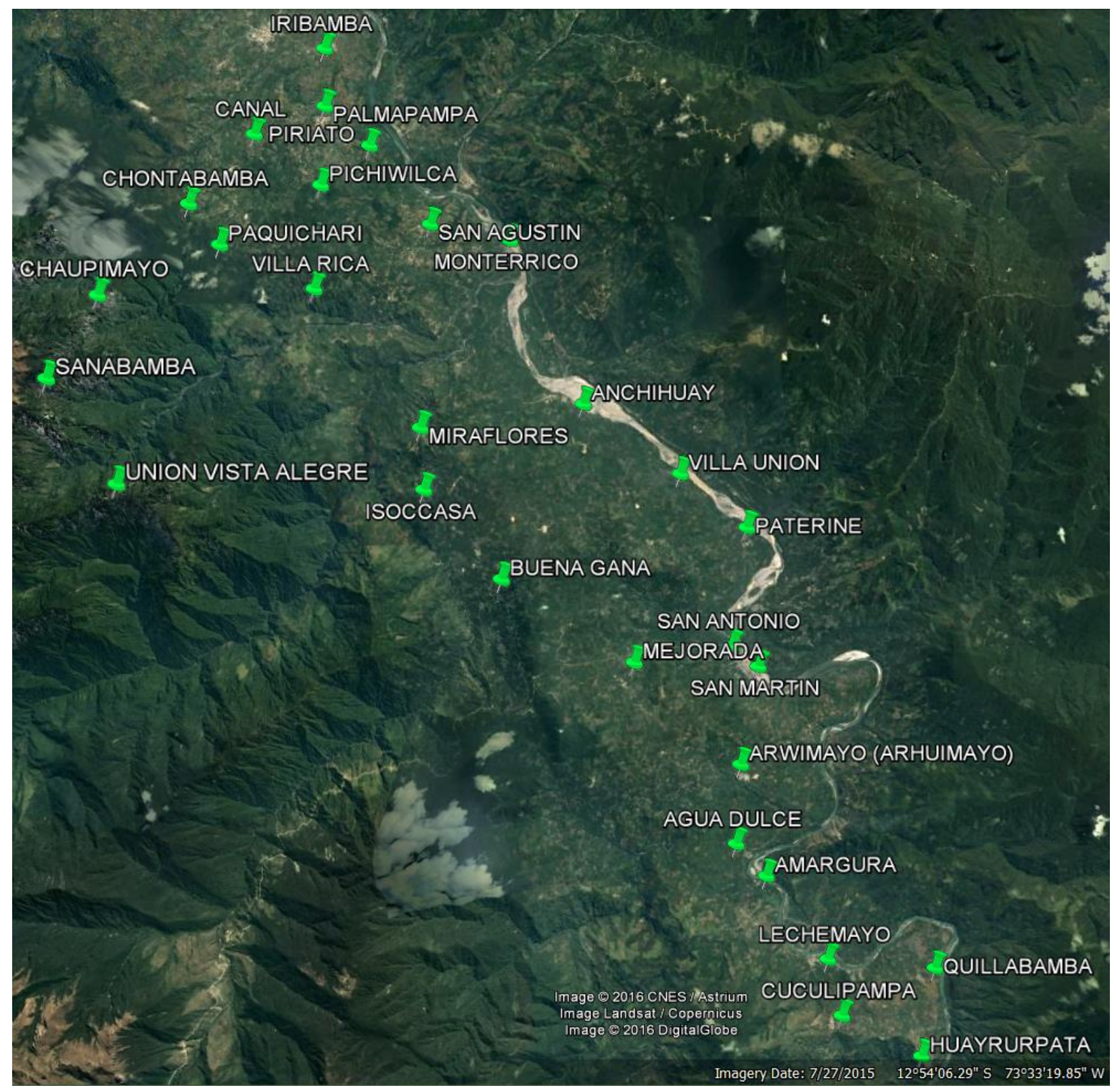

Figure 8. Google earth view of the 29 selected towns in the Samugari county study area in the Amazon Region. 


\subsubsection{Broadband residential customer and speed demand estimation for a 10-year period}

\section{Broadband customer demand}

The FITEL bids defined the demand for mandatory or government customers and included lists of these customers for each state, such as schools, health posts, police stations, and municipalities, among other government buildings. It was up to broadband service providers to estimate the residential broadband demand at deployment and for the next 10 years. Then, the next step in the study was to estimate this demand in the states of Huancavelica and Ayacucho, where the two study areas are located. For this estimation, data included residential Internet service penetration [88], fixed monthly Internet service price [89] and average monthly income from the 24 states and three regions (Coastal, Andes and Amazon) of Peru [90]. These data are available in the official websites of the following Peruvian government agencies: National Institute for Statistics and Information (INEI), Telecommunications Private Investment Supervision Agency (OSIPTEL), Transport and Communication Department (MTC).

Residential Internet service penetration data was used as a proxy for residential broadband service penetration. This residential Internet service penetration included customers using both narrowband (dial-up) and broadband; however, after 2004, there was a decreasing trend of narrowband customers and, by 2012 , narrowband penetration was only $0.3 \%$ of the total residential Internet penetration [88]. Based on this residential Internet service penetration data (from 
2002 to 2014), in 2014, the state of Ayacucho had a 6.2\% penetration (0.7\% average annual growth in the last five years), and the state of Huancavelica had a $2 \%$ penetration ( $0.3 \%$ average annual growth in the last five years). This historical penetration described the status quo penetration growth in these underserved states, and did not include yet the penetration impact of broadband services provided by new wireless access networks at a subsidized rate under the National Broadband Plan.

After the deployment and during the operation of new wireless broadband access networks in the two study areas, it is expected an increase in the average annual demand growth (from $0.7 \%$ and $0.3 \%$ ) mainly due to two factors: 1 ) new availability of broadband service in previously underserved or unserved areas, and 2) a low initial Internet service price set by the government that includes a monthly rate subsidy. To estimate the increased annual demand growth, first, we analyzed the correlation between the monthly Internet service penetration, average monthly income and fixed Internet service penetration for all states of Peru in 2014. A correlation of $\mathrm{R}^{2}=0.7764$ was calculated between the percentage ratio of the monthly Internet service price to the average monthly income and the fixed Internet service penetration. Based on this correlation, a residential fixed Internet service demand curve was generated, and is shown in Figure 9, having in the $\mathrm{x}$-axis the percentage of the monthly Internet service price out of the monthly average income, and, in the y-axis, the fixed Internet service penetration for all states of Peru in 2014. 


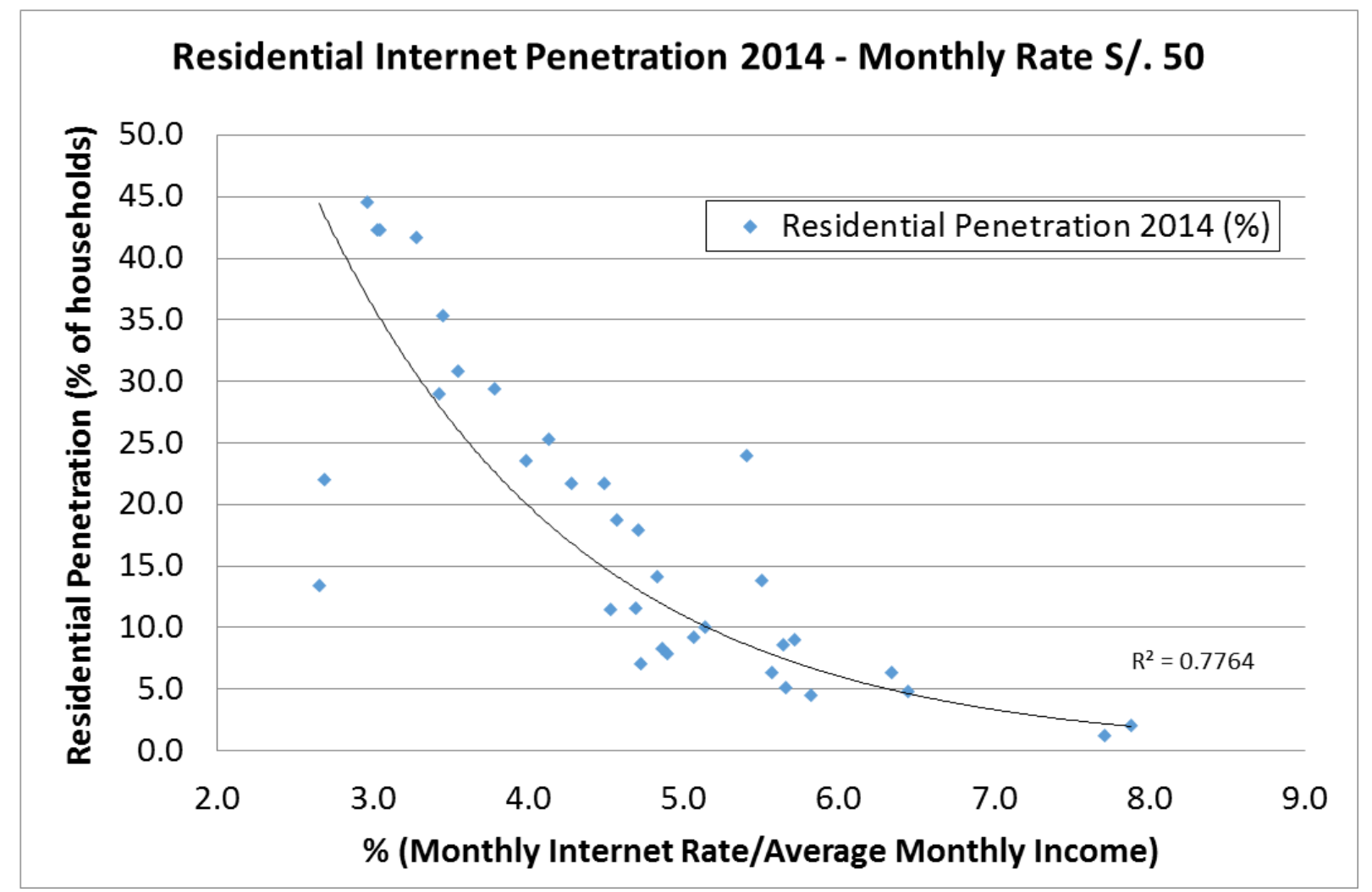

Figure 9. The Internet service price as percentage of monthly income determines the Internet service penetration or Internet demand curve

The next step was to use the FITEL's proposed regulated Internet price (PER 37 or USD 12) [85] [86] in the states of Huancavelica and Ayacucho to calculate the shift of the penetration for these states in the demand curve due to the updated percentage of monthly Internet price out of average monthly income. Based on the proposed regulated Internet price, the updated ratio of Monthly Internet Rate/Average Monthly Income for Ayacucho and Huancavelica are 4.41\% and $5.58 \%$, respectively. These percentages were introduced in the demand curve in order to identify states that are already operating in those conditions, which were the states of Tumbes and Lima (excluding the city of Lima which has a much 
higher yearly growth). Next, it was estimated that Huancavelica and Ayacucho's Internet penetration will follow in the coming years the Internet penetration and annual growth of the states of Tumbes and Lima. In Ayacucho, the fixed residential broadband penetration (as percentage of households) for 2015 (year 1 in this study) will be $9.1 \%$, and in Huancavelica will be $4.7 \%$, with an annual average growth of $2.9 \%$ and $2.7 \%$, respectively. This customer demand penetration and annual growth will be applied to all towns in the study areas except for the capitals. For the case of the towns of Yauli and Palmapampa (capitals of the selected counties), it was assumed that each broadband service provider (an ADSL incumbent and the new broadband provider) will have a $50 \%$ of the market after deployment and the ensuing next 10 years.

After we completed the demand estimation for the two study areas, FITEL shared their demand estimation for the state of Ayacucho based on a complex demand modeling from a consulting report by the Inter-American Development Bank which used detailed econometric models and coefficients for technology adoption curves [85]. For a 10-year period, FITEL's report estimated a broadband penetration (of total households) of $4.6 \%$ for year 1 and $35.7 \%$ for year 10 , and our estimation was $9.1 \%$ for year 1 and $35.2 \%$ for year 10 . Considering that both customer demand estimations were carried out using different approaches, the estimations are close to each other. It is important to highlight that both estimations were conducted at state-wide level due to the lack of disaggregated data at province or county (district) levels. 
Finally, for the fixed residential broadband customer demand estimation as a percentage of total households, the total households also includes an annual growth rate based on historical population and household growth in the last decade in the two study areas (Yauli and Samugari counties).

\section{Broadband speed demand}

This study also includes an estimation of broadband speed demand for the 10-year period. FITEL bids set an initial rate of $2 \mathrm{Mbps}$ for government and residential customers, and leave up to the broadband service provider to plan for broadband speed growth. The broadband speed estimation used data provided by Internet service operators to the regulator OSIPTEL such as historical (2001-2015) residential Internet penetration by technology (ADSL, cable, and wireless), type of service (residential, business and Internet cafe) and speed (64kbps, 128kbps, $256 \mathrm{kbps}, 512 \mathrm{kbps}, 1024 \mathrm{kbps}, 2048 \mathrm{kbps}, 4 \mathrm{Mbps}, 8 \mathrm{Mbps}$ and $>16 \mathrm{Mbps})$ [91]. However, this data was based on operator's aggregated nation-wide residential customers and not by state or geographical location. Then, the next step was to break down this data into categories, where one category can be used as a proxy to estimate the potential speed growth demand in rural areas in Peru. In 2014, the city of Lima had an estimated population of 8,751,741 (28.4\% of total Peru's population) and 2,141,394 households (27.8\% of total Peru's households) [92]. Lima is also the largest and fastest growing market for fixed residential broadband services, accounting for $50 \%$ of the total nation-wide customers and having access 
to international and domestic optic fiber backbones. Based on these population and market statistics, this study assumes that Lima's speed demand growth would not be representative of suburban and rural areas in Peru. Then, the aggregated nation-wide data was broken down in two geographical areas or categories: 1) Lima, and 2) rest of the country including urban, suburban and rural areas. The second category will be used to estimate the fixed residential broadband speed growth rate for the two study areas. Using data of fixed residential broadband penetration (as percentage of total households) and the total households in Lima, and more importantly, assuming that the highest speed residential customers were located in Lima, it was calculated the disaggregated "rest of the country" broadband speed demand from 2005 to 2015 . This calculation basically assigned the highest speeds to Lima until reaching Lima's residential customer demand and the rest of users and speeds were assigned to the second category.

The result of the residential broadband speed calculation for the "rest of the country" category, based on fixed residential Internet penetration data by speed and by technology, showed that the average nation-wide Internet penetration speed (for all combined technologies) from 2005 to 2015 increased from 140kbps to $732 \mathrm{kbps}$, with a compound average growth rate (CAGR) of $18 \%$. The average nation-wide Internet penetration speed for the "rest of the country category" was calculated by multiplying each broadband speed by the number of fixed residential customers at each speed and dividing them by the total customers. Then, based on this historical residential broadband speed CAGR of $18 \%$, this study will round up 
and use a residential broadband speed demand CAGR of $20 \%$ for the two study areas. Figure 10 shows the estimated residential broadband speed demand from year 1 (2015) to year 10 (2024) with an initial speed of 2Mbps and CAGR of 20\%. The final average fixed residential speed demand will be quantified using the following commercially available speeds: 2Mbps (Y1-Y3), 4Mbps (Y4-Y7), 8Mbps (Y8-Y9) and 12Mbps (Y10).

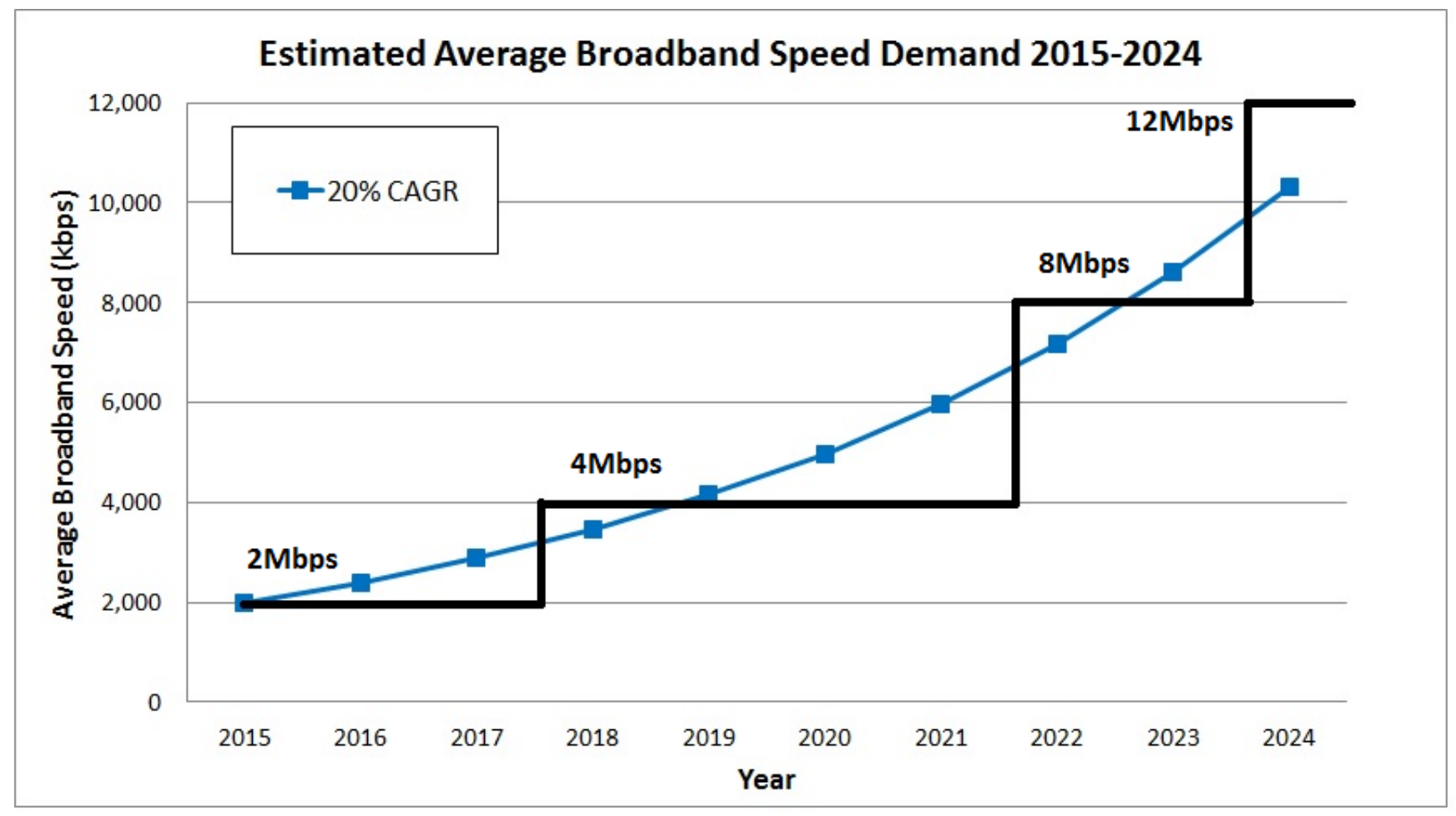

Figure 10. Estimated broadband speed demand 2015-2024 for rural areas in Peru

\subsection{Technical analysis: wireless broadband access networks design and simulation in EDX}

This section describes the technical analysis which evaluates efficient stateof-the-art broadband access network design and configuration settings for the wireless technology options (Wi-Fi, WiMAX, LTE and TVWS) and platforms 
(aircrafts and super-pressure balloons) by conducting propagation modelling and coverage simulations. The EDX Signal Pro software is used for the network design and propagation analysis. The access network scenarios include both terrestrial and stratospheric wireless access networks.

The technical analysis uses the access network coverage and the residential broadband customer and speed demand for a 10-year period in the two study areas described in the previous section. This analysis also uses digital terrain data, and land use and land coverage (LULC) data, RF equipment technical specifications, FITEL bids' access network requirements, and current spectrum regulation, among others. The results of the technical analysis, which will also be used for the engineering cost analysis, includes a detailed list of access network design elements (i.e. number of nodes, towers, APs or BSs, backhaul radiolinks, CPEs, energy requirement.) for a 10-year period. Other results include EDX simulation results such as area coverage at different broadband speeds (which depends on modulation and coding schemes for the different wireless technologies) and percentage of service availability in the coverage areas.

\subsubsection{Propagation model for simulations}

The EDX Signal Pro software was used for the wireless access network design and propagation modeling. EDX allows using different propagation models such as the free space, empirical, stochastic, and physical models. Empirical models are based on measurements and provide a first order estimate for locations similar to those where the measurements were conducted. Correction terms can be 
added to these models to adapt them to different environments and frequency ranges. Some empirical models are widely accepted for cellular communications (e.g., Extended Hata or Cost 231-Hata). Stochastic models are derived from empirical models and predict attenuation based on some properties which describe the study environment such as average building height and street width (e.g., Cost 231 Walfisch-Ikegami) [93]. Physical models are based on the free space path loss equation and use terrain data, land use and land coverage (LULC) data and building data to predict the propagation of the signal. These physical models are an appropriate fit for site-specific modeling due to using specific environmental data for specific study areas. The Anderson-2D is a physical model which uses traditional ray techniques to calculate path loss. Depending on the transmitter receiver conditions, the model takes one of two approaches, either line-of-sight or obstructed paths calculation. For the line of sight calculation, the model uses free space loss calculation for the direct ray between transmitter and receiver with additional loss factors for the case of slightly obstructed first Fresnel zone, and also uses ground reflection including specular reflection coefficients to determine the amplitude and phase of reflected rays. For the obstructed calculation, the model calculates the diffraction attenuation using the Epstein-Peterson approach extended to multiple knife edges [94].

For the propagation modeling, Anderson-2D propagation model was selected due to the following [93]: 1) supports the use of terrain, land use and land coverage (LULC) and building data, 2) this model was selected by the Telecommunications 
Industry Association (TIA) as the definitive model for TSB-88A/B for predicting coverage and interference for mobile radio systems in the United States, and 3) the model was found to outperform (based on the average standard deviation of the prediction error) the Longley-Rice (ITM), TIREM and other physical and empirical models when compared against measurements.

\subsubsection{Environment simulation settings for the Andes and Amazon study areas}

For the access network design and propagation analysis in EDX, digital terrain data and land use and land coverage (LULC) data were used as environment simulation setting specific for the two study areas in the Andes and Amazon regions. Using these data allows accurate propagation calculations between the base stations or access points and the customer premise equipment (CPE), which includes the effects of the terrain and different types of land use and land coverage in the propagation losses. As a result, the propagation analysis will provide access network coverage results closer to an actual network deployment.

Digital terrain data from the Advanced Spaceborne Thermal Emission and Reflection Radiometer (ASTER) Global Digital Elevation Model Version 2 (GDEM V2) was used to set the terrain for the simulations in the two study areas. The ASTER GDEM V2 digital terrain data was released in 2011 by the United States National Aeronautics and Space Administration (NASA) and the Ministry of Economy, Trade, and Industry (METI) of Japan [95]. This digital terrain data provides 30-meter resolution global maps. Figure 11 shows the digital terrain data 
for the Yauli county in the Andes Region study area which can be visualized in EDX as shaded relief, elevation contours, among other formats.

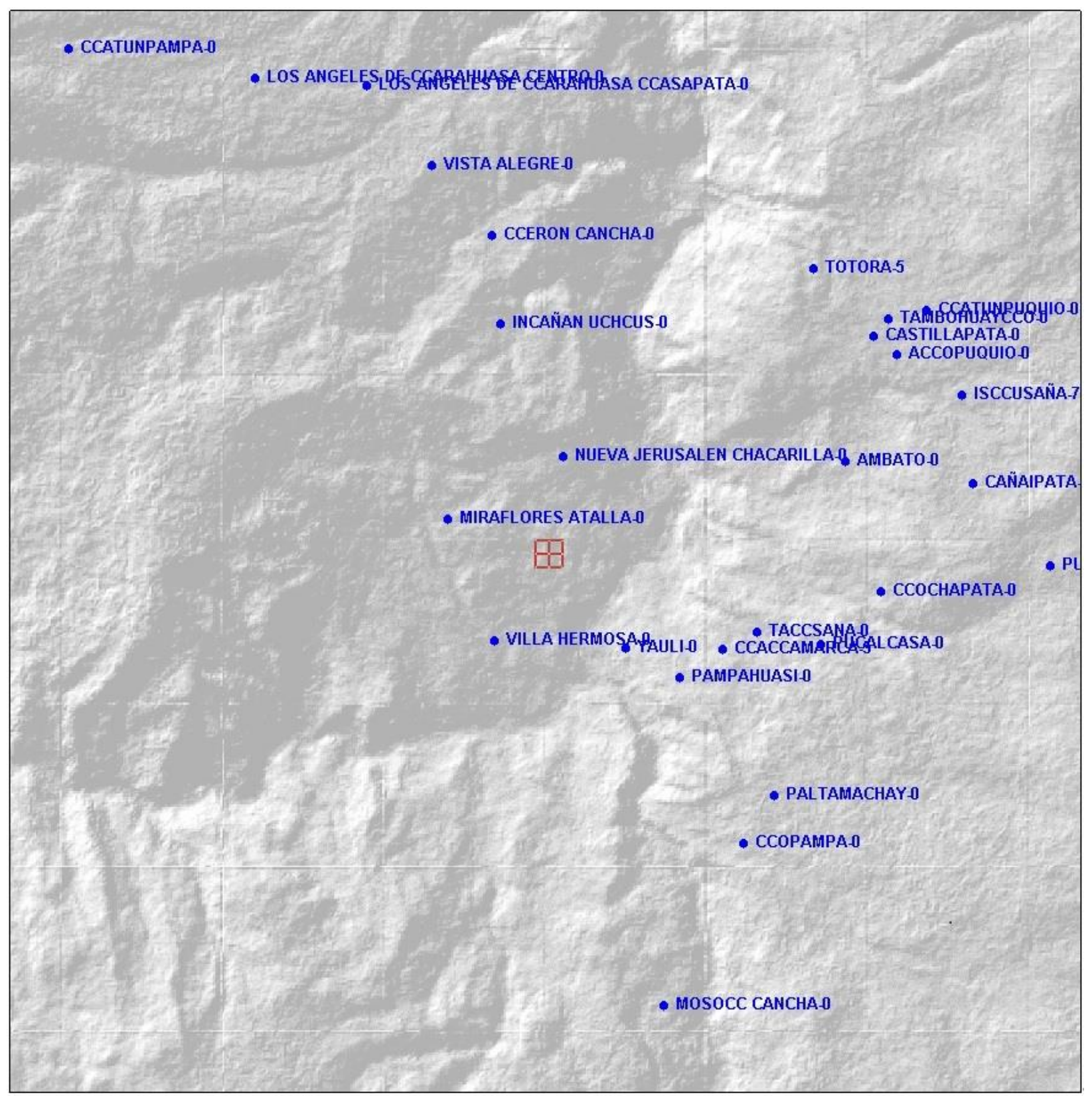

Figure 11. ASTER GDEM V2 terrain data for the Yauli county area in the Andes Region. Terrain data presented as shaded relief.

For setting the LULC in the two study areas, digital satellite images from Google Earth and Bing were used to identify different terrain use and coverage. 
For example, towns or residential areas, agricultural land, vegetation, forest, and rivers or other water bodies, among others. After identifying the terrain use and coverage in the two study areas, it was necessary to plot these areas in EDX. EDX will include additional losses generated by the different terrain use and coverage in the propagation calculations and simulations. EDX uses specific losses or attenuation coefficients associated to different terrain use and coverage from the Telecommunications Industry Association's TSB-88.1 C document, which typifies losses based on 10 clutter categories, to determine the appropriate attenuation value for the different terrain areas [96]. In the Yauli county area, the following LULC areas and heights were identified and plotted in EDX: forest (15 meters), grassland (1 meter) and towns (4 meters). In the Samugari county area, the following LULC areas and heights were identified and plotted in EDX: rainforest (15 meters) and towns (4 meters). Figure 12 shows parts of the Yauli and Samugari areas and the plotted terrain use and coverage or LULC areas in different colors. As shown in this figure, EDX allows keeping the digital satellite image in the background and plot different LULC areas in another layer on top. 


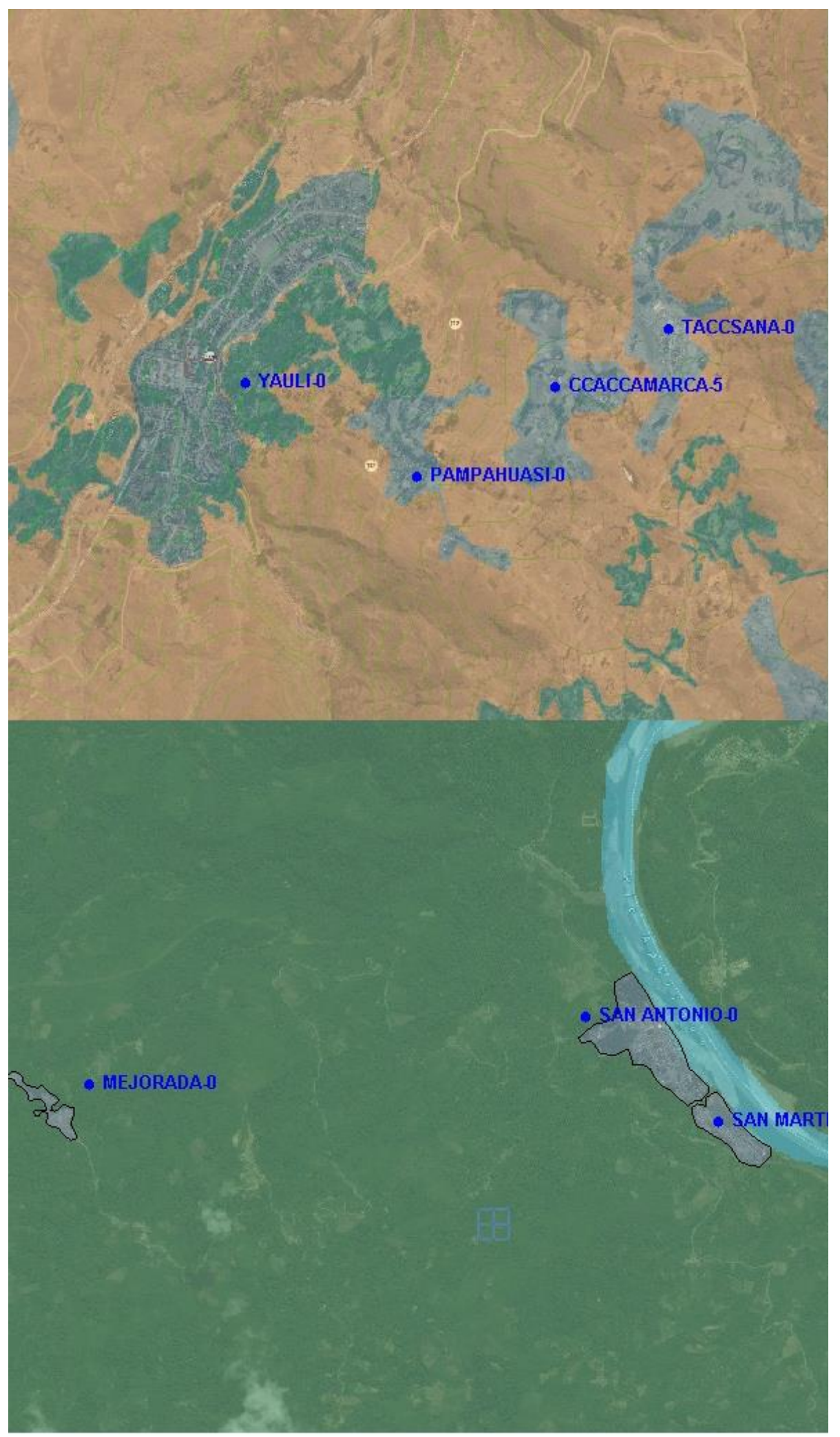

Figure 12. LULC areas. (Top) Yauli towns in gray, grassland in orange, and forest in green. (Bottom) Samugari towns in gray, forest in green and river in blue. 


\subsubsection{RF equipment and network simulation settings}

The next step after setting the propagation model and environment data (digital terrain data and LULC data) for the simulations in EDX was to set the technical specifications or parameters of the RF network equipment (base stations, access points and customer premise equipment). These parameters include frequency, transmission power, antenna gain, antenna diversity, antenna radiation pattern, cable losses, and receiver sensitivity, among others. The technical parameters and specifications were obtained from datasheets and manuals available on vendor websites or made available from vendor representatives.

Section 3.3 Engineering Cost Analysis describes the technical specifications gathering process, as it is related to the collection of cost data by requesting $\mathrm{RF}$ equipment quotations from vendors. For the analyzed technologies (Wi-Fi, WiMAX, TVWS and LTE), equipment from several options in the market was reviewed and selected. The methodology used to benchmark the different RF equipment options (BS, AP and CPE) used the key performance indicators (KPI) defined in [97] such as maximum range and throughput (output power, sensitivity, antenna gain, and data rates), next generation capabilities (clients load, high speed ports), quality of service, market status (market availability), innovation potential, and upfront and operating expenses of the equipment. Figure 13 shows EDX setting windows which allows entering and configuring technical specifications of access points or base stations, including transmission and receiving parameters and antenna radiation patterns. 

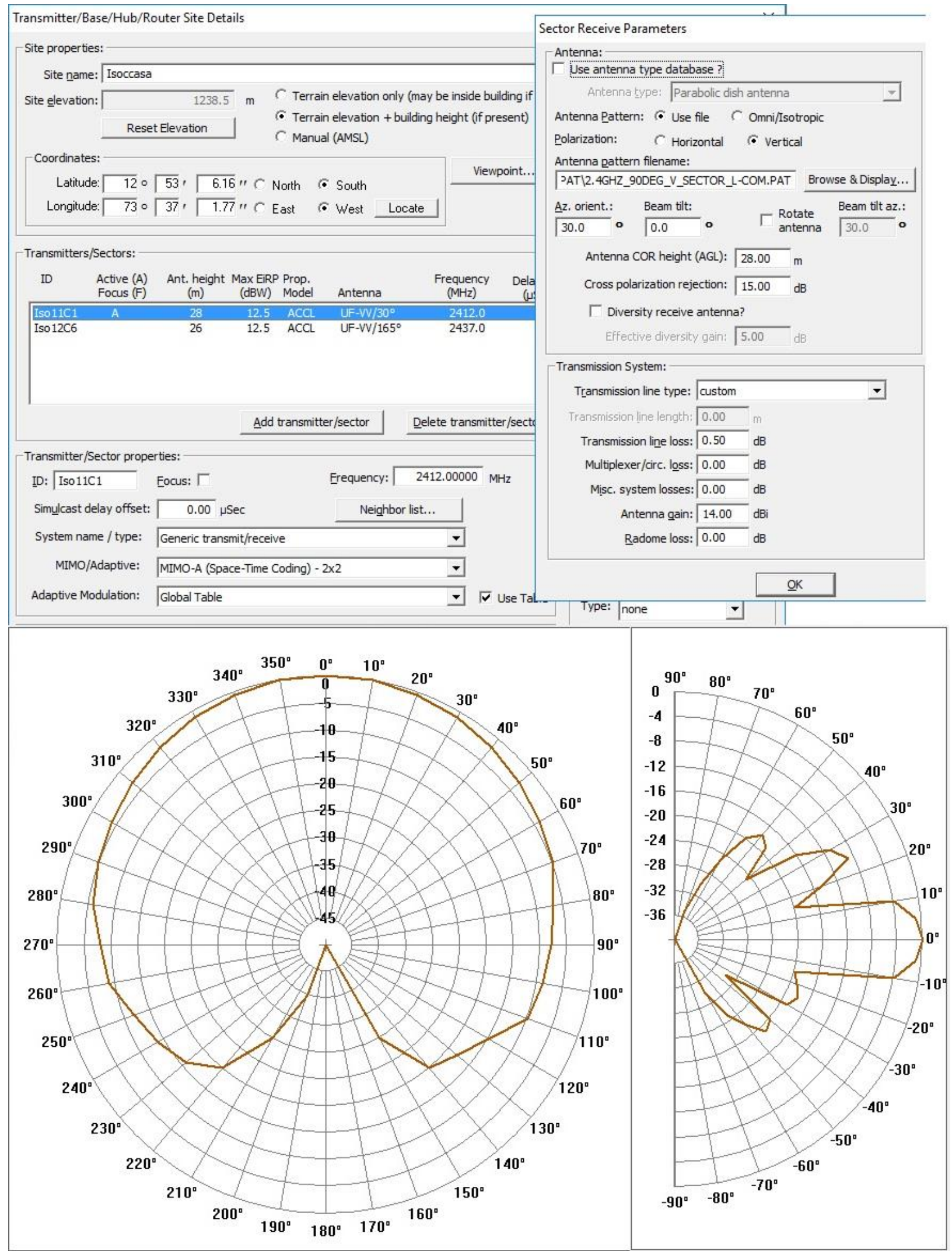

Figure 13. RF parameter settings in EDX for a base station or access point. 


\subsubsection{FITEL access network design requirements}

Another important input for the access network design process and the engineering cost model is the FITEL bids' access network design requirements, which range from potential wireless technologies, frequencies, and network service availability to energy system technical specifications, characteristics of the nodes and construction requirements. These requirements will play an important role when designing the network due to their potential impact on the network topology and configuration. At the same time, energy, towers and civil engineering requirements will have impact on the deployment and operating costs of the network. This subsection presents a list of FITEL's main access network requirements which were incorporated in the technical and engineering cost analyses [85] [86]:

- Wireless technology: The FITEL projects allow broadband service providers to use any wireless technology as long as spectrum bands are available to them. The wireless equipment must also comply with ITU-R and ITU-T technical recommendations.

- Network service availability: The availability of the access network must be at least $98 \%$ per year.

- Network capacity and broadband speed: The network must be able to handle customer demand in the mandatory towns providing a minimum download speed of $2048 \mathrm{kbps}$ and 512kbps upload speed per mandatory (government) customers with a $40 \%$ guaranteed speed at peak hour. The 
guaranteed speed for secondary (residential) customers is to be determined by the operator in agreement with the telecom regulator. The network must be able to handle customer demand growth.

- Type of nodes: The access network must consist of the following types:

- District Node: Interconnects the access network with the transport network (optic fiber backbone) and can have an access network point of presence (POP).

○ Intermediate Node: Retransmits signal from a District Node to a terminal Node, and can have an access network POP.

○ Terminal Node: Operates as an access network POP.

- Nodes location: There must be at least one access node in each of the mandatory towns. In case that selected towns, due to their proximity to each other, can be served by only one node (district, intermediate or Terminal), the operator can use this exception, as long as the towns are not farther than a 2000 meter distance.

- Energy system: District nodes must have at least one diesel power generator, rectifier charger, control/transfer board, peak voltage breaker and batteries, and grounding system. Intermediate and terminal nodes must have at least a rectifier charger, control/transfer board, peak voltage breaker and batteries, and grounding system. The operator must provide energy to the network nodes in towns which lack public grid power. 
- Civil engineering and node construction: The FITEL bids also have specific civil engineering and construction requirements regarding access security system, perimeter wall and fence. Additionally, the node specifications include the area of the equipment room and size of the shelter, among others. Inside the node, the telecommunications equipment must be kept year round in the following room conditions: temperature $\left(16^{\circ} \mathrm{C}-24^{\circ} \mathrm{C}\right)$ and humidity (40\%-90\%).

- Towers: The towers for the access network must be self-supported and with 12 meters minimal height. The height of the tower must allow establishing backhaul and access radio links.

- Interconnection: Customers of the access network must be able to connect customers of other broadband service providers, so the operator must carry out interconnection agreements and contracts with other broadband operators.

The following FITEL bids requirements were excluded from the technical and the engineering cost analysis due to falling outside of the scope and delimitations of this study:

- Computer work stations for government customers (schools, health posts, police stations, etc.).

- Generation of Intranet service and content.

- Social awareness campaign and information technology training for customers 
- Installation and operation of a Network Operating Center for managing all access networks in one state.

- Implementation of a customer service and marketing center.

\subsubsection{Spectrum resources, reuse factor and overbooking}

The design of the wireless broadband access network also incorporated current Peruvian spectrum regulation covered in Section 2.4.2 Spectrum Regulation. Defining the amount of spectrum resources for each wireless technology based on current regulation was critical for the access network design process. The available spectrum resources, overbooking ratio and reuse factor will determine the maximum wireless broadband capacity that each node of the network will be able provide before requiring additional nodes deployments. Furthermore, these additional deployments would require reducing the coverage of each node (or reducing the cell size) in order to accommodate all nodes without generating spectrum interference issues. This study uses the following spectrum resources for the wireless technology options:

- Wi-Fi: $2.4 \mathrm{GHz}$ using 3 non-interference channels of $20 \mathrm{MHz}$.

- WiMAX: $3.5 \mathrm{GHz}$ using 5 channels of $10 \mathrm{MHz}$ (spectrum cap of $50 \mathrm{MHz}$ per operator).

- LTE: $1.7 / 2.1 \mathrm{GHz}$ bands using 1 channel of $20+20 \mathrm{MHz}$ (spectrum cap of $20+20 \mathrm{MHz}$ FDD per operator). For LTE, the design follows current LTE network deployment practices of reusing the same spectrum (same channel) 
in a node with 3 sectors. The LTE technology allows for this type of deployment and reuse. For the proposed spectrum and policy scenarios, the technical and cost implications of using 2 LTE channels of $20+20 \mathrm{MHz}$ were also analyzed.

- TVWS: Digital broadcast television frequencies range from $470-608 \mathrm{MHz}$ to $614-698 \mathrm{MHz}$ bands; a total of 37 digital television channels (each channel is $6 \mathrm{MHz}$ ). There is currently no regulation regarding the use of white spaces in Peru. Since in rural areas there are only a few broadcast television operators or re-transmitters using less than 5 or 10 channels, this analysis assumes that the regulator would allow using up to 15 channels out of the 37 available channels.

The network design and coverage analysis used a re-use factor of 1 . In the two study areas, moderate propagation conditions due to terrain (hills and mountains) and terrain coverage (towns and dense vegetation) allow using the same channels in contiguous nodes with minimal risk of generating co-channel interference. This interference potentially affects CPEs receiving signal from two different nodes using the same channel. However, this potential interference can be reduced by using directive antenna radiation pattern for the CPEs (as used in this study for the network design), which focusses signal transmission and reception only towards the desired access point or base station.

The current overbooking ratio, or contention ratio, for rural areas in Peru is 1:10. This ratio was set by the regulator based on a concurrence factor (percentage 
of users accessing to the service at the same time) at 0.25 or $25 \%$ in rural areas, and a minimum guaranteed speed of 0.4 or $40 \%$ of the nominal (advertised) speed. The overbooking ratio is then calculated as following:

Overbooking Ratio $=$ Concurrence Factor $x$ Minimum Speed $=0.25 \times 0.4=0.1$

For the proposed spectrum and policy scenarios, it was analyzed the technical and cost implications of using an overbooking ratio of 10:1.

\subsubsection{Wireless access network design and simulation in EDX}

This subsection describes the wireless access network design process and the propagation and coverage simulations of the designed networks in EDX. The wireless access network design uses the elements presented in the previous subsections as design criteria and input, which include the network coverage, residential broadband customer and speed demand, digital terrain data, land use and land coverage (LULC) data, RF equipment technical specifications, FITEL bids' access network requirements, and current spectrum regulation, among others. Additional design criteria include designing an efficient state-of-the-art wireless access networks meeting speed and coverage requirements at the lowest cost (deployment and operation for a 10-year period).

\section{Wireless access network topology}

The topology of the wireless access networks for the analyzed technologies consisted of wireless backhaul radio links (point to point) and wireless access radio links (outdoor access points or base stations) placed in strategic locations both to 
achieve an optimal number of backhaul radio links and a minimum number of access radio links at the lowest cost. In both study areas, the access networks began in the district capitals (Yauli and Palmapampa), where the fiber optic backbone network will have points of presence (POPs). From the district capitals, the access network will expand over the study areas (through backhaul radio links and access base stations) to provide coverage to mandatory (schools, health posts, police stations, etc.) and secondary clients (residential and business) located in the chosen towns. The design process tested different locations for the network nodes and run EDX propagation and coverage simulations to evaluate such sites. In this manner, the design of the access network for each technology underwent several design iterations and EDX simulations to optimize the configuration and topology of the network to achieve the lowest cost.

\section{EDX simulations and area studies}

The EDX software provides several options to conduct propagation and coverage analysis. This study conducts the following simulations and area studies for the network design process:

\section{- Areas with signal levels above both base and remote thresholds:}

EDX calculates both areas where the signal received by the remote unit (CPE) is above the remote receiver threshold, and areas from where the signal received by the base station (or access points) from the remote unit is above the base threshold [98]. This study area was chosen due to its capability of providing combined coverage results for the downlink and 
uplink. Additionally, the simulation was set to show this coverage for the different data rates that wireless technologies offer. For example, higher data rates using 64QAM are shown areas closer to the AP/BSs, and lower data rates using QPSK are shown in larger areas farther from the AP/BSs. The results of these simulations are used to estimate the average bandwidth capacity per base station based on the coverage for the different speed rates.

- Number of servers at remote: EDX calculates the total number of transmitters that provide a signal above the receiver's thresholds at each point of the study grid. This area study is used to determine areas where more than one base station has coverage and there might be potential interference. As mentioned earlier, using a frequency re-use factor of 1 might lead to potential co-channel interference in areas where coverage of two or more AP/BSs overlap. This study helps to identity potential cochannel interference areas [98].

- $\mathrm{C} /(\mathrm{I}+\mathrm{N})$ at remote for strongest desired base station: EDX calculates this parameter by finding the strongest received signal power from any of the transmitters (base stations or access points). Then calculates the sum of the received signal powers from all the other transmitters which also have relevant signal levels. After the sum of the interference is found, the noise power is calculated. Several factors are taken into account in making this calculation such as directional receive antenna, cross-polarization rejection, transmitter power spectral density, receive filter and MIMO and adaptive 
antennas [98]. This study is used to identify and calculate potential co-

channel interference in areas where more than one AP/BS has coverage.

\section{Network design for a 10-year period}

Since the study is conducted for the deployment and operation of wireless access networks for a 10-year period, the design includes meeting customer and

speed demand growth over this period, which will lead to expanding and increasing the capacity of the access network (backhaul and access radio links). As the network size and capacity increases, the engineering cost model will also incorporate associated deployment and operating costs of such increase regarding to backhaul and access equipment, energy, and infrastructure, among others. Figure 14 to Figure 17 show the evolution of the wireless broadband access network using the Wi-Fi technology for the Yauli county study area from year 1 to year 10 . As the broadband customer and speed demand increases over the 10-year period, additional nodes and outdoor APs and backhaul radio links are deployed.

\section{Terrestrial and stratospheric access network design and simulation}

This study used the EDX software to simulate both terrestrial and stratospheric wireless access networks. This software already provides tutorials and tools to conduct terrestrial wireless network designs and simulations for popular commercial technologies such as Wi-Fi, WiMAX and LTE, among others. These types of wireless networks are currently the most common deployments carried out by wireless broadband service or mobile service operators. On the other hand, designing and simulating stratospheric wireless access networks are not 
commonly carried out by operators and EDX does not have tools per se to accomplish these tasks yet. However, EDX has some tools for satellite footprint analysis and simulation, and also allows setting tower heights of several kilometers above the ground. Using these tools allowed this study to conduct design and simulations of stratospheric access networks which, for example, placed micro-LTE base stations at an altitude of 20 kilometers (around 12.5 miles) above the ground. These stratospheric networks will be deployed using unmanned aerial vehicles (i.e. stratospheric balloons and aircrafts) as deployment and operating platforms. Figure 15 shows the coverage of a micro-LTE base station in the Yauli county study area. This simulation set the altitude of the base station at 20 kilometers above the ground, and it can be observed areas of coverage for the different speed data rates.

The results of the technical analysis are presented in detail in the next chapter. The results include detailed lists of access network design elements (i.e. number of nodes, towers, APs or BSs, backhaul radiolinks, CPEs, energy requirement, etc.) for each of the analyzed technologies for a 10-year period. These results are used as inputs in the engineering cost analysis as will be described in the next section. Other results, used for network technical performance analysis, include EDX simulation results such as area coverage at different broadband speeds (which depends on modulation and coding schemes for the different wireless technologies) and percentage of service availability in the coverage areas. 




Figure 14. Wireless broadband access network using outdoor Wi-Fi APs for the Yauli county study area from year 1 to year 3 


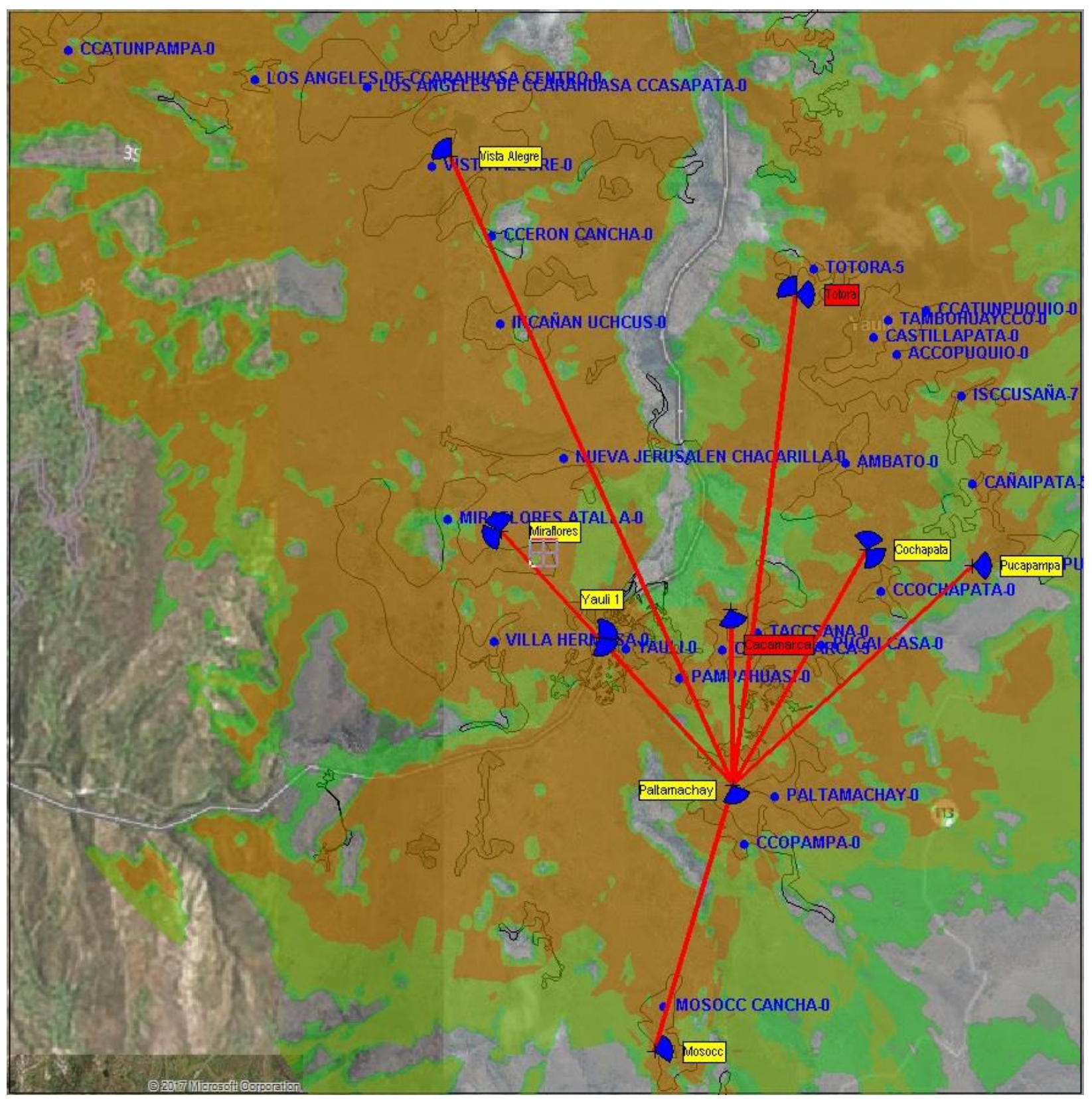

Figure 15. Wireless broadband access network using outdoor Wi-Fi APs for the Yauli county study area from year 4 to year 7 


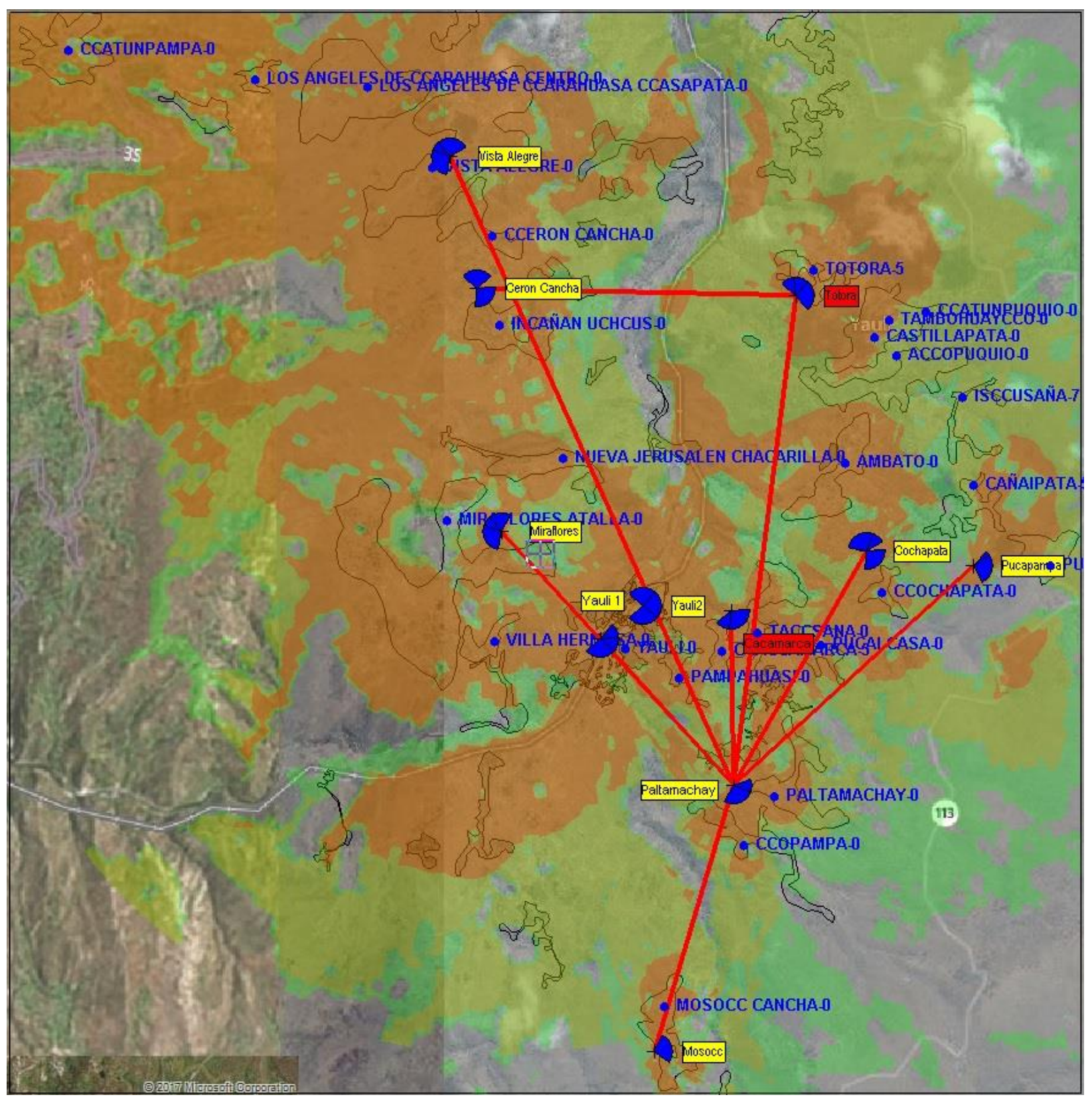

Figure 16. Wireless broadband access network using outdoor Wi-Fi APs for the Yauli county study area from year 8 to year 9 


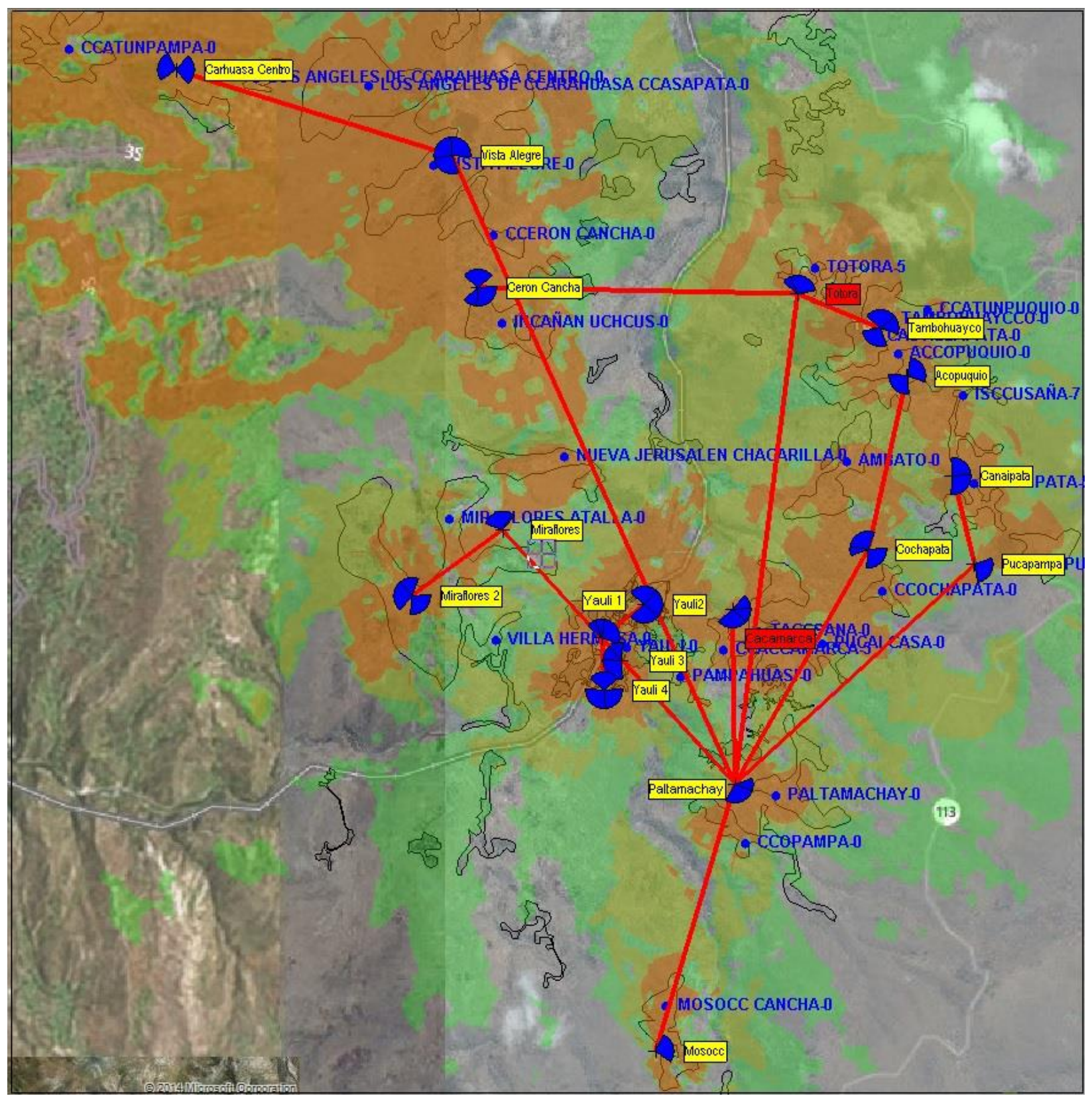

Figure 17. Wireless broadband access network using outdoor Wi-Fi APs for the Yauli county study area for year 10 


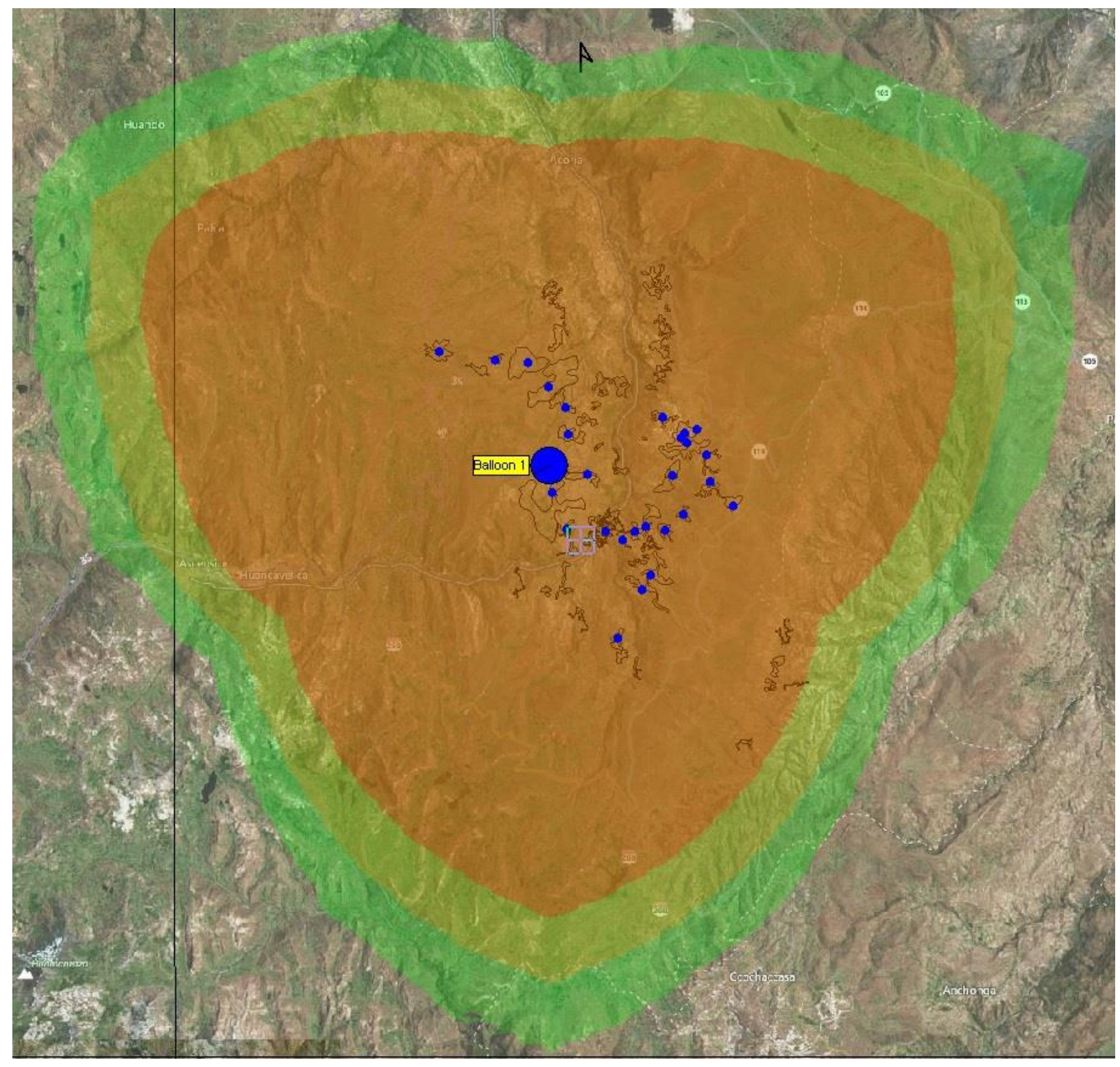

Figure 18. Footprint or coverage of a micro-LTE base station located at 20 kilometers above the ground in the Yauli county study area. 


\subsection{Engineering cost analysis: access network deployment and operation cost model in Analytica}

This section describes the engineering cost analysis which uses a bottom up cost model to estimate deployment and operating costs of the wireless access networks for a 10-year period. This cost model uses, first, the results of the technical analysis such as detailed list of network elements, second, cost data of these network elements (i.e. RF equipment, infrastructure, towers, energy system, etc.) and operating expenses (i.e. personnel, energy bills, interconnection, etc.), and third, other relevant cost variables (i.e. cost trends for wireless broadband technologies, interest rates, etc.). This study developed the bottom up cost model using the Analytica software. This section describes this cost model and detailed information of the cost data. The results of the engineering cost analysis includes: 1) total deployment and operating costs for a 10 -year period, 2) net present value of the total cost, 3) cost per user per month, and 4) cost per Mbps per month. The results of the engineering cost analyses are presented in the next chapter.

\subsubsection{Engineering cost model design in Analytica}

The engineering cost model was developed using a bottom up approach using Analytica. The bottom up approach allows estimating the infrastructure investment of an engineering type model. It assumes that a network starts from scratch, and network requirements and elements need to be defined and calculated respectively. Some advantages of this approach, preferred by regulators, are [99]: 
1) allowing setting Working Average Cost of Capital $($ WACC $)=$ Interest on Investment, and 2) it is useful if there is not much information about the existing network infrastructure (in the case of an already deployed network).

Disadvantages of this model are: 1) it is harder to compare against the actual costs structure of an operational network, 2) it is more complex to implement than the top down approach, and 3) results might not agree with other financial analysis. However, these disadvantages mostly apply in the case of using a bottom up model to estimate the cost structure of an already deployed and operational network which has undergone several upgrades of network elements and topology over years and run different services and networking technologies (i.e. cellphone and broadband service networks running over legacy networks). In this study, the cost model focuses on the cost structure of a green field wireless access network, which will be designed and deployed from scratch due to the lack of legacy infrastructure in unserved and underserved rural areas (focus of the FITEL bids and also this study). Then, this study develops and uses a bottom up cost model for the engineering cost analysis.

For the cost model, two main categories were defined: capital expenditures (CAPEX) and operating expenditures (OPEX). CAPEX are defined as costs used to purchase or upgrade physical assets such as equipment, industrial buildings or property, and included the following categories and elements:

- Tower infrastructure: Steel infrastructure used to mount the AP/BSs and antennas, and includes pre-installation ground studies per site, foundations 
and tower installation, and, lightning protection and grounding system. In the case of UAVs, infrastructure includes the aircraft or super-pressure balloon platform.

- Civil engineering: Node facilities to host equipment, cabinets, energy system, and includes pre-construction studies per site, land and land registration, node construction and construction license, equipment cabinet, access security system, and installation of equipment.

- Energy system: The energy system in each node that connects to the public power grid and also has a backup system in case the power grid fails. It includes a backup diesel generator (only for district node), control board, rectifier, peak protection system, backup batteries and grounding system.

- Network equipment: RF equipment (active and passive) for the backhaul radio links (point to point equipment), access radio links (AP/BSs), external outdoor antennas and coaxial cables, controllers, switches, and installation of this equipment. It also includes spare radios in case of failure or maintenance.

- Customer premise Equipment (CPE): RF equipment for government and residential clients. It includes installation and spare CPE in case of failure or maintenance.

OPEX are defined as costs incurred through the normal day by day operation such as rent, equipment, inventory costs, payroll, insurance and research and development costs. The OPEX included the following categories and elements: 
- General headquarters: headquarters rental, phone service, water, electricity, cleaning services, office supplies, transport, gasoline, lodging and storage, and repairs, among others.

- Nodes electricity bill: Nodes electricity bills for energy consumption of the $\mathrm{RF}$ equipment.

- Personnel payroll: Includes network manager, engineers and technicians.

- Local operation and authorizations fees: Broadband service operator license fees and business operation authorizations.

- Fiber optic backhaul interconnection fees: Interconnection fees for access to the optic fiber backbone.

This cost model based on CAPEX and OPEX reflects the deployment and operation costs of the access networks for the analyzed technologies, and as the network expands or increases its capacity (adding more nodes, backhauls or base stations) to handle demand growth over the 10 year period, the model will translate the network expansion design into costs. As mentioned before, the cost model uses the technical design results (detailed list of network elements per year) as inputs along with equipment cost data, among others.

Analytica was used to develop and run this cost model. Analytica is a visual software environment for building quantitative decision models, which among its main features allows building complex models and organizing them as a hierarchy of comprehensive modules [100]. Figure 19 shows the input modules (CPE, Network cost, Tower cost-infrastructure, Civil engineering cost and Energy system 
cost) for the CAPEX in the cost model, and the sub-modules and links part of the Network cost module. It can be seen that the CAPEX module connect to the WBAN cost module, which calculates the total Wireless Broadband Access Network cost based on inputs from the CAPEX and OPEX modules.

The cost model in Analytica was set to run for all technologies in parallel and for a 10-year period. Outputs for all modules will show cost results for all technologies from year 1 to year 10. The following subsection presents the cost data used for the model, including sources and data processing. 


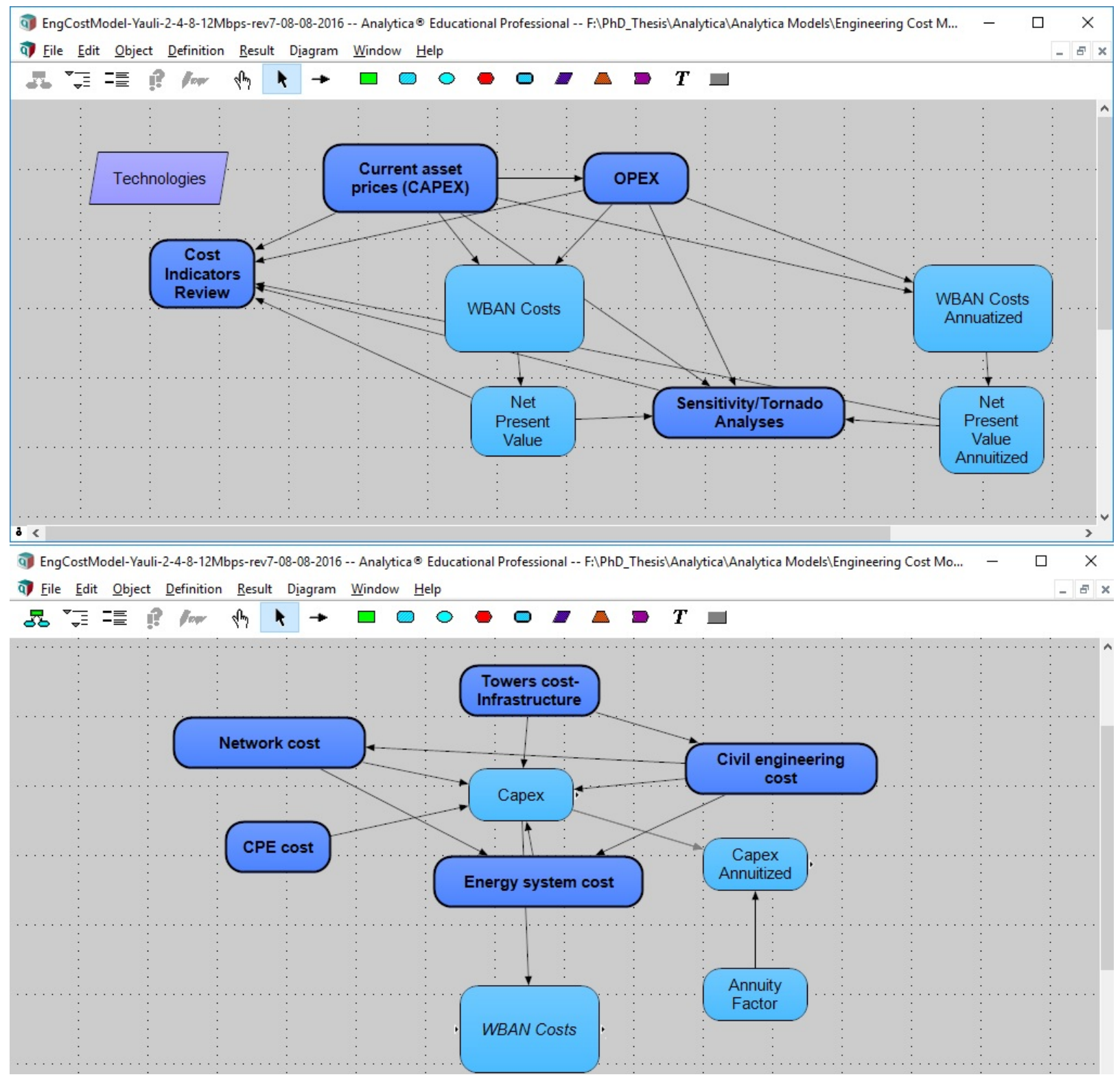

Figure 19. Modules in Analytica of the engineering cost model for the WBLANs. (Top) Total WBLAN cost modules. (Bottom) CAPEX modules.

\subsubsection{Cost data collection and processing}

\section{Wireless broadband equipment cost data}

For the collection of cost data of wireless broadband equipment, the first step was to identify main vendors for each technology. For this purpose, market reports, 
research papers, technology interest group websites, and broadband experts' blogs, among others, were reviewed. Next, state-of-the-art equipment and specific models were identified in vendor websites. Finally, vendor representatives (local representatives in Peru, regional representatives in Latin America or world-wide representatives) were contacted in order to request quotations and technical specifications of the selected equipment models.

In the first round of equipment quotation inquiries, most vendors wanted to learn more about this study and the inquiry. Then, phone calls and Skype conferences were set up with vendor representatives in order to explain the purpose of the inquiry. We provided truthful and accurate details of this academic study and the expected results which can be summarized as follows:

"Conducting a technical and engineering cost analysis to develop a broadband technology roadmap (in the form of a dissertation, research papers and reports) for introducing broadband services in rural areas in the Andes and Amazon regions of Peru, which will provide valuable information for regulators, broadband service providers, technology companies and entrepreneurs in Peru, and potentially in Latin America and other developing countries around the world. The research question was discussed with the universal service agency FITEL in Peru, and this academic research, which does not include actual deployments, was funded in part by a research grant from Google."

After holding the calls, most contacted vendors regarding WIMAX and TVWS equipment were interested in providing cost data and technical 
specifications to support this study and sent quotations and datasheets a few days later. In the case of LTE equipment, vendor representatives were not interested neither before nor after the calls and mentioned they dealt mostly with mobile operators (which hold LTE spectrum licenses) and needed to discuss actual potential purchases in order to send quotations. It was mentioned also that many factors are involved in setting the price of LTE base stations which might include equipment bundles (i.e. purchases including complementary equipment, accessories, maintenance, tech support and equipment upgrades) and purchase financing (i.e. 5 or 10 years financing), among others. In the case of Wi-Fi equipment, it was not necessary to set conference calls. Wi-Fi vendor representatives sent catalogs which included prices.

Several more rounds of quotations inquiries were conducted in order to gather additional LTE equipment cost data and also increase the cost data base for the other technologies. Three approaches were taken for these additional rounds: 1) contacting vendors in manufacturing locations (for example using Alibaba website to request LTE and Wi-Fi accessories equipment quotations from manufacturers in China), 2) searching whole-sale equipment vendors in specialized technology equipment websites in different countries (Huanetwork in China and Winncom in the United States), and 3) contacting vendor personnel through professional networking and requesting unofficial reference prices for specific equipment models. These additional approaches allowed collecting AP/BS and CPE cost data for the analyzed wireless broadband technology options from different vendors. 
Cost data was also collected, using all the aforementioned approaches, for wireless broadband backhaul equipment which included high throughput point to point equipment using microwaves, Wi-Fi, WiMAX or millimeter-wave technologies.

\section{Cost data processing for RF equipment}

Based on the cost data collection approaches mentioned above, a broad range of cost data for the different wireless broadband equipment was gathered; however, quotations and prices were based on different pickup/delivery conditions such as the following:

- Delivery/pick up in Lima, Peru: Price includes equipment is delivered/picked up in Lima.

- Carriage and Insurance Paid (CIP) at specific location/country (commonly at manufacturing country) Incoterms 2010: The quoted prices are on net basis only. The prices exclude, without limitation, any transportation or shipping charges, taxes, withholdings, duties or levies of any kind any other local duties, etc., which could be imposed on the equipment, S/W, and / or services in US/Peru.

- Ex works (EXW) at specific location/country (commonly at manufacturing plant): Under this price agreement, the seller is required to make goods ready for pickup at his or her own place of business or manufacturing plant. All other transportation costs and risks are assumed by the buyer. 
Then based on these delivery/pick up conditions, the cost data was processed to include the following adjustments when required:

- Freight/Shipping to Peru: Freight/shipping prices from seller/manufacturing location to Lima, Peru. It was selected maritime shipping due to its lower price in comparison to air shipping. Additionally, placing orders in advance (few weeks) to network deployment might not pose any deployment delay and RF equipment is among the last to be deployed after carrying out the construction of nodes, deployment of towers and installation of the energy system. This study estimated the maritime shipping price using shipping company websites and entering departure and arrival ports, and, price, weight and cost of the load.

- RF equipment import taxes and fees in Peru: It includes general sales tax, municipal (at port in Lima) promotion tax, $\mathrm{RF}$ equipment registration fee, RF equipment entrance fee, and customs agent fee.

A final adjustment to the cost data was also conducted to RF equipment and balloon components based on the purchase volume discount. In the case of equipment quotations, vendor prices were based on the number of units inquired, which was set according to the equipment required for the network deployment or expansion. For example, for the initial deployment, the access network would require tens of base stations or hundreds of $\mathrm{CPE}$. In the case of cost data based on a list price (from vendor websites or catalogs) per unit or small amounts, a volume discount was deducted from this list price, assuming that an actual purchase would 
come with this discount. This volume discount ranged from $10 \%$ to $30 \%$ of the price based on experts input.

\section{Towers, civil engineering and energy system cost data}

The cost data related to towers, civil engineering (node construction), and energy system was collected from local vendors or contractors in Peru. Due to the cost, weight and volume size of raw materials, and required labor for integration or construction, it is a common practice by telecom service operators and contractors to purchase the towers and civil engineering categories within the countries where networks will be deployed. Regarding the energy system category, Peru has a competitive energy equipment market with a wide range of vendors. Then, cost data for this category was also collected from local vendors in Peru. Sources for these categories include: Investment Fund in Telecommunications (FITEL), telecom network deployment contractors and engineering research groups working on wireless access networks in rural areas in Peru. FITEL shared vendor quotations and reference prices used in feasibility analysis for previous bids.

\section{Stratospheric super-pressure balloons cost data}

Data collection for the case of UAVs and more specifically for the stratospheric super-pressure balloons required a different approach since this technological solution is still in development stage with pilots running around the world. The Project Loon, run by Google X, has not released detailed costs of each balloon system or associated operating costs, it's only been released that the cost of each balloon system is of "tens of thousands" [54]. Then, based on publically 
available information from a patent filed by the Project Loon, and technical and cost information released to the press, this study estimated the cost of each balloon system by putting together the cost of individual components of each subsystem (i.e. envelope, altitude control communications, computational, navigation, and energy). The search for components cost was conducted on vendor websites. The SWAP+E (Space, Weight and Power + Environment) selection criteria was applied to selected the components for the balloon's payload. The SWAP+E criteria is used in the aerospace, avionics and satellite industry due to the different constraints posed by launching and operating a payload in a platform [101]. Then, based on the SWAP+E criteria, state-of-the-art components were selected and cost data of these components was gathered. Most cost data for the balloon's subsystems components were found on websites of vendors in the United States. Detailed information about the subsystems, components and cost data of the balloon systems is provided in the

\section{Appendix 2: High Altitude Platforms (HAPs) for Providing Broadband} Services. 


\section{CHAPTER IV}

\section{TECHNICAL AND ENGINEERING COST ANALYSIS RESULTS}

\subsection{WBLAN design and EDX simulation results - Yauli (Andes Region) and Samugari (Amazon Region) study areas}

\subsubsection{Current technologies: Wi-Fi, WiMAX, LTE and TVWS}

The technical analysis consisted of designing wireless broadband local access networks (WBLANs) using current wireless technology options (Wi-Fi, WiMAX, LTE and TVWS) and simulating these networks in EDX. Figure 20 shows the EDX coverage simulations for the WBLAN for the Yauli County using micro-LTE base stations for years 1 to 3. Appendix 4: EDX Simulation Results for Yauli and Samugari shows more detailed simulation results and also the evolution of this network during the 10-year period to handle growing customer and speed demands. As mentioned in the previous chapter, initial customer demand for fixed wireless broadband service in the Yauli County is $4.7 \%$ of the households with $2.7 \%$ annual growth, and in the Samugari County is $9.1 \%$ with $2.9 \%$ annual growth. In both areas, the initial average broadband speed demand per customer is $2 \mathrm{Mbps}$ (Y1-Y3), increasing to 4Mbps (Y4-Y7), 8Mbps (Y8-Y9) and 12Mbps (Y10). 


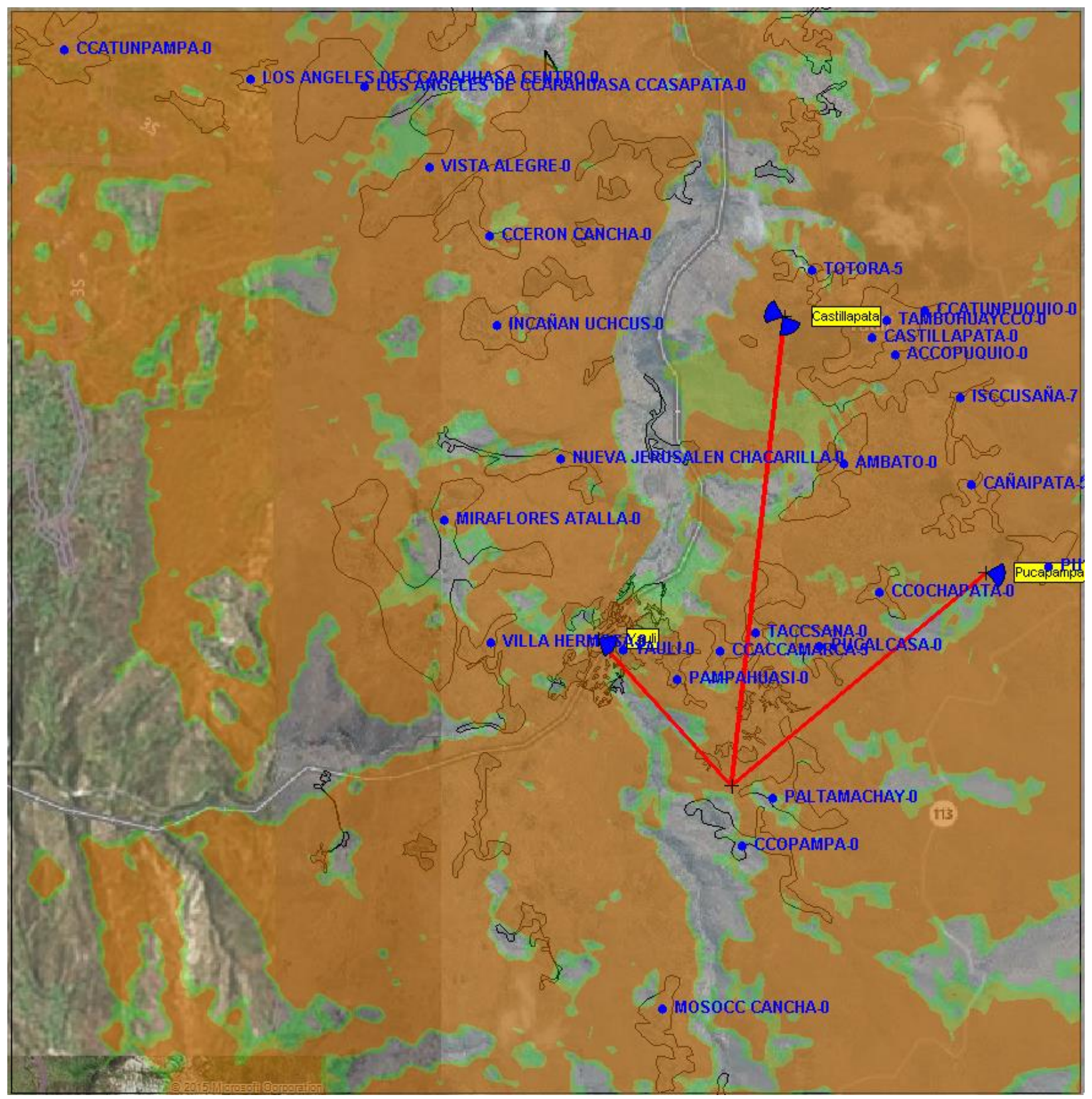

Figure 20. WBLAN using mini-LTE BSs for Yauli from year 1 to year 3.

The EDX simulations also show the coverage areas for different modulation rates. Figure 20 shows, for the LTE case, areas operating at 64QAM in red, at 16QAM in orange and at QPSK in green. It can be observed that higher modulation rates, and consequently higher download data rates, are achieved in areas closer to 
the base stations. The EDX query tools allow quantifying the populated areas (plotted town areas) operating at each data rate (assuming that customers are uniformly distributed in each town area). The estimated proportion of customers operating at different data rates was used to calculate the effective download channel capacity per base station (or access point) for the different technologies. This calculation also discounted the overhead used by each technology (or standard) and included only the actual throughput. The detailed calculation of the channel capacity is provided in Appendix 3: Calculation of the Effective Download Channel Capacity. Then the capacity per node (either in Mbps or number of customers) was calculated using the effective download channel capacity for each technology, average download broadband speed per customer, number of available channels and overbooking ratio (see formula in Section 3.2.5 Spectrum resources, reuse factor and overbooking). When nodes reach maximum capacity, the network design requires adding more nodes (including both base stations and backhaul equipment) and reducing the coverage of previous nodes to accommodate the new nodes and to avoid co-channel interference.

Table 2 shows the effective download channel capacity per AP/BS and download capacity per node for Wi-Fi, WiMAX, LTE and TVWS at different broadband speeds per customer in the Yauli area (Andes). In the technical analysis in both study areas, Wi-Fi (2.4GHz), WiMAX (3.5GHz), LTE (1.7/2.1GHz), and TVWS (470-698MHz) uses $20 \mathrm{MHz}, 10 \mathrm{MHz}, 20 \mathrm{MHz}$, and $6 \mathrm{MHz}$ channels, respectively. In Yauli, Wi-Fi achieves an effective download channel capacity from 
45.2Mbps to 50.9Mbps, WiMAX, from $35.6 \mathrm{Mbps}$ to $36.4 \mathrm{Mbps}$, LTE from $106.3 \mathrm{Mbps}$ to $107.8 \mathrm{Mbps}$, and TVWS from $9.9 \mathrm{Mbps}$ to $10.8 \mathrm{Mbps}$. LTE has the highest effective download channel capacity; this is due to LTE's high data rates and more throughput efficiency (less overhead) than the other technologies. Regarding the download capacity per node, this table shows that LTE also has the highest capacity per node due to the reuse of the $20 \mathrm{MHz}$ spectrum in three sectors within the same node, achieving total effective download capacity per node from 271.9Mbps to 291.0Mbps. WiMAX has the second highest effective download capacity per node due to its high effective download channel capacity and also using five channels (more than WiFi), achieving total effective download capacity per node from $178.1 \mathrm{Mbps}$ to $181.8 \mathrm{Mbps}$. TVWS has the third highest effective download capacity per node, from $149 \mathrm{Mbps}$ to $161.9 \mathrm{Mbps}$, due to having the most available channels, 15, overcoming having the lowest effective download channel capacity. TVWS is closely followed by Wi-Fi, from $135.6 \mathrm{Mbps}$ to $152.8 \mathrm{Mbps}$, which has higher effective download channel capacity than WiMAX and TVWS but only has three channels available. 
Table 2. Effective download channel and node capacity for Wi-Fi, WiMAX, LTE and TVWS in Yauli.

\begin{tabular}{|c|c|c|c|c|c|c|c|}
\hline & \multicolumn{3}{|c|}{ Channel Capacity } & & \multicolumn{3}{|c|}{ Node Capacity } \\
\hline $\begin{array}{c}\text { Avg. BB } \\
\text { Speed } \\
\text { Per } \\
\text { CPE }\end{array}$ & $\begin{array}{c}\text { DL } \\
\text { Chan } \\
\text { BW } \\
(\mathrm{MHz})\end{array}$ & $\begin{array}{c}\text { Eff. DL } \\
\text { Chan } \\
\text { Capacity } \\
\text { (Mbps) }\end{array}$ & $\begin{array}{c}\text { Max. \# } \\
\text { Users Per } \\
\text { Chan } \\
(10: 1)\end{array}$ & $\begin{array}{c}\text { \# of DL } \\
\text { Chan } \\
\text { (Available } \\
\text { Spectrum) }\end{array}$ & $\begin{array}{c}\text { DL BW } \\
\text { Per Node } \\
(\mathrm{MHz})\end{array}$ & $\begin{array}{c}\text { Eff. DL } \\
\text { Capacity } \\
\text { (Mbps) }\end{array}$ & $\begin{array}{c}\text { Max. \# } \\
\text { Users Per } \\
\text { Node } \\
(10: 1)\end{array}$ \\
\hline \multicolumn{8}{|c|}{ Wi-Fi } \\
\hline $2 \mathrm{Mbps}$ & 20 & 50.9 & 255 & 3 & 60 & 152.8 & 765 \\
\hline $4 \mathrm{Mbps}$ & 20 & 50.9 & 127 & 3 & 60 & 152.8 & 381 \\
\hline 8Mbps & 20 & 47.5 & 59 & 3 & 60 & 142.6 & 177 \\
\hline 12Mbps & 20 & 45.2 & 38 & 3 & 60 & 135.6 & 114 \\
\hline \multicolumn{8}{|c|}{ WiMAX } \\
\hline $2 \mathrm{Mbps}$ & 10 & 35.6 & 178 & 5 & 50 & 178.1 & 890 \\
\hline $4 \mathrm{Mbps}$ & 10 & 36.4 & 91 & 5 & 50 & 181.8 & 455 \\
\hline $8 \mathrm{Mbps}$ & 10 & 36.2 & 45 & 5 & 50 & 181.2 & 225 \\
\hline $12 \mathrm{Mbps}$ & 10 & 35.6 & 30 & 5 & 50 & 178.1 & 150 \\
\hline \multicolumn{8}{|c|}{ LTE (reusing $20 \mathrm{MHz} \times 3 \times 90 \%$ per sector) } \\
\hline $2 \mathrm{Mbps}$ & 20 & 106.3 & 532 & 1 & 20 & 287.0 & 1436 \\
\hline $4 \mathrm{Mbps}$ & 20 & 100.7 & 252 & 1 & 20 & 271.9 & 680 \\
\hline $8 \mathrm{Mbps}$ & 20 & 107.4 & 134 & 1 & 20 & 290.0 & 362 \\
\hline $12 \mathrm{Mbps}$ & 20 & 107.8 & 90 & 1 & 20 & 291.0 & 243 \\
\hline \multicolumn{8}{|c|}{ TVWS } \\
\hline $2 \mathrm{Mbps}$ & 6 & 9.9 & 50 & 15 & 90 & 149.0 & 750 \\
\hline $4 \mathrm{Mbps}$ & 6 & 10.1 & 25 & 15 & 90 & 152.1 & 375 \\
\hline $8 \mathrm{Mbps}$ & 6 & 10.4 & 13 & 15 & 90 & 155.6 & 195 \\
\hline $12 \mathrm{Mbps}$ & 6 & 10.8 & 9 & 15 & 90 & 161.9 & 135 \\
\hline
\end{tabular}

Table 3 shows the effective download channel capacity and download capacity per node for Samugari. Wi-Fi achieves an effective download channel capacity from $54.2 \mathrm{Mbps}$ to $54.7 \mathrm{Mbps}$, WiMAX, from $36.5 \mathrm{Mbps}$ to $36.7 \mathrm{Mbps}$, LTE, 
from $101.4 \mathrm{Mbps}$ to $108.5 \mathrm{Mbps}$, and TVWS, from $10.6 \mathrm{Mbps}$ to $10.8 \mathrm{Mbps}$. The

effective download channel capacity for the four technologies is slightly higher than in the Yauli area due to, first, towns having a smaller area (houses are clustered around a main square and almost none area located in the open jungle), and second, having more nodes to place BS/AP closer to towns. This table shows that Wi-Fi has the highest effective download channel capacity, closely followed by LTE. Regarding the download capacity per node, this table shows that in this area LTE has also the highest capacity per node, 273.9Mbps to $293.1 \mathrm{Mbps}$, followed by WiMAX , $182.4 \mathrm{Mbps}$ to $183.6 \mathrm{Mbps}$. Then Wi-Fi, $162.5 \mathrm{Mbps}$ to $164.1 \mathrm{Mbps}$, which is closely followed by TVWS, $159.6 \mathrm{Mbps}$ to $161.9 \mathrm{Mbps}$, due to having the most available channels, 15, overcoming having the lowest channel capacity.

Another factor to include in the node capacity analysis is that as the average broadband customer speed increases during the 10-year period, each customer requires more bandwidth, and as a result, each node is able to serve smaller number of users along this 10-year period, shown in Figure 21 for the Yauli area. This figure shows the number of users and Mbps capacity per node for Wi-Fi, WiMAX, LTE and TVWS. LTE nodes can handle more users than the other technologies and also provide the most bandwidth (Mbps), followed by WiMAX and then TVWS and Wi-Fi. WiMAX, TVWS and Wi-Fi have more download spectrum available $(50 \mathrm{MHz}, 90 \mathrm{MHz}$ and $60 \mathrm{MHz}$ respectively) than LTE $(20 \mathrm{MHz})$; however LTE allows reusing the same spectrum using sectors. Current LTE deployments use three sectors, which assuming an efficiency of $90 \%$ per sector allows a $2.7 \mathrm{x}$ 
capacity increase for the $20 \mathrm{MHz}$ download spectrum. Based on spectrum efficiency metrics, also shown in Figure 21, LTE also serves most users and provides most Mbps per MHz than the other technologies.

Table 3. Effective download channel and node capacity for Wi-Fi, WiMAX, LTE and TVWS in Samugari

\begin{tabular}{|c|c|c|c|c|c|c|c|}
\hline \multirow[b]{2}{*}{$\begin{array}{c}\text { Wi-Fi } \\
\text { Avg. BB } \\
\text { Speed } \\
\text { Per } \\
\text { CPE }\end{array}$} & \multicolumn{3}{|c|}{ Channel Capacity } & \multirow[b]{2}{*}{$\begin{array}{c}\text { \# of DL } \\
\text { Chan } \\
\text { (Available } \\
\text { Spectrum) }\end{array}$} & \multicolumn{3}{|c|}{ Node Capacity } \\
\hline & $\begin{array}{c}\text { DL } \\
\text { Chan } \\
\text { BW } \\
(\mathrm{MHz})\end{array}$ & $\begin{array}{c}\text { Eff. DL } \\
\text { Chan } \\
\text { Capacity } \\
\text { (Mbps) }\end{array}$ & $\begin{array}{c}\text { Max. \# } \\
\text { Users Per } \\
\text { Chan } \\
(10: 1)\end{array}$ & & $\begin{array}{c}\text { DL BW } \\
\text { Per Node } \\
(\mathrm{MHz})\end{array}$ & $\begin{array}{c}\text { Eff. DL } \\
\text { Capacity } \\
\text { (Mbps) }\end{array}$ & $\begin{array}{c}\text { Max. \# } \\
\text { Users } \\
\text { Per } \\
\text { Node } \\
(10: 1)\end{array}$ \\
\hline $2 \mathrm{Mbps}$ & 20 & 54.7 & 274 & 3 & 60 & 164.1 & 822 \\
\hline $4 \mathrm{Mbps}$ & 20 & 54.7 & 137 & 3 & 60 & 164.1 & 411 \\
\hline $8 \mathrm{Mbps}$ & 20 & 54.4 & 68 & 3 & 60 & 163.3 & 204 \\
\hline $12 \mathrm{Mbps}$ & 20 & 54.2 & 45 & 3 & 60 & 162.5 & 135 \\
\hline \multicolumn{8}{|c|}{ WiMAX } \\
\hline $2 \mathrm{Mbps}$ & 10 & 36.7 & 184 & 5 & 50 & 183.6 & 920 \\
\hline $4 \mathrm{Mbps}$ & 10 & 36.7 & 92 & 5 & 50 & 183.6 & 460 \\
\hline $8 \mathrm{Mbps}$ & 10 & 36.6 & 46 & 5 & 50 & 183.0 & 230 \\
\hline $12 \mathrm{Mbps}$ & 10 & 36.5 & 30 & 5 & 50 & 182.4 & 150 \\
\hline \multicolumn{8}{|c|}{ LTE (reusing $20 \mathrm{MHz}$ x 3 x 90\% per sector) } \\
\hline $2 \mathrm{Mbps}$ & 20 & 101.4 & 508 & 1 & 20 & 273.9 & 1372 \\
\hline $4 \mathrm{Mbps}$ & 20 & 101.4 & 254 & 1 & 20 & 273.9 & 686 \\
\hline $8 \mathrm{Mbps}$ & 20 & 108.2 & 136 & 1 & 20 & 292.0 & 367 \\
\hline $12 \mathrm{Mbps}$ & 20 & 108.5 & 90 & 1 & 20 & 293.1 & 243 \\
\hline \multicolumn{8}{|c|}{ TVWS } \\
\hline $2 \mathrm{Mbps}$ & 6 & 10.6 & 53 & 15 & 90 & 159.6 & 795 \\
\hline $4 \mathrm{Mbps}$ & 6 & 10.6 & 27 & 15 & 90 & 159.6 & 405 \\
\hline $8 \mathrm{Mbps}$ & 6 & 10.8 & 13 & 15 & 90 & 161.9 & 195 \\
\hline 12Mbps & 6 & 10.8 & 9 & 15 & 90 & 161.9 & 135 \\
\hline
\end{tabular}


Figure 22 shows the number of users and Mbps capacity per node for Wi-Fi, WiMAX, LTE and TVWS in the Samugari area. In this area, LTE nodes also have more capacity (users and Mbps) than the other technologies, followed by WiMAX and then Wi-Fi and TVWS. Based on spectrum efficiency metrics, LTE also serves most users and provides most Mbps per $\mathrm{MHz}$ than the other three technologies.
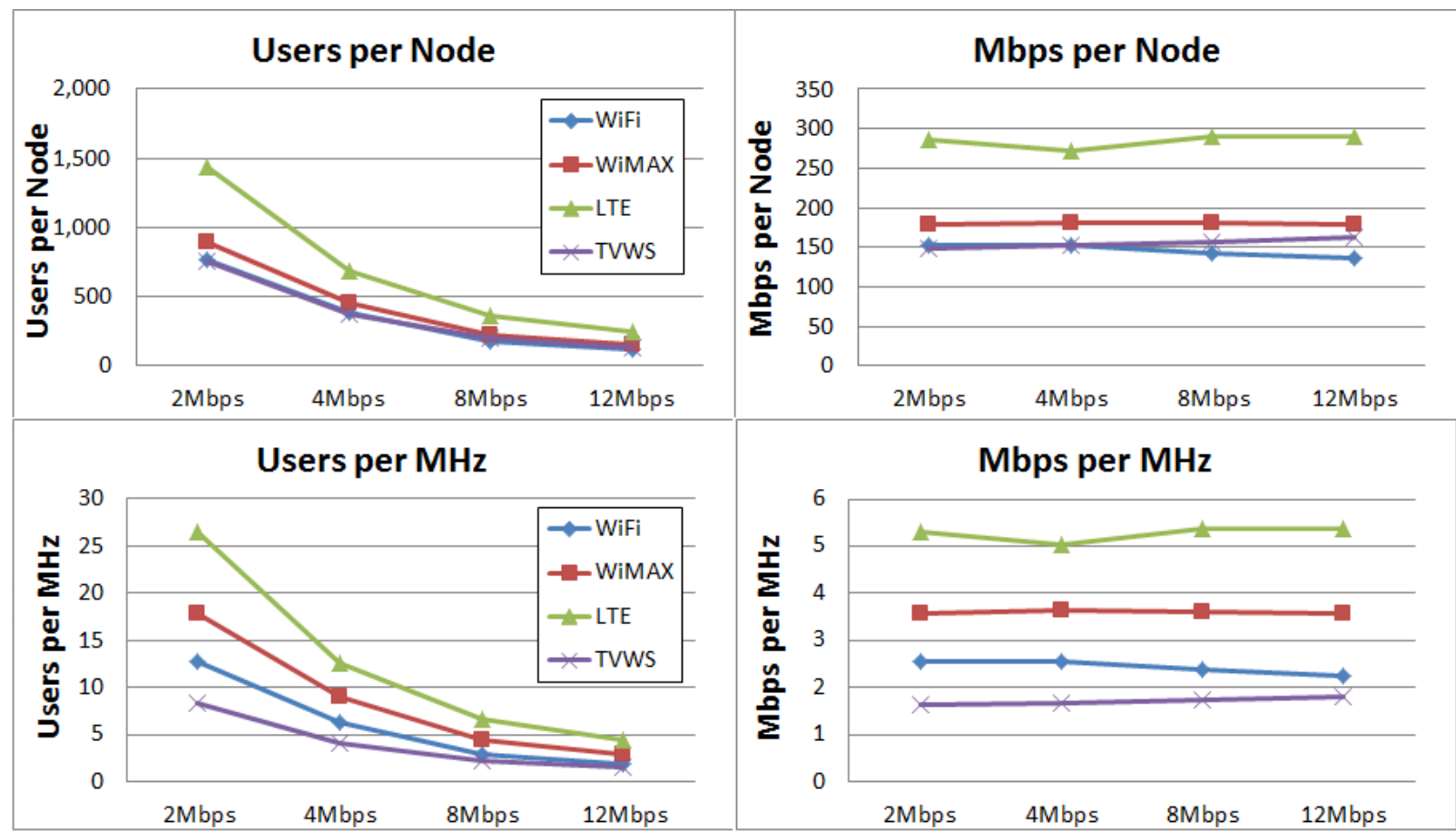

Figure 21. Users and Mbps capacity per node (Top) and users and Mbps per MHz (Bottom) for current wireless technology options in Yauli 

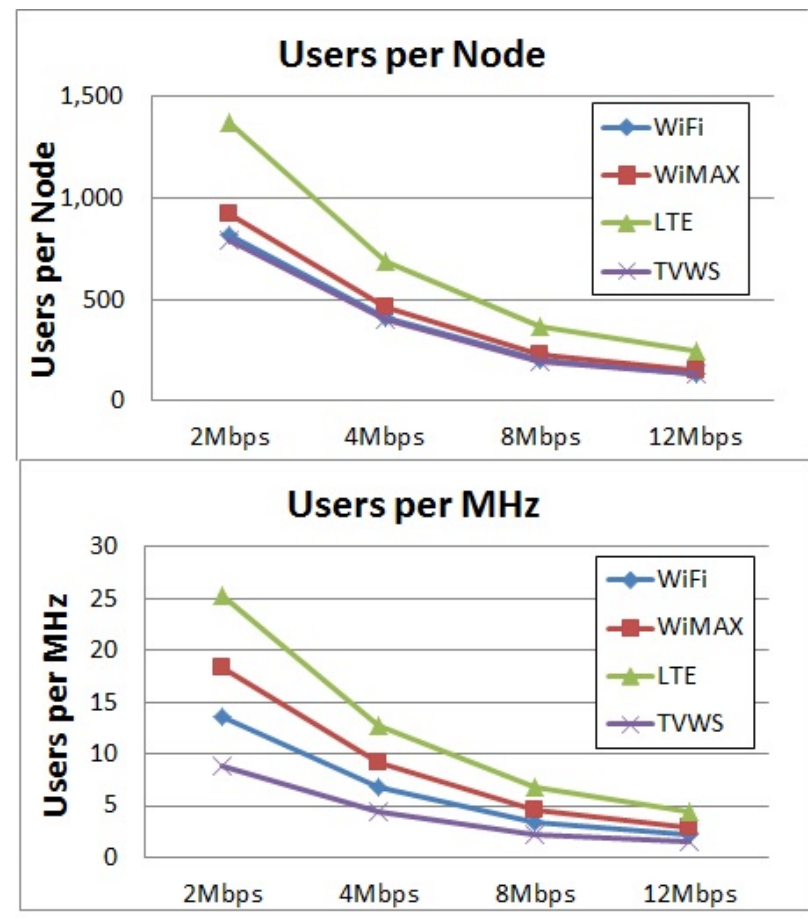

Figure 22. Users and Mbps capacity per node (Top) and users and Mbps per MHz (Bottom) for current wireless technology options in Samugari.

Table 4 shows the network elements (nodes, towers, AP/BSs, backhaul radios and CPEs) of the WBLAN design results for the Yauli area for Wi-Fi, WiMAX, LTE and TVWS. This table shows the results for years 1, 4, 8 and 10, which are the years when networks require capacity upgrades due to incremental steps on the average broadband speed demand (2Mbps, 4Mbps, $8 \mathrm{Mbps}$ and $12 \mathrm{Mbps})$. The towers used in this area for the point-to-point backhaul equipment and the $\mathrm{AP} / \mathrm{BSs}$ in each node required heights ranging from 21 to 30 meters (69 to 98 feet) and were located in elevated places to enable LOS over mountainous terrain for backhaul radio links.

In year 1, TVWS requires the least number of nodes, 2 , due to the TVWS operation band $(470-698 \mathrm{MHz})$, which allows propagation through or around 
obstacles (moderate vegetation and mountains). Both WiMAX and LTE require more nodes than TVWS, 4 , due to their operation at higher frequencies $(3.5 \mathrm{GHz}$ and 1.7/2.1GHz, respectively), and consequently smaller coverage (distance) per node than TVWS. However, these technologies can operate in near-line-of-sight (nLOS) or non-line-of-sight (NLOS) conditions which allow coverage of a few kilometers in this mountainous region. Wi-Fi requires the most number of nodes, 9 , due to the limited channel access mechanism to serve large number of CPEs at distances of more than a kilometer (which requires to activate additional collision avoidance features which increases the overhead and leads to a decrease in the effective download channel capacity), and the requirement of LOS or near-LOS between APs and CPEs. Regarding the number of AP/BSs, WiMAX and LTE require the least number, 4 , due to their high effective download channel capacity in comparison to TVWS, which require 5. Wi-Fi also has a high channel capacity, however, it requires more density of APs, 9 , and more nodes to provide the same coverage than the other technologies.

In year 4, LTE requires 4 nodes and WiMAX and TVWS require 5 nodes, showing a network expansion to satisfy the growing customer and speed demand. Wi-Fi requires the same number of nodes than in year 1, 9 nodes. TVWS requires the highest number of base stations, 23, due to its smaller effective download channel capacity in comparison to the other technologies resulting in a lower client load per base station. LTE and WiMAX require 4 and 9 base stations, respectively, 
due to having higher effective channel capacity. Wi-Fi can still serve the demand using 12 APs.

In year 8 , LTE requires the least number of nodes, 6 . TVWS and WiMAX require 8 nodes, while Wi-Fi requires 11 nodes. LTE requires the least number of base stations, 12 , due to the higher effective channel capacity per BS which results in a greater client load per base station. LTE is followed by Wi-Fi and WiMAX with 26 and 28 base stations, respectively. TVWS again requires the largest number of base stations, 85 .

In year 10, LTE requires the least number of nodes, 7 . TVWS and WiMAX require 12 nodes, followed by Wi-Fi with 18 nodes. LTE again requires the least number of base stations, 20, followed by Wi-Fi and WiMAX with 46 and 50 base stations, respectively. TVWS requires again the most number of base stations, 158 .

These design results show that LTE requires the least number of nodes and base stations due to having the highest effective download channel capacity and the highest capacity per node.

Table 5 shows the network elements (nodes, towers, AP/BSs, backhaul radios and CPEs) of the WBLAN design results for the Samugari area for Wi-Fi, WiMAX, LTE and TVWS. In this study area, the sparse location of the towns (separated from $2 \mathrm{~km}$ to $8 \mathrm{~km}$ ) and dense vegetation between towns prevents these wireless technologies from serving several towns with one node; resulting in an increased number of required nodes (around double) in comparison to the Andes scenario. Additionally, due to this rainforest landscape, the WBLANs required use 
of taller towers, from 30 to 60 meters (98 to 197 feet) in order to propagate above the tree canopy (15 to 20 meters height) for both the backhaul radio links and base stations. The rainforest poses as a major attenuation source.

Table 4. WBLAN design results for the Yauli county for the 10-year period for Wi-Fi, WiMAX, LTE and TVWS.

\begin{tabular}{|c|c|c|c|c|}
\hline & Wi-Fi & WiMAX & TVWS & LTE \\
\hline & \multicolumn{4}{|c|}{ Y1 (2Mbps) } \\
\hline Nodes & 9 & 4 & 2 & 4 \\
\hline Towers & 9 & 4 & 2 & 4 \\
\hline Base Stations/Access Points & 12 & 4 & 5 & 4 \\
\hline Backhaul Radio Links & 8 & 3 & 1 & 3 \\
\hline \multirow[t]{2}{*}{ Customer Premise Equipment } & 205 & 205 & 205 & 205 \\
\hline & \multicolumn{4}{|c|}{ Y4 (4Mbps) } \\
\hline Nodes & 9 & 5 & 5 & 4 \\
\hline Towers & 9 & 5 & 5 & 4 \\
\hline Base Stations/Access Points & 12 & 9 & 23 & 4 \\
\hline Backhaul Radio Links & 8 & 4 & 4 & 3 \\
\hline \multirow[t]{2}{*}{ Customer Premise Equipment } & 532 & 532 & 532 & 532 \\
\hline & \multicolumn{4}{|c|}{ Y8 (8Mbps) } \\
\hline Nodes & 11 & 8 & 8 & 6 \\
\hline Towers & 11 & 8 & 8 & 6 \\
\hline Base Stations/Access Points & 26 & 28 & 85 & 12 \\
\hline Backhaul Radio Links & 12 & 10 & 9 & 8 \\
\hline \multirow[t]{2}{*}{ Customer Premise Equipment } & 1064 & 1064 & 1064 & 1064 \\
\hline & \multicolumn{4}{|c|}{ Y10 (12Mbps) } \\
\hline Nodes & 18 & 12 & 12 & 7 \\
\hline Towers & 18 & 12 & 12 & 7 \\
\hline Base Stations/Access Points & 46 & 50 & 158 & 20 \\
\hline Backhaul Radio Links & 23 & 19 & 18 & 14 \\
\hline Customer Premise Equipment & 1368 & 1368 & 1368 & 1368 \\
\hline
\end{tabular}


In year 1 , TVWS requires the least number of nodes, 9 , due to the TVWS operation band (470-698MHz), which allows propagation through or around obstacles. However, dense vegetation in this geographical area prevents this technology to fully benefit from its improved propagation characteristics and cover several sparse towns with significantly lower number of nodes than the other technologies. Both WiMAX and LTE require 10 nodes in this year; only one more node than TVWS. Wi-Fi requires the most number of nodes, 18, due to the limited channel access mechanism to serve large number of CPEs at distances of more than a kilometer and the requirement of LOS or near-LOS between APs and CPEs. LTE requires the least number of BSs, 10, and Wi-Fi requires the most, 18.

In year 4 , the number of nodes remains the same for all technologies. This is due to the customer and speed demand not reaching the maximum capacity (users and Mbps) of the nodes. There is a slightly increase in the number of base stations for WiMAX, one more BS, and a major increase for TVWS, 19 more BSs. TVWS has the least effective download channel capacity so it has the lowest client load per BS, leading to significant increase in the BS/APs as customer and speed demand increase. LTE and Wi-Fi remain with the same number of BSs, 10 and 18, respectively.

In year 8, LTE requires the least amount of nodes, 10. TVWS and WiMAX require 12 nodes, while Wi-Fi requires 18 nodes. TVWS again requires the largest number of base stations, 111, due to its low client load. LTE requires the least 
number of base stations, 17, due to the higher average channel capacity per BS which results in a greater client load.

In year 10, LTE requires the least number of nodes, 12, followed by TVWS and WiMAX with 17 and 18 nodes, respectively, and then Wi-Fi with 22 nodes. LTE again requires the least number of base stations, 26, followed by Wi-Fi and WiMAX with 46 and 62 base stations, respectively. TVWS requires again the most number of base stations, 170 .

In Samugari, LTE also requires the least number of nodes and base stations due to having the highest effective download channel capacity and the highest capacity per node.

In both study areas, Yauli and Samugari, the technology used for backhaul links in the WBLAN design for all technologies was point-to-point WiMAX using the unlicensed $5.8 \mathrm{GHz}$ band. This technology was selected among a wide range of point-to-point equipment options including microwaves, long distance Wi-Fi, millimeter-wave, $900 \mathrm{MHz}$, and WiMAX. 
Table 5. WBLAN design results for the Samugari county for the 10-year period for Wi-Fi, WiMAX, LTE and TVWS.

\begin{tabular}{|c|c|c|c|c|}
\hline & Wi-Fi & WiMAX & TVWS & LTE \\
\hline & \multicolumn{4}{|c|}{ Y1 (2Mbps) } \\
\hline Nodes & 18 & 10 & 9 & 10 \\
\hline Towers & 18 & 10 & 9 & 10 \\
\hline Base Stations/Access Points & 18 & 14 & 13 & 10 \\
\hline Backhaul Radio Links & 17 & 9 & 8 & 9 \\
\hline \multirow[t]{2}{*}{ Customer Premise Equipment } & 413 & 413 & 413 & 413 \\
\hline & \multicolumn{4}{|c|}{ Y4 (4Mbps) } \\
\hline Nodes & 18 & 10 & 9 & 10 \\
\hline Towers & 18 & 10 & 9 & 10 \\
\hline Base Stations/Access Points & 18 & 15 & 32 & 10 \\
\hline Backhaul Radio Links & 18 & 10 & 9 & 10 \\
\hline \multirow[t]{2}{*}{ Customer Premise Equipment } & 803 & 803 & 803 & 803 \\
\hline & \multicolumn{4}{|c|}{ Y8 (8Mbps) } \\
\hline Nodes & 18 & 12 & 12 & 10 \\
\hline Towers & 18 & 12 & 12 & 10 \\
\hline Base Stations/Access Points & 27 & 34 & 111 & 17 \\
\hline Backhaul Radio Links & 25 & 19 & 19 & 17 \\
\hline \multirow[t]{2}{*}{ Customer Premise Equipment } & 1368 & 1368 & 1368 & 1368 \\
\hline & \multicolumn{4}{|c|}{ Y10 (12Mbps) } \\
\hline Nodes & 22 & 18 & 17 & 12 \\
\hline Towers & 22 & 18 & 17 & 12 \\
\hline Base Stations/Access Points & 46 & 62 & 170 & 26 \\
\hline Backhaul Radio Links & 38 & 34 & 33 & 29 \\
\hline Customer Premise Equipment & 1675 & 1675 & 1675 & 1675 \\
\hline
\end{tabular}




\subsubsection{Stratospheric Access Networks \\ Super pressure balloons (Project Loon)}

This technical scenario consists of providing fixed wireless broadband service in the Yauli and Samugari study areas deploying stratospheric WBLANs in the lower stratosphere using super-pressure balloons. Google X's Project Loon is currently leading and testing this stratospheric WBLAN approach. In this analysis, LTE is the technology used for the base station to connect to the CPE on the ground. A micro-LTE base station was selected to be carried in the payload of the balloon. For the backhaul, WiMAX point-to-point equipment operating in the unlicensed $5.8 \mathrm{GHz}$ was selected. This backhaul links the micro-LTE base station in the balloon to the district node in the ground and carries the bidirectional traffic. The district node has a fiber optic backbone point of presence (POP) which allows connecting the terrestrial and stratospheric WBLANs to the Internet.

Figure 23 shows the coverage of the micro-LTE BS carried in a stratospheric balloon $20 \mathrm{~km}$ above the ground in the Yauli area. This simulation shows coverage of three micro-LTE base stations (assuming a configuration similar to LTE terrestrial deployments in order to reuse spectrum using three sectors to achieve around $2.4 \mathrm{x}$ capacity increase) providing a footprint of around $40 \mathrm{~km}$ diameter; matching potential coverage of Project Loon's balloons mentioned in [102] [103]. The 27 towns in the Yauli area are located approximately within a $15 \mathrm{~km}$ diameter area. Due to the relatively small study area in comparison to the large balloon coverage area (around $40 \mathrm{~km}$ diameter), it would be technically challenging to use 
more than one balloon at a time (similar to adding one more node in the case of terrestrial networks and reducing the coverage of each node to reuse the spectrum). The CPEs' directive antennas have fixed alignment and are set to vertically point up to the sky, which due to a 20 degrees aperture, allows linking to the balloon before it is flying right over the CPEs. Then, since each balloon is already reusing the spectrum just as terrestrial networks do using three sectors, it would be challenging to have more than one balloon (each balloon bringing three additional sectors) flying over this study area using the same spectrum and focusing their base station's antenna beams only to a subset of specific towns and CPEs (even if beams are narrower than $40 \mathrm{~km}$ using more directive antennas). As a result, in the Yauli area, the technical analysis assumes only one balloon (carrying up to three base stations reusing the same spectrum) at a time flying over the study area and pointing a total of $40 \mathrm{~km}$ coverage towards this area. For the case of a constellation of balloons flying over the study area, the larger balloon coverage (in comparison to the study area) allows providing 1 hour of coverage (flying at slow speed) over the study area before the next balloon should arrive to continue providing broadband service coverage. 


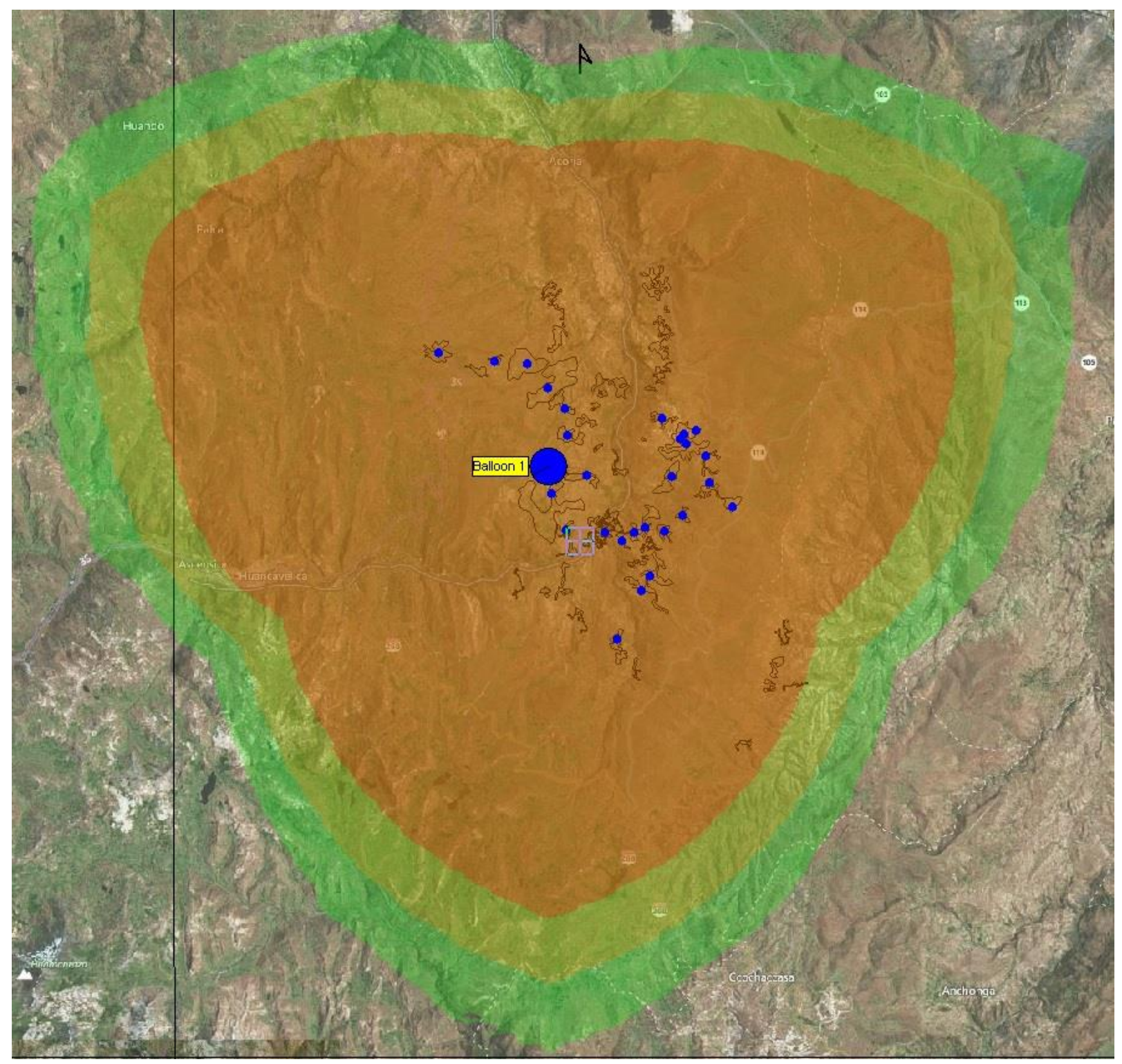

Figure 23. Coverage of a micro-LTE BS carried in a stratospheric balloon above the Yauli area. Towns are shown in blue. High, mid, and low data rate areas are shown in orange, yellow and green.

Figure 24 shows the coverage of the micro-LTE BS carried in a stratospheric balloon $20 \mathrm{~km}$ above the ground in the Samugari area. The 29 towns in this study area are located approximately within a $30 \mathrm{~km}$ diameter area. In this area, it might be feasible to use two balloons to provide broadband services; each balloon carrying up to three micro-base stations reusing the same spectrum. As shown in this 
figure, one balloon can serve towns in the northern area and the second balloon can serve towns in the southern area. Then, in this study area, the technical analysis assumes two balloons at a time flying over and pointing the coverage beams to the northern and southern towns.

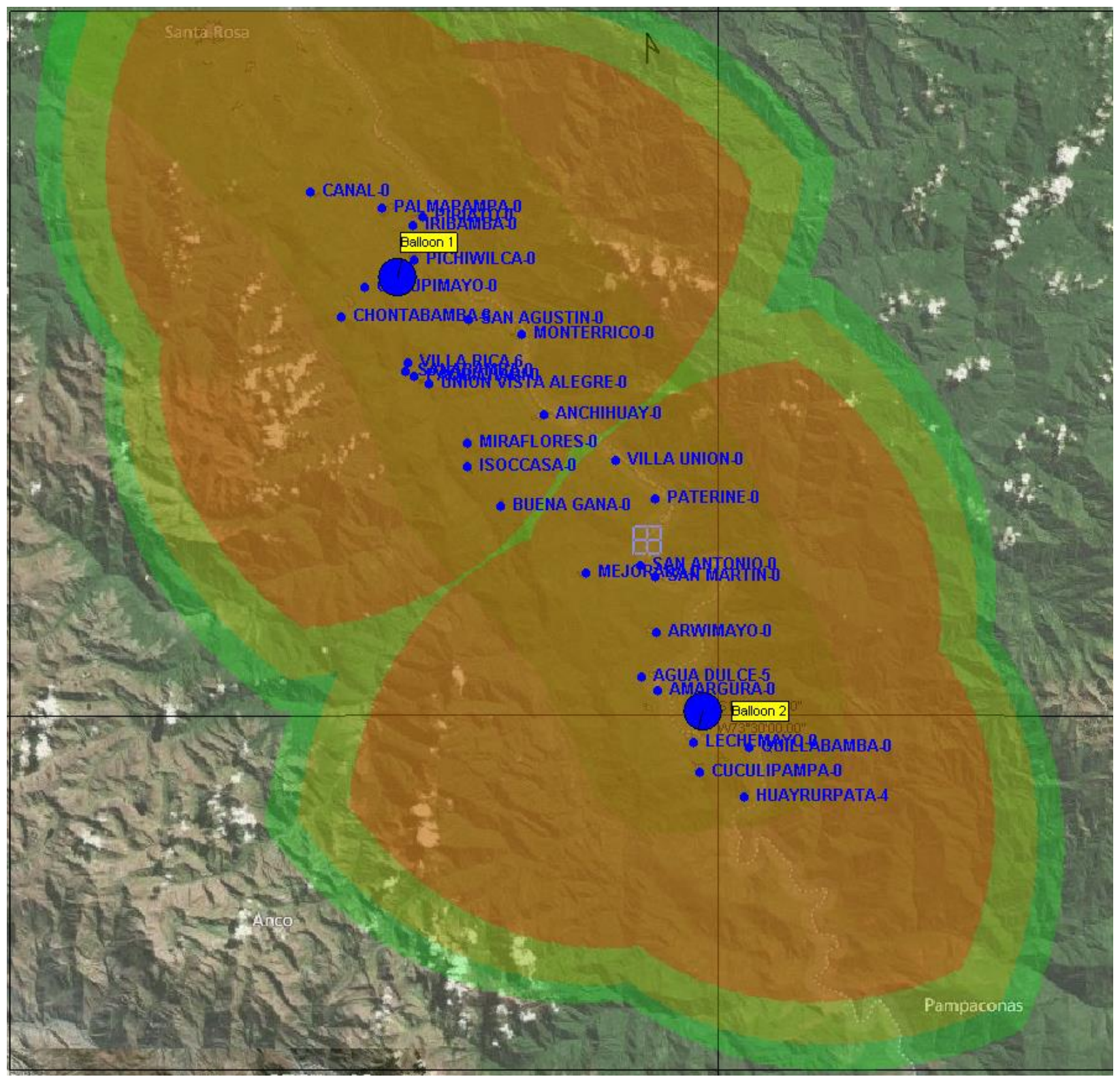

Figure 24. Coverage of a micro-LTE BS carried in a stratospheric balloon above the Samugari area. Towns are shown in blue. High, mid, and low data rate areas are shown in orange, yellow and green. 
For providing broadband service to both study areas, two balloon flight patterns are analyzed: 1) balloons flying permanently over the service area (one balloon in Yauli and two balloons in Samugari), and 2) a constellation of balloons flying over a path and passing over the service areas. In both flight pattern cases, continuous broadband service is provided to customers; matching the service provided using terrestrial WBLANs. Based on Project Loon's initial announcements and some recent flight tests in South America, the flight paths can be global, regional or local. The chosen path will have an impact on the number of balloons required to provide continuous service in the study areas, and as a result, it will also have an impact in the deployment and operating costs over the 10 -year period.

Under current Peruvian spectrum regulations, providing broadband service using LTE in stratospheric balloons allows using a maximum of $20 \mathrm{MHz}$ download spectrum per balloon (or per node). This technical analysis assumes a $20 \mathrm{MHz}$ download channel to maximize effective download channel capacity and minimize the number of base stations in the balloon payload. Additionally, the analysis assumes a similar reuse of the LTE spectrum just as terrestrial LTE network deployments. In the case of terrestrial LTE networks, each node reuses the spectrum using three sectors in order to achieve a theoretical capacity increase of $3 \mathrm{x}$. However, LTE deployment experts point that the capacity increase is around $2.7 \mathrm{x}$ accounting for a $90 \%$ capacity efficiency in each sector due to additional control access mechanism processing (10\% efficiency loss) in areas of coverage 
overlapping between two sectors. In the case of balloons, due to the continuous movement of the micro-LTE base stations, it is assumed that the capacity increase for using up to three micro-LTE base stations will be lower than in the terrestrial case; that is around $2.4 \mathrm{x}$ instead of $2.7 \mathrm{x}$ accounting for a $80 \%$ capacity efficiency per sector (20\% efficiency loss for coverage overlapping areas). In this scenario of reusing one LTE $20 \mathrm{MHz}$ download channel in three sectors to achieve a $2.4 \mathrm{x}$ capacity increase, balloons do not provide enough capacity to handle the customer and speed demands in either study area for the entire 10 -year period. Figure 25 shows the fixed broadband demand and the balloon's LTE capacity in the Yauli area. Then under current 1.7/2.1GHz LTE band regulations, in this area, one balloon can serve the customer and speed demands only for the first five years. 


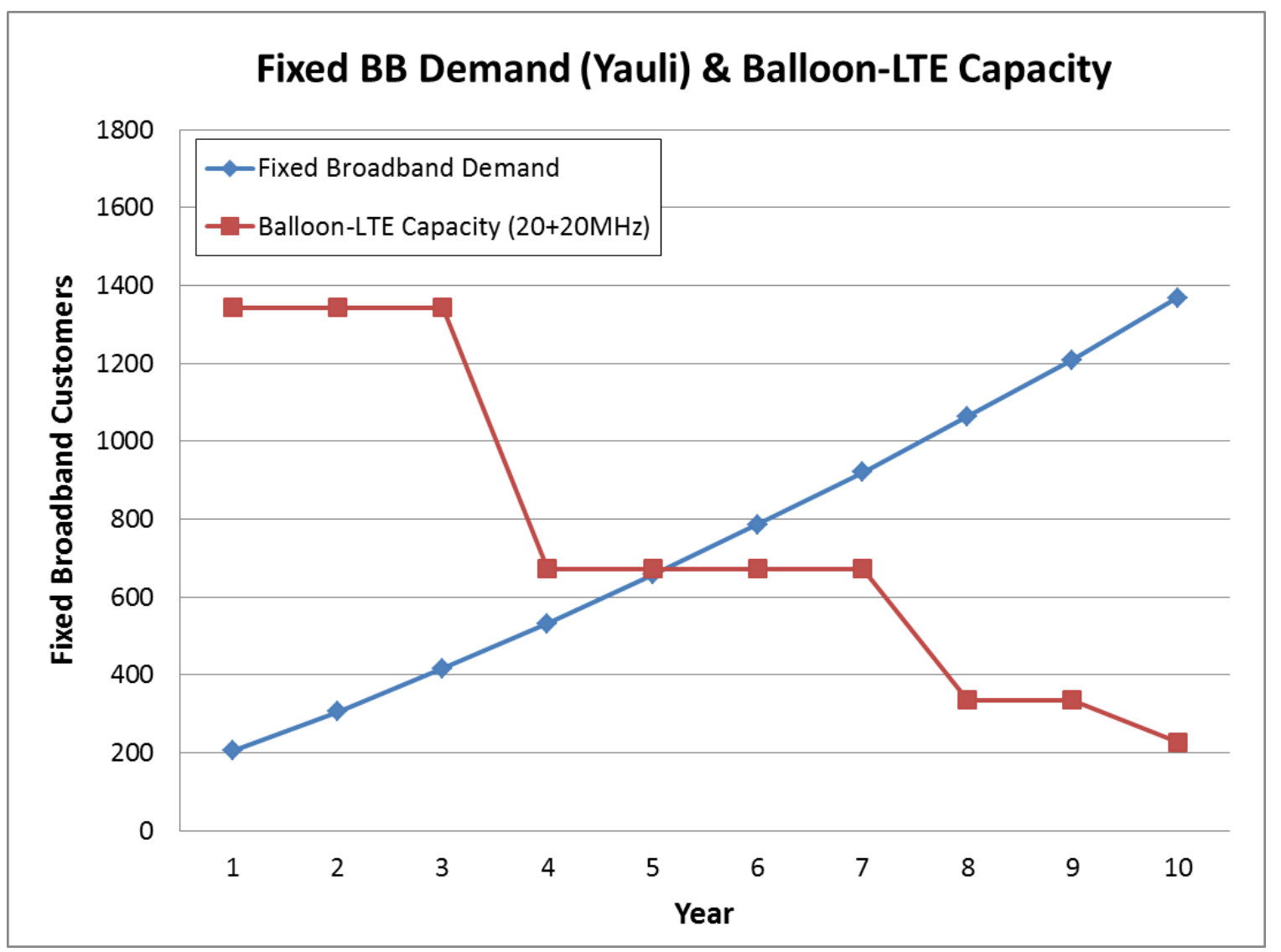

Figure 25. Fixed broadband demand and micro-LTE BS capacity for $20+20 \mathrm{MHz}$ spectrum in Yauli using one balloon.

Figure 26 shows the fixed broadband demand and the balloon's LTE capacity in the Samugari area using two balloons with $20 \mathrm{MHz}$ download spectrum and three sectors for each balloon. In this case, the customer and speed demands can be served for seven years. 


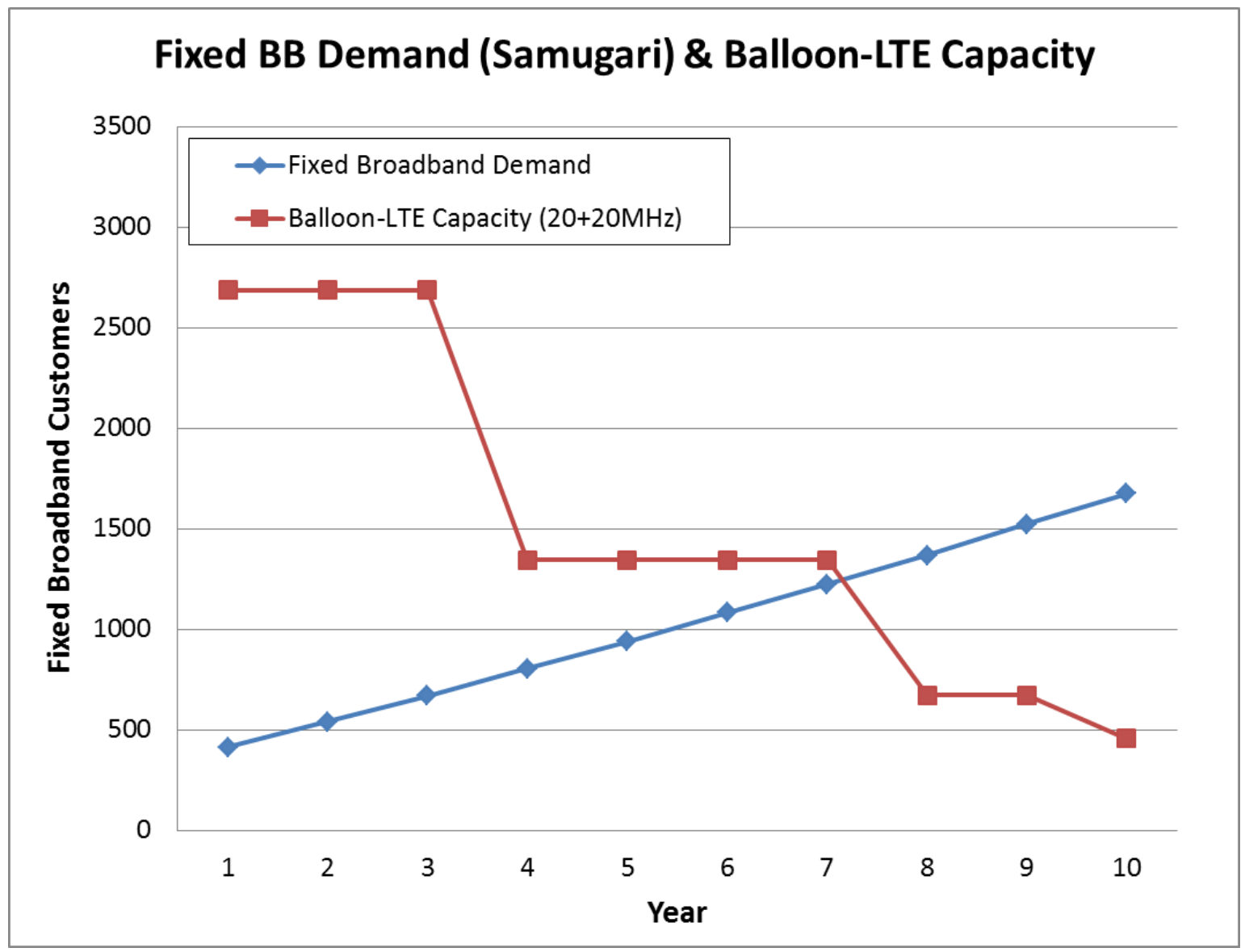

Figure 26. Fixed broadband demand and micro-LTE BS capacity for $20+20 \mathrm{MHz}$ spectrum in Samugari using two balloons.

Table 6 and Table 7 show the network elements required for the stratospheric WBLAN using balloons and micro-LTE base stations for Yauli and Samugari, respectively. Using $20 \mathrm{MHz}$ LTE download channel and up to three sectors allow providing broadband service for five years in Yauli (results shown in Y1 and Y4) and for seven years in Samugari (results shown in Y1 and Y4). 
Table 6. WBLAN design results for Yauli for stratospheric balloons using 20MHz download spectrum.

\begin{tabular}{|l|c|c|c|c|}
\cline { 2 - 5 } \multicolumn{1}{c|}{} & \multicolumn{4}{c|}{$\begin{array}{c}\text { Stratospheric balloons using } 20 \mathrm{MHz} \\
\text { download spectrum in Yauli }\end{array}$} \\
\cline { 2 - 5 } \multicolumn{1}{c|}{} & $\begin{array}{c}\text { Y1 } \\
(2 \mathrm{Mbps})\end{array}$ & $\begin{array}{c}\text { Y4 } \\
(4 \mathrm{Mbps})\end{array}$ & $\begin{array}{c}\text { Y8 } \\
(8 \mathrm{Mbps})\end{array}$ & $\begin{array}{c}\text { Y10 } \\
(12 \mathrm{Mbps})\end{array}$ \\
\hline Nodes & 1 & 1 & - & - \\
\hline Towers & 1 & 1 & - & - \\
\hline Base Stations/Access Points & 2 & 6 & - & - \\
\hline Backhaul Radio Links & 2 & 2 & - & - \\
\hline CPEs & 205 & 532 & - & - \\
\hline
\end{tabular}

Table 7. WBLAN design results for Samugari for stratospheric balloons using $20 \mathrm{MHz}$ download spectrum.

\begin{tabular}{|l|c|c|c|c|}
\cline { 2 - 5 } \multicolumn{1}{c|}{} & \multicolumn{4}{c|}{$\begin{array}{c}\text { Stratospheric balloons using } 20 \mathrm{MHz} \\
\text { download spectrum in Samugari }\end{array}$} \\
\cline { 2 - 5 } \multicolumn{1}{c|}{} & $\begin{array}{c}\text { Y1 } \\
\text { (2Mbs) }\end{array}$ & $\begin{array}{c}\text { Y4 } \\
(4 \mathrm{Mbps})\end{array}$ & $\begin{array}{c}\text { Y8 } \\
(8 \mathrm{Mbps})\end{array}$ & $\begin{array}{c}\text { Y10 } \\
(12 \mathrm{Mbps})\end{array}$ \\
\hline Nodes & 1 & 1 & - & - \\
\hline Towers & 1 & 1 & - & - \\
\hline Base Stations/Access Points & 4 & 8 & - & - \\
\hline Backhaul Radio Links & 4 & 4 & - & - \\
\hline CPE & 413 & 803 & - & - \\
\hline
\end{tabular}

In Chapter 5 Broadband Technology Roadmap, an optimal solution of combining stratospheric balloons and terrestrial networks over the 10-year period is presented. The technical and engineering cost analyses results of this proposed solution are also presented. 


\subsection{WBLAN Engineering cost analysis results - Yauli (Andes Region) and Samugari (Amazon Region) study areas}

\subsubsection{Current Technologies: Wi-Fi, WiMAX, LTE and TVWS Total Cumulative Deployment and Operation Cost (CAPEX and OPEX)}

This total cost includes the combined cumulative CAPEX and OPEX for the deployment and operation costs of the WBLANs for the 10-year period. Figure 27 shows total cost over 10 years for the Yauli area for the Wi-Fi, WiMAX, TVWS and LTE technologies, and also for the stratospheric balloons using LTE. After the initial deployment (Y1), the WBLANs require additional investment in the 10-year period due to, first, upgrades in network capacity to serve growing customer and speed demands, and second, operation and maintenance of the network. The network capacity upgrades due to the growing customer demand is reflected in the total cumulative cost as incremental annual increases, which in most cases is due to the addition of only CPEs. On the other hand, network capacity upgrades due to increase in the speed demand (2Mbps, $4 \mathrm{Mbps}, 8 \mathrm{Mbps}$ and $12 \mathrm{Mbps})$ is reflected in the total cumulative cost as larger increases (steeper slopes) in years 4, 8 and 10 . These capacity upgrades in most cases are due to deployment of additional nodes which includes towers, civil engineering, energy system, and network equipment (AP/BSs and backhauls).

Figure 27 shows that the stratospheric balloons (using LTE in the payload), both single balloon or balloon constellation configurations, are the lowest cost 
options at deployment and for the first three years of operation, however, based on current LTE spectrum regulations and technical co-channel interference constraints, stratospheric balloons would only be able to serve the growing customer and speed demand for the first five years. On the other hand, terrestrial WBLANs for the analyzed technologies (Wi-Fi, WiMAX, LTE and TVWS) are able to handle the customer and speed demand for the 10-year period. Figure 27 shows that from year 1 to year 3 TVWS has the lowest cost, followed by LTE and WiMAX, and Wi-Fi has the highest cost. In these years, TVWS requires less number of nodes due to greater coverage of lower frequency operation. From year 4 to year 7 , LTE becomes the lowest cost option and TVWS becomes the highest, while Wi-Fi and WiMAX have costs close to each other. From year 8 to year 10, TVWS continues having the highest cost, followed by WiMAX. In these last three years, LTE remains the lowest cost option, followed by Wi-Fi.

From years 4 to 7 , there is no significant cost increase or steeper slopes due to not reaching the maximum node capacity (users and Mbps), so there was no need of additional infrastructure or nodes. 


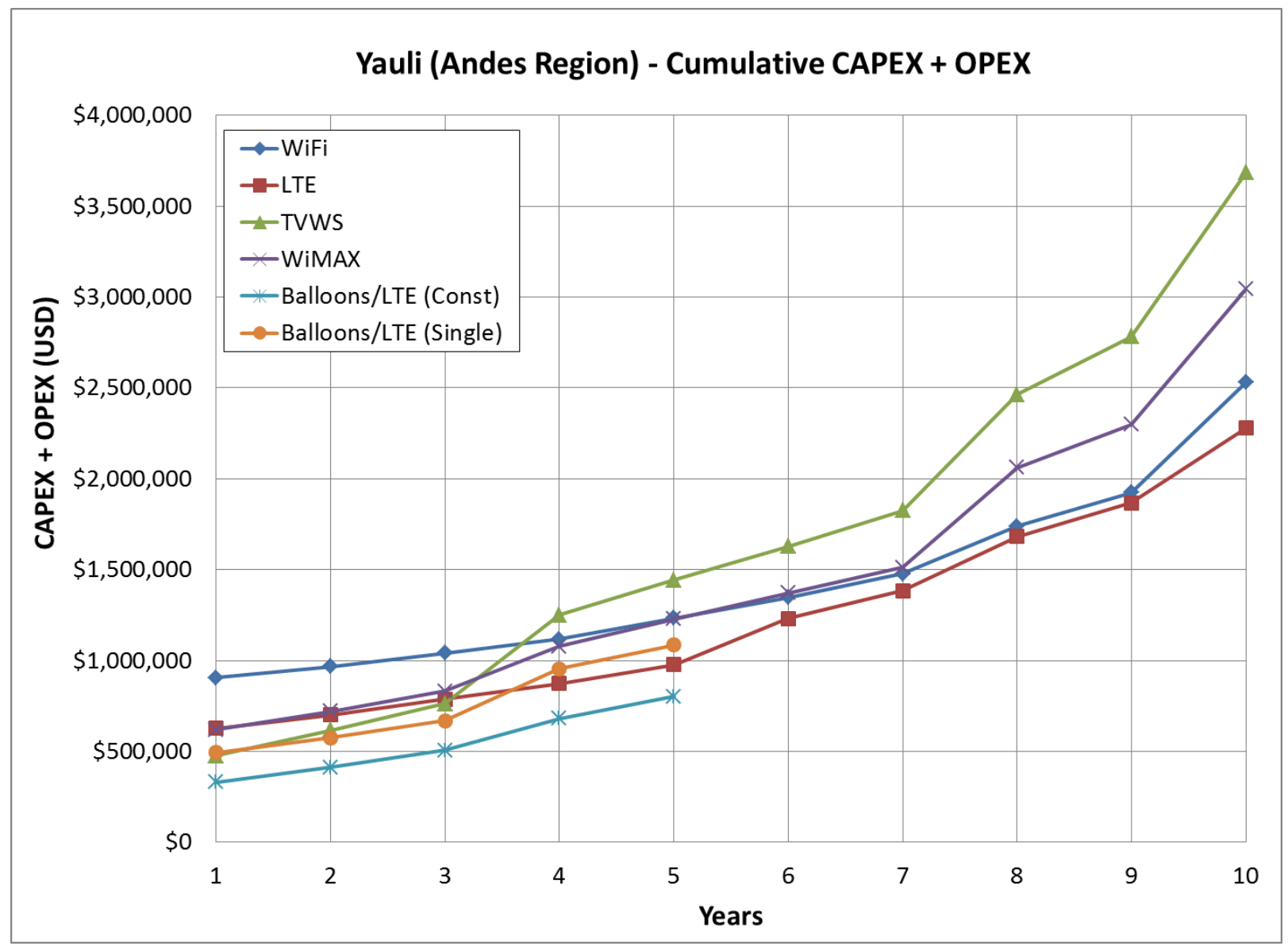

Figure 27. Total cumulative CAPEX and OPEX for the deployment and operation of the WBLANs for the 10-year period in Yauli.

Figure 28 shows the total cumulative CAPEX and OPEX for the Samugari area. This figure shows the stratospheric balloons (using LTE to provide broadband service), both single balloon or balloon constellation configurations, are the lowest cost options at deployment and for seven years of operation; however, stratospheric balloons would only be able to serve the growing customer and speed demand for seven years. Among the terrestrial technology options, in year 1 at deployment, TVWS has the lowest initial cost, followed by LTE and WiMAX, while Wi-Fi has the highest cost. From year 2 to year 3, LTE becomes the lowest cost option closely followed by TVWS. From year 4 to year 7, LTE remains the lowest cost option, 
followed by TVWS and WIMAX, which have similar costs. Wi-Fi remains the highest cost option. From year 8 to year 10, TVWS becomes the highest cost, followed by WiMAX and then Wi-Fi, while LTE ends up as the lowest cost option.

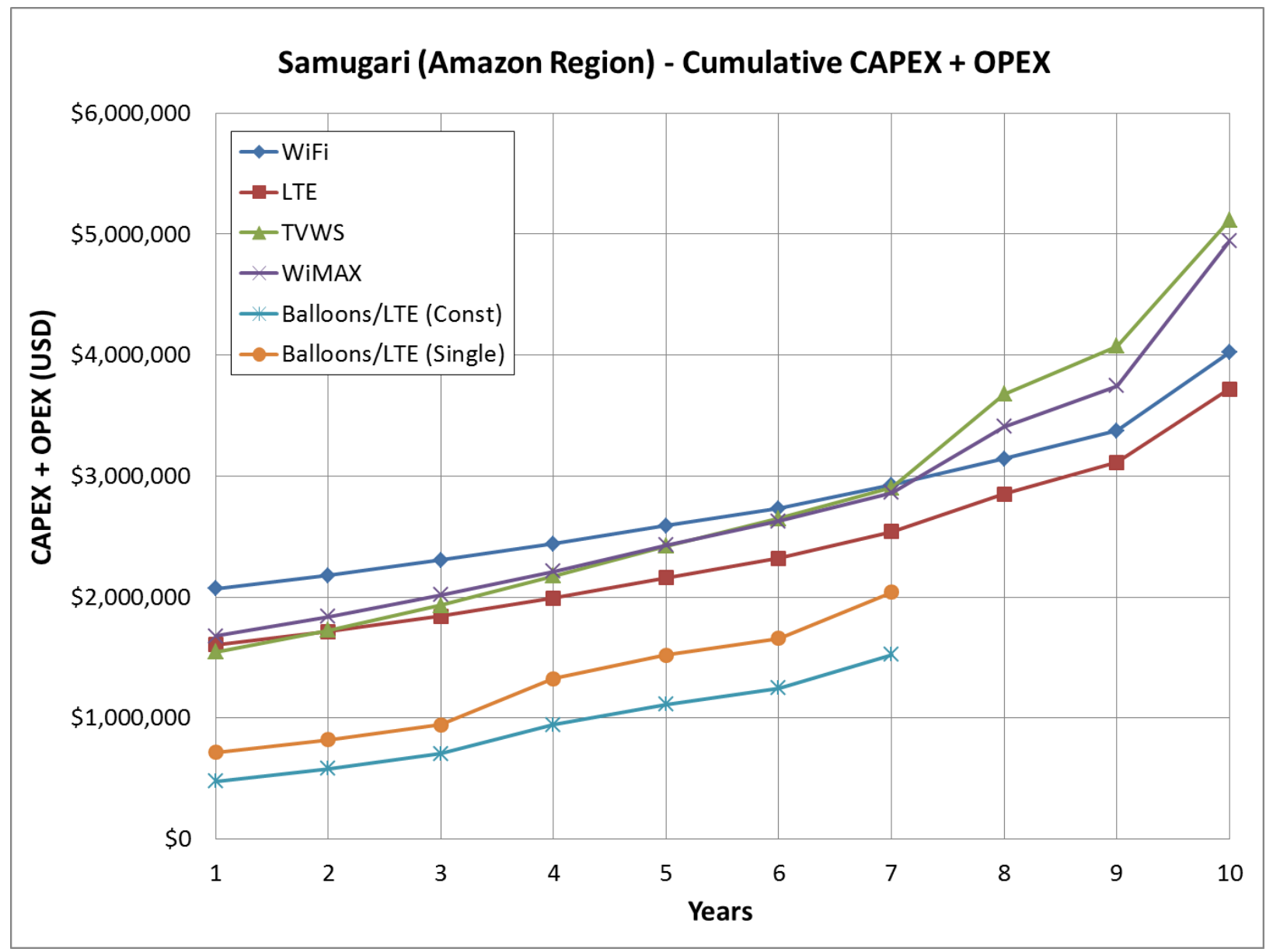

Figure 28. Total cumulative CAPEX and OPEX for the deployment and operation of the WBLANs for the 10-year period in Samugari.

\section{Net Present Value (NPV) of the Total Deployment and Operation Cost (CAPEX and OPEX)}

The NPV allows comparing the present value of the cost of deploying and operating (including both CAPEX and OPEX) the WBLANs over the 10-year period. Figure 29 shows the NPV of the total deployment and operation cost for the 
Yauli and Samugari study areas. The first point to highlight is that the NPV in Samugari is higher than in Yauli for all technologies. In Samugari, the NPV for Wi-Fi, WiMAX, TVWS and LTE is $72 \%, 71 \%, 48 \%$ and $74 \%$ more expensive than in Yauli, respectively. Even when the WBLANs served similar number of towns in both areas, 27 in Yauli and 29 in Samugari, longer distances and dense vegetation between towns in Samugari led to using more nodes in this area, in addition to also using taller towers to enable line-of-sight for the backhauls above the jungle canopy. As a result, WBLAN in Samugari require more expensive infrastructure cost.

In Yauli, LTE has the lowest NPV; followed by Wi-Fi, and then WiMAX and TVWS. The NPV of Wi-Fi, WiMAX and TVWS is 13\%, 29\% and 53\% more expensive than LTE, respectively. Figure 29 also shows the two main components of the NPV: CAPEX and OPEX. For the four technologies, the CAPEX accounts for 61\% (Wi-Fi and LTE) to 67\% (TVWS) of the total NPV, making CAPEX the main cost driver. The CAPEX includes the cost of the towers, civil engineering (node construction), energy system, network equipment and CPE. The OPEX includes technical headquarters operation, network node electric bills, personnel, network operation and authorization fees and fiber optic interconnection fees.

In Samugari, LTE has also the lowest NPV; followed by WiFi, and then WiMAX and TVWS. The NPV of Wi-Fi, WiMAX and TVWS is $12 \%, 27 \%$ and $30 \%$ more expensive than LTE, respectively. In this study area, the CAPEX accounts for $66 \%$ (LTE) to 69\% (TVWS) of the total NPV, making CAPEX the main cost driver. 


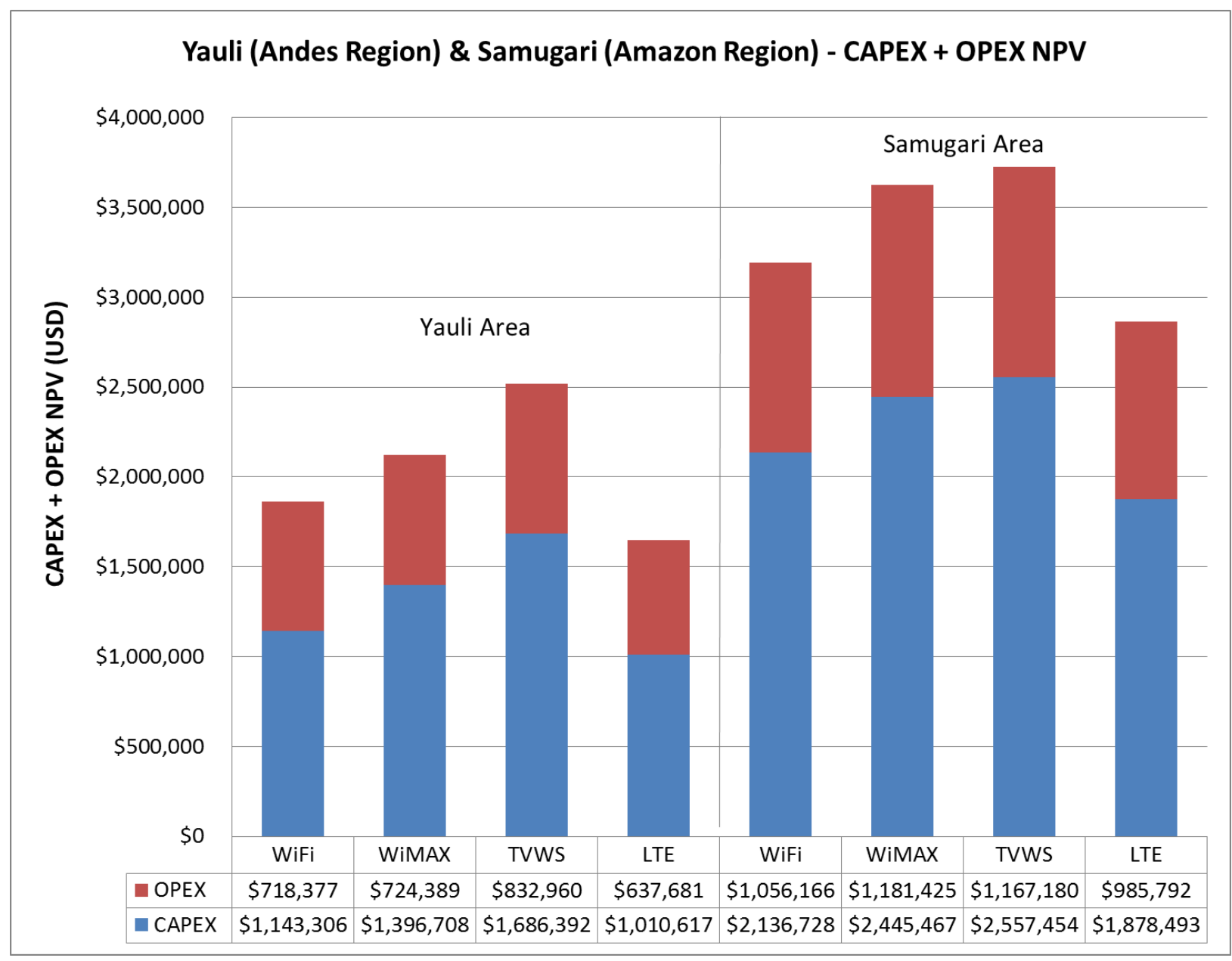

Figure 29. CAPEX and OPEX NPV of WBLANs in Yauli and Samugari.

\section{CAPEX NPV breakdown}

Figure 30 shows the NPV breakdown of the CAPEX in Yauli by cost category, which helps to identify the main cost drivers for the CAPEX for each of the technologies. This breakdown shows the combined cost efficiencies that each of the technologies presents due to their technical features, operation frequency and resulting capacity and coverage. In the Yauli County study area, LTE presents the lowest total CAPEX (USD 1,010K), followed by Wi-Fi (USD 1,143K), and at the higher end WiMAX (USD 1,396K) and TVWS (USD 1,686K). In the aggregated 
categories of towers, civil engineering and energy system (network node infrastructure cost), LTE has the lowest cost (USD 511K), followed by WiMAX (USD 762K), and then TVWS (USD 804K) and Wi-Fi (951K). LTE requires the lowest number of nodes due to providing the most capacity (users and Mbps) per node (see Figure 21), resulting in the lowest infrastructure cost. In the network equipment category (point-to-point backhaul and AP/BSs), Wi-Fi presents the lowest cost (USD 108K), followed by WiMAX (USD 269K), and then TVWS (USD 314K) and LTE (USD 323K). In the CPE category, Wi-Fi also presents the lowest cost (USD 83K), closely followed by LTE (USD 176K), and at the higher end WiMAX (USD 364K) and TVWS (USD 567K). Finally, combining the network equipment and $\mathrm{CPE}$ categories to compare only RF equipment cost of the analyzed technologies, Wi-Fi has the overall lowest cost (USD 191K) and TVWS the highest (USD 882K). Wi-Fi equipment has the RF equipment lowest cost (LTE, WiMAX and TVWS are $161 \%, 231 \%$ and $361 \%$ more expensive than Wi-Fi) due to economies of scale achieved by the Wi-Fi technology; however, a limited capacity of this technology to handle hundreds of CPEs at few kilometers distances, in comparison to the other technologies, results in requiring a higher number of nodes to provide the same coverage. As a result, Wi-Fi infrastructure cost is $18 \%, 25 \%$ and $86 \%$ more expensive than TVWS, WiMAX, and LTE, respectively. 


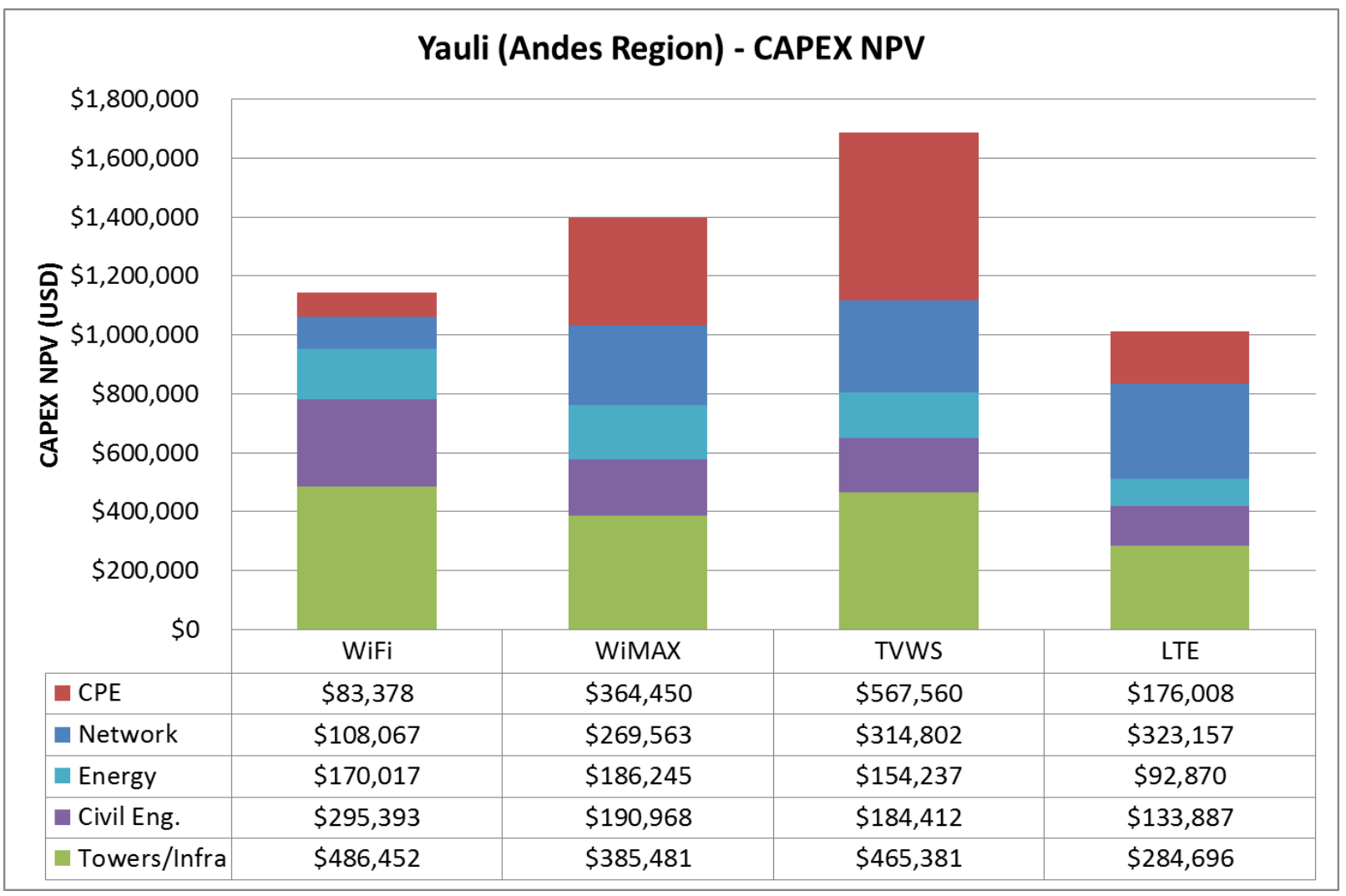

Figure 30. Yauli's CAPEX NPV breakdown in the main categories: energy system, civil engineering, towers, CPE and network equipment.

Figure 31 shows the NPV breakdown of the CAPEX in Samugari by cost category. In the Samugari, LTE presents the lowest total CAPEX (USD 1,878K), followed by Wi-Fi (USD 2,136K) and WiMAX (USD 2,445K), and at the higher end TVWS (USD 2,557K). In the aggregated categories of towers, civil engineering and energy system (network node infrastructure cost), LTE has the lowest cost (USD 1,152K), followed by TVWS (USD 1,372K), and then WiMAX (USD 1,537K) and Wi-Fi (USD 1,847K). LTE requires the lowest number of nodes, 12, due to having the highest capacity per node, followed by TVWS and WiMAX, 17 and 18 nodes respectively. In the network equipment category (point-to-point backhaul and AP/BSs), Wi-Fi presents the lowest cost (USD 178K), followed by TVWS (USD 
409K), WiMAX (USD 411K) and LTE (USD 488K). In the CPE category, Wi-Fi also presents the lowest cost (111K), followed by LTE $(237 \mathrm{~K}))$, and at the higher end WiMAX (USD 496K) and TVWS (USD 775K). Combining the network equipment and CPE categories, Wi-Fi has the lowest combined cost (USD 289K) and TVWS the highest (USD 1,185K). Wi-Fi equipment has the lowest cost (LTE, WiMAX and TVWS are $151 \%, 214 \%$ and $310 \%$ more expensive than Wi-Fi) due to economies of scale achieved by the Wi-Fi technology; however, a limited capacity of this technology to handle hundreds of CPEs at few kilometers distances, in comparison to the other technologies results in requiring a higher number of nodes to provide the same coverage. As a result, Wi-Fi infrastructure cost is $20 \%, 35 \%$ and $60 \%$ more expensive than WiMAX, TVWS and LTE, respectively. 


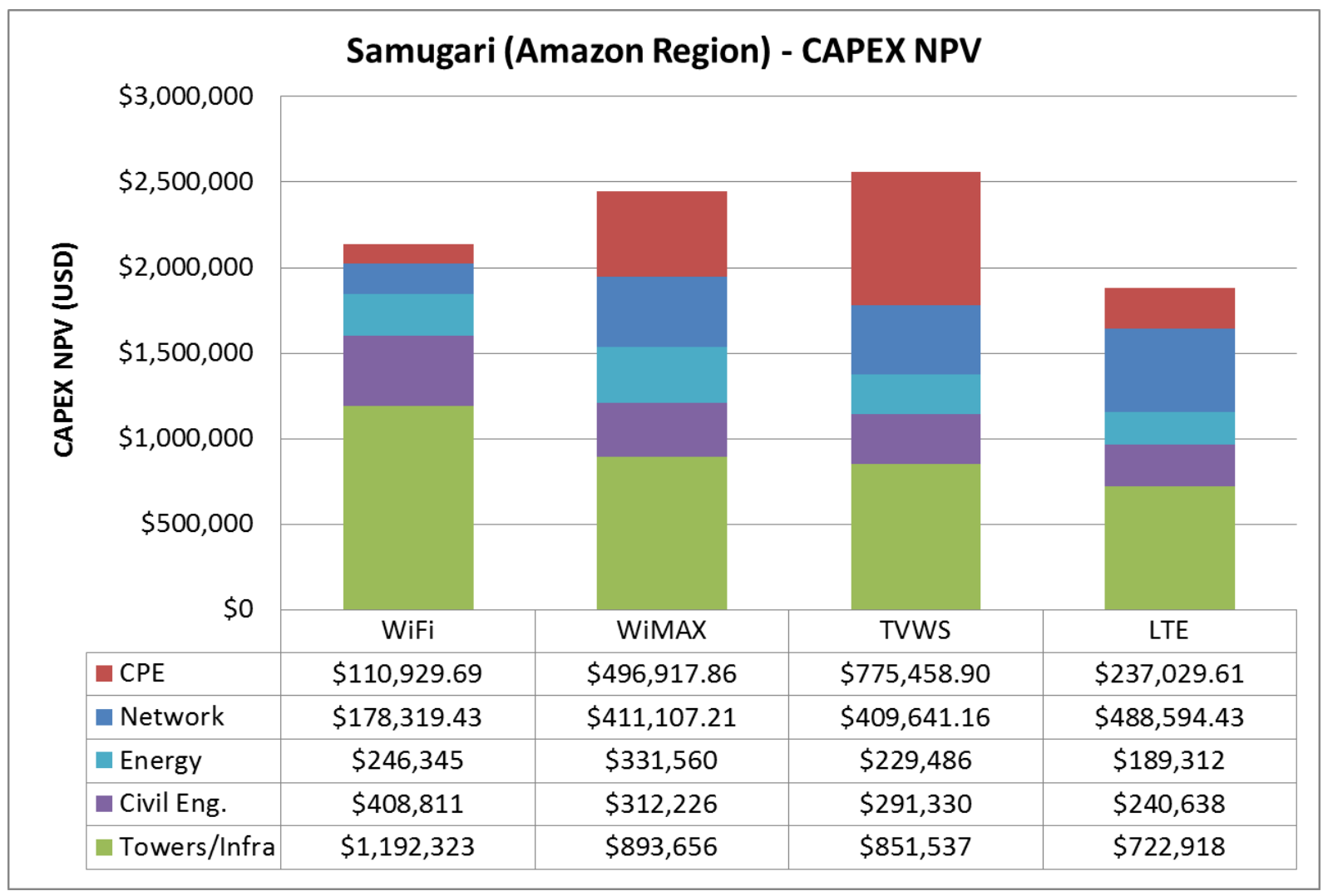

Figure 31. Samugari's CAPEX NPV breakdown in the main categories: energy system, civil engineering, towers, CPE and network equipment.

Figure 32 shows the OPEX NPV breakdown for the Yauli area. OPEX includes headquarters operation, network nodes electricity, engineering and technical personnel, network operation and authorization fees, and fiber optic interconnection. This figure shows LTE has the lowest OPEX cost (USD 637K), followed by Wi-Fi (USD 718K) and WiMAX (USD 724K), and TVWS has the highest (USD 832K). An important point to highlight is that the engineering and technical personnel category accounts for $44 \%$ (WiMAX) to $54 \%$ (TVWS) of the OPEX, making this category the main cost driver. Based on the cost model developed for this study, the personnel ( $\mathrm{RF}$ and networking engineers and technical support) cost depends on the number of nodes, AP/BSs and CPEs. 
Technologies that require more nodes and AP/BSs have higher personnel cost. The second cost driver is headquarters operations, which accounts for 27\% (TVWS) to $33 \%$ (LTE) of the OPEX. The headquarters operation cost depends on the size of the network and personnel. Technologies using more nodes have higher HQ operation costs. Another important cost driver is the fiber optic backbone interconnection fees, which are similar for all technologies due to depending on the estimated traffic generated by the customer and speed demand (same for all technologies). The two smaller contributors to the OPEX are nodes electricity cost, which depends on energy consumption and the number of AP/BSs installed in the network, and broadband operator authorization fees.

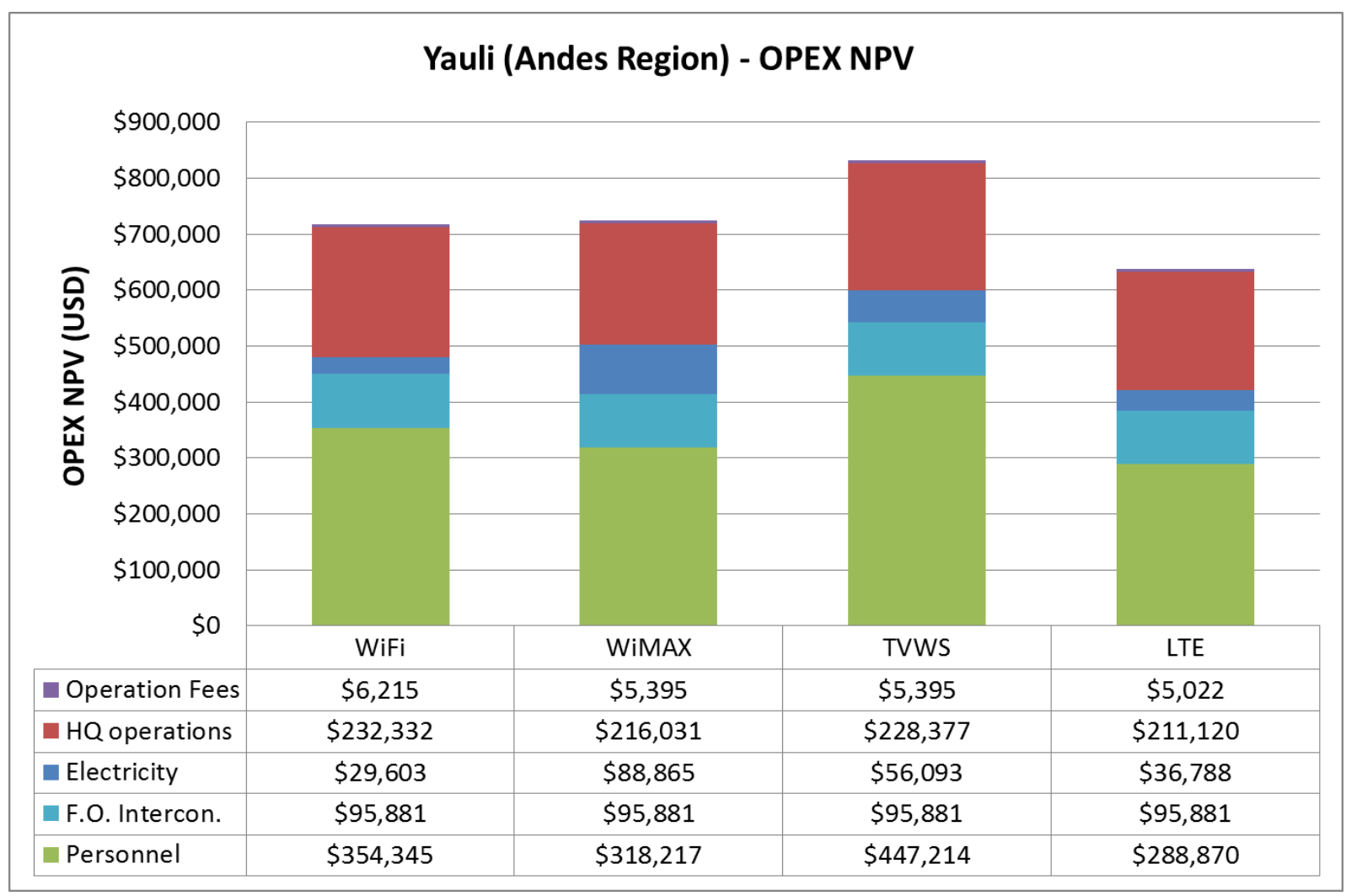

Figure 32. Yauli's OPEX NPV breakdown in the main categories: HQ operation, network electricity, personnel, operation fees and F.O. interconnection. 
Figure 33 shows the OPEX NPV breakdown for the Samugari area. This figure shows LTE has the lowest OPEX cost (USD 985K) and WiMAX has the highest (USD 1,181K). In this study area, engineering and technical personnel category accounts for $42 \%$ (LTE and WiMAX) to $55 \%$ (TVWS) of the OPEX, making this category the main OPEX cost driver. The second cost driver is headquarters operations which accounts for $24 \%$ (TVWS) to 32\% (LTE) of the OPEX. Another important cost driver is the fiber optic backbone interconnection fees, which are similar for all technologies. The two smaller contributors to the OPEX are nodes electricity cost, and broadband operator authorization fees.

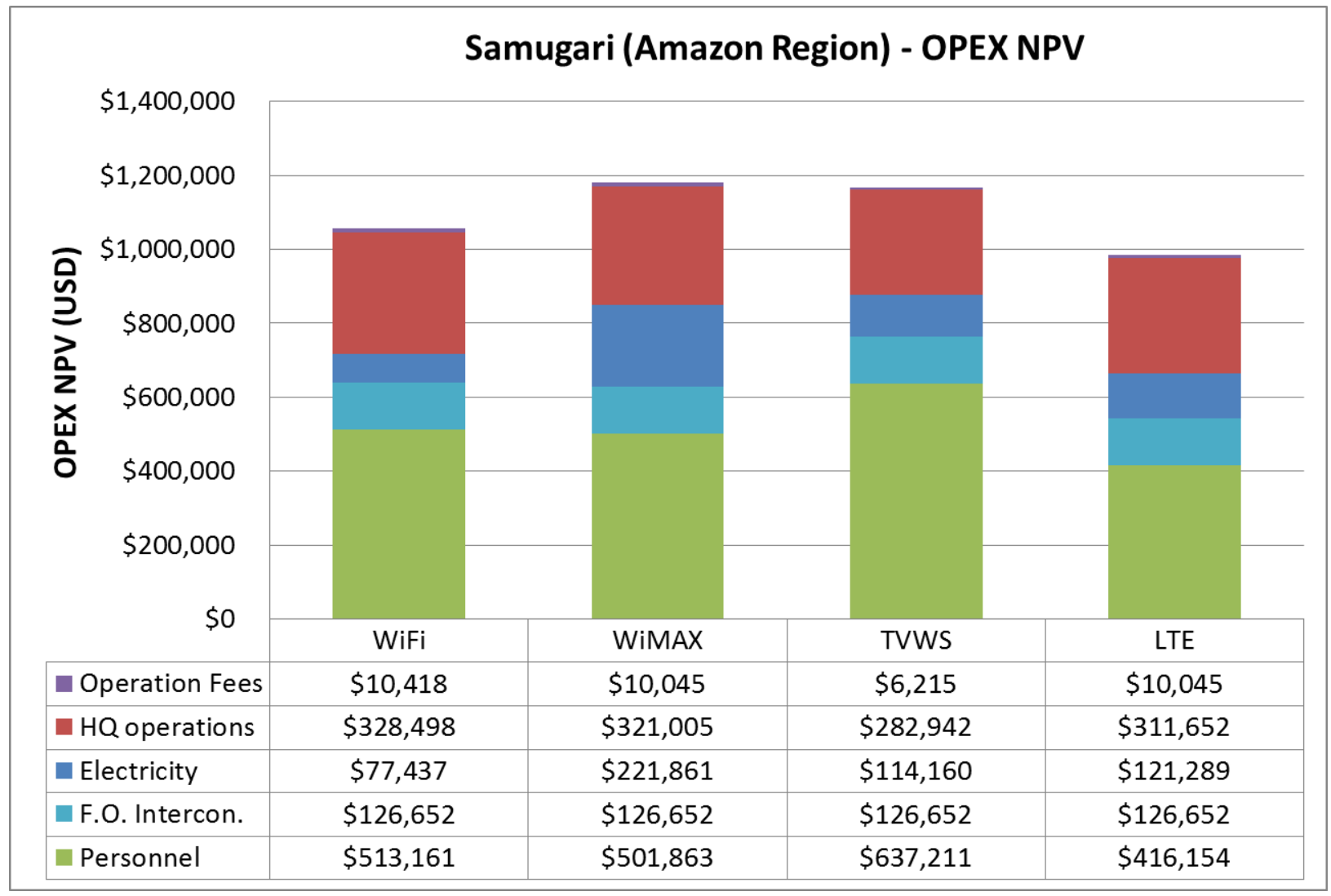

Figure 33. Samugari's OPEX NPV breakdown in the main categories: HQ operation, network electricity, personnel, operation fees and F.O. interconnection. 


\subsubsection{Broadband service cost for the Yauli and Samugari area}

This subsection presents cost metrics oriented to the broadband service provider business in the two study areas: cost per user per month and cost per Mbps per month. For the calculation of these cost metrics, the CAPEX and OPEX per year were used. For the CAPEX per year, it was assumed a standard operator business practice which is annuitizing the CAPEX over a number of years; this is mainly due to using commercial bank loans for the CAPEX and then paying these loans over a number of years. Then for the CAPEX and OPEX calculation per year, it was assumed that the CAPEX per year will be annuitized for a 10-year period. For the calculation of the annuity factor, the current business interest rate in Peru in $2016,6.45 \%$, was used. The calculated annuity factor was 0.138 . The OPEX is not annuitized and assumes its entire yearly cost. Then the cost per user per month is calculated dividing the yearly OPEX and annuitized CAPEX by the number of customers per year and twelve months per year. The cost per Mbps per month is calculated by dividing this cost per user per month by the average broadband speed provided per customer.

\section{Cost per user per month}

The cost per user per month metric is used to estimate the deployment and operation cost per month of the WBLANs per each customer or household to which the network provides broadband service. The calculation for this cost metric was explained in the previous paragraph. The network design and engineering cost analysis were based on our broadband demand and annual demand growth 
estimation (see Section 3.1 Broadband Service Demand Estimation). The initial customer demand for fixed wireless broadband service in the Yauli County is $4.7 \%$ of the households with $2.7 \%$ annual growth; that is, the WBLANs provide fixed broadband service in 27 towns to 205 customers at deployment in year 1, and to 1532 customers at the end of the 10-year period. In the Samugari County, the customer demand is $9.1 \%$ of the households with $2.9 \%$ annual growth; that is the WBLANs provide fixed broadband service in 29 towns to 413 customers at deployment in year 1, and to 1675 customers at the end of the 10-year period. Regarding average broadband speed demand, the initial speed demand is $2 \mathrm{Mbps}$ (Y1-Y3), increasing to 4Mbps (Y4-Y7), 8Mbps (Y8-Y9) and 12Mbps (Y10).

Figure 34 shows the cost per user per month for the Yauli area. It can be noted that for most technologies there is an increase in the cost per user in the years when the speed demand increases (years 4,8 and 10), which leads to additional network investments in capacity. On the other hand, in years when the speed demand is the same, there is a decreasing trend in the cost per user. This figure shows that in the first five years, stratospheric balloons (constellation configuration) have the lowest cost per user. However, as mentioned in the previous section, balloons can only handle the customer and speed demand for the first five years. Regarding the terrestrial technologies, TVWS has the lowest cost per user for the first three years, while LTE and WiMAX have cost per user close to each other, and Wi-Fi has the highest cost. In years 4 to 7, LTE becomes the lowest cost option, followed by WiMAX and Wi-Fi, and TVWS becomes the highest cost 
option. In the last 3 years (years 8, 9 and 10), LTE remains the lowest cost option, followed by Wi-Fi. TVW becomes the highest cost option, followed by WiMAX.

Applying the NPV calculation to the cost per user per month for the 10-year period, LTE is the lowest cost option, USD 207, followed by WiMAX, USD 230, and then TVWS, USD 242, and Wi-Fi, USD 252.

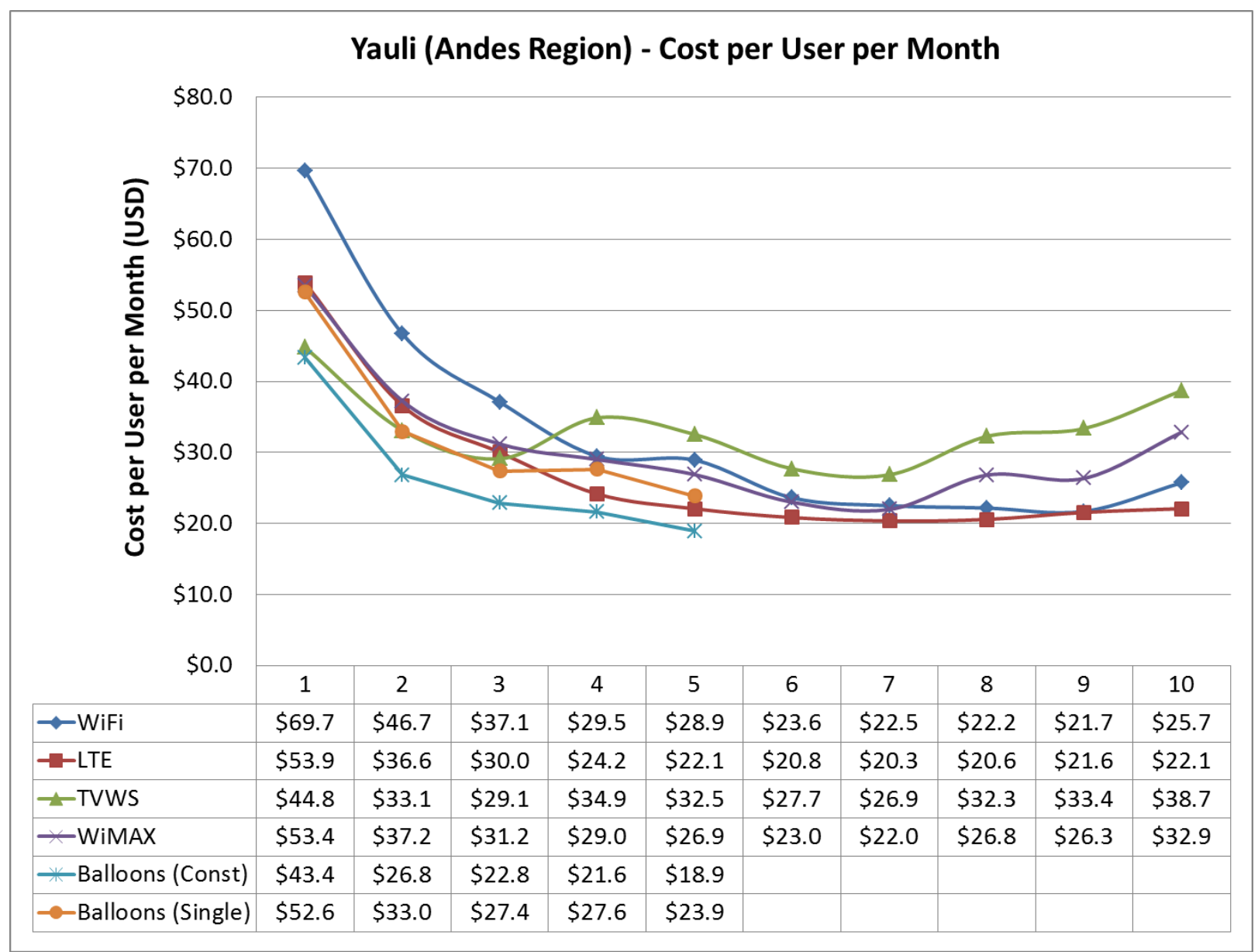

Figure 34. Cost per user per month for the Yauli County study area

Figure 35 shows the cost per user per month for the Samugari area. In the first seven years, stratospheric balloons (both single and constellation configurations) have the lowest cost per user. However, in Samugari, balloons can 
also only handle the customer and speed demand for seven years. Regarding the terrestrial technologies, TVWS and LTE have the lowest cost per user for the first three years, followed by WiMAX, and Wi-Fi has the highest cost. In years 4 to 7 , LTE becomes the lowest cost option, followed by WiMAX, TVWS and Wi-Fi. In the last 3 years (years 8, 9 and 10), LTE remains the lowest cost option, followed by Wi-Fi and WiMAX, and TVWS becomes the highest cost option.

Applying the NPV calculation to the cost per user per month for the 10-year period, LTE is the lowest cost option, USD 263, followed by TVWS, USD 293, and WiMAX, USD 299, and Wi-Fi has the highest cost, USD 313.

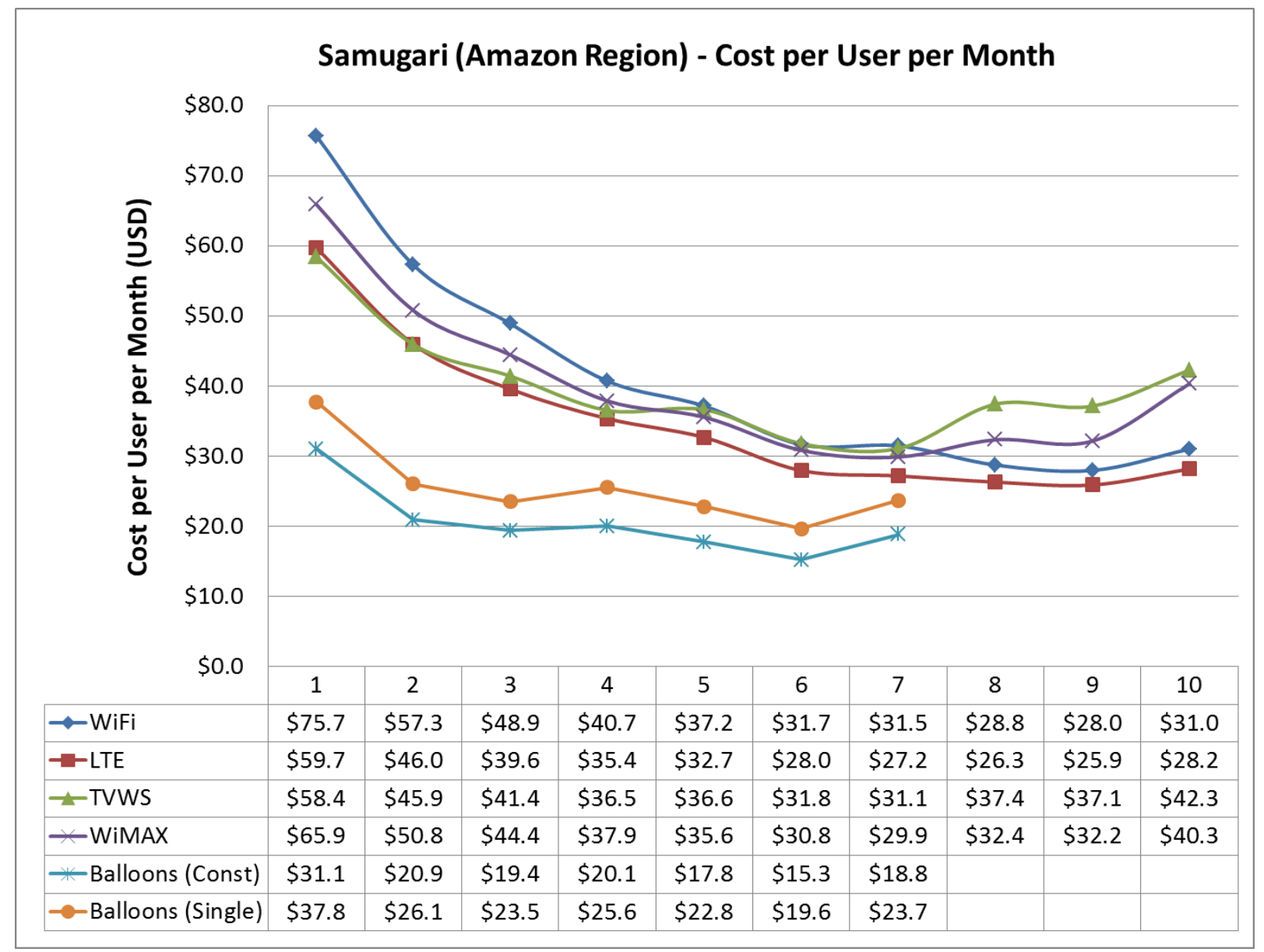

Figure 35. Cost per user per month for the Samugari County study area 


\section{Cost per Mbps per month}

The cost per Mbps per month is used to estimate the deployment and operation cost per month of the WBLANs per Mbps (capacity) that the network provides. This metric is calculated by dividing the cost per user per month by the provided broadband speed per customer. The broadband speeds provided over the 10-year period are the following: $2 \mathrm{Mbps}$ (years 1 to 3), $4 \mathrm{Mbps}$ (years 4 to 7 ), $8 \mathrm{Mbps}$ (years 8 and 9) and 12Mbps (year 10).

Figure 36 shows the cost per Mbps per month in the Yauli County area. It can be noted that for all technologies, there is a decreasing trend in the cost per Mbps in the years. In the first three years, stratospheric balloons (constellation configuration) have the lowest cost per Mbps. However, balloons can only handle the customer and speed demand for five years. Regarding the other technologies, TVWS has the lowest cost per Mbps for the first three years, followed by LTE and WiMAX, while Wi-Fi presents the highest cost. In years 4 to 7, LTE becomes the lowest cost option, closely followed by WiMAX and Wi-Fi, while TVWS becomes the highest cost option. In years 8, 9 and 10, LTE remains the lowest cost option, closely followed by Wi-Fi and then WiMAX and TVWS.

Applying the NPV calculation to the cost per Mbps per month for the 10-year period, LTE is the lowest cost option, USD 74, closely followed by TVWS and WiMAX, USD 76 and USD 78 respectively, and Wi-Fi, USD 92. 


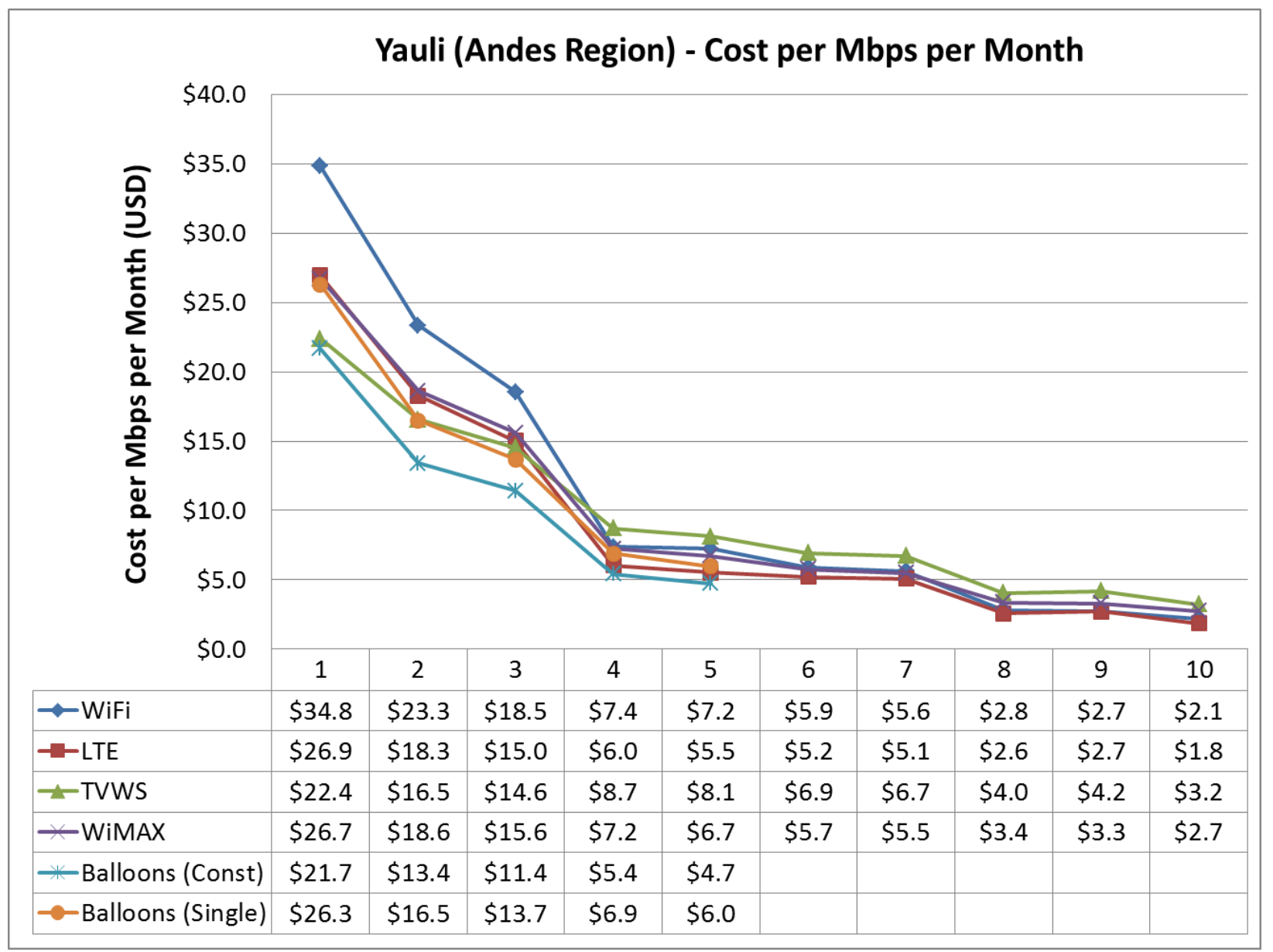

Figure 36. Cost per Mbps per month for the Yauli County study area

Figure 37 shows the cost per Mbps per month in the Samugari County area.

In the first seven years, stratospheric balloons (both single and constellation configurations) have again the lowest cost per Mbps. However, balloons can only handle the customer and speed demand for seven years. Regarding the terrestrial technologies, TVWS and LTE have the lowest cost per Mbps for the first three years, followed by WiMAX, while Wi-Fi presents the highest cost. In years 4 to 7 , LTE becomes the lowest cost option, closely followed by the other three technologies with cost per Mbps close to each other. In years 8, 9 and 10, LTE 
remains the lowest cost option, closely followed by Wi-Fi and WiMAX, while TVWS has the highest cost per Mbps.

Applying the NPV calculation to the cost per Mbps per month for the 10-year period, LTE is the lowest cost option, USD 92, closely followed by TVWS, USD 97, and then WiMAX and Wi-Fi, USD 102 and USD 112, respectively.

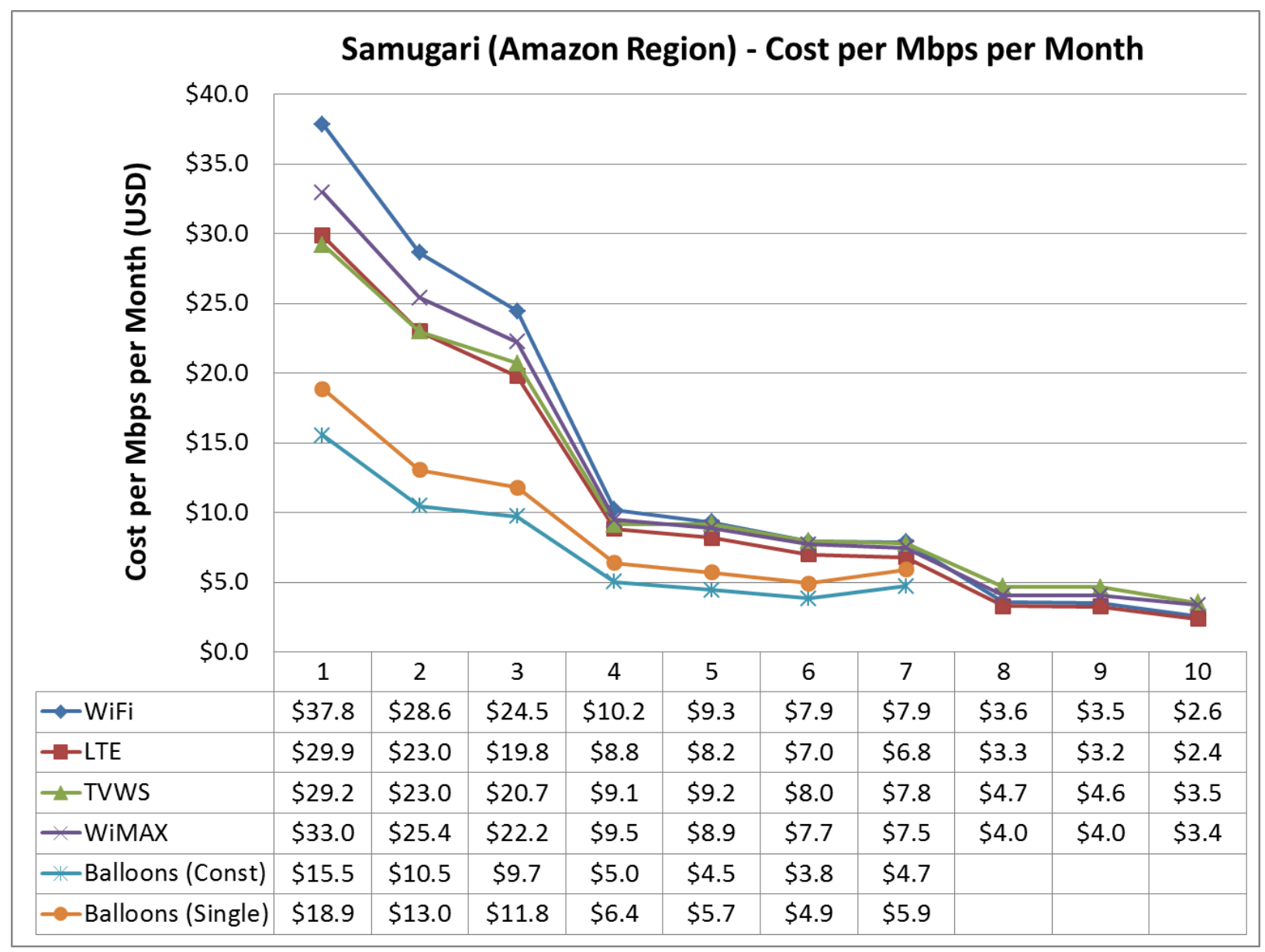

Figure 37. Cost per Mbps per month for the Samugari County study area

\subsection{Sensitivity analysis for current wireless technologies}

The engineering cost analysis in this study includes a sensitivity analysis to determine the impact of cost variations in the net present value (NPV) of the total 
deployment and operation cost of the WBLANs. This subsection presents the results of the sensitivity analysis for cost increases of the following: 1) CAPEX and OPEX categories, 2) wireless APs and BSs, and 3) Infrastructure towers. The sensitivity analysis was conducted using Analytica's specialized tools and functions to calculate the impact on the NPV in response to cost increases in specific variables.

\subsubsection{CAPEX and OPEX categories}

This sensitivity analysis calculates the NPV variation for a $10 \%$ increase in the main CAPEX and OPEX cost categories. The CAPEX categories include the following: network equipment (AP/BSs, backhaul equipment), $\mathrm{CPE}$, towers, civil engineering (node construction), and energy system. The OPEX categories include the following: headquarters operation, network nodes electricity, engineering and technical personnel, network operation and authorization fees, and fiber optic interconnection. Table 8 shows the NPV cost variation percentage for a $10 \%$ cost increase in each CAPEX and OPEX cost category in the Yauli area; one category increase at a time. The table shows, in the CAPEX, that for Wi-Fi and WiMAX, the NPV is more sensitive to a tower cost increase; $10 \%$ increase in the towers cost results in a $2.6 \%$ and $1.8 \%$ total NPV increase in Wi-Fi and WiMAX, respectively. For TVWS, $10 \%$ CPE cost increase has the highest impact of a $2.3 \%$ NPV increase, and for LTE, 10\% network cost increase has the highest impact of a $2 \%$ NPV increase. In the OPEX, for all technologies, $10 \%$ personnel cost increase has the highest impact on the NPV. Table 9 shows the calculated NPV for a $10 \%$ cost 
increase of the CAPEX and OPEX categories. The first column in the table shows that LTE has the lowest initial NPV. The table shows that after a $10 \%$ cost increase in each category, LTE remains the lowest cost option.

Table 8. NPV percentage variation to a $10 \%$ increase in each CAPEX and OPEX main category in Yauli.

\begin{tabular}{|c|c|c|c|c|c|c|c|c|c|c|}
\hline & \multicolumn{10}{|c|}{$\begin{array}{c}\text { Yauli Area - NPV Sensitivity Analysis to a 10\% Cost Increase - Percentage } \\
\text { Variation (\%) }\end{array}$} \\
\hline & \multicolumn{5}{|c|}{ CAPEX } & \multicolumn{5}{|c|}{ OPEX } \\
\hline & $\begin{array}{c}\text { NET- } \\
\text { WORK } \\
\text { COST }\end{array}$ & $\begin{array}{l}\text { CPE } \\
\text { COST }\end{array}$ & $\begin{array}{c}\text { TOWER } \\
\text { COST }\end{array}$ & $\begin{array}{l}\text { CIVIL } \\
\text { ENG. } \\
\text { COST }\end{array}$ & $\begin{array}{c}\text { ENERG } \\
\text { SYST } \\
\text { COST }\end{array}$ & $\begin{array}{c}\text { HQ } \\
\text { OPS } \\
\text { COST }\end{array}$ & $\begin{array}{l}\text { ELECTR } \\
\text { COST }\end{array}$ & $\begin{array}{l}\text { PERSO } \\
\text {-NNEL } \\
\text { COST }\end{array}$ & $\begin{array}{c}\text { OP } \\
\text { FEES } \\
\text { COST }\end{array}$ & $\begin{array}{l}\text { F.O. } \\
\text { INTER } \\
\text { COST }\end{array}$ \\
\hline Wi-Fi & $0.6 \%$ & $0.4 \%$ & $2.6 \%$ & $1.6 \%$ & $0.9 \%$ & $1.2 \%$ & $0.2 \%$ & $1.9 \%$ & $0.03 \%$ & $0.5 \%$ \\
\hline WiMAX & $1.3 \%$ & $1.7 \%$ & $1.8 \%$ & $0.9 \%$ & $0.9 \%$ & $1.0 \%$ & $0.4 \%$ & $1.5 \%$ & $0.03 \%$ & $0.5 \%$ \\
\hline TVWS & $1.2 \%$ & $2.3 \%$ & $1.9 \%$ & $0.7 \%$ & $0.6 \%$ & $0.9 \%$ & $0.2 \%$ & $1.8 \%$ & $0.02 \%$ & $0.4 \%$ \\
\hline LTE & $2.0 \%$ & $1.1 \%$ & $1.7 \%$ & $0.8 \%$ & $0.6 \%$ & $1.3 \%$ & $0.2 \%$ & $1.8 \%$ & $0.03 \%$ & $0.6 \%$ \\
\hline
\end{tabular}

Table 9. Recalculated NPV to a 10\% increase in each CAPEX and OPEX main category in Yauli.

\begin{tabular}{|c|c|c|c|c|c|c|c|c|c|c|c|}
\hline & & \multicolumn{10}{|c|}{ Yauli Area - NPV Sensitivity Analysis to a $10 \%$ Cost Increase - NPV } \\
\hline & \multirow[b]{2}{*}{$\begin{array}{c}\text { Initial } \\
\text { NPV }\end{array}$} & \multicolumn{5}{|c|}{ CAPEX } & \multicolumn{5}{|c|}{ OPEX } \\
\hline & & $\begin{array}{l}\text { NET- } \\
\text { WORK } \\
\text { COST }\end{array}$ & $\begin{array}{c}\text { CPE } \\
\text { COST }\end{array}$ & $\begin{array}{c}\text { TOWER } \\
\text { COST }\end{array}$ & $\begin{array}{l}\text { CIVIL } \\
\text { ENG. } \\
\text { COST }\end{array}$ & $\begin{array}{c}\text { ENERG } \\
\text { SYST } \\
\text { COST }\end{array}$ & $\begin{array}{c}\mathrm{HQ} \\
\mathrm{OPS} \\
\mathrm{COST}\end{array}$ & $\begin{array}{c}\text { ELECT } \\
\text { COST }\end{array}$ & $\begin{array}{l}\text { PERSO } \\
\text {-NNEL } \\
\text { COST }\end{array}$ & $\begin{array}{c}\text { OP } \\
\text { FEES } \\
\text { COST }\end{array}$ & $\begin{array}{c}\text { F.O. } \\
\text { INTER } \\
\text { COST }\end{array}$ \\
\hline Wi-Fi & $1,861 \mathrm{~K}$ & $1,872 \mathrm{~K}$ & $1,870 \mathrm{~K}$ & $1,911 \mathrm{~K}$ & $1,891 \mathrm{~K}$ & $1,878 \mathrm{~K}$ & $1,885 \mathrm{~K}$ & $1,864 \mathrm{~K}$ & $1,897 \mathrm{~K}$ & $1,862 \mathrm{~K}$ & $1,871 \mathrm{~K}$ \\
\hline WiMAX & $2,120 \mathrm{~K}$ & $2,147 \mathrm{~K}$ & $2,157 \mathrm{~K}$ & $2,159 \mathrm{~K}$ & $2,139 \mathrm{~K}$ & $2,139 \mathrm{~K}$ & $2,142 \mathrm{~K}$ & $2,129 \mathrm{~K}$ & $2,152 \mathrm{~K}$ & $2,121 \mathrm{~K}$ & $2,130 \mathrm{~K}$ \\
\hline TVWS & $2,519 \mathrm{~K}$ & $2,550 \mathrm{~K}$ & $2,576 \mathrm{~K}$ & $2,566 \mathrm{~K}$ & $2,537 \mathrm{~K}$ & $2,534 \mathrm{~K}$ & $2,542 \mathrm{~K}$ & $2,524 \mathrm{~K}$ & $2,564 \mathrm{~K}$ & $2,519 \mathrm{~K}$ & $2,528 \mathrm{~K}$ \\
\hline LTE & $1,648 \mathrm{~K}$ & $1,680 \mathrm{~K}$ & $1,665 \mathrm{~K}$ & $1,677 \mathrm{~K}$ & $1,661 \mathrm{~K}$ & $1,657 \mathrm{~K}$ & $1,669 \mathrm{~K}$ & $1,651 \mathrm{~K}$ & $1,667 \mathrm{~K}$ & $1,648 \mathrm{~K}$ & $1,657 \mathrm{~K}$ \\
\hline
\end{tabular}

Table 10 shows the NPV cost variation percentage for a $10 \%$ cost increase in

each CAPEX and OPEX cost category in Samugari; one category increase at a time.

This table shows, in the CAPEX, that for the four technologies, the NPV is more sensitive to a tower cost increase. In the OPEX, for all technologies, 10\% personnel cost increase has the highest impact on the NPV. Table 11 shows the recalculated 
NPV for a $10 \%$ cost increase of the CAPEX and OPEX categories in Samugari. The first column in the table shows that LTE has the lowest initial NPV. The table shows that after a $10 \%$ cost increase in each category, LTE remains the lowest cost option.

Table 10. NPV percentage variation to a $10 \%$ increase in each CAPEX and OPEX main category in Samugari

\begin{tabular}{|l|c|c|c|c|c|c|r|c|c|c|}
\cline { 2 - 11 } & \multicolumn{8}{|c|}{ Samugari Area - NPV Sensitivity Analysis to a 10\% Cost Increase - } \\
Percentage Variation (\%)
\end{tabular}

Table 11. Recalculated NPV to a 10\% increase in each CAPEX and OPEX main category in Samugari

\begin{tabular}{|c|c|c|c|c|c|c|c|c|c|c|c|}
\hline & \multirow[b]{3}{*}{$\begin{array}{c}\text { Initial } \\
\text { NPV }\end{array}$} & \multicolumn{10}{|c|}{ Samugari Area - NPV Sensitivity Analysis to a $10 \%$ Cost Increase - NPV } \\
\hline & & \multicolumn{5}{|c|}{ CAPEX } & \multicolumn{5}{|c|}{ OPEX } \\
\hline & & \begin{tabular}{|c|} 
NET- \\
WORK \\
COST
\end{tabular} & $\begin{array}{c}\mathrm{CPE} \\
\mathrm{COST}\end{array}$ & $\begin{array}{c}\text { TOWER } \\
\text { COST }\end{array}$ & $\begin{array}{l}\text { CIVIL } \\
\text { ENG. } \\
\text { COST }\end{array}$ & $\begin{array}{c}\text { ENERG } \\
\text { SYST } \\
\text { COST }\end{array}$ & $\begin{array}{c}\text { HQ } \\
\text { OPS } \\
\text { COST }\end{array}$ & $\begin{array}{c}\text { ELECTR } \\
\text { COST }\end{array}$ & $\begin{array}{l}\text { PERSO } \\
\text {-NNEL } \\
\text { COST }\end{array}$ & \begin{tabular}{|c} 
OP \\
FEES \\
COST
\end{tabular} & \begin{tabular}{|l} 
F.O. \\
INTER \\
COST
\end{tabular} \\
\hline Wi-Fi & $3,193 \mathrm{~K}$ & $3,211 \mathrm{~K}$ & $3,204 \mathrm{~K}$ & $3,313 \mathrm{~K}$ & $3,234 \mathrm{~K}$ & $3,217 \mathrm{~K}$ & $3,226 \mathrm{~K}$ & $3,201 \mathrm{~K}$ & $3,244 \mathrm{~K}$ & $3,194 \mathrm{~K}$ & $3,205 \mathrm{~K}$ \\
\hline WiMAX & $3,626 \mathrm{~K}$ & $3,667 \mathrm{~K}$ & $3,676 \mathrm{~K}$ & $3,716 \mathrm{~K}$ & $3,657 \mathrm{~K}$ & $3,659 \mathrm{~K}$ & $3,658 \mathrm{~K}$ & $3,648 \mathrm{~K}$ & $3,676 \mathrm{~K}$ & $3,627 \mathrm{~K}$ & $3,639 \mathrm{~K}$ \\
\hline TVWS & $3,724 \mathrm{~K}$ & $3,765 \mathrm{~K}$ & $3,802 \mathrm{~K}$ & $3,810 \mathrm{~K}$ & $753 \mathrm{~K}$ & $47 \mathrm{~K}$ & $3,752 \mathrm{~K}$ & 3,73 & & $3,725 \mathrm{~K}$ & 3,737 \\
\hline LTE & $2.864 \mathrm{~K}$ & $2,913 \mathrm{~K}$ & $2,887 \mathrm{~K}$ & $2,937 \mathrm{~K}$ & $2,888 \mathrm{~K}$ & $2,883 \mathrm{~K}$ & $2,895 \mathrm{~K}$ & $2,876 \mathrm{~K}$ & $2,905 \mathrm{~K}$ & $2,865 \mathrm{~K}$ & $2.876 \mathrm{~K}$ \\
\hline
\end{tabular}

\subsubsection{Wireless AP/BSs}

For the sensitivity analysis, the cost variations of the APs or BSs for the wireless technology options were also analyzed. As mentioned in the previous 
chapter in Section 3.2.3 RF equipment and network simulation settings, this study uses KPIs to benchmark the different technology options. These KPIs included technical features and upfront and operating expenses of the equipment were analyzed, and at the end, specific APs or BSs for each technology were selected for conducting the technical and engineering cost analyses. In this sensitivity analysis, the price ranges for the APs and BSs for each of the technologies are analyzed to estimate their impact on the NPV. In the previous CAPEX and OPEX categories sensitivity analysis, the network equipment (AP/BSs and backhaul equipment) category was analyzed for a 10\% cost increase; however, this second analysis is different to the previous one in that, first, it only analyzes $\mathrm{AP} / \mathrm{BSs}$ cost variation, and second, the cost increase will vary depending on the AP/BSs cost range for each wireless technology. This price range comes from putting together the quotations provided by the equipment vendors for the different technologies. Figure 38 shows the NPV for AP/BS cost increase in the Yauli area. WiMAX and TVWS do not present a NPV variation due to already using equipment in the high end cost of the range. The selected WiMAX and TVWS equipment were more expensive than other options but provided most capacity and performance, and overall lower NPV. WiMAX and TVWS lower cost equipment did not provide enough capacity for the required customer and speed demands. In the case of Wi-Fi and LTE, the selected options were in the mid-range cost and provided the most capacity and performance, and overall lower NPV. Wi-Fi and LTE lower cost equipment also did not provide enough capacity for the required 
customer and speed demands. On the other hand, Wi-Fi and LTE higher cost equipment provided enough capacity and other additional technical features or characteristics for easier remote network management tools. This figure shows the NPV and the NPV cost increase using high end cost equipment for the analyzed technologies. As mentioned above, WiMAX and TVWS were already at the highest end of the cost equipment. In the case of Wi-Fi and LTE, using high end cost equipment, the NPV for Wi-Fi and LTE increases USD 74,767 and USD 523,867, respectively. After this AP/BSs cost increase, Wi-Fi becomes the lowest cost option.

Figure 39 shows the NPV for AP/BS cost increase in the Samugari area. The NPV for Wi-Fi and LTE increases USD 87,577 and USD 740,520K, respectively. After this AP/BSs cost increase, Wi-Fi becomes the lowest cost option.

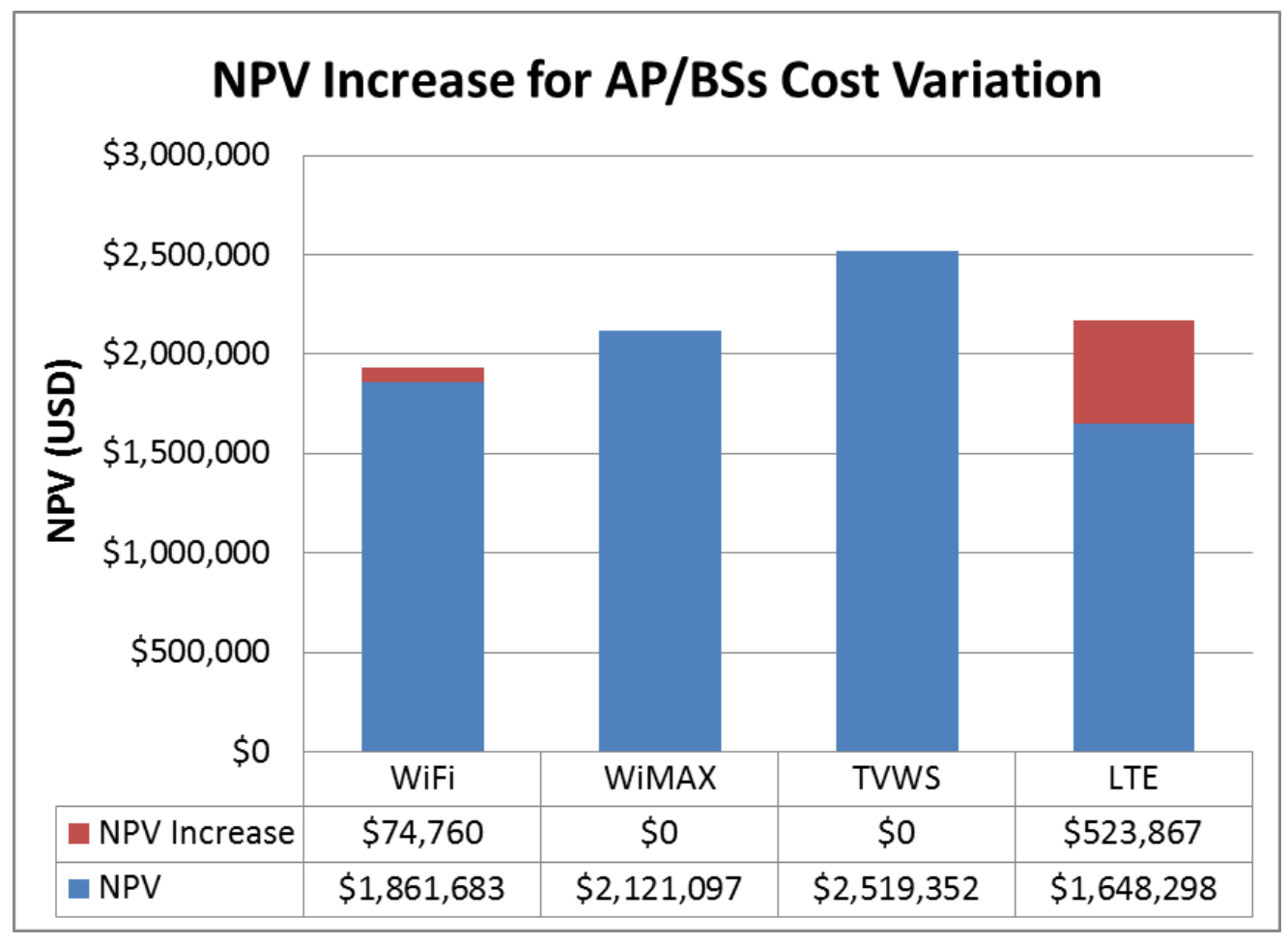

Figure 38. NPV increase for AP/BS cost variation in Yauli 


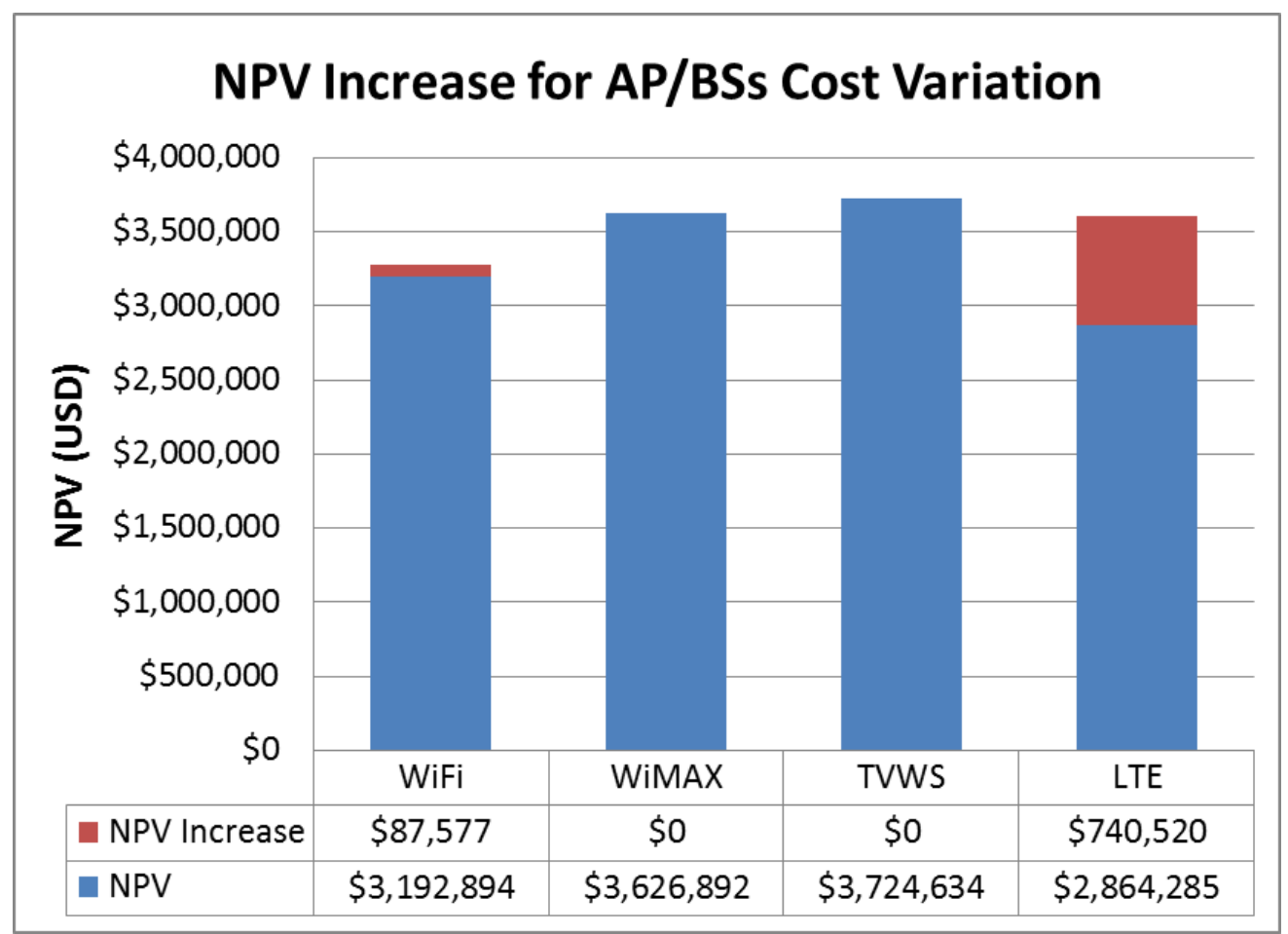

Figure 39. NPV increase for AP/BS cost variation in Samugari

\subsubsection{Tower Infrastructure}

The FITEL requirements for the deployment of the WBLANs include using self-supported telecommunication towers. The cost of this type of towers is around 1.5 to 5 times more expensive than cable-supported towers, depending on the height. Both types of towers are shown in Figure 40. Self-supported towers are more expensive due to requiring wider cross sections than cable-supported towers, and as a result, using more steel for their construction. This sensitivity analysis calculates the cost impact on the NPV of using cable-supported towers. It is expected that the NPV decreased when using these towers. 


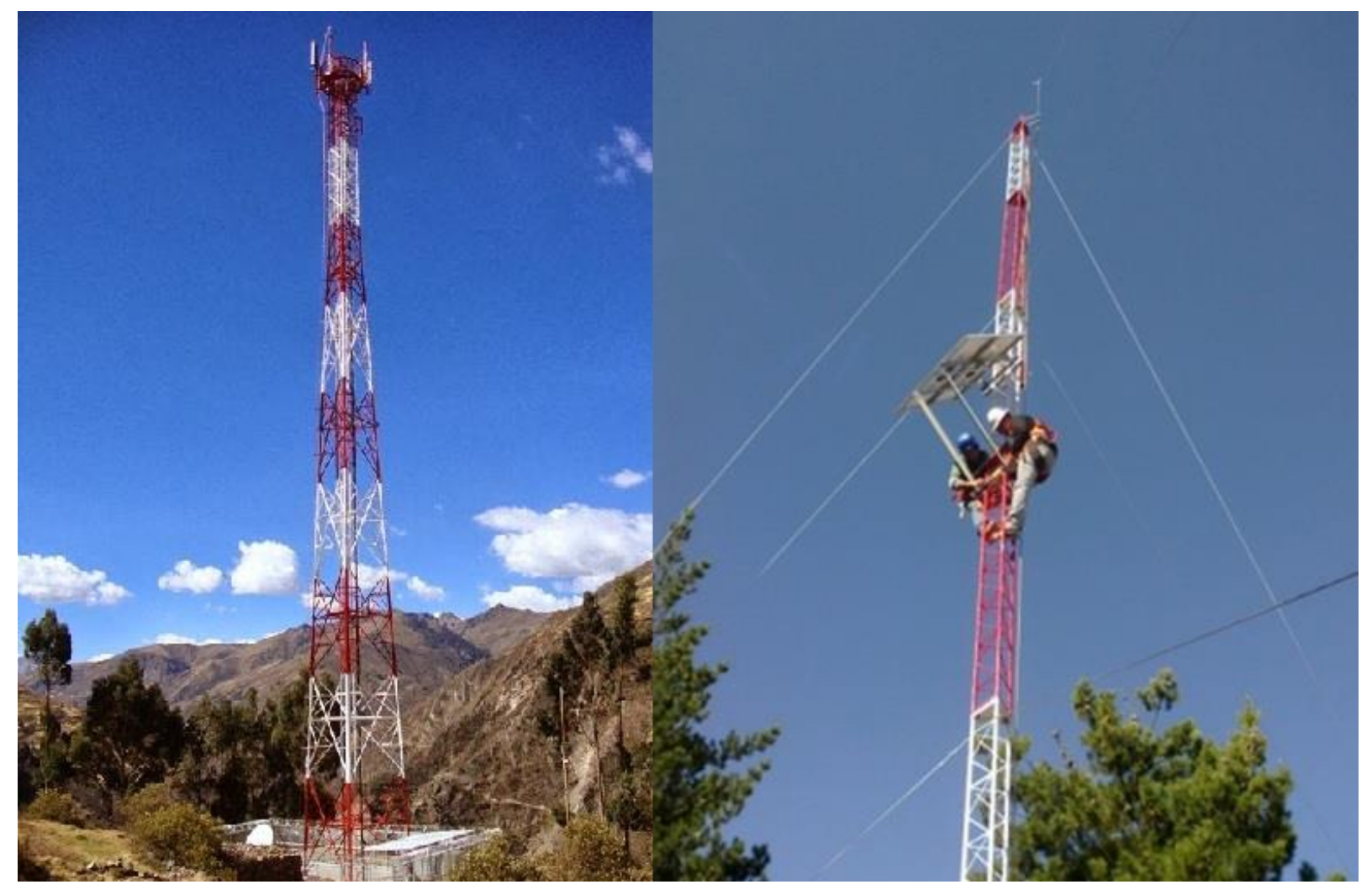

Figure 40. Self-supported tower (Left) and cable supported tower (Right)

Figure 41 shows the total NPV for the WBLANs using self-supported and cable-supported towers in Yauli. The NPV reduces for the case of all technologies, from $4.7 \%$ in the case of Wi-Fi to $15 \%$ in the case of TVWS. Using cable-supported towers, LTE remains the lowest cost option, and TVWS, the highest cost option. 


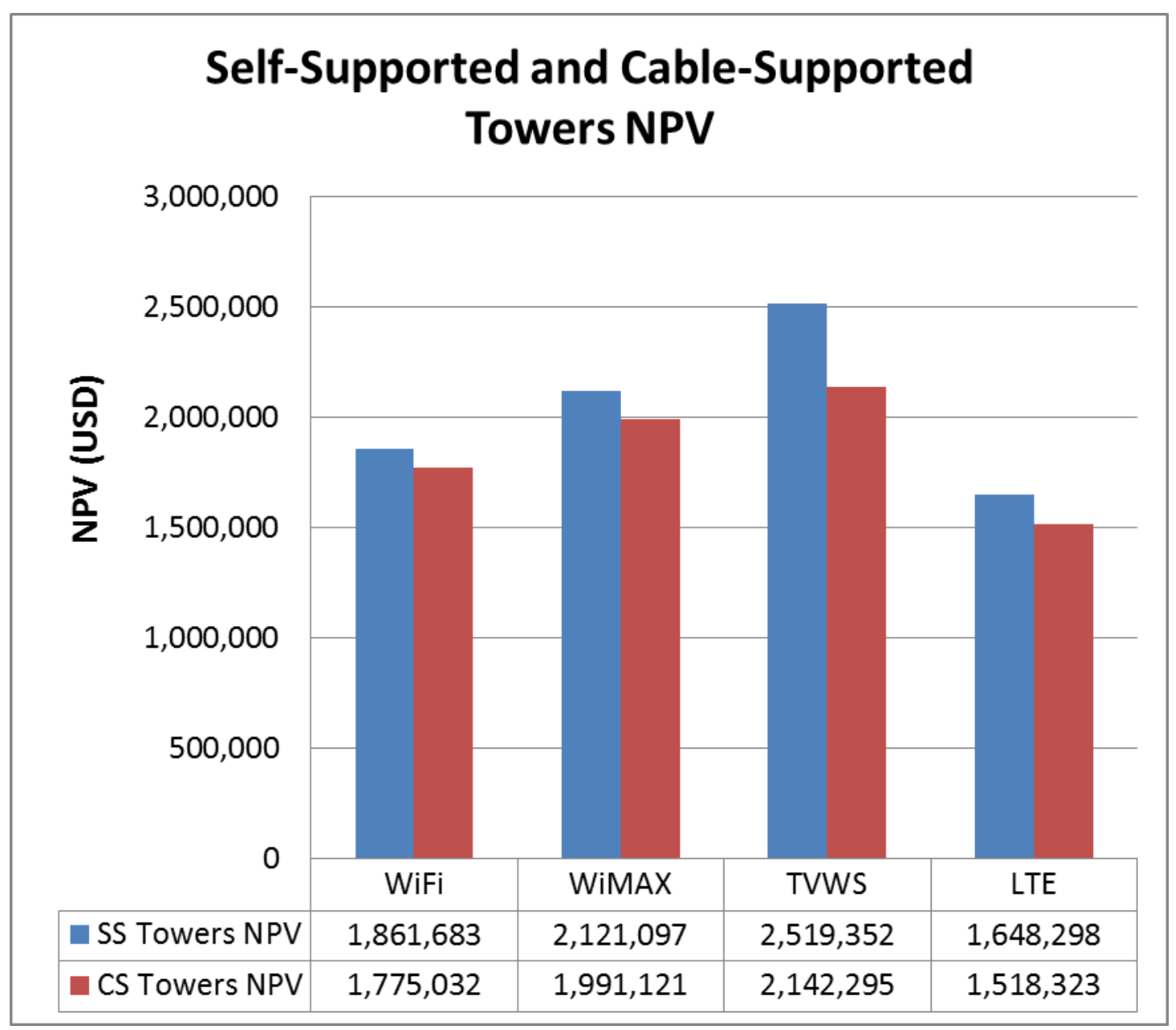

Figure 41. Self-supported and cable-supported towers NPV in Yauli

Figure 42 shows the total NPV for the WBLANs using self-supported and cable-supported towers in Samugari. The NPV reduces for the case of all technologies, from $4.4 \%$ in the case of TVWS to $5.7 \%$ in the case of LTE. Using cable-supported towers, LTE and TVWS remain the lowest and highest cost options, respectively. 


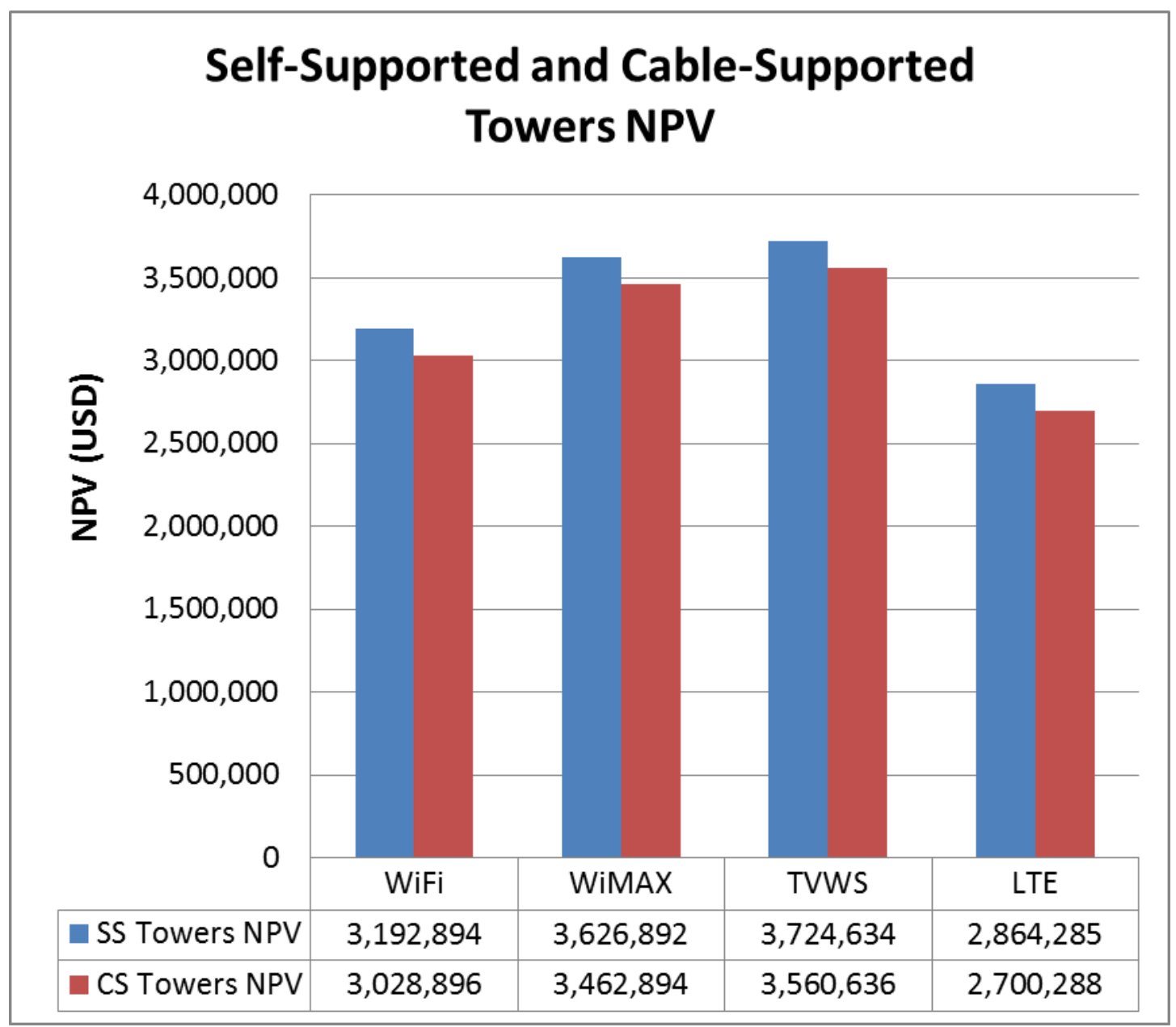

Figure 42. Self-supported and cable-supported towers NPV in Samugari 


\section{CHAPTER V}

\section{REGULATORY AND POLICY IMPLICATION \&}

\section{BROADBAND TECHNOLOGY ROADMAP}

\subsection{Regulatory and Policy Implications}

\subsubsection{Spectrum Proposed Spectrum Scenario: 1.7/2.1GHz LTE band using 40+40MHz spectrum}

In the 2012 work document Caps for the Radioelectric Spectrum Assignment for Public Mobile Services [76], the Peruvian Ministry of Transportation and Communications (MTC) covered the following main points: 1) analysis of spectrum caps application for mobile services in Peru, 2) evaluation of currently assigned spectrum, 3) review of international spectrum trends, and 4) generation of policy recommendations to be implemented in the Communications Sector. This document was based on a spectrum concentration analysis which included different scenarios to achieve efficient spectrum use, preventing spectrum hoarding, and promoting competition and market development. The document concludes (regarding spectrum caps) the following: 
- The $60 \mathrm{MHz}$ spectrum cap per operator should apply only to the $800 \mathrm{MHz}$, $900 \mathrm{MHz}$ and $1900 \mathrm{MHz}$ bands.

- The spectrum cap for the $1.7 / 2.1 \mathrm{GHz}$ band should be set at $40 \mathrm{MHz}$ $(20+20 \mathrm{MHz})$ per operator. This band used to be included among the bands with the total $60 \mathrm{MHz}$ spectrum cap.

- Future spectrum assignment for mobile services must continue monitoring international development and advancement on spectrum regulation in order to improve specific cap policies.

In August 2012, these recommendations became part of the Supreme Decree 011-2012-MTC which updated spectrum caps to be used for public mobile services [80]. In 2013, using the updated spectrum caps, the Peruvian government auctioned two nationwide 1.7/2.1GHz LTE licenses to Movistar and Americatel for USD 152.23 and 105.51 Million [104]. Each license was for a $20+20 \mathrm{MHz}$ block of the 1.7/2.1GHz band. In the last two years, these operators started rolling out LTE services in profitable cellular markets [105] [106] [107] [108], mostly located in main urban areas, and is expected that the LTE market will continue growing in these areas. However, technical and market conditions are different in rural markets. Telecom service providers in rural areas will have to operate under difficult circumstances, including: 1) high-cost conditions due to a rough, undeveloped landscape, 2) predominately low income households, and 3) large geographic regions with a very low density of customers. Results of this analysis indicate that imposing the $1.7 / 2.1 \mathrm{GHz}$ spectrum cap in rural areas may serve as a 
major entry barrier for operators aiming to use LTE to provide both mobile and fixed service rather than promoting a competitive market for the benefit of customers.

This subsection presents the WBLAN design results for the scenario of using 1.7/2.1GHz LTE with $40+40 \mathrm{MHz}$ spectrum; which is double of the current spectrum cap in this band set per operator of $20+20 \mathrm{MHz}$. The amount of spectrum assigned to each technology and caps set per operator have an impact on the effective download channel capacity and capacity (Mbps and users) per node, and as a result, have also significant impact on the WBLAN design and cost.

\section{WBLAN Design for 1.7/2.1GHz LTE using 40+40MHz spectrum}

Table 12 shows the effective download channel capacity and download capacity per node for a WBLAN in the Yauli area using LTE $40+40 \mathrm{MHz}$ (called LTE $40 \mathrm{MHz}$ in this chapter) at different average broadband speed per customer over the 10 -year period. LTE $40 \mathrm{MHz}$ uses two $20 \mathrm{MHz}$ download channels and achieves effective download channel capacity per BS of 107Mbps to 109.7Mbps, which is similar to LTE $20 \mathrm{MHz}$ and higher than Wi-Fi, WiMAX, and TVWS.

Table 13 shows the effective download channel capacity and download capacity per node for LTE $40 \mathrm{MHz}$ in Samugari. In this area, LTE $40 \mathrm{MHz}$ achieves effective download channel capacity of 110.4 to $111.1 \mathrm{Mbps}$, which is similar to LTE $20 \mathrm{MHz}$ and higher than the other technologies. The capacity in this area is slightly higher than in the Yauli area due to the shorter distance between towns and network nodes, so LTE is able to connect to CPEs using higher modulation rates. 
Table 12. Effective download channel and node capacity at different broadband speeds for LTE $40+40 \mathrm{MHz}$ in Yauli

\begin{tabular}{|c|c|c|c|c|c|c|c|}
\hline LTE & \multicolumn{3}{|c|}{ Channel Capacity } & & \multicolumn{3}{|c|}{ Node Capacity } \\
\hline $\begin{array}{c}\text { Avg. BB } \\
\text { Speed Per } \\
\text { Customer }\end{array}$ & $\begin{array}{c}\text { DL Chan } \\
\text { BW } \\
(\mathrm{MHz})\end{array}$ & $\begin{array}{c}\text { Eff. DL Chan } \\
\text { Capacity } \\
\text { (Mbps) }\end{array}$ & $\begin{array}{c}\text { Max. \# } \\
\text { Users Per } \\
\text { Chan (10:1) }\end{array}$ & $\begin{array}{c}\text { \# of DL Chan } \\
\text { (Available } \\
\text { Spectrum) }\end{array}$ & $\begin{array}{c}\text { DL BW } \\
\text { Per } \\
\text { Node } \\
(\mathrm{MHz})\end{array}$ & $\begin{array}{c}\text { Eff. DL } \\
\text { Capacity } \\
\text { (Mbps) }\end{array}$ & $\begin{array}{c}\text { Max. \# } \\
\text { Users } \\
\text { Per } \\
\text { Node } \\
\text { 10:1 }\end{array}$ \\
\hline $2 \mathrm{Mbps}$ & 20 & 107 & 536 & 6 & 40 & 578.0 & 2894 \\
\hline $4 \mathrm{Mbps}$ & 20 & 107 & 268 & 6 & 40 & 578.0 & 1447 \\
\hline $8 \mathrm{Mbps}$ & 20 & 109.7 & 138 & 6 & 40 & 592.2 & 745 \\
\hline $12 \mathrm{Mbps}$ & 20 & 108.5 & 90 & 6 & 40 & 586.1 & 486 \\
\hline
\end{tabular}

Table 13. Effective download channel and node capacity at different broadband speeds for LTE $40+40 \mathrm{MHz}$ in Samugari

\begin{tabular}{c|c|c|c|c|c|c|c|}
\cline { 7 - 8 } \multicolumn{1}{c|}{$\begin{array}{c}\text { LTE } \\
40 \mathrm{MHz}\end{array}$} & \multicolumn{3}{c|}{} & \multicolumn{3}{c|}{ Node Capacity } \\
\hline $\begin{array}{c}\text { Avg. BB } \\
\text { Speed Per } \\
\text { Customer }\end{array}$ & $\begin{array}{c}\text { DL Chan } \\
\text { BW } \\
(\mathrm{MHz})\end{array}$ & $\begin{array}{c}\text { Eff. DL } \\
\text { Chan } \\
\text { Capacity } \\
\text { (Mbps) }\end{array}$ & $\begin{array}{c}\text { Max. \# } \\
\text { Users Per } \\
\text { Chan (10:1) }\end{array}$ & $\begin{array}{c}\text { \# of DL Chan } \\
\text { (Available } \\
\text { Spectrum) }\end{array}$ & $\begin{array}{c}\text { DL BW } \\
\text { Per Node } \\
\text { (MHz) }\end{array}$ & $\begin{array}{c}\text { Eff. DL } \\
\text { Capacity } \\
\text { (Mbps) }\end{array}$ & $\begin{array}{c}\text { Max. \# } \\
\text { Users Per } \\
\text { Node 10:1 }\end{array}$ \\
\hline $2 \mathrm{Mbps}$ & 20 & 110.4 & 552 & 6 & 40 & 596.2 & 2981 \\
\hline $4 \mathrm{Mbps}$ & 20 & 110.4 & 276 & 6 & 40 & 596.2 & 1490 \\
\hline $8 \mathrm{Mbps}$ & 20 & 111.1 & 138 & 6 & 40 & 600.2 & 745 \\
\hline $12 \mathrm{Mbps}$ & 20 & 110.4 & 92 & 6 & 40 & 596.2 & 497 \\
\hline
\end{tabular}

Figure 43 shows the number of users and Mbps capacity per node for Wi-Fi, WiMAX, LTE, TVWS, and LTE 40MHz in Yauli. LTE using 40MHz download spectrum has now the highest capacity per node, 578Mbps to 592Mbps. In the case of LTE $40 \mathrm{MHz}$, it is also used three sectors for each of the two $20 \mathrm{MHz}$ download channels, which assuming an efficiency of $90 \%$ per sector allows a total $5.4 \mathrm{x}$ 
capacity increase in comparison to only one $20 \mathrm{MHz}$ sector. LTE $40 \mathrm{MHz}$ provides the most capacity (users and Mbps) per node than the other technologies, followed by LTE $20 \mathrm{MHz}$, and then the other technologies.

Based on spectrum efficiency metrics, the highest capacity is provided by LTE $40 \mathrm{MHz}$ and LTE $20 \mathrm{MHz}$ with similar users and Mbps per MHz.

Figure 44 shows the number of users and Mbps capacity per node for Wi-Fi, WiMAX, LTE, TVWS, and LTE 40MHz in Samugari. LTE 40MHz also has the highest capacity per node, $596.2 \mathrm{Mbps}$ to $600.2 \mathrm{Mbps}$, in comparison to the other technology options. In this area, LTE $40 \mathrm{MHz}$ also provides the most capacity (users and Mbps) per node than the other technologies, and the most capacity per $\mathrm{MHz}$.

This analysis shows that spectrum is a critical factor for LTE to achieve maximum performance. When using $40 \mathrm{MHz}$ download spectrum instead of $20 \mathrm{MHz}$, LTE provides higher capacity (users and Mbps) per node and per MHz. 


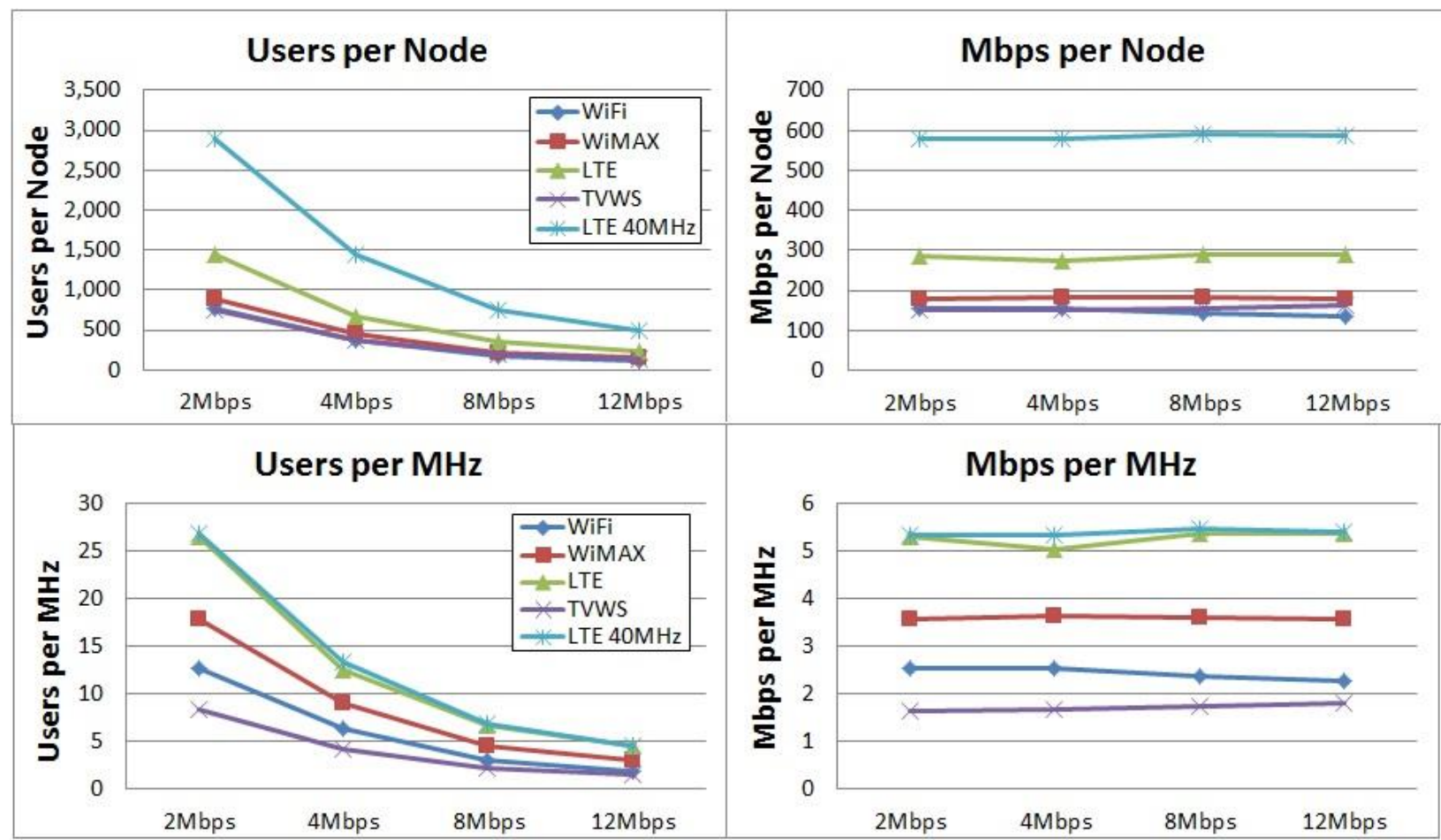

Figure 43. Capacity for wireless technologies in Yauli including LTE 40MHz.

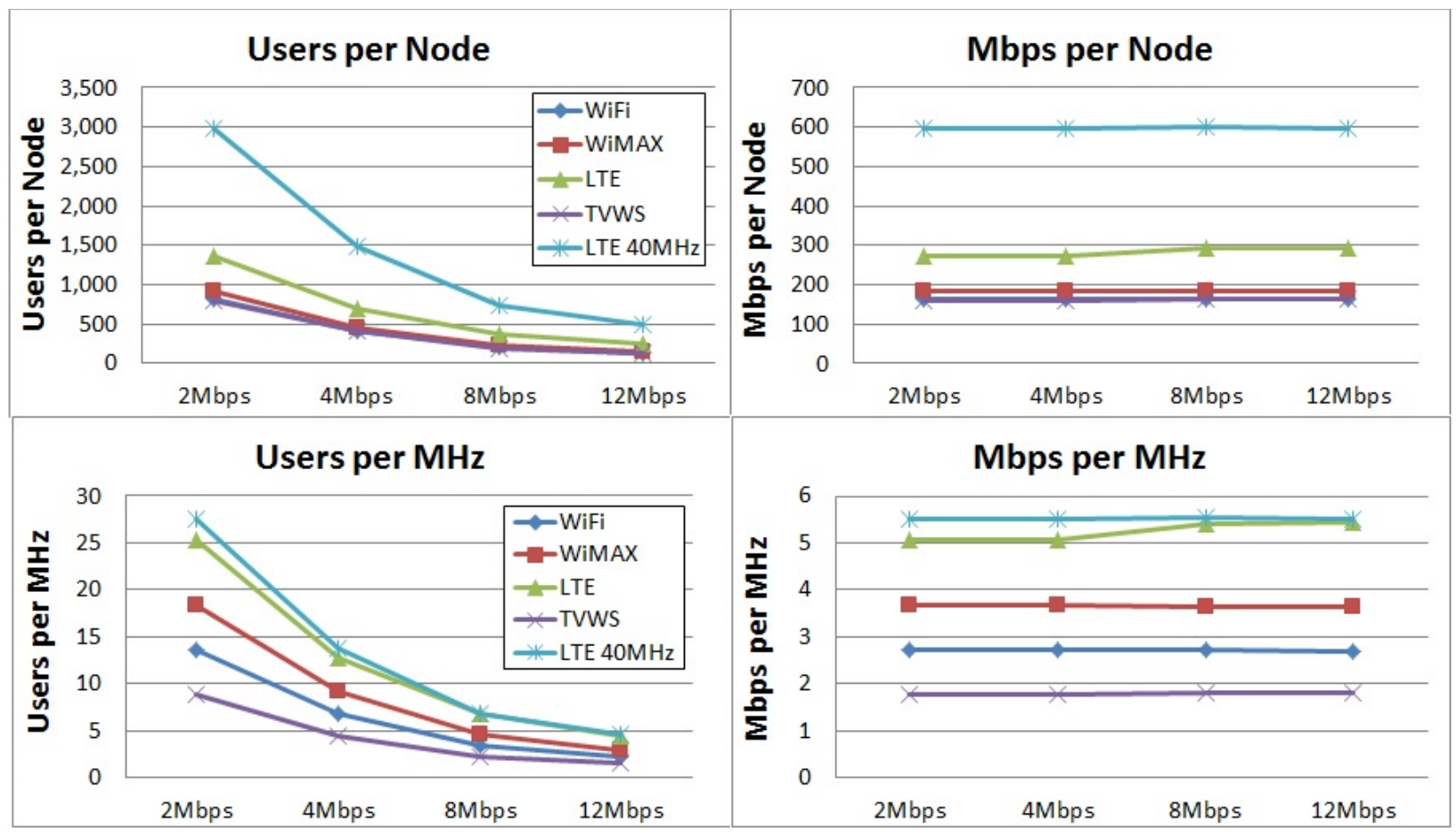

Figure 44. Capacity for wireless technologies in Samugari including LTE 40MHz. 
Table 14 shows the WBLAN design results for LTE $40 \mathrm{MHz}$ for the 10 -year period in Yauli. Comparing the deign results of LTE $40 \mathrm{MHz}$ against the results of Wi-Fi, WiMAX, LTE (20MHz), and TVWS in Yauli (shown in Table 4), in year 1, LTE $40 \mathrm{MHz}$ requires the same number of nodes, 4 , than WiMAX and LTE $20 \mathrm{MHz}$; TVWS requires the least number of nodes, 2 . In years 4,8 and 10, LTE $40 \mathrm{MHz}$ requires equal or the least number of nodes than the other technologies. Regarding the number of base stations, LTE $20 \mathrm{MHz}$ and LTE $40 \mathrm{MHz}$ require least (years 1,4 , 8 and 10) number of base stations than the other technology options. In this area, LTE $20 \mathrm{MHz}$ and LTE $40 \mathrm{MHz}$ require the least number of nodes and BSs due to having the highest effective download channel capacity and capacity per node.

Table 15 shows the WBLAN design results for LTE $40 \mathrm{MHz}$ for the 10 -year period in Samugari. Comparing these results against the ones of Wi-Fi, WiMAX, LTE (20MHz), and TVWS in Samugari (shown in Table 5), in year 1, LTE $40 \mathrm{MHz}$ requires the same number of nodes, 10 , than WiMAX and LTE $20 \mathrm{MHz}$; TVWS requires the least number of nodes, 9 . In year 4 , the number of nodes remains the same for all technologies. In year 8, LTE 20 and LTE $40 \mathrm{MHz}$ require the least number of nodes, 10. In year 10, LTE $40 \mathrm{MHz}$ require the least number of nodes, 10. Regarding the number of base stations, LTE $20 \mathrm{MHz}$ and LTE $40 \mathrm{MHz}$ requires the least number of base stations in all years in comparison to the other technology options due to having the highest effective download channel capacity, which results in a greater client load per base station, and capacity (users and Mbps) per node. 
Table 14. WBLAN design results for the Yauli County for LTE 40MHz.

\begin{tabular}{|r|c|c|c|c|}
\cline { 2 - 5 } \multicolumn{1}{c|}{} & \multicolumn{4}{c|}{ LTE $40+40 \mathrm{MHz}$} \\
\cline { 2 - 5 } & $\begin{array}{c}\text { Y1 } \\
(2 \mathrm{Mbps})\end{array}$ & $\begin{array}{c}\text { Y4 } \\
(4 \mathrm{Mbps})\end{array}$ & $\begin{array}{c}\text { Y8 } \\
(8 \mathrm{Mbps})\end{array}$ & $\begin{array}{c}\text { Y10 } \\
(12 \mathrm{Mbps})\end{array}$ \\
\hline Nodes & 4 & 4 & 4 & 5 \\
\hline Towers & 4 & 4 & 4 & 5 \\
\hline Base Stations/Access Points & 4 & 4 & 12 & 21 \\
\hline Backhaul Radio Links & 3 & 3 & 6 & 11 \\
\hline CPE & 205 & 532 & 1064 & 1368 \\
\hline
\end{tabular}

Table 15. WBLAN design results for the Samugari County for LTE $40 \mathrm{MHz}$.

\begin{tabular}{|r|c|c|c|c|}
\cline { 2 - 5 } \multicolumn{1}{c|}{} & \multicolumn{4}{c|}{ LTE $40+40 \mathrm{MHz}$} \\
\cline { 2 - 5 } & $\begin{array}{c}\text { Y1 } \\
(2 \mathrm{Mbps})\end{array}$ & $\begin{array}{c}\text { Y4 } \\
(4 \mathrm{Mbps})\end{array}$ & $\begin{array}{c}\text { Y8 } \\
(8 \mathrm{Mbps})\end{array}$ & $\begin{array}{c}\text { Y10 } \\
(12 \mathrm{Mbps})\end{array}$ \\
\hline Nodes & 10 & 10 & 10 & 10 \\
\hline Towers & 10 & 10 & 10 & 10 \\
\hline Base Stations/Access Points & 10 & 10 & 17 & 26 \\
\hline Backhaul Radio Links & 9 & 10 & 17 & 27 \\
\hline CPE & 413 & 803 & 1368 & 1675 \\
\hline
\end{tabular}

Engineering cost analysis for $1.7 / 2.1 \mathrm{GHz}$ LTE using $40+40 \mathrm{MHz}$ spectrum

Figure 45 shows the cumulative CAPEX and OPEX of deploying and operating costs of current wireless technology options and LTE $40 \mathrm{MHz}$ in the Yauli area. From year 1 to year 3, TVWS has the lowest cost. Then from year 4 to year 5 , LTE $20 \mathrm{MHz}$ and LTE $40 \mathrm{MHz}$ become the lowest cost option. From year 6 to year 10, LTE $40 \mathrm{MHz}$ becomes the lowest cost option. LTE $40 \mathrm{MHz}$ is followed by LTE $20 \mathrm{MHz}$ from year 6 to 10 . 


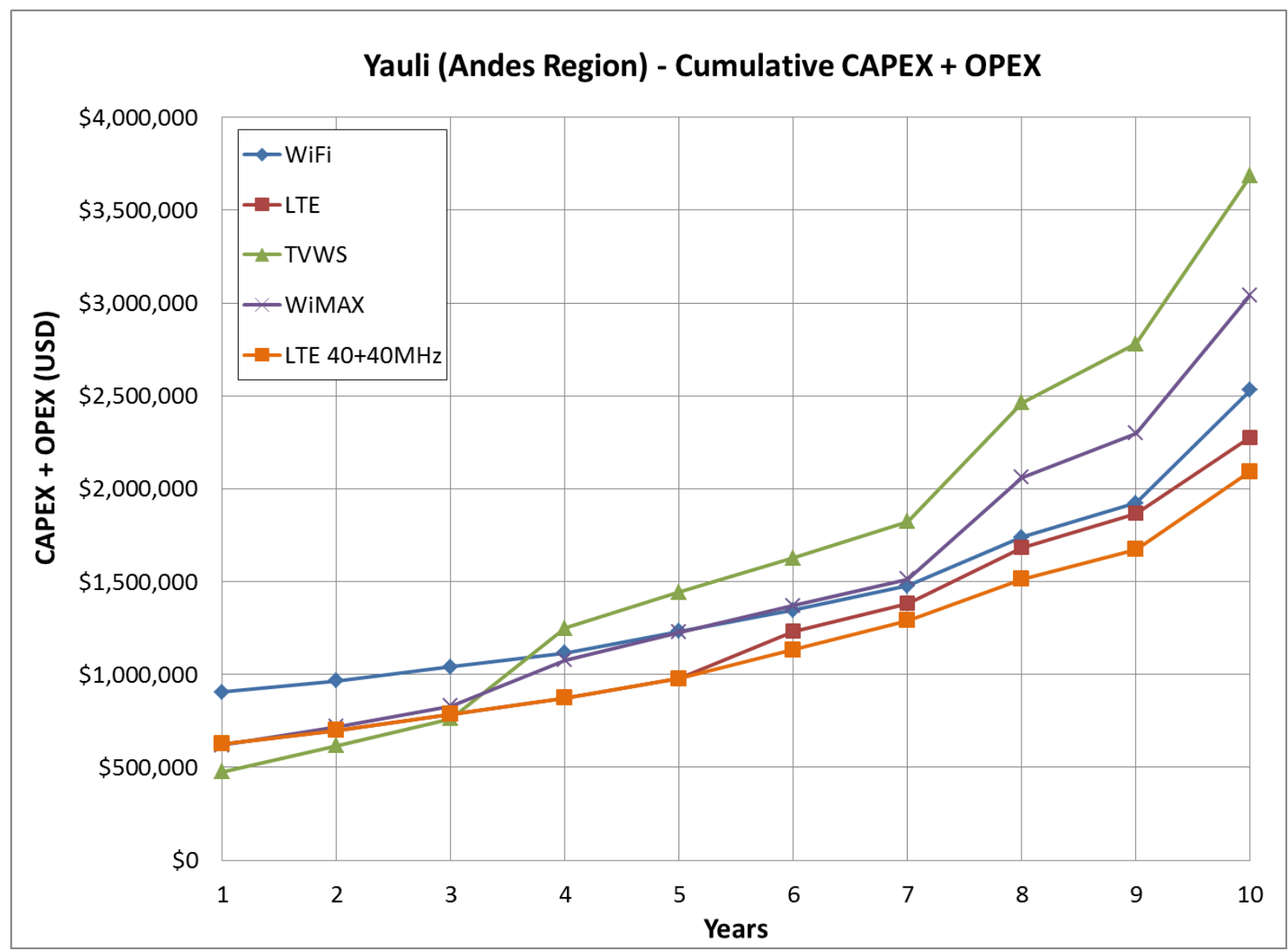

Figure 45. Total cumulative CAPEX and OPEX for a 10-year period for LTE $40 \mathrm{MHz}$ and Wi-Fi, WiMAX, LTE (20MHz) and TVWS in Yauli

Figure 46 shows the cumulative CAPEX and OPEX of deploying and operating costs of current wireless technology options and LTE $40 \mathrm{MHz}$ in the Samugari area. In this area, only in year 1 TVWS has the lowest cost. From year 2 to 9, LTE $20 \mathrm{MHz}$ and LTE $40 \mathrm{MHz}$ has the lowest cost, and year 10, LTE $40 \mathrm{MHz}$ becomes the lowest cost option. 


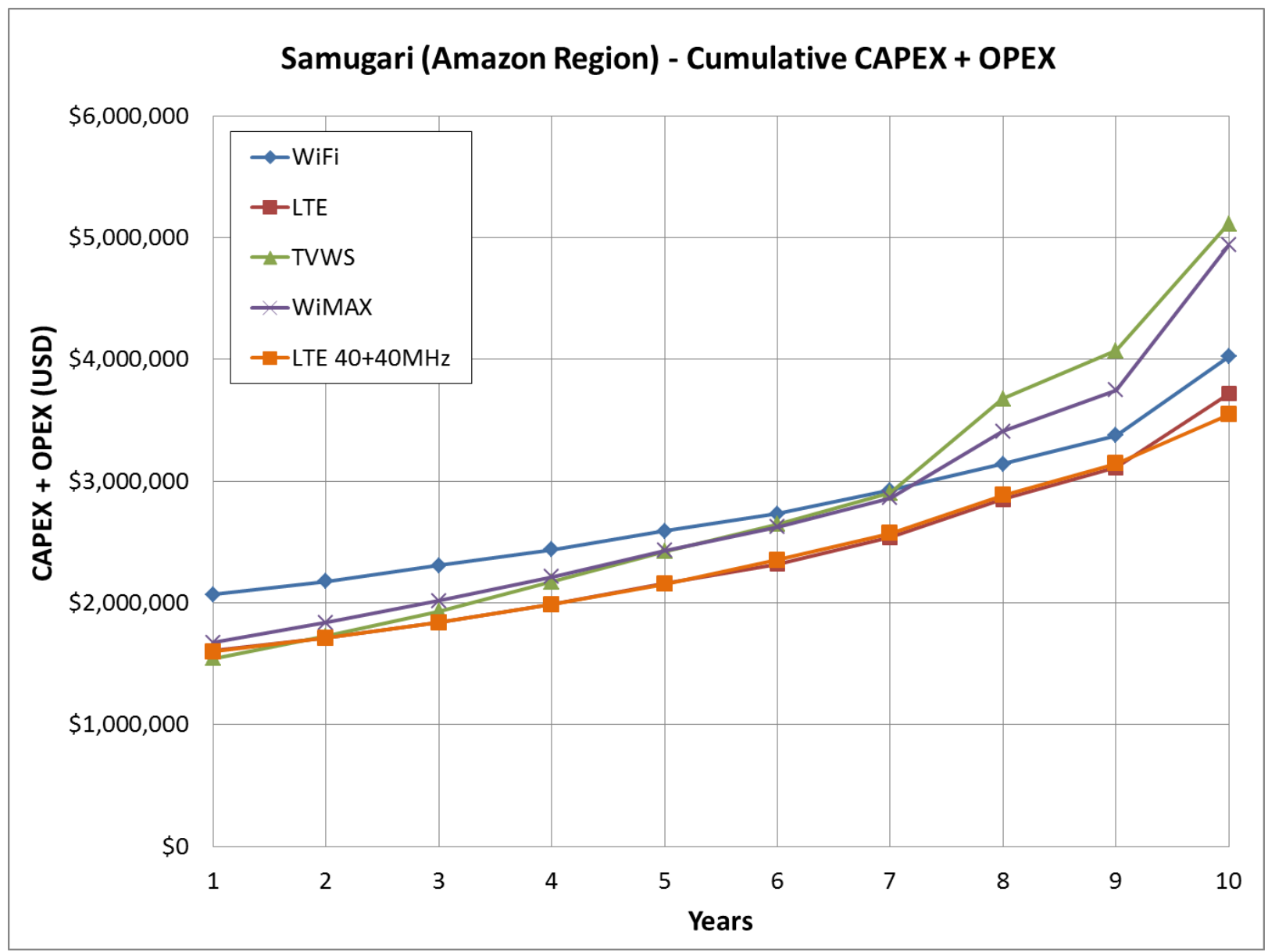

Figure 46. Total cumulative CAPEX and OPEX for a 10-year period for LTE $40 \mathrm{MHz}$ and Wi-Fi, WiMAX, LTE (20MHz) and TVWS in Samugari

Figure 47 shows the NPV of LTE $40 \mathrm{MHz}$ and the current wireless technology options in the Yauli area. LTE $40 \mathrm{MHz}$ comes as the new lowest cost option. LTE (20MHz), Wi-Fi, WiMAX, and TVWS are 7\%, 18\%, 28\% and 39\% more expensive than LTE 40MHz. In the Samugari area, shown in Figure 48, LTE $40 \mathrm{MHz}$ also comes as the new lowest cost option. LTE (20MHz), Wi-Fi, WiMAX and TVWS are $3 \%, 13 \%, 23 \%$ and $25 \%$ more expensive than LTE $40 \mathrm{MHz}$. 


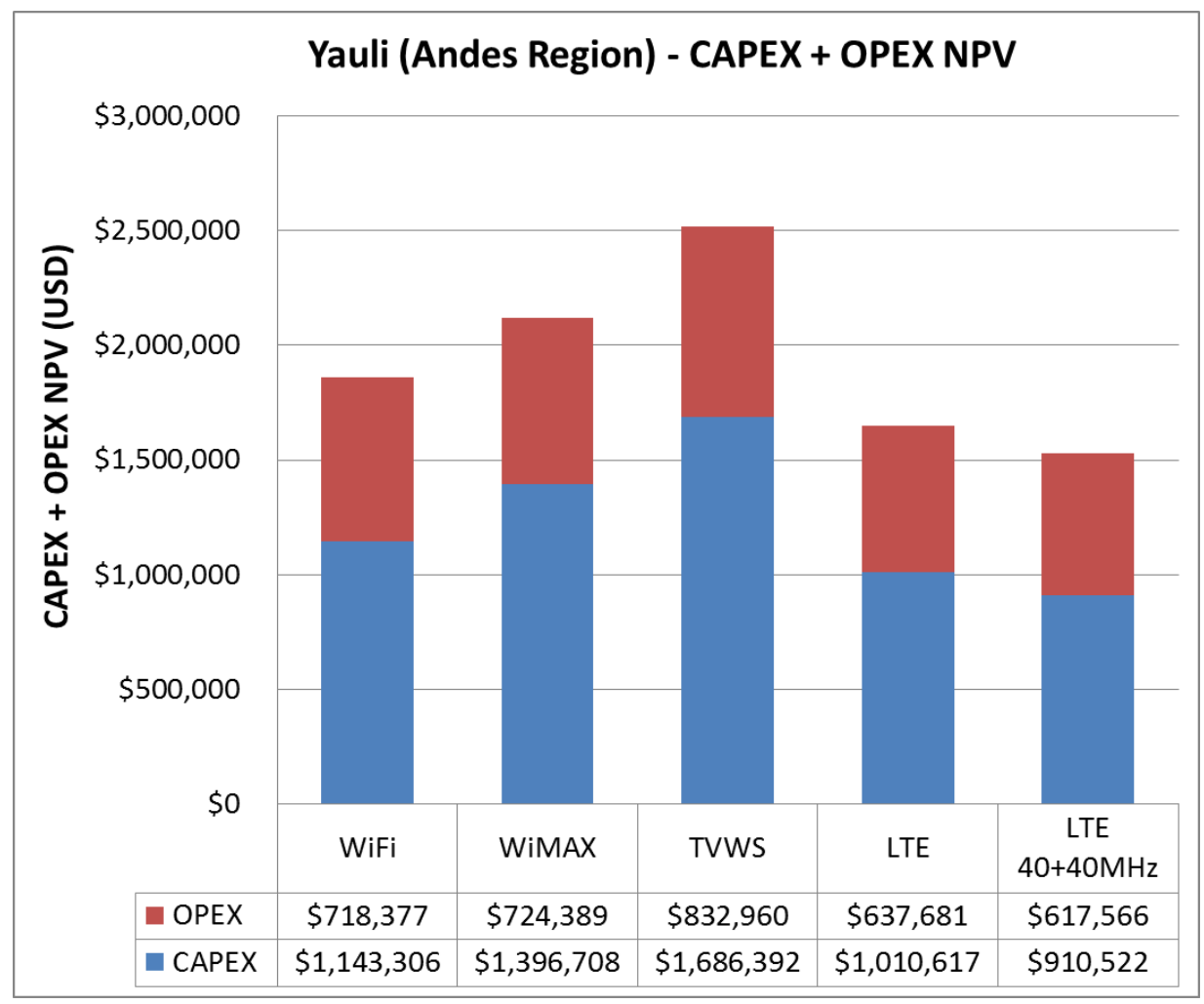

Figure 47. NPV of LTE 40MHz and current wireless options in Yauli

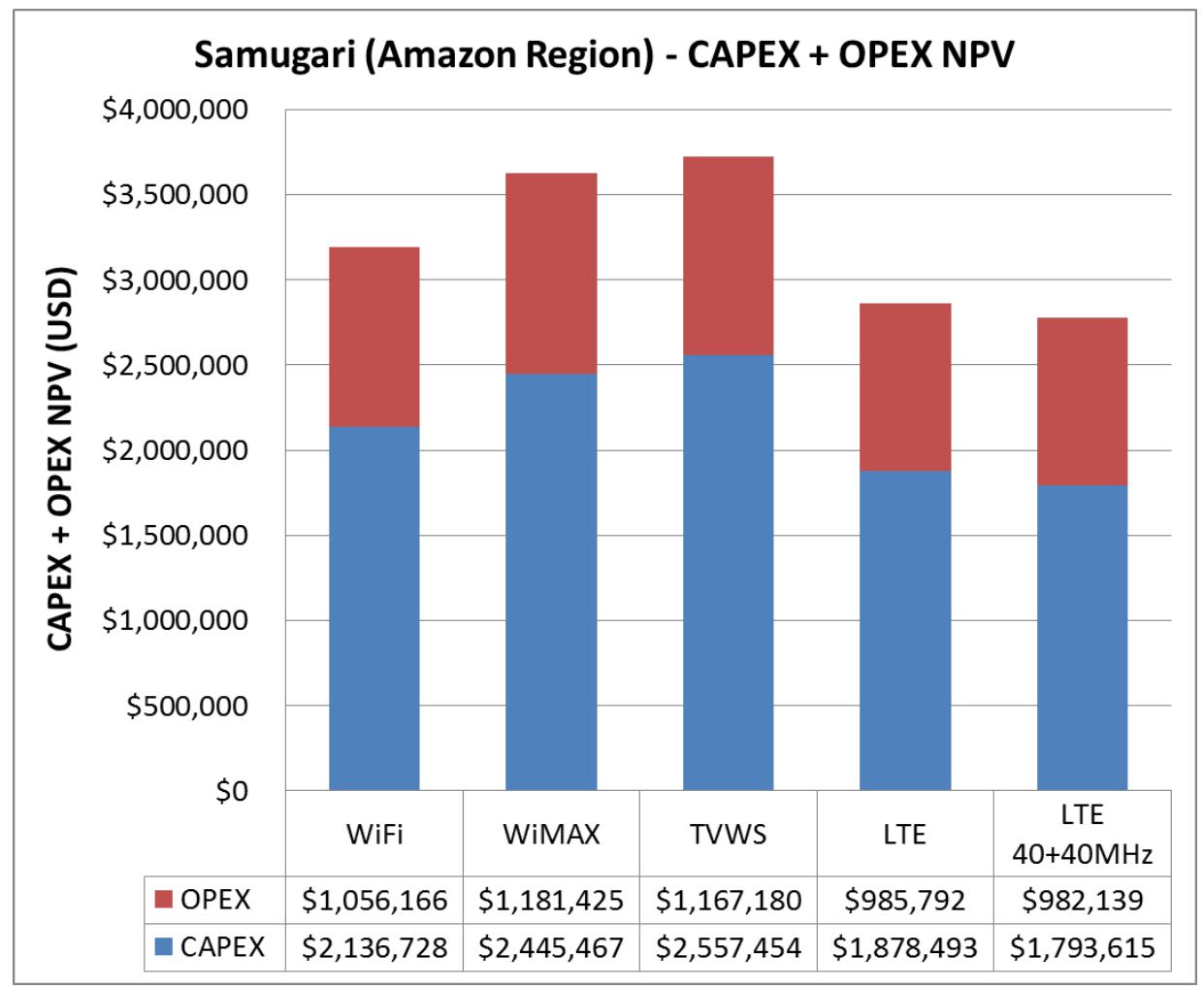

Figure 48. NPV of LTE $40 \mathrm{MHz}$ and current wireless options in Samugari 
Figure 49 and Figure 50 show the cost per user per month for current technologies and LTE 40MHz. In Yauli, in years 1 to 3, TVWS has the lowest cost per user, while LTE $40 \mathrm{MHz}$, LTE $20 \mathrm{MHz}$ and WiMAX have costs close to each other. In years 4 to 5 , LTE $20 \mathrm{MHz}$ and $40 \mathrm{MHz}$ become the lowest cost option, and in years 6 to 10, LTE $40 \mathrm{MHz}$ remain the lowest cost option. Applying the NPV, LTE $40 \mathrm{MHz}$ has now the lowest cost, USD 201. In Samugari, in years 1 to 3 , TVWS, LTE $20 \mathrm{MHz}$ and $40 \mathrm{MHz}$ have the lowest cost per users. In years 4 to 9 , LTE $20 \mathrm{MHz}$ and LTE $40 \mathrm{MHz}$ have the lowest costs, and in year 10, LTE $40 \mathrm{MHz}$ becomes the lowest cost option. Applying the NPV, LTE $40 \mathrm{MHz}$ has now the lowest cost, USD 263. 


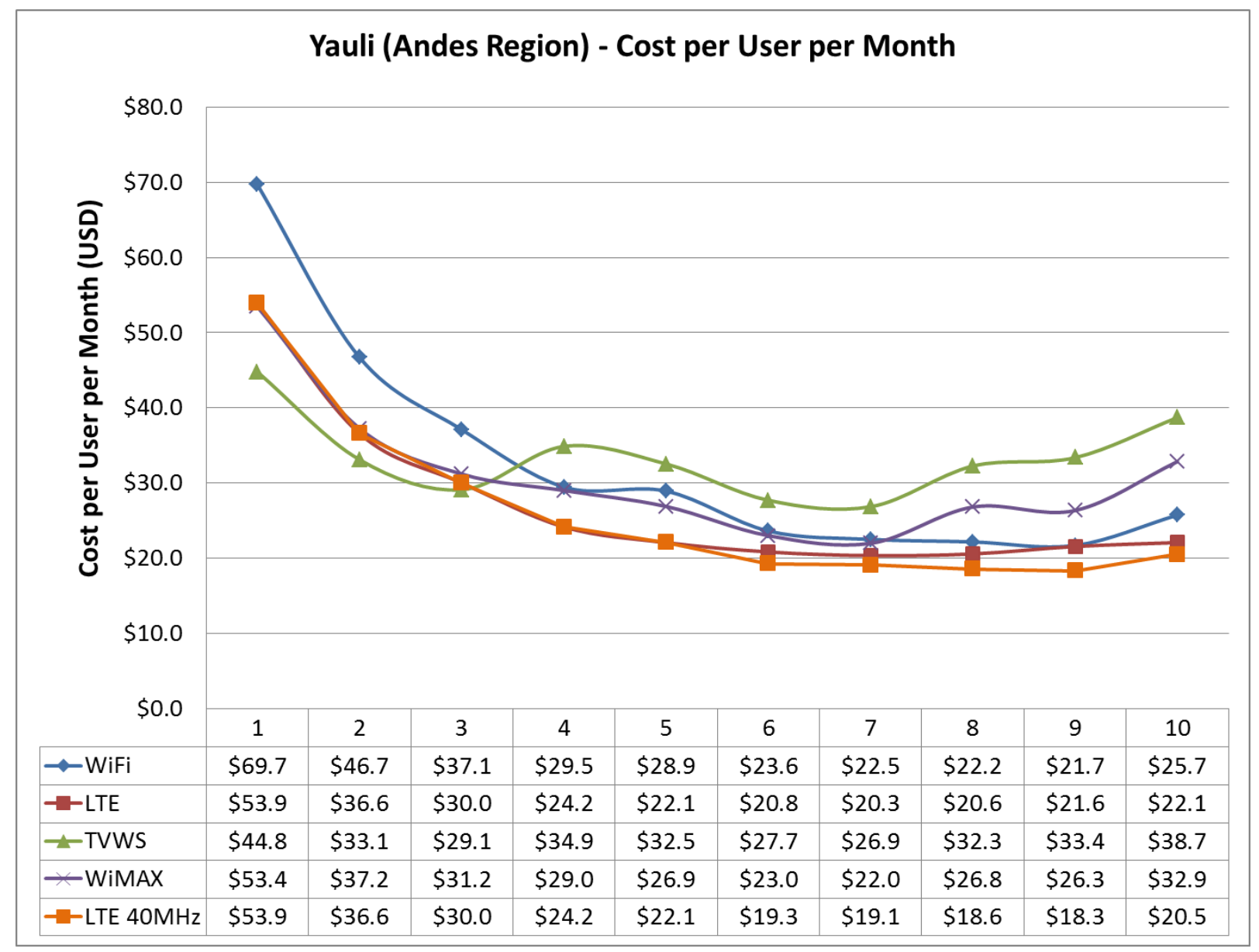

Figure 49. Cost per user per month for the Yauli County including LTE $40+40 \mathrm{MHz}$ 


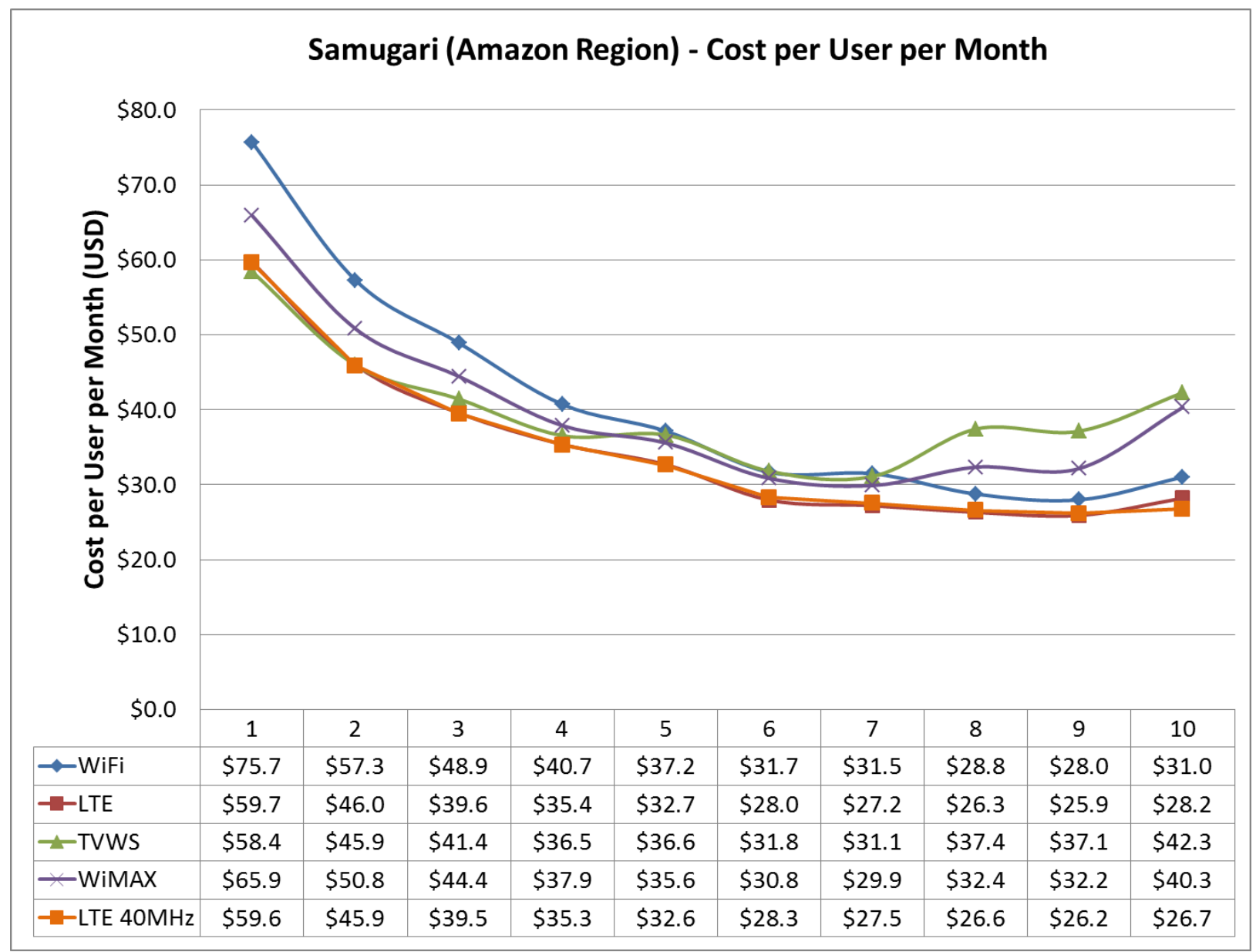

Figure 50. Cost per user per month for the Samugari County including LTE $40+40 \mathrm{MHz}$

Figure 51 and Figure 52 show the cost per Mbps per month for current technologies and LTE 40MHz. In Yauli, in years 1 to 3, TVWS has the lowest cost per Mbps, while LTE 20MHz, LTE 40MHz and WiMAX have costs close to each other. In years 4 to 5 , LTE $20 \mathrm{MHz}$ and $40 \mathrm{MHz}$ become the lowest cost option, and from years 6 to 10, LTE 40MHz becomes the lowest cost option. Applying the NPV, LTE 40MHz has now the lowest cost, USD 73. In Samugari, in year 1, TVWS has the lowest cost per Mbps. In years 2 to 10 , LTE $20 \mathrm{MHz}$ and $40 \mathrm{MHz}$ becomes the lowest cost option. Applying the NPV, LTE $40 \mathrm{MHz}$ has now the lowest cost, USD 91.8 . 


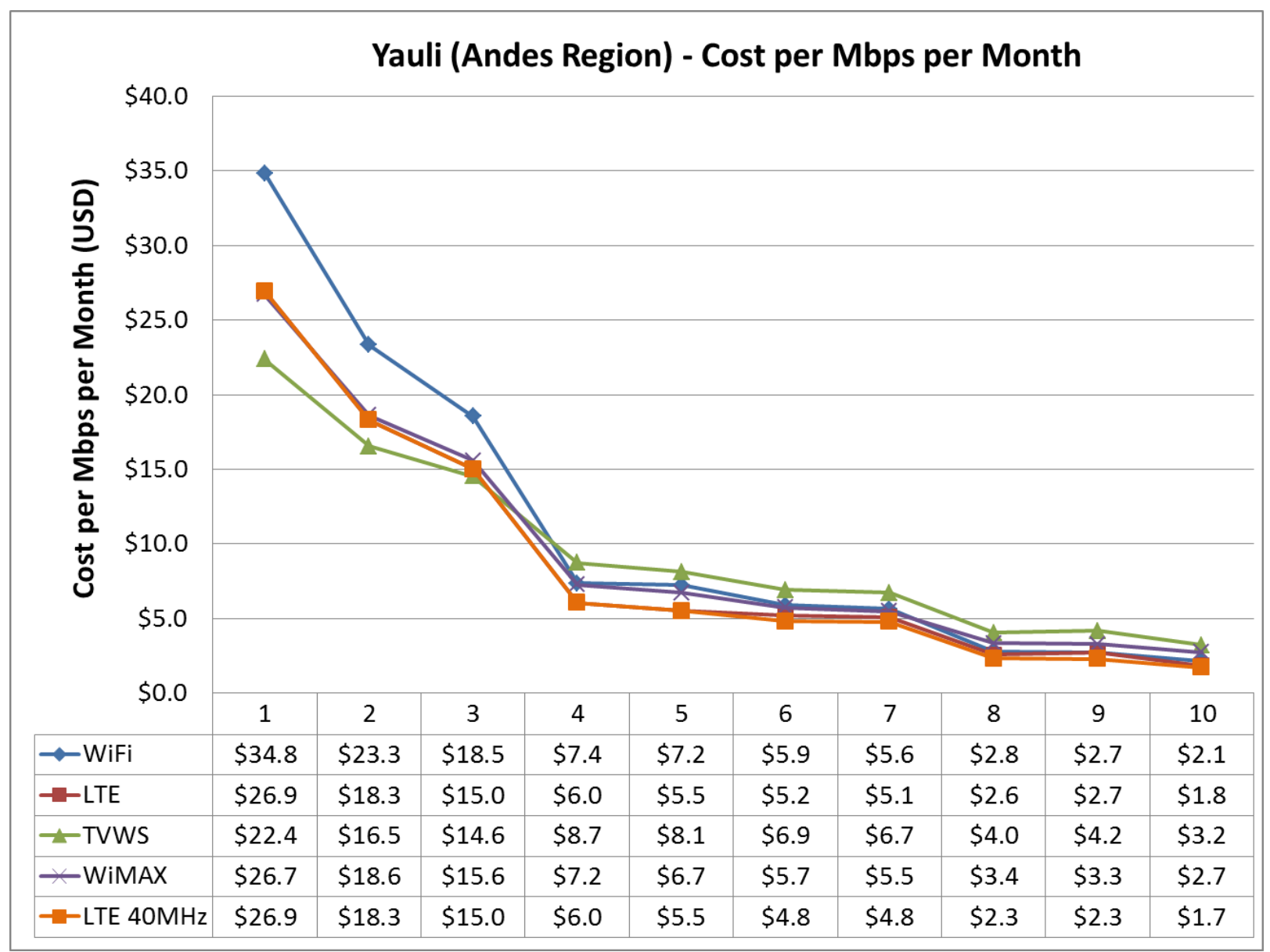

Figure 51. Cost per Mbps per month for the Yauli County including LTE $40+40 \mathrm{MHz}$

Based on the results of the technical and engineering cost analyses, allowing operators in rural areas using $40+40 \mathrm{MHz}$ spectrum (double of current allowed spectrum) in the $1.7 / 2.1 \mathrm{GHz}$ band brings technical and cost efficiencies in the deployment of WBLAN to provide broadband services. LTE $40+40 \mathrm{MHz}$ achieves the highest node capacity in both study areas, leading to require the least number of nodes and BSs. This translates into less expensive infrastructure and network equipment costs. This is shown in the cumulative costs, NPV, cost per user per month and cost per Mbps per month in both study areas. Then this study proposes lifting the $20+20 \mathrm{MHz}$ spectrum cap for the $1.7 / 2.1 \mathrm{GHz}$ LTE band in rural areas in 
order to promote cost efficient deployment and operation of WBLAN to provide broadband services.

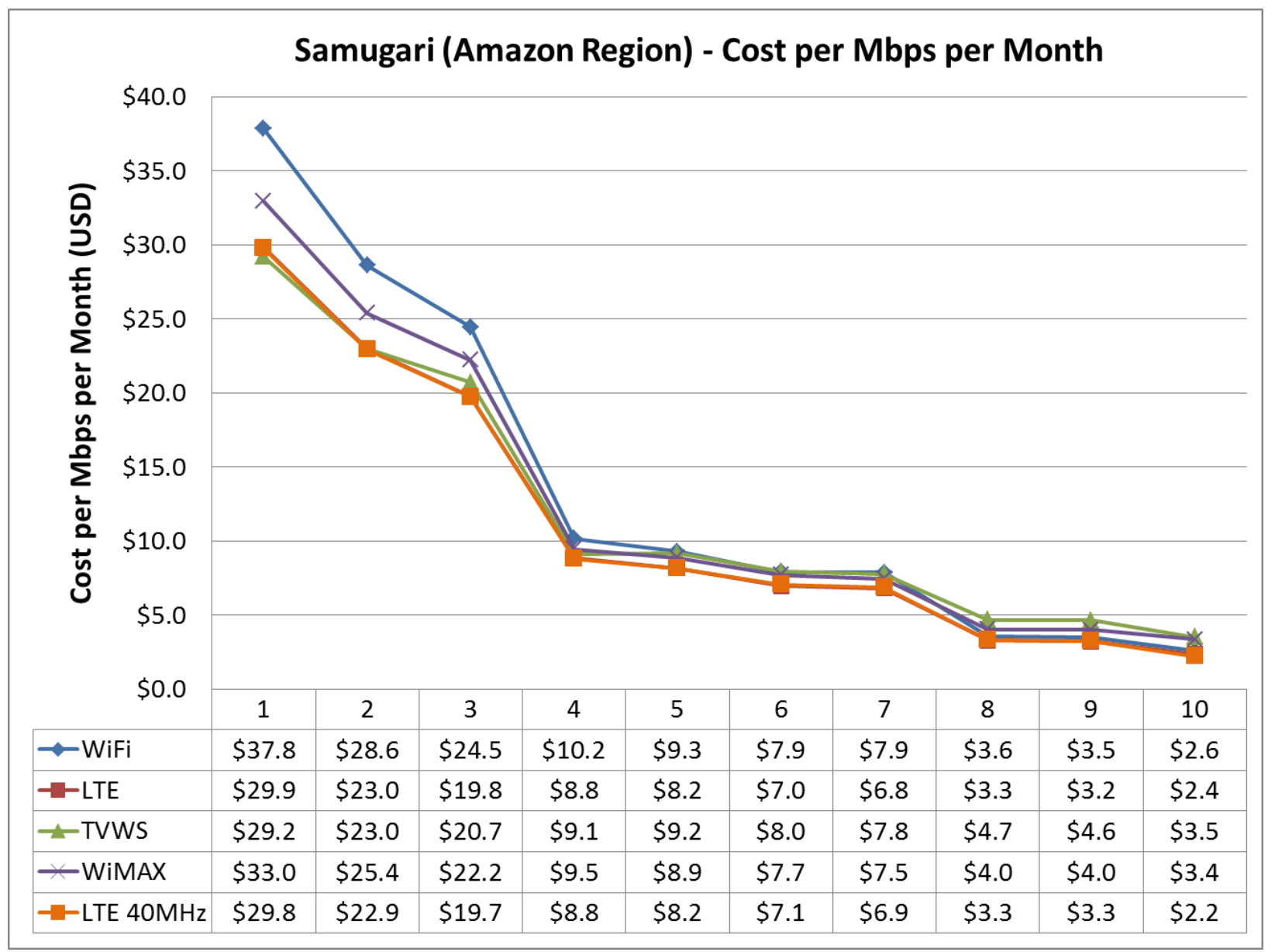

Figure 52. Cost per Mbps per month for the Samugari County including LTE $40+40 \mathrm{MHz}$

\subsubsection{Government subsidy for introducing broadband services in rural areas}

In this subsection, we compare and analyze the amount of government subsidy provided in the FITEL broadband bids for the states of Huancavelica and Ayacucho (where our two study areas are located) against our estimated costs to provide broadband services in the two study areas, Yauli and Samugari, using different wireless technology options. 
Beginning in 2014 and during 2015, the Peruvian government, through FITEL and ProInversion, set public bids for the deployment and operation of broadband networks (fiber optic transport network and wireless local access network) for a 10year period in eight states (each project or bid corresponds to one state). For these bids, broadband service providers competed for a government subsidy to provide fixed broadband services for a 10-year period to government institutions (mandatory or primary customers), and business and residential customers (secondary customers). In March and December 2015, three major broadband service providers were awarded the concession of the broadband projects in these eight states: Gilat (four states), Telefonica (one state) and Redes Andinas (two states).

In this study, we focus on the wireless broadband local access network segment, and compare the deployment and operating costs of using different wireless technology options to provide broadband services in two study areas.

\section{Section 3.1.1 Selection of study areas and access network coverage}

describes the criteria for the selection of the two study areas, the Yauli County, in the Andes Region, and the Samugari County, in the Amazon region; it also covers the towns and customers coverage within the study areas. After selecting the study areas and defining the coverage, we conducted technical and engineering cost analyses of WBLANs using different wireless technologies. These analyses followed and incorporated access network requirements set by FITEL bids regarding to technical specifications (for telecom and energy systems), and infrastructure 
requirements. Some requirements were excluded from the technical and engineering cost analyses due to falling outside of the scope and delimitations of this study (e.g., computer stations, content generation, social awareness campaign, information technology training for customers, NOC deployment, and implementation of a customer service and marketing center).

In the final FITEL bid contracts signed on May 2015 between FITEL and Gilat Networks Peru for the broadband projects for the states of Ayacucho and Huancavelica [85] [86], FITEL granted the subsidy shown in Table 16 for the deployment and operation of transport (optic fiber) and access networks (wireless) in these states for a 10-year period.

Table 16. FITEL's subsidies for the deployment and operation of transport and access networks in the states of Ayacucho and Huancavelica

\begin{tabular}{|l|c|c|c|}
\cline { 2 - 4 } \multicolumn{1}{c|}{} & $\begin{array}{c}\text { Transport Network } \\
\text { Subsidy (USD) }\end{array}$ & $\begin{array}{c}\text { Access Network } \\
\text { Subsidy (USD) }\end{array}$ & $\begin{array}{c}\text { Total FITEL } \\
\text { Subsidy (USD) }\end{array}$ \\
\hline State of Ayacucho & $\$ 37,859,170$ & $\$ 68,555,240$ & $\$ 106,414,410$ \\
\hline State of Huancavelica & $\$ 30,007.148$ & $\$ 67,266,027$ & $\$ 97,273,175$ \\
\hline
\end{tabular}

Since this study focusses only on the access network segment, in this subsection, we compare the subsidy for the access network against our estimated costs using different wireless broadband options. For this purpose, first, the amount of the access network subsidies is adjusted to only the categories included in our technical and engineering cost analyses, and second, the adjusted access network subsidies are compared against our cost estimations. For this comparison 
we use the cost per user per month metric which allows comparing a subsidy for access networks in an entire state against access network cost in only one county.

\section{Adjusted access network subsidy}

The FITEL bids' technical and infrastructure network requirements for the deployments and operation of the access networks are presented in Section 3.2.4 FITEL access network design requirements. For the technical and engineering cost analyses in this study, we included most of these requirements, except for some which fell outside the deployment and operation of access networks from a telecom technical point of view. The excluded items were the following:

- Computer work stations for all government customers (schools, health posts, police stations, etc.).

- Generation of Intranet service and content.

- Social awareness campaign and information technology training for customers.

- Installation and operation of a Network Operating Center for managing all access networks in one state.

- Implementation of a customer service and marketing center.

Based on the feasibility studies made available in the FITEL bid public documents [85] [86], the estimated cost of the excluded items in Ayacucho and Huancavelica are USD 10,804,353 and USD 10,783,917, respectively. The adjusted access network subsidies for both states are shown in Table 17, along with the initial required coverage (towns) for these states. 
Table 17. Adjusted FITEL's subsidy for the access networks and required initial coverage in the states of Ayacucho and Huancavelica

\begin{tabular}{|l|c|c|}
\cline { 2 - 3 } \multicolumn{1}{c|}{} & $\begin{array}{c}\text { Adjusted Access } \\
\text { Network Subsidy (USD) }\end{array}$ & $\begin{array}{c}\text { FITEL Coverage } \\
\text { Towns }\end{array}$ \\
\hline State of Ayacucho & $\$ 57,750,887$ & 350 \\
\hline State of Huancavelica & $\$ 56,482,110$ & 354 \\
\hline
\end{tabular}

Table 18 and Table 19 show the total cost of the WBLANs in Yauli and Samugari using different wireless technologies and also the initial coverage. In our study, to determine the coverage (towns) in the two study areas, we used, first, the FITEL mandatory coverage (FITEL towns and government and residential customers) and, second, also included additional towns which could be served by the WBLANs without significant additional investment (see Section 3.1.1 Selection of study areas and access network coverage). This coverage expansion rationale was based on business practices conducted by telecom operators when deploying infrastructure in rural areas; basically the deployed infrastructure is used to provide as much coverage as possible for the initial network investment.

Table 18. Access network costs in Yauli (Huancavelica) and initial coverage

\begin{tabular}{|l|c|c|c|}
\cline { 2 - 4 } \multicolumn{1}{c|}{} & \multicolumn{1}{c|}{ (USD) } & $\begin{array}{c}\text { FITEL } \\
\text { Coverage } \\
\text { Towns }\end{array}$ & $\begin{array}{c}\text { Additional } \\
\text { Coverage } \\
\text { Towns }\end{array}$ \\
\hline Wi-Fi & $\$ 1,861,683$ & 16 & 11 \\
\hline WiMAX & $\$ 2,121,097$ & 16 & 11 \\
\hline TVWS & $\$ 2,519,352$ & 16 & 11 \\
\hline LTE & $\$ 1,648,298$ & 16 & 11 \\
\hline
\end{tabular}


Table 19. Access network costs in Samugari (Ayacucho) and initial coverage

\begin{tabular}{|l|c|c|c|}
\cline { 2 - 4 } \multicolumn{1}{c|}{} & (USD) & $\begin{array}{c}\text { FITEL } \\
\text { Coverage } \\
\text { Towns }\end{array}$ & $\begin{array}{c}\text { Additional } \\
\text { Coverage } \\
\text { Towns }\end{array}$ \\
\hline Wi-Fi & $\$ 3,192,894$ & 19 & 10 \\
\hline WiMAX & $\$ 3,626,892$ & 19 & 10 \\
\hline TVWS & $\$ 3,724,634$ & 19 & 10 \\
\hline LTE & $\$ 2,864,285$ & 19 & 10 \\
\hline
\end{tabular}

\section{Comparison of FITEL subsidies against calculated WBLAN costs}

For the comparison of the FITEL subsidies of the states of Ayacucho and Huancavelica's broadband bids against the calculated WBLAN costs in the Yauli and Samugari areas, the cost per user per month is used.

For the cost per user per month calculation in both study areas (Yauli and Samugari counties), we used the number of customers estimated in Section 3.1.2 Broadband residential customer and speed demand estimation for a 10year period. This estimation included government and residential customers. For the customer estimation statewide in Huancavelica and Ayacucho, we applied the same residential demand model and also included government customers referred in the bids documents. Once we obtained the customer demand (both government institutions and residential demand), for the states of Huancavelica and Ayacucho and for Yauli and Samugari, the cost per user is calculated. This cost per user per month, in the case of the Ayacucho and Huancavelica states, when multiplied by the annual demand over the 10-year period will amount, using the NPV 
calculation, for the adjusted government subsidy granted for these states. In the case of Yauli and Samugari, it will amount for the NPV of the WBLANs in these study areas.

Table 20 and Table 21 show the cost per user per month in Yauli and Samugari and the subsidy per user per month in the Huancavelica and Ayacucho states. In the case of Samugari and Ayacucho, our cost per user per month is higher than the FITEL subsidy for all technologies. The difference can be observed in the case of using lowest cost LTE equal to USD 7.6 or $22 \%$. This means that based on the estimated customer demand, the subsidy is not able to cover the cost per user. However, the FITEL bids establish that the broadband service provider can charge a monthly rate to all government and residential customers. The rate is not set yet and it will be discussed and approved by the regulator (OSIPTEL). Based on willingness to pay surveys presented in the FITEL bid's feasibility study, our demand model assumed that the Internet monthly service rate will be USD 12.5. Then, charging this rate, the service provider will be able to cover the WBLAN costs.

In the case of Yauli and Huancavelica, our cost per user per month is lower than the FITEL subsidy for all technologies. The greatest difference can be observed in the case of using LTE, USD 16.29 or $58 \%$. This means that the broadband service provider can have a profit during the 10 -year period. Furthermore, in addition to the USD 12.5 Internet service monthly rate, the 
broadband service provider can extend the coverage to more customers in the service area.

In conclusion, based on the cost per user per month, in Ayacucho (Amazon Region scenario), the broadband service provider will be able to break even, and in Huancavelica (Andes region scenario), the broadband service provider will be able to have a profit during the 10-year period.

Table 20. Cost per user per month in Yauli and in Huancavelica

\begin{tabular}{|l|c|c|c|}
\cline { 2 - 4 } \multicolumn{1}{c|}{} & $\begin{array}{c}\text { Cost per user } \\
\text { per month } \\
\text { in Yauli (USD) }\end{array}$ & $\begin{array}{c}\text { Subsidy per user } \\
\text { per month } \\
\text { in Huancavelica (USD) }\end{array}$ & $\begin{array}{c}\% \\
\text { Difference }\end{array}$ \\
\hline Wi-Fi & $\$ 31.6$ & $\$ 44.3$ & $40 \%$ \\
\hline WiMAX & $\$ 36.0$ & $\$ 44.3$ & $23 \%$ \\
\hline TVWS & $\$ 42.8$ & $\$ 44.3$ & $3 \%$ \\
\hline LTE & $\$ 28.0$ & $\$ 44.3$ & $58 \%$ \\
\hline
\end{tabular}

Table 21. Cost per user per month in Samugari and in Ayacucho

\begin{tabular}{|l|c|c|c|}
\cline { 2 - 4 } \multicolumn{1}{c|}{} & $\begin{array}{c}\text { Cost per user } \\
\text { per month } \\
\text { in Samugari (USD) }\end{array}$ & $\begin{array}{c}\text { Subsidy per user } \\
\text { per month } \\
\text { in Ayacucho (USD) }\end{array}$ & $\begin{array}{c}\% \\
\text { Difference }\end{array}$ \\
\hline Wi-Fi & $\$ 38.8$ & $\$ 27.2$ & $-30 \%$ \\
\hline WiMAX & $\$ 44.1$ & $\$ 27.2$ & $-38 \%$ \\
\hline TVWS & $\$ 45.2$ & $\$ 27.2$ & $-40 \%$ \\
\hline LTE & $\$ 34.8$ & $\$ 27.2$ & $-22 \%$ \\
\hline
\end{tabular}




\subsubsection{Overbooking proposed scenario: Maintaining 1:10 oversubscription rate in rural areas}

Until October 2014, among different quality of service regulations, the regulator OSIPTEL set a guaranteed minimum speed of $10 \%$ of the nominal Internet service speed leased by broadband service subscribers. In October 2014, OSIPTEL approved an updated General Regulation for Quality of Public Telecommunication Services [109] in order to improve quality of Internet service provided by broadband service providers and, among several updates on quality of service parameters, it increased the minimum speed from $10 \%$ to $40 \%$ of the nominal Internet service speed, raising concerns from broadband service providers due to additional infrastructure and capacity investments as a result of meeting the required service. Using a more known quality of service parameter, this minimum speed can translate into the overbooking or oversubscription ratio which is a critical parameter used by broadband service provider to plan and estimate the required capacity and equipment (and as a result investment) to serve customer and speed demands. The following formula shows the relationship between guaranteed minimum speed and overbooking ratio:

$$
\text { Overbooking Ratio }=\text { Concurrence Factor } x \text { Minimum Speed }
$$

In urban areas, using a concurrence factor of $50 \%$ and a minimum speed of $10 \%$, the overbooking ratio is $20: 1$. However, using the increased $40 \%$ minimum speed, the overbooking ratio is 5:1. 
In rural areas, using a concurrence factor of $25 \%$ and a minimum speed of $40 \%$, the overbooking ratio is $10: 1$. In the case that over the coming years, the concurrence factor would increase at levels similar to urban areas (40\%-50\%), the overbooking ratio can become close to 5:1.

In this subsection, we present the engineering cost analysis results of using overbooking ratios of 5:1 and 20:1 in LTE deployments (in terrestrial and stratospheric networks), and compare them against the cost results used in our previous analysis using an overbooking ratio of 10:1.

Figure 53 shows the NPV over the 10-year period using LTE in the Yauli area and using different overbooking ratios. In this area, using the overbooking ratio of 5:1 results in a 49\% NPV increase in comparison to using 10:1. On the other hand, using a 20:1 results in an 18\% NPV reduction. 


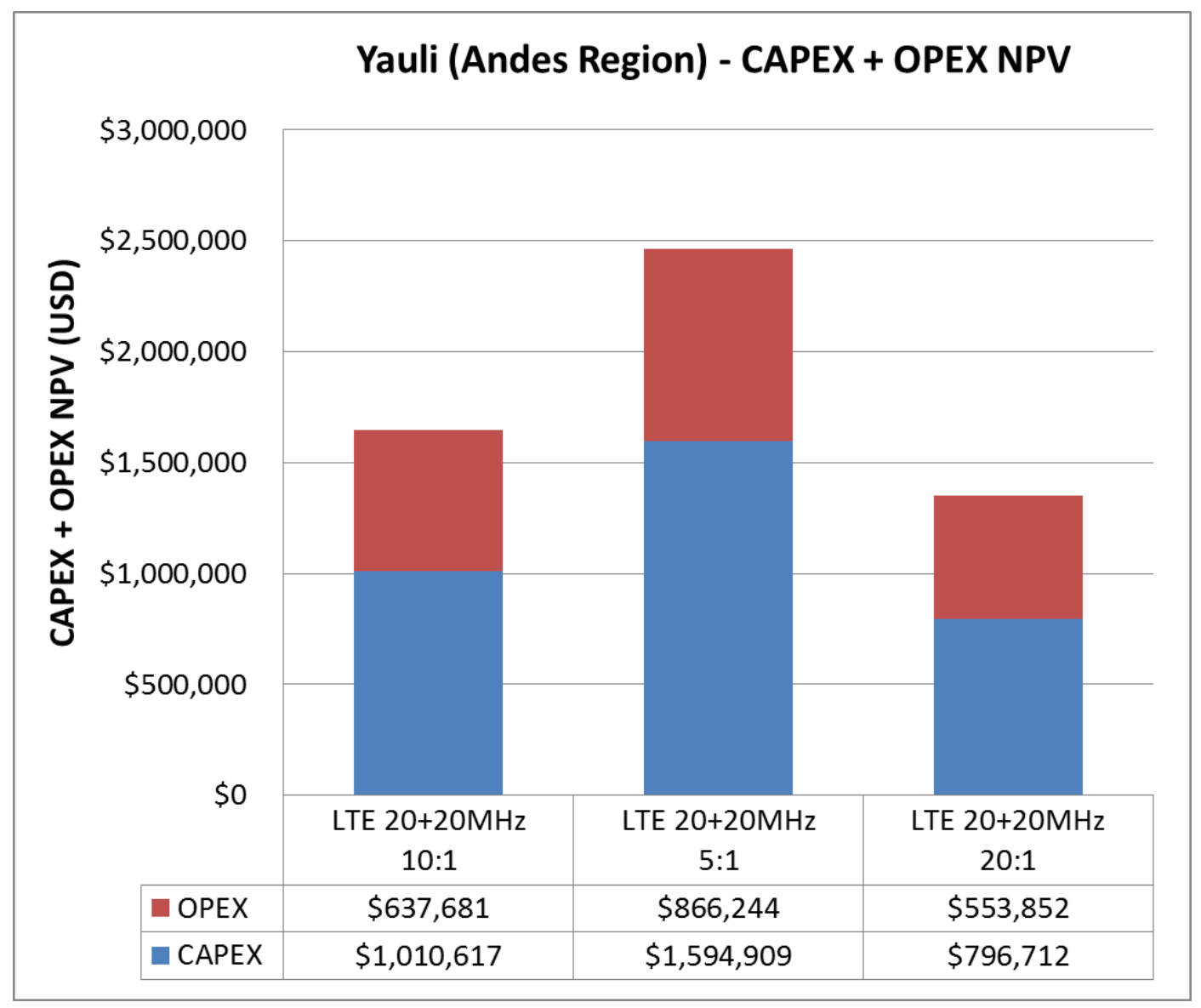

Figure 53. NPV of LTE 20+20MHz in Yauli at different oversubscription rates: 10:1, 5:1, and 20:1.

Figure 54 shows the NPV over the 10-year period using LTE in the Samugari area and using different overbooking ratios. In this area, using the overbooking ratio of 5:1 results in a 40\% NPV increase in comparison to using 10:1. On the other hand, using a 20:1 results in an $13 \%$ NPV reduction. 


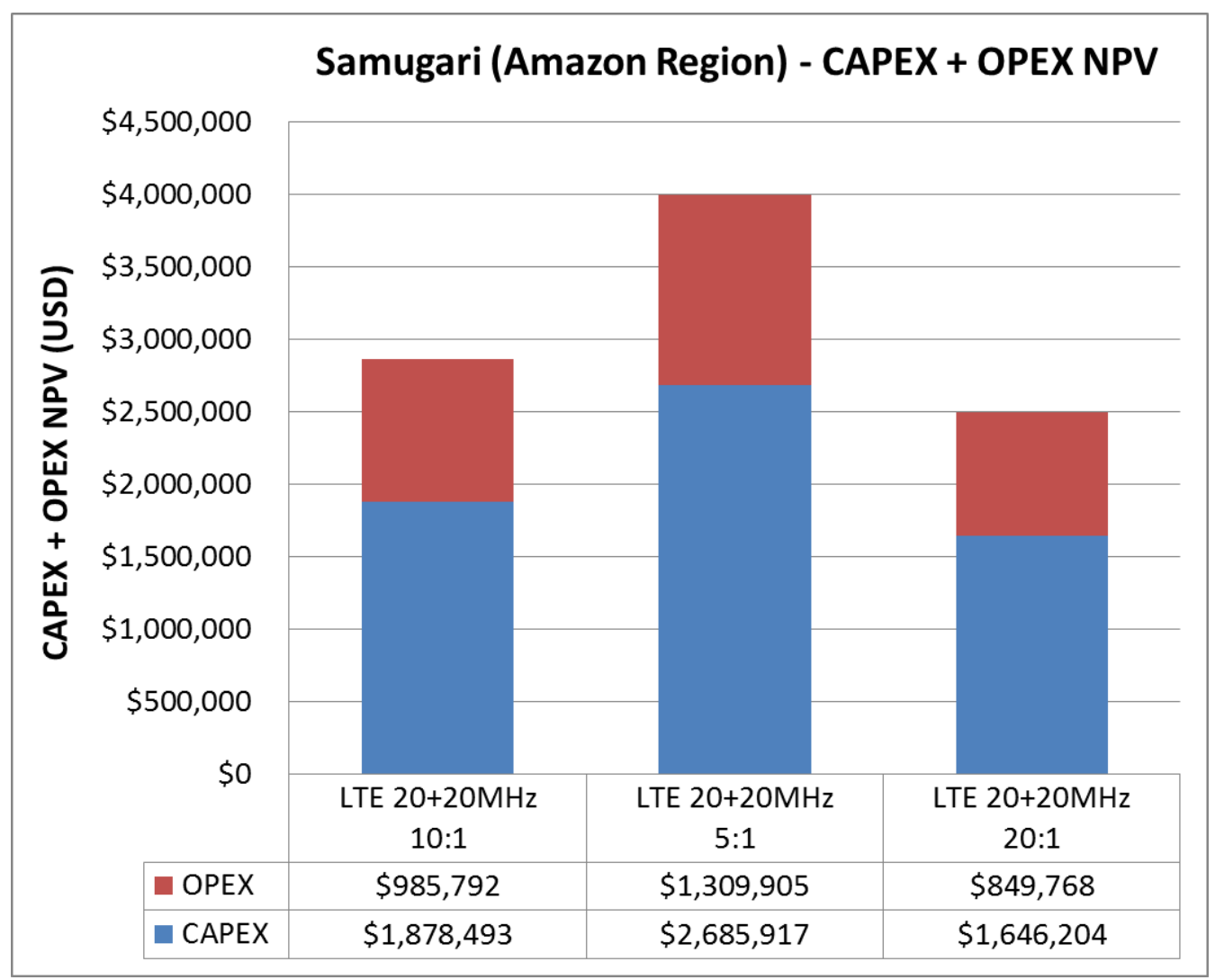

Figure 54. NPV of LTE 20+20MHz in Samugari at different oversubscription rates: 10:1, $5: 1$, and 20:1.

Figure 55 shows the cumulative CAPEX and OPEX over the 10-year period using stratospheric balloons carrying micro-LTE BSs in the Yauli area and using different overbooking ratios. As mentioned in the previous chapter, in this area, using an overbooking ratio of 10:1 allows stratospheric balloons to serve the customer and speed demands for five years. When using an overbooking of $5 ; 1$, balloons can handle the demand only for three years in addition to experience an increase on cumulative costs in these three years. On the other hand, using a overbooking 20:1 allows balloons to serve this area for seven years in addition to show a slightly cumulative cost reduction in the first five years in comparison to 
the overbooking case of 10:1. It's important to note that in neither cases balloons can handle the customer and speed demands over the entire 10-year period.

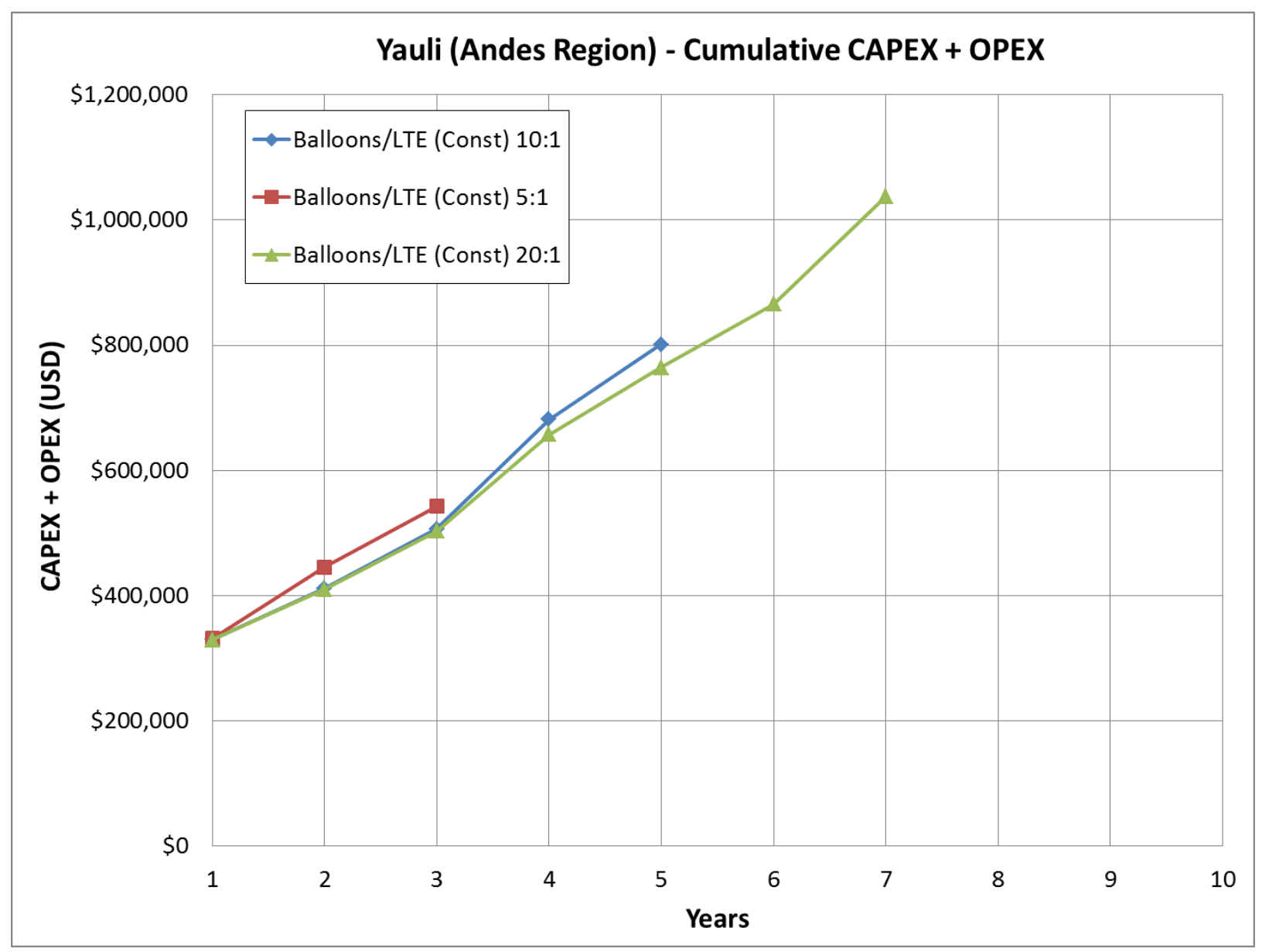

Figure 55. Total cumulative CAPEX and OPEX for the deployment and operation of stratospheric balloons in the Yauli area using overbooking ratios of 10:1, 5:1 and 20:1.

Figure 56 shows the cumulative CAPEX and OPEX over the 10-year period using stratospheric balloons carrying micro-LTE BSs in the Samugari area and using different overbooking ratios. As mentioned in the previous chapter, in this area, using an overbooking ratio of 10:1 allows stratospheric balloons to serve the customer and speed demands for seven years. When using an overbooking of $5 ; 1$, balloons can handle the demand only for three years in addition to experience an 
increase on cumulative costs in these three years. On the other hand, using a overbooking 20:1 allows balloons to serve this area for eight years in addition to show a slightly cumulative cost reduction in the first seven years in comparison to the overbooking case of 10:1. It's important to note that in neither cases balloons can handle the customer and speed demands over the entire 10-year period.

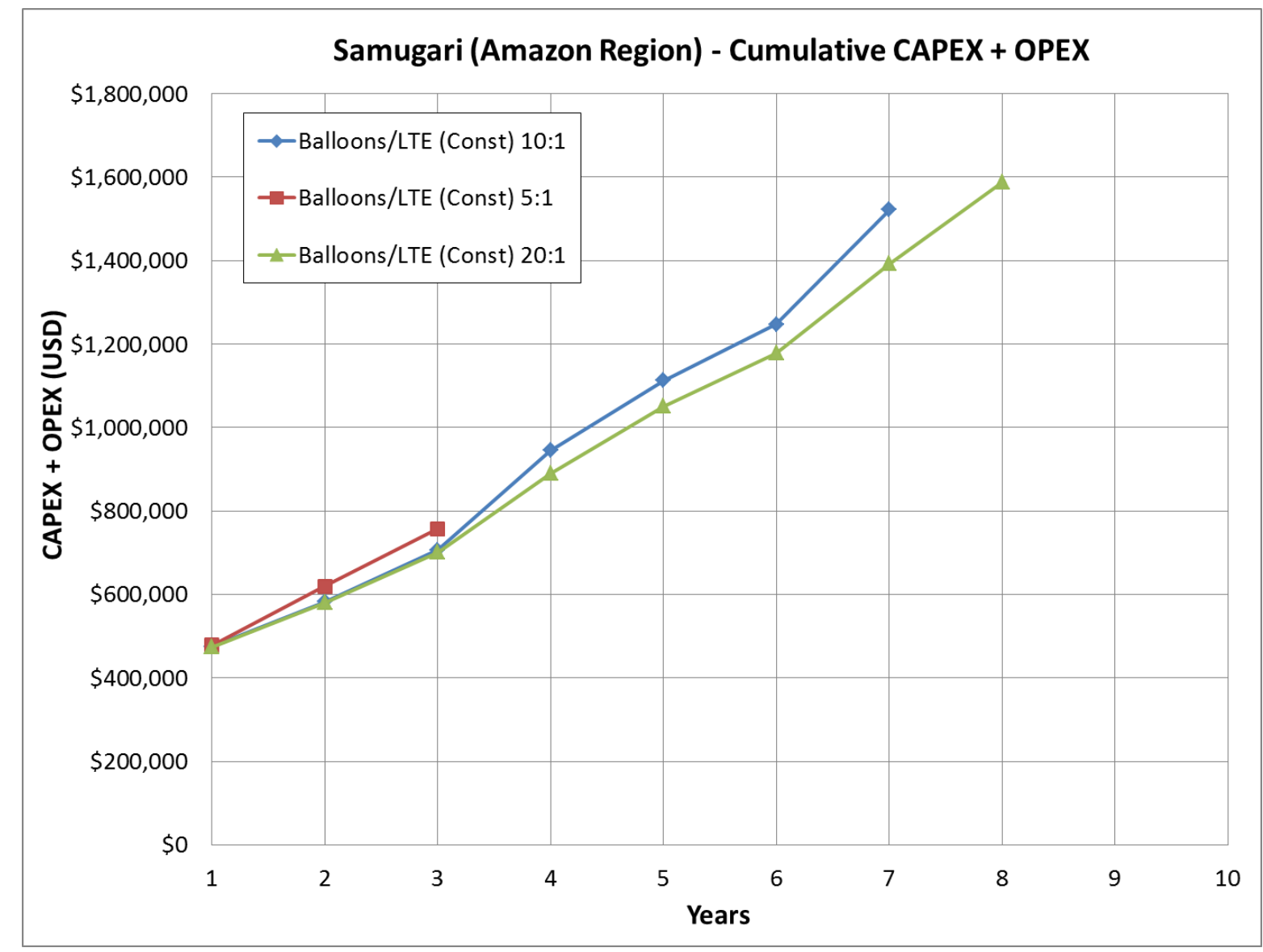

Figure 56. Total cumulative CAPEX and OPEX for the deployment and operation of stratospheric balloons in the Samugari area using overbooking ratios of 10:1, 5:1 and 20:1.

In conclusion, based on the technical and engineering cost analyses, allowing operators in rural areas to use an overbooking ratio of 10:1 in addition to the subsidy provided by the universal agency FITEL would generate incentives for 
operators to compete for these subsidies and carry out access network deployments. In the case that OSIPTEL's quality of service mandates would require operators to use an overbooking ratio of 5:1, operators' required investment would increase by almost $50 \%$ and $40 \%$ in the Andes and Amazon regions, respectively. In these conditions, this overbooking factor would become an infrastructure deployment barrier. On the other hand, allowing an overbooking ratio of 20:1 at least for the first years of deployment, while the broadband demand base is just taking off, would lead to an $18 \%$ and $13 \%$ infrastructure deployment investment reduction, increasing the incentive of broadband operators to carry out deployments in rural areas. Then we recommend to maintain the overbooking ratio of 10:1 and in some cases, especially in high cost and low demand areas, allowing deployments under an overbooking ratio of 20:1. 


\subsection{Broadband technology roadmap for a 10-year period}

This subsection presents our proposed broadband technology roadmap to provide broadband services to unserved and underserved rural areas in the Peruvian Andes and Amazon regions for a 10-year period. To develop this roadmap, first, in the previous chapters, we analyzed and compared technical and cost aspects of using current and new wireless technology options and stratospheric platforms for deploying and operating WBLANs, and second, in this section, we combine the best technical features and cost efficiencies of the analyzed technology options into an innovative roadmap solution for a 10-year period.

Chapter 4 presents the technical and engineering cost analyses results in both study areas of using current wireless technology options (Wi-Fi $2.4 \mathrm{GHz}$, WiMAX 3.5GHz, TVWS 476-786MHz and LTE 1.7/2.1GHz) and also stratospheric balloons using LTE 1.7/2.1GHz. Section 4.1.2 Stratospheric Access Networks shows in the technical analysis that, in the Yauli and Samugari areas, stratospheric balloons would be able to serve the broadband and speed demands for the five and seven years, respectively (see Figure 25 and Figure 26). Section 4.2.1

Current Technologies shows in the engineering cost analysis that, in both study areas, stratospheric balloons (both constellation and single balloon configurations) have the lowest initial costs (see Figure 27 and Figure 28). Section 4.2.1 also shows that, in both study areas, among the terrestrial technologies which can serve customer and speed demands for the 10-year period, LTE was the lowest cost 
option based on the net present value of the deployment and operation costs for the 10-year period (see Figure 29). In this chapter, Section 5.1 Regulatory and Policy Implications presented the technical and engineering cost results of using 1.7/2.1 LTE with a $40+40 \mathrm{MHz}$ spectrum, based on a scenario of lifting the $20+20 \mathrm{MHz}$ spectrum cap per operator of the $1.7 / 2.1 \mathrm{GHz}$ band. The results showed that LTE $40+40 \mathrm{MHz}$ becomes the lowest cost option in both study areas (see Figure 47 and Figure 48).

Then, to combine the best technical features and cost efficiencies of the analyzed technology options to develop our optimal technology roadmap for a 10year period, we start by analyzing scenarios which include the following:

1 Using stratospheric balloons (both constellation and single balloon configurations) for the deployment and operation of the WBLAN for the first years due to their lower deployment and operation costs during these years in comparison to the terrestrial options. Additionally, LTE in the balloon payload includes scenarios of using $20+20 \mathrm{MHz}$ and $40+40 \mathrm{MHz}$ spectrum.

2 When balloons reach its maximum capacity, the WBLAN will be complemented with deployment and operation of the lowest cost terrestrial WBLAN options: LTE $20+20 \mathrm{MHz}$ and LTE $40+4 \mathrm{MHz}$ (in comparison to current technology options and in the scenario of lifting the LTE spectrum cap).

The following subsections present the technical and engineering cost analyses of the scenarios mentioned above. 


\subsubsection{Technical Analysis}

The first years of the roadmap uses stratospheric balloons carrying a 1.7/2.1GHz micro-LTE base station. In the Yauli and Samugari areas, balloons (one balloon in Yauli and two balloons in Samugari) using LTE and $20+20 \mathrm{MHz}$ (called in the rest of this section $20 \mathrm{MHz}$ ) spectrum can serve customer and speed demands for 5 and 7 years, respectively, and using $40+40 \mathrm{MHz}$ (called in the rest of this section $40 \mathrm{MHz}$ ) spectrum can serve 7 years in Yauli and 8 years in Samugari (shown in Figure 57).

After the stratospheric balloons carrying micro-LTE base stations (using either $20 \mathrm{MHz}$ or $40 \mathrm{MHz}$ spectrum) reach its maximum capacity to serve customer and speed demands, complementary terrestrial networks using LTE $20 \mathrm{MHz}$ or LTE $40 \mathrm{MHz}$ are deployed. The following subsection presents the engineering cost analysis results of the solutions using stratospheric balloons for the initial deployment and operation years of the WBLANs, and then deploying complementary terrestrial networks using LTE $20 \mathrm{MHz}$ or LTE $40 \mathrm{MHz}$. 


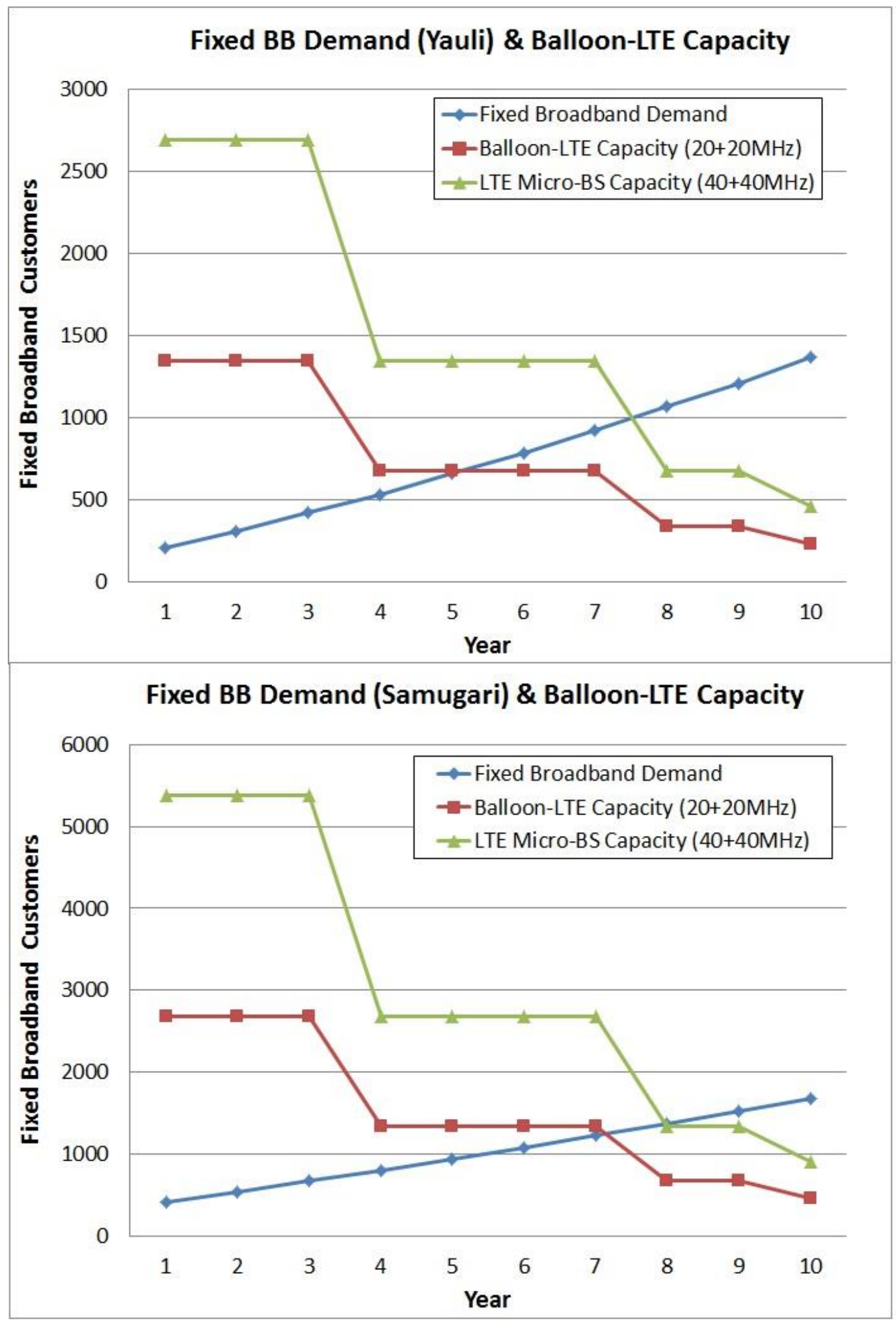

Figure 57. Fixed broadband demand and micro-LTE BS capacity for $20+20 \mathrm{MHz}$ and $40+40 \mathrm{MHz}$ spectrum in Yauli (one balloon) and Samugari (two balloons) 


\subsubsection{Engineering Costs Analysis}

From the analyzed scenarios combining stratospheric balloons (both constellation or single balloon configurations) with complementary terrestrial networks using LTE $20 \mathrm{MHz}$ or $40 \mathrm{MHz}$ spectrum, this subsection presents the engineering cost analysis results of the options which provide costs lower than LTE $20 \mathrm{MHz}$ (lowest cost among current options). Based on current Peruvian spectrum regulations, LTE $20 \mathrm{MHz}$ is the lowest cost technology option for the 10-year period, combined (Yauli and Samugari) NPV of USD 4,512,583. Then proposed candidates for the technology roadmap should provide more cost efficiencies than current options (status quo). The following are the potential technology options for the roadmap and corresponding combined NPV of both study areas (Yauli and Samugari) for the 10-year period (also shown in Figure 58):

- Constellation balloon configuration (LTE $20 \mathrm{MHz}$ ) complemented with LTE $20 \mathrm{MHz}$ (NPV USD 4,330,179)

- Constellation balloon configuration (LTE $40 \mathrm{MHz}$ ) complemented with LTE $20 \mathrm{MHz}$ (NPV USD 4,318,678)

- Terrestrial LTE 40MHz (NPV USD 4,303,841)

- Constellation balloon (LTE 40MHz) complemented with LTE $40 \mathrm{MHz}$ (USD $4,119,963)$

- Constellation balloon (LTE 20MHz) complemented with LTE 40MHz (USD $4,112,415)$ 


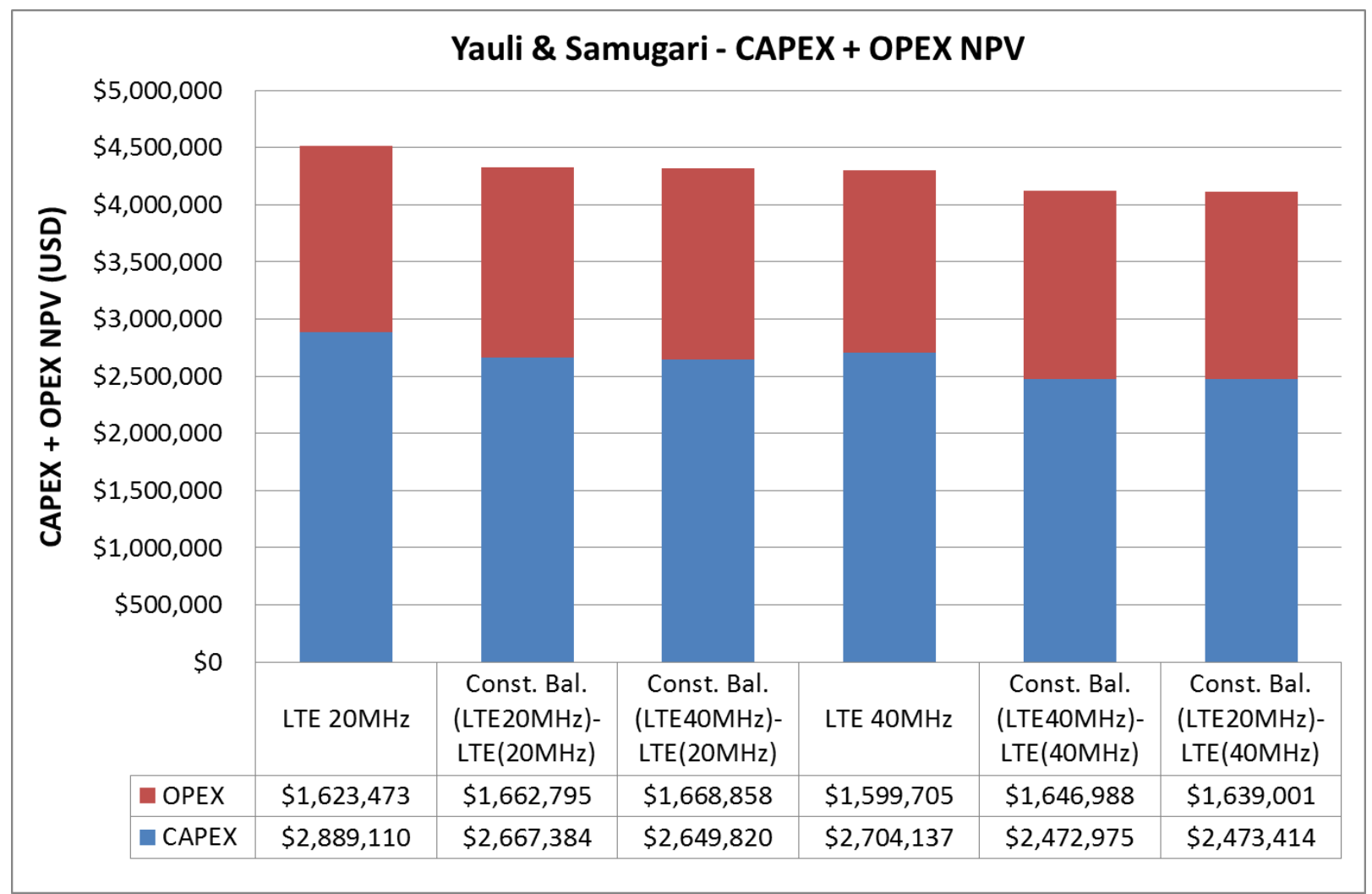

Figure 58. Combined (Yauli and Samugari) NPV of potential roadmap technology options

Figure 59 shows the cumulative CAPEX and OPEX of the combined (both Yauli and Samugari areas) deployment and operation costs of the potential roadmap technology options. This figure helps to understand the cost dynamics and efficiencies of using stratospheric balloons in the first years and then using complementary LTE networks until year 10 .

In years 1 to 5 (2Mbps and $4 \mathrm{Mbps}$ average broadband speed demand per customer), the solutions using constellation balloon configurations (carrying microLTE BSs using $20 \mathrm{MHz}$ and $40 \mathrm{MHz}$ spectrum) have the lowest costs in comparison to terrestrial LTE $20 \mathrm{MHz}$ and LTE $40 \mathrm{MHz}$. In these years balloons using either 
$20 \mathrm{MHz}$ or $40 \mathrm{MHz}$ are able to serve the customer and speed demand in both study areas.

In years 6 to 7 (4Mbps average broadband speed demand per customer), balloon solutions using LTE 40MHz become the lowest cost option. Balloons using LTE $20 \mathrm{MHz}$ switch to terrestrial solutions in the Yauli area (terrestrial LTE $20 \mathrm{MHz}$ and LTE $40 \mathrm{MHz}$ ), shown as a steep cost increase due to the terrestrial deployments in Yauli.

In year 8 (8Mbps average broadband speed demand per customer), balloons using LTE $40 \mathrm{MHz}$ remain the lowest cost option, although its cost shows a steep increase due to terrestrial deployments in Yauli. Balloons using LTE 20MHz also shows a steep cost increase due to terrestrial deployments in Samugari.

In years 9 to $10(8 \mathrm{Mbps}$ and $12 \mathrm{Mbps}$ average broadband speed demand per customer), balloon solutions with both LTE $20 \mathrm{MHz}$ and LTE $40 \mathrm{MHz}$ are operating terrestrial networks LTE $20 \mathrm{MHz}$ or LTE $40 \mathrm{MHz}$.

Based on these cumulative costs, balloons using LTE $20 \mathrm{MHz}$ and LTE $40 \mathrm{MHz}$ present a significant lower initial investment for the first years in comparison to the terrestrial options (LTE $20 \mathrm{MHz}$ and $40 \mathrm{MHz}$ ), as follows:

- Years 1 to 5: The cumulative costs of balloons using either LTE $20 \mathrm{MHz}$ and LTE $40 \mathrm{MHz}$ ranges from $\mathbf{3 6} \%$ (year 1) to $\mathbf{6 1 \%}$ (year 5) of cumulative costs of terrestrial networks using either LTE $20 \mathrm{MHz}$ or LTE $40 \mathrm{MHz}$.

- Years 6 to 7: The cumulative costs of balloons using LTE $40 \mathrm{MHz}$ ranges from $63 \%$ (year 6) to $\mathbf{6 9 \%}$ (year 7) of using terrestrial LTE $40 \mathrm{MHz}$. The 
cumulative costs of balloons using LTE $20 \mathrm{MHz}$ ranges from $\mathbf{7 2} \%$ (year 6) to $\mathbf{7 6} \%$ (year 7) of using terrestrial LTE $20 \mathrm{MHz}$.

- Year 8: The cumulative costs of balloons using LTE $40 \mathrm{MHz}$ is around $\mathbf{8 1 \%}$ of using terrestrial LTE 40MHz. The cumulative costs of balloons using LTE $20 \mathrm{MHz}$ become higher than terrestrial LTE $20 \mathrm{MHz}$.

- Years 9 to 10: The cumulative costs of balloons using LTE $40 \mathrm{MHz}$ become higher than terrestrial LTE $40 \mathrm{MHz}$.

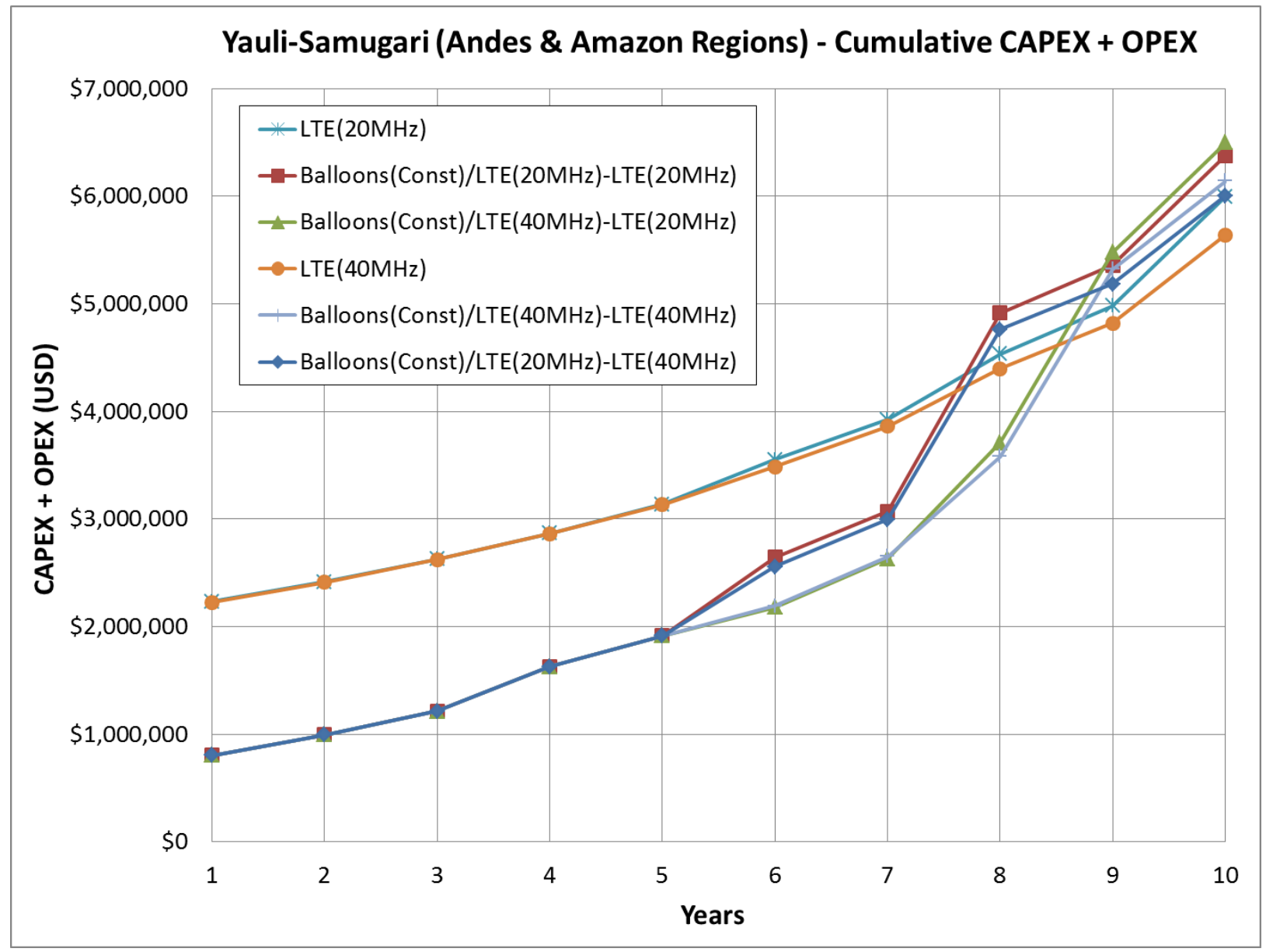

Figure 59. Cumulative CAPEX \& OPEX of the combined (Yauli and Samugari) deployment and operation costs of roadmap technology options 
These initial lower investments using balloons can be a strong incentive for broadband service providers to serve these rural areas with a lower investment risk. Additionally, if demand over the first years of service does not take off, there is the chance to relocate the balloons coverage or flight route to another area. That is investment would not be lost (as in the case of fixed terrestrial network infrastructure) if ceasing operations in a specific rural area.

In conclusion, we propose that the technology roadmap solution to introduce broadband services in underserved and unserved areas in the Andes and Amazon regions of Peru consists of WBLANs using the following two solutions:

1. Stratospheric balloons carrying micro-LTE base stations using $20+20 \mathrm{MHz}$ for the first years and then deploying terrestrial LTE $40+40 \mathrm{MHz}$.

2. Stratospheric balloons carrying micro-LTE base stations $40+40 \mathrm{MHz}$ for the first years and then deploying terrestrial LTE $40+40 \mathrm{MHz}$.

This technology roadmap can be implemented if the following points are taken by telecom regulators and broadband service providers in Peru:

- The government regulators, MTC and OSIPTEL, allows using unmanned stratospheric balloons to provide broadband service in underserved and unserved rural areas in the Andes and Amazon regions in Peru. UAS regulation in Peru to provide broadband services is in an early stage but based on stratospheric balloon flight tests already conducted in Peru [110] [111] [112], we assume that the Peruvian telecom regulators must be 
evaluating the use of stratospheric balloons to provide service in remote rural areas. The aviation administration agency, in late 2015, approved and set regulations for the flight of free unmanned balloons in Peruvian air space [113] [114]. This year, Project Loon announced tests and discussions with the regulator and operators in Sri Lanka and India for the deployment and operation of the stratospheric balloons to provide broadband services [56] [58] [59] [102].

- Broadband service providers work together with the Project Loon in order to integrate operators' business with the innovative stratospheric platforms designed by Google. Broadband service providers have already the expensive LTE spectrum and also a mature business already deployed in the Peru. Project Loon can provide the stratospheric platforms operated with cuttingedge technology which senses winds patterns in the stratosphere to determine the route of a single or a constellation of balloons to fly over specific areas to provide broadband services. By providing these services, broadband services providers benefit from new customers which otherwise would have not been able to reach without significant infrastructure investments, Google benefit from new Internet users and potential new customers.

- The regulators (MTC and OSIPTEL) lift the current 1.7/2.1GHz LTE band spectrum cap for rural areas. For the initial years, balloons using LTE either $20+20 \mathrm{MHz}$ or $40+40 \mathrm{MHz}$ provide the lowest cost as long as the 
complementary terrestrial network is LTE $40+40 \mathrm{MHz}$. Then the solutions proposed for this broadband technology roadmap relies on allowing broadband service providers to use this spectrum at least in rural areas. This technology roadmap assumes that, based on both current stratospheric balloons flight tests and propagation analysis of using micro-LTE base stations $20 \mathrm{~km}$ above the ground, Project Loon's balloons might work as expected to provide broadband services to rural areas in developing countries. However, as in the case of many disruptive and innovative technologies, the first commercial services using stratospheric balloons might require several iterations in several aspects such as balloons altitude control system and flight patterns, interaction within the commercial aviation airspace when ascending or descending, roaming and handover from micro-LTE base stations, backhaul radio link alignment, business model, spectrum allocation, among other areas. 


\section{CHAPTER VI}

\section{DISCUSSION AND SUMMARY}

\subsection{Hypotheses results and findings}

Hypothesis 1: Deploying wireless access networks in the Andes and Amazon regions of Peru using the Wi-Fi technology and unlicensed spectrum has the lowest deployment and operating cost in a 10-year period.

We tested this hypothesis by conducting technical and engineering cost analyses in the two study areas (Yauli County in the Andes Region and Samugari County in the Amazon region) to estimate the cost of deploying and operating terrestrial wireless access networks employing current wireless technologies (i.e. Wi-Fi, WiMAX, LTE and TV White Spaces) and new stratospheric wireless access networks (i.e. using stratospheric balloons) carrying micro-LTE base stations.

The engineering cost analysis results showed that, in both study areas, in the cumulative deployment and operation CAPEX and OPEX (shown in Figure 27 and Figure 28) at deployment (year 1), Wi-Fi has the highest initial cost due to requiring the most number of nodes. Initially, Wi-Fi requires more nodes than the other technologies due to its limited channel access mechanism to serve large number of CPEs at distances of more than a kilometer (which requires to activate 
additional collision avoidance features which increases the overhead and leads to a decrease in the effective download channel capacity), and the requirement of LOS or near-LOS between APs and CPEs. Wi-Fi was originally designed for WLANs to enable communication among devices in a range from tens to a few hundred meters. During years 4 to 7, in Yauli, Wi-Fi's cumulative costs come closer to WiMAX costs, and in Samugari, Wi-Fi costs remain the highest. From years 8 to 10, in both study areas, Wi-Fi's costs becomes lower than TVWS and WiMAX; however, LTE remains the lowest cost option and has the lowest cumulative cost in year 10 .

Based on the net present value (NPV) applied to the yearly CAPEX and OPEX over the 10-year period (shown in Figure 29), LTE has the lowest NPV in both study areas. In Yauli, the NPV of Wi-Fi, WiMAX and TVWS is 13\%, 29\% and $59 \%$ more expensive than LTE, respectively. In Samugari, the NPV of Wi-Fi, WiMAX and TVWS is $12 \%, 27 \%$ and $30 \%$ more expensive than LTE, respectively. It is also important to note that the NPV for all analyzed technologies in Samugari is higher than in Yauli due to the longer distances among towns and the dense vegetation, which required using more nodes and also taller towers (more expensive infrastructure cost) to enable line-of-sight for the backhauls above the jungle canopy.

The breakdown of the CAPEX NPV shows that, in the Yauli area (shown in Figure 30), LTE presents the lowest total CAPEX. In the aggregated categories of towers, civil engineering and energy system (network node infrastructure cost), 
LTE has the lowest cost, but in the combined network equipment (APs and backhaul equipment) and CPE categories, Wi-Fi has the overall lowest cost. In the Samugari area, LTE also presents the lowest CAPEX. In the aggregated categories of towers, civil engineering and energy system (network node infrastructure cost), Wi-Fi has the highest cost, but in the combined network equipment and $\mathrm{CPE}$ categories, Wi-Fi has the lowest cost. In both study areas, the higher infrastructure cost of Wi-Fi outweighs the low cost of Wi-Fi equipment achieved due to economies of scale of this technology. On the other hand, the lower infrastructure costs of LTE combined with the mid-range cost of LTE equipment results in the lowest cost option in both study areas.

One important aspect of Wi-Fi, which in many cases is indicated as a drawback, is the use of unlicensed spectrum. It has been pointed out that overusing unlicensed spectrum can lead to spectrum saturation and, as a result, degradation of the quality of service may occur. However, in WBLANs using Wi-Fi in rural areas in the Peruvian Andes or Amazon regions, it is not expected to face significant interference from other Wi-Fi devices outside the ones (APs and CPEs) considered in our WBLAN designs. Additionally, for Wi-Fi and also for the case of the other technologies, our technical designs included an interference analysis of APs and CPEs using the same channel, and results showed that the networks do not have significant co-channel interference. In the case of LTE, this technology uses licensed spectrum in Peru. The cost model in this analysis does not include the cost of the nationwide licensed LTE bands. The analysis assumes that some 
broadband service providers already have acquired these LTE licensed bands for operations in the largest mobile markets in Peru, giving these service providers the ability to also expand operations in rural areas.

Based on our technical and engineering cost analyses, LTE comes as the lowest cost solution for rural areas in the Peruvian Andes and Amazon regions under current spectrum regulations. Then the hypothesis that the Wi-Fi technology and unlicensed spectrum has the lowest deployment and operating cost in a 10 -year period is false.

Hypothesis 2: Wireless access networks using stratospheric platforms (i.e. stratospheric balloons and unmanned aerial vehicles) in the Andes and Amazon regions of Peru has the lowest deployment and operating cost for the initial years but are not able to scale for a 10-year period to serve forecasted customer and speed growth.

We tested this hypothesis by conducting technical and engineering cost analyses in the two study areas to estimate the cost of deploying and operating terrestrial wireless access networks employing current wireless technologies and also new stratospheric platforms such as balloons carrying micro-LTE base stations.

Our technical analysis showed that, under current Peruvian 1.7/2.1GHz LTE band spectrum regulations stratospheric balloons (using one balloon in the Yauli area and two balloons in the Samugari area) can only provide broadband service 
for five years in the Yauli area (shown in Figure 25) and for seven years in the Samugari area (Figure 26). This is due to capacity and spectrum constraints generated by the spectrum cap of $20+20 \mathrm{MHz}$ per operator set on the $1.7 / 2.1 \mathrm{GHz}$ LTE band. However, during those years, stratospheric balloons present the lowest cumulative deployment and operating costs in both study areas (shown in Figure 27 and Figure 28).

Since a technology solution of using only stratospheric balloons is not able to serve the customer and speed demands in both study areas for the 10-year period, despite providing the lowest total cumulative costs during the first years of operation, then our final proposed broadband technology roadmap included using stratospheric balloons for the initial years of the 10-year period, and then deploying complementary terrestrial WBLANs. Some of the solutions analyzed for the technology roadmap included the scenario of lifting the $1.7 / 2.1 \mathrm{GHz} \mathrm{LTE}$ spectrum band cap of $20+20 \mathrm{MHz}$ per operator and allowing operators to use a $40+40 \mathrm{MHz}$ spectrum. This scenario would impact both the stratospheric balloons and terrestrial networks using LTE. In this scenario, stratospheric balloons using micro-LTE base stations would be able to provide broadband service, in the Yauli area, for the seven years, and, in the Samugari area, for eight years (shown in Figure 57). After stratospheric balloons' micro-LTE base stations reach its maximum capacity, complementary terrestrial networks are deployed to continue providing the service for the rest of the 10-year period. At the end, the broadband technology roadmap in this study was narrowed down to two solutions: 
- Stratospheric balloons using LTE $(20+20 \mathrm{MHz})$ for the first three years in both study areas, and then deploying terrestrial LTE $(40+40 \mathrm{MHz})$ WBLANs for the rest of the 10-year period.

- Stratospheric balloons using LTE $(40+40 \mathrm{MHz})$ for the first four years in the Yauli area, and for six years in the Samugari area, and then deploying terrestrial LTE $(40+40 \mathrm{MHz})$ WBLANs for the rest of the 10-year period. For the proposed technology roadmap to work in Peru in coming years, assuming that Project Loon makes the stratospheric balloons operate as expected, three additional points need to be worked out: 1) the Peruvian telecom regulators (MTC and OSIPTEL) and aviation administration authorize using stratospheric balloons to provide broadband service in unserved and underserved areas in the Andes and Amazon regions of Peru, 2) Broadband service providers and Project Loon work collaborative to develop an innovative business model leveraging their valuable assets, and 3) the $1.7 / 2.1 \mathrm{GHz}$ LTE band spectrum cap is lifted at least to provide broadband service in unserved and underserved areas.

Since stratospheric balloons have neither launched a commercial wireless Internet service yet nor discussions with telecom regulators have disclosed specific agreements yet, except that Sri Lanka's government will have a 15 percent stake participation in the joint venture for dedicating spectrum to provide Internet service using stratospheric balloons [56] [58] [102], there is currently not telecom regulatory framework that can work as reference. Regulations for using stratospheric balloons to provide broadband Internet service will come from two 
fields: 1) aviation regulations, and 2) telecommunications regulations. The Peruvian Aviation Administration has recently set regulations for flying unmanned free balloons [113] [114] along with other countries in Latin America such as Mexico [115], Argentina [116] and Ecuador [117]. These regulations follow the same aviation regulations set by the Federal Aviation Administration of the United States in January 2015 regarding unmanned free balloons (14 CFR Part 101-Moored balloons, kites, amateur rockets and unmanned free balloons) [118], and cover the main following points: applicability, operating limitations, equipment and marking requirements, notice requirements and balloon position reports. In October 2016, Project Loon announced in a press release that they conducted balloons flight tests over Peruvian airspace [113] [114]. Before this press release, since May 2016, we followed these fly tests over Peru using air traffic control (ATC) database websites [119] which recorded the flights of two Project Loon's balloons marked as HBAL273 and HBAL305. We used the flight pattern data collected from these ATC database websites to conduct our technical and engineering cost analyses following two defined Project Loon's balloons flight patterns: 1) flying over a specific area in Peru, and 2) flying over a route in South America. Our analyses included the economics associated to each pattern. Up to date, the Project Loon has not announced yet any discussion with telecom regulators or potential joint ventures with local broadband service providers in Peru. This would be the next step in order to launch the broadband Internet service using stratospheric balloons. 
Then the hypothesis that WBANs using stratospheric platforms has the lowest deployment and operating cost for the initial years but are not able to scale for a 10-year period to serve forecasted customer and speed growth is true.

\section{Hypothesis 3: Spectrum management and regulation have a quantifiable} technology and economic impact in the deployment and operation of wireless access networks in the Andes and Amazon regions of Peru and are important in defining the best technology fit to introduce broadband services in these regions.

We tested this hypothesis by proposing spectrum management and regulation scenarios for the spectrum used by the different technology options and, by using our engineering cost model, which evaluated the impact of spectrum management and regulation on the total deployment and operating cost over a 10year period.

Our technical and engineering cost analyses of wireless broadband technologies were based on current Peruvian spectrum regulations which included the following:

- Wi-Fi: $2.4 \mathrm{GHz}$ using 3 non-interference channels of $20 \mathrm{MHz}$.

- WiMAX: $3.5 \mathrm{GHz}$ using 5 channels of $10 \mathrm{MHz}$ (spectrum cap of $50 \mathrm{MHz}$ per operator).

- LTE: $1.7 / 2.1 \mathrm{GHz}$ using 2 channels of $10+10 \mathrm{MHz}$ (spectrum cap of $20+20 \mathrm{MHz}$ FDD per operator). 
- TVWS: 470-698MHz. There is currently no TVWS regulation in Peru. We assumed that the regulator would allow using up to 15 channels in rural areas out of the 37 available digital television channels of $6 \mathrm{MHz}$.

The technical analysis showed that, in Yauli, LTE has the highest effective download channel capacity (see Table 2), and, in Samugari, LTE also has the highest effective download channel (see Table 3). LTE has a high effective download channel capacity due to its wide channel, $20 \mathrm{MHz}$ (double than LTE's) and also due to its high data rates and more throughput efficiency (less overhead) than the other technologies. In metrics regarding capacity (users and Mbps) per $\mathrm{MHz}$ in the download spectrum, LTE also provided the most capacity. At the end, in both study areas, LTE required the least number of AP/BSs due to having the highest effective download channel capacity, and also required the lowest number of nodes (infrastructure costs) due to having the highest capacity per node. Under these conditions, based on the NPV of the WBLAN deployment and operations over a 10-year period, in both study areas, LTE is the overall lowest cost option. In both study areas, Wi-Fi and WiMAX come as the second and third lowest cost options, respectively. We also analyzed the scenario of LTE using two $20+20 \mathrm{MHz}$ channels, that is lifting the $20+20 \mathrm{MHz}$ spectrum cap per operator. In the case of Wi-Fi and WiMAX, there is not more additional available spectrum in the $2.4 \mathrm{GHz}$ and 3.5GHz bands, respectively, and in the case of TVWS, we already included using a significant amount of the digital broadcast spectrum. In the case of LTE, there is a 
reserved $20+20 \mathrm{MHz}$ block in the $1 / 7 / 2.1 \mathrm{GHz}$ band to be auctioned in the coming years.

Based on the technical analysis of using LTE $40+40 \mathrm{MHz}$, this option has the highest effective download channel capacity and capacity (users and Mbps) per node (shown in Table 12 and Table 13). Based on the engineering cost analysis, this option came as the lowest cost option (shown in Figure 47 and Figure 48). LTE $40+40 \mathrm{MHz}$ was also included in the proposed broadband technology roadmap, which concluded that the best technology fit to introduce broadband services in rural areas in the Andes and Amazon regions was stratospheric balloons using LTE $(20+20 \mathrm{MHz}$ or $40+40 \mathrm{MHz})$ for the first years, in both study areas, and then deploying terrestrial LTE $(40+40 \mathrm{MHz})$ WBLANs for the rest of the 10-year period.

Our analysis showed that spectrum regulation such as spectrum caps and available channel per operator has a direct impact on the capacity per node and as a result in the overall cost of infrastructure, and total WBLAN deployment and operating costs, which can be quantified and compared. In our study, spectrum regulation analysis was used, first, to quantify technical and cost efficiencies for different technologies, and second, to determine the best technology fit or broadband technology roadmap to introduce broadband services to unserved and underserved rural areas in the Peruvian Andes and Amazon regions.

Then the hypothesis that spectrum management and regulation have a quantifiable technology and economic impact in the deployment and operation of 
WBANs and are important in defining the best technology fit to introduce broadband services is true.

\subsection{Summary}

The first chapter of the dissertation introduces the context of our research topic and the research question: What is the roadmap for introducing broadband services to unserved and underserved areas in the Andes and Amazon Regions of Peru? The hypotheses, along with the delimitations, limitations, and importance of the study are also presented. This introduction sets the framework for the research conducted in this study to answer the research question and the methodology applied to address the hypotheses.

The second chapter presents the literature review which includes the current broadband landscape in Latin America and Peru, broadband Internet penetration in Peru, and the Peruvian national broadband plan. It also covers a review of current wireless broadband technologies deployed for rural areas around the world (i.e. Wi-Fi, WiMAX, LTE, TVWS, Gigabit satellite and millimeter-wave) and new unmanned aerial systems (balloons and drones) proposed for the deployment of stratospheric access networks. At the end, this chapter also presents an overview of the regulatory and policy environment for broadband services in Peru which includes the national broadband plan's deployment of fiber optic backbone network and transport and access networks, along with current spectrum regulations for wireless broadband technologies. 
In the third chapter, we describe the detailed research methodology used to address the research question and hypotheses. The research methodology consisted of conducting quantitative technical and engineering cost analyses to estimate and compare the costs of deploying and operating WBLANs using current wireless broadband technology options and stratospheric platforms to provide broadband services in the Andes and Amazon regions of Peru. This chapter also presents the selection of the study areas (the Yauli county in the Andes region and Samugari county in the Amazon region) and access network coverage (towns within these selected counties), the estimation of broadband fixed residential demand (9.1\% of households in Samugari with a $2.9 \%$ annual growth, and $4.7 \%$ of households in Yauli with a 2.7\% annual growth) and speed demand (2Mbps from years 1 to 3 , $4 \mathrm{Mbps}$ for years 4 to $7,8 \mathrm{Mbps}$ for year 8 to 9 , and $12 \mathrm{Mbps}$ for year 10 ), and a review of spectrum resources (available spectrum in the Wi-Fi $2.4 \mathrm{GHz}$, WiMAX 3.5GHz, TVWS 470-698MHz and LTE $1.7 / 2.1 \mathrm{GHz}$ bands), which altogether work as inputs for the technical and engineering cost analyses.

The fourth chapter presents the technical and engineering cost analyses results for both study areas. The technical analysis results include the effective download channel capacity and capacity (Mbps and users) per node for the different technologies, and also the network elements (nodes, towers, AP/BSs, backhaul radios and CPEs) required for the deployment and operation of WBLANs for a 10-year period using the wireless technologies and stratospheric platforms. This technical analysis shows that LTE has the highest effective download channel 
capacity and the highest node capacity, and also showed that LTE required the least number of AP/BSs due to its high channel capacity, and also required the least number of nodes due to its high node capacity. The engineering cost analysis results shows cumulative deployment and operating costs for the WBLANs designed in the technical analysis for both study areas. It presents the net present value (NPV) of the yearly CAPEX and OPEX for the WBLANs using different wireless technologies, and also presents the CAPEX and OPEX NPV breakdown. LTE presents the lowest NPV in both study areas, making this technology the best technology fit for both study areas based on current spectrum regulations. The broadband service cost for the Yauli and Samugari areas are also presented as the cost per user per month and the cost per Mbps per month. At the end of this chapter, a sensitivity analysis of the total NPV, based on variations of the main CAPEX and OPEX categories, is also presented. The sensibility analysis shows that after a $10 \%$ cost increase in the main CAPEX and OPEX categories, LTE remains the lowest cost option based on the NPV in the two study areas.

The fifth chapter presents the regulatory and policy implications and the proposed broadband technology roadmap to introduce broadband services to unserved and underserved rural areas in the Peruvian Andes and Amazon regions. Technical and engineering cost analyses for deploying and operating WBLANs using LTE with $40+40 \mathrm{MHz}$ spectrum is presented; this scenario assumes that the $20+20 \mathrm{MHz}$ spectrum cap for the $1.7 / 2.1 \mathrm{GHz}$ LTE band is lifted. The analyses results show that under this scenario and based on the NPV, LTE $40+40 \mathrm{MHz}$ 
comes as the new lowest cost option. This chapter also presents an analysis of the subsidy provided by the FITEL bids for the deployment and operation of broadband networks in the states of Huancavelica and Ayacucho, wherein our study areas are located. The analysis shows that, based on the WBLAN estimated costs in the two study areas, the FITEL subsidy in Huancavelica allows for at least 58\% percent profit (if LTE is used), and the FITEL subsidy in Ayacucho in addition to a USD 12.5 broadband service monthly fee allows the broadband service provider to break even. At the end of this chapter, we present our broadband technology roadmap which includes using stratospheric balloons carrying micro-LTE base stations (using $20+20 \mathrm{MHz}$ or $40+40 \mathrm{MHz}$ spectrum) for the first years, in both study areas, and then deploying terrestrial LTE $(40+40 \mathrm{MHz})$ WBLANs for the rest of the 10year period.

\subsection{Conclusions}

By addressing the three hypotheses, we answer the research question What is the roadmap for introducing broadband services to unserved and underserved areas in the Andes and Amazon Regions of Peru? Our broadband technology roadmap comes as a result of conducting technical and engineering cost analyses for deploying and operating WBLANs using current wireless broadband technologies (Wi-Fi, WiMAX, TVWS and LTE) and innovative unmanned platforms (stratospheric balloons) designed to fly in the lower stratosphere $20 \mathrm{~km}$ above the ground. Our analyses were based on current spectrum regulations and additional 
proposed spectrum scenarios which allowed evaluating the most efficient technologies currently working under spectrum constraints, such as the case of 1.7/2.1GHz LTE. Under current spectrum regulations and based on the NPV, LTE is the lowest cost option for WBLANs in both the Andes and Amazon regions. Under the scenario of lifting the 1.7/2.1GHz LTE band spectrum cap, LTE $40+40 \mathrm{MHz}$ comes as the lowest cost option. Furthermore, when combining using stratospheric balloons, carrying micro-LTE base stations, with complementary terrestrial LTE deployments using $40+40 \mathrm{MHz}$ spectrum, the lowest and most innovative solution is provided. Using stratospheric balloons (either using single balloon or constellation configurations) allows fast and low cost initial deployment and operation for at least five years, or up to eight years, and then complementary terrestrial LTE networks are requires for the rest of the 10-year period. This is the broadband technology roadmap proposed in this study.

In the 2015 Telecom Policy Research Conference (TPRC 43), held in Arlington VA, the plenary panel session Industry as an Audience for Academic Policy Research addressed the much need for data based proposed policies and regulations in the telecom arena. Our rigorous quantitative technical and engineering cost analyses allowed us to propose this type of data based regulations and policies to further the expansion of broadband services to underserved and unserved areas in developing countries. Our study is also able to integrate technology, economics, and regulation and policy aspects of broadband for currently underserved and unserved areas; seen as emerging markets which might evolve 
into profitable markets based on the potential customer volume in the future, or "the next 5 billion" by 2030 . 


\section{Bibliography}

[1] National Institute for Statistics and Informatics (INEI), "Health Statistics 2012," [Online]. Available: http://www.inei.gob.pe/estadisticas/indicetematico/health/. [Accessed March 2014].

[2] National Institute for Statistics and Informatics (INEI), "Technical report: Monetary poverty evolution 2007-201," [Online]. Available: http://www.inei.gob.pe/media/cifras_de_pobreza/pobreza_informetecnico2013_ 1.pdf.

[3] H. Galperin, J. Mariscal and F. Viecens, "An analysis of national broadband plans in Latin America," Economics Research and Teaching Center: Work document. Mexico DF, Mexico, vol. 274, 2012.

[4] Investment Fund in Telecommunications (FITEL), "National fiber optic backbone project," [Online]. Available: http://www.fitel.gob.pe/pg/proyectosformulacion.php. [Accessed March 2014].

[5] Supervisory Agency of Private Investment in Telecommunications (OSIPTEL), "Internet Subscribers by Service Type per State: 2011-2012," 2013. [Online]. Available: http://www.osiptel.gob.pe. [Accessed March 2014].

[6] H. Galperin and B. Girard, "Microtelcos in Latin America and the Caribbean," in Report on the World Dialogue on Regulation: Diversifying participation in network development-Case studies, vol. Research Cycle 3, World Dialogue on Regulation for Network Economies, 2007.

[7] D. Reed, J. Haroon and P. Ryan, "Technologies and policies to connect the next 5 billion," Berkeley Technology Law Journal, vol. 29, 2014.

[8] World Bank, Information and Communications for Development : Extending Reach and Increasing Impact, World Bank Press, 2009.

[9] S. Buttkereit, L. Enriquez, F. Grijpink, S. Torfs and T. Vaheri-Delmulle, "Mobile broadband for the masses: Regulatory levers to make it happen. Report McKinsey \& Company," McKinsey \& Company, 2009.

[10] R. Friedrich, K. Sabbagh, B. El-Darwiche and M. Singh, "Digital Highways: The Role of Government in 21st-Century Infrastructure," Strategy (Formerly Booz \& Company), 2009.

[11] Latin American Asociation of Research Centers and Companies in Telecommunications (AHCIET), "AHCIET Statement - Sao Paulo," May 2010. [Online]. Available: http://www.itu.int/ITU- 
D/conferences/wtdc/2010/policystatements/speeches/AHCIET_garcia_vf_es.pdf . [Accessed March 2014].

[12] Supervision Agency of Private Investment in Telecommunications (OSIPTEL); Pacheco, Luis, "National Plan Proposal for Broadband Development in Peru. OSIPTEL's Role and Learned Lessons. Seminar on Economic and Financial Aspects of Telecommunications-SG3RG-LAC-ITU," Study Group 3 Regional Group for Latin America and the Caribbean - ITU, San Salvador, 2011.

[13] Latin American Asociation of Research Centers and Companies in Telecommunications (AHCIET), "Broadband Plans in Latin America," AHCIET, Montevideo, 2013.

[14] Minisitry of Transportation and Communications of Peru, "National Plan for the Development of Broadban in Peru," MTC, 2010.

[15] Supervision Agency of Private Investment in Telecommunications (OSIPTEL), "Statistics Indicators: Fixed and Mobile Internet Access".

[16] Supervisory Agency of Private Investment in Telecommunications (OSIPTEL), "Characterization of Fixed and Mobile Internet Demand in Peru: 2012-2013," 2014.

[17] Ministry of Transportation and Telecommunications, "Nationwide Fiber Optic Backbone," 2014.

[18] Investment Fund in Telecommunications (FITEL), "Current Projects".

[19] Investment Fund in Telecommunications (FITEL), "Projects in Planning Stage".

[20] V. Gunasekaran, "Emerging Wireless Technologies for Developing Countries," Technology in Society, vol. 29, pp. 23--42, 2007.

[21] F. Proenza, "The Roadmap to Broadband Development in Developing Countries Is through Competition Driven by Wireless and Internet Telephony," Information Technologies and International Development, vol. 3(2), pp. 21--39, 2006.

[22] F. Simba, "Broadband Access Technologies for Rural Connectivity in Developing Countries," International Journal of Research and Reviews in Computer Science (IJRRCS), vol. 2(2), 2011.

[23] T. Schwengler, Wireless \& Cellular Communications - Class Notes for TLEN5510, 2014.

[24] R. Bhaskaran and C. Kameswari, "Design and evaluation of a new MAC protocol for long-distance 802.11 mesh networks," in Proceedings of the 11th annual international conference on Mobile computing and networking, 2005. 
[25] R. Patra, S. Nedevschi, S. Surana, A. Sheth, L. Subramanian and E. Brewer, "WiLdnet Design and implementation of high performancewifi based long distance networks," in Proceedings of the 4th USENIX conference on Networked systems design and implementation, 2007.

[26] B. Singh, "A High Performance Point-to-Point Rural Wi-Fi and Wi-MAX Access Networks in Developing Regions," in NETAPPS '10 Proceedings of the 2010 Second International Conference on Network Applications, Protocols and Services, 2010.

[27] F. Simo-Reigadas, F. Martinez and C. Figuera, "Distance Limits in IEEE 802.11 for Rural Networks in Developing Countries," in Conference on Wireless Rural and Emergency Communications (Wrecom 2007), 2007.

[28] AirJaldi Networks, "AirJaldi Networks," [Online]. Available: http://www.http://main.airjaldi.com/. [Accessed May 2015].

[29] Nepal Wireless, "Nepal Wireless," [Online]. Available: http://www.nepalwireless.net/. [Accessed May 2015].

[30] C. Rey-Moreno, I. Bebea-Gonzalez, I. Foche-Perez, R. Quispe-Tacas, L. LinanBenitez and J. Simo-Reigadas, "A Telemedicine Wi-Fi Network Optimized for Long Distances in the Amazonian Jungle of Peru," in IEEE ExtremeCom Proceedings of the 3rd Extreme Conference on Communication - The Amazon Expedition, 2011.

[31] M. Murillo, The Peru Experience, IEEE Sections Congress 2011, 2011.

[32] Iberbanda, "Iberbanda," [Online]. Available: http://www.iberbanda.com/LaCompania. [Accessed May 2015].

[33] WiMAX Forum, "WiMAX Provides Essential Broadband Services in Suburban and Rural Territories in Spain," WiMAX Forum, 2008. [Online]. Available: http://www.wimaxforum.org/sites/wimaxforum.org/files/document_library/iber banda.pdf. [Accessed May 2015].

[34] WiMAX Forum, "WiMAX, a Wireless Solution for Fixed Wireless Access in Emerging Markets," WiMAX Forum, April 2010. [Online]. Available: http://resources.wimaxforum.org/sites/wimaxforum.org/files/document_library/ DAP0410_WIMAX\%20A\%20WIRELESS\%20SOLUTION\%20FOR\%20FIXED \%20WIRELESS\%20ACCESS\%20IN\%20EMERGING\%20MARKETS.pdf. [Accessed May 2015].

[35] Packet One (P1), "P1 Completes World's First 2.3GHz WiMAX Community Broadband Centres," Packet One (P1), May 2010. [Online]. Available: http://www.p1.com.my/pressreleases/25052010_P1_Completes_World_First_2. 3GHz_WiMAX_Community_Broadband_Centres.htm. [Accessed May 2015]. 
[36] Washington Post, "Remote Vietnamese village gets Internet access via WiMax," 21 September 2007. [Online]. Available:

http://www.washingtonpost.com/wpdyn/content/article/2007/09/21/AR2007092100489.html. [Accessed May 2015].

[37] C. F. Gomes and M. P. Fernandez, "Infrastructure and Business Model for Universal Broadband Access in Developing Regions: The Ceara State Digital Belt," in Third IFIP TC 6 International Conference, WCITD 2010 and IFIP TC 6 International Conference, NF 2010, Held as Part of WCC 2010, 2010.

[38] L. V. Souza and C. R. Frances, "Integrated Solutions for Broadband Access in Brazilian Amazon Rural Areas," in IEEE Globecom Workshops, Anaheim, CA, 2012.

[39] Advanced Wireless Technology, "AWT Global - LTE E-UTRAN, 3GPP Frequency Bands," [Online]. Available: http://www.awtglobal.com/resources/lte-e-utran-bands/. [Accessed may 2015].

[40] GSMA Intelligence, "Mobile broadband reach expanding globally: New GSMA Intelligence data set on mobile broadband coverage," December 2014. [Online]. Available: https://gsmaintelligence.com/research/2014/12/mobilebroadband-reach-expanding-globally/453/. [Accessed May 2015].

[41] Dish Networks, "Press Release: DISH and nTelos Wireless Offer Fixed Internet Service To Customers in Virginia," Dish Networks, 16 July 2014. [Online]. Available: http://about.dish.com/press-release/dish-and-nteloswireless-offer-fixed-internet-service-customers-virginia. [Accessed May 2015].

[42] Dish Networks, "Press Release: DISH Delivers High-Speed LTE Internet Service in Corpus Christi," Dish Networks, 24 September 2014. [Online]. Available: http://about.dish.com/press-release/dish-delivers-high-speed-lteinternet-service-corpus-christi. [Accessed May 2015].

[43] Ericsson, "News Center: LTE to bring fixed-wireless broadband to rural Australia," Ericsson, 1 June 2011. [Online]. Available: http://www.ericsson.com/news/1520376. [Accessed May 2015].

[44] Business Wire, "Parallel Wireless Reimagines Rural Cellular Coverage," Business Wire, 1st December 2014. [Online]. Available: http://www.businesswire.com/news/home/20141201006542/en/ParallelWireless-Reimagines-Rural-Cellular-Coverage\#.VV5tj0_BzRY. [Accessed May 2015].

[45] Nokia, "Avantel, Nokia Networks to deploy VoLTE and LTE small cells in Colombia," Nokia, 15 March 2015. [Online]. Available: http://company.nokia.com/en/news/press-releases/2015/03/03/avantel-nokianetworks-to-deploy-volte-and-lte-small-cells-in-colombia. [Accessed May 
2015].

[46] E. Pietrosemoli and M. Zennaro, TV White Spaces - A Pragmatic Approach, ICTP-The Abdus Salam International Centre forTheoretical Physics T/ICT4D Lab, 2013.

[47] Google, "Spectrum Database," Google, [Online]. Available: https://www.google.com/get/spectrumdatabase/.

[48] T. Brown, E. Pietrosemoli, M. Zennaro, A. Bagula, H. Mauwa and S. Nleya, "A survey of TV White Space Measurements," in e-Infrastructure and e-Services for Developing COuntries - 6th International COnference (AFRICOMM 2014, Kampala, Uganda, 24-25 November 2014), Springer International Publishing, 2015, pp. 164--172.

[49] Dynamic Spectrum Alliance, "Pilots," Dynamic Spectrum Alliance, [Online]. Available: http://www.dynamicspectrumalliance.org/pilots/. [Accessed May 2015].

[50] O3b Networks, "O3b Networks - Services," O3b Networks, 2012. [Online]. Available: http://www.o3bnetworks.com/. [Accessed May 2015].

[51] C. Dehos, J. L. Gonzalez, A. De Domenico, D. Ktenas and L. Dussopt, "Millimeter-Wave Access and Backhauling: The Solution to the Exponential Data Traffic Increase in 5G Mobile Communications Systems?," IEEE Communications Magazine, vol. 14, no. 0163-6804, pp. 88--95, 2014.

[52] Google - Google X, "Project Loon," Google - Google X, [Online]. Available: https://www.solveforx.com/loon/.

[53] M. V. C. (. Google Inc., R. W. DeVaul, E. Teller, C. L. Biffle and J. Weaver, "Balloon Power Sources with a Buoyancy Trade-Off". United States of America Patent US 2015/0168955 A1, 3 February 2015.

[54] The Verge and B. Popper, "Inside Project Loon: Google's internet in the sky is almost open for business," The Verge, 2 March 2015. [Online]. Available: http://www.theverge.com/2015/3/2/8129543/google-x-internet-balloon-projectloon-interview. [Accessed June 2015].

[55] Wired and S. Levy, "Google's Balloon Internet Experiment, One Year Later," Wired, 16 June 2014. [Online]. Available: http://www.wired.com/2014/06/google-balloons-year-later/. [Accessed June 2015].

[56] A. Pandey, "Project Loon: Google's Balloon-Powered Internet Service To Begin Tests In Sri Lanka," International Business Times, 16 February 2016. [Online]. Available: http://www.ibtimes.com/project-loon-googles-balloonpowered-internet-service-begin-tests-sri-lanka-2308605. [Accessed June 
2016].

[57] Fortune, "Google's Internet Balloons Are Arriving In Sri Lanka," Fortune, 16 February 2016. [Online]. Available: http://fortune.com/2016/02/16/google-loonsri-lanka/. [Accessed June 2016].

[58] Phys.org, "Google 'Project Loon' balloon enters Sri Lanka for Internet tests," Phys.org, 15 February 2016. [Online]. Available: http://phys.org/news/2016-02google-loon-balloon-sri-lanka.html. [Accessed June 2016].

[59] Tech Crunch, "Google in talks with telecoms to pilot Project Loon in India," Tech Crunch, 8 March 2016. [Online]. Available: https://techcrunch.com/2016/03/08/google-in-talks-with-telecoms-to-pilotproject-loon-in-india/. [Accessed June 2016].

[60] Titan Aerospace, "Titan Aerospace," March 2014. [Online]. Available: http://titanaerospace.com/. [Accessed March 2015].

[61] T. verge and N. Statt, "Google's Project SkyBender aims to beam 5G internet from solar-powered drones," The Verge, 29 January 2016. [Online]. Available: http://www.theverge.com/2016/1/29/10873676/google-project-skybenderdrones-5g-internet. [Accessed March 2016].

[62] PC World and N. Mediati, "Google's Project Skybender uses drones to bring super fast Internet to the skies," PC World, 31 January 2016. [Online]. [Accessed March 2016].

[63] Facebook, "Connectivity Lab at Facebook," Facebook, [Online]. Available: https://www.facebook.com/zuck/posts/10101322049893211. [Accessed March 2014].

[64] Wired and J. Hempel, "INSIDE FACEBOOK'S AMBITIOUS PLAN TO CONNECT THE WHOLE WORLD," Wired, 19 January 2016. [Online]. Available: https://www.wired.com/2016/01/facebook-zuckerberg-internet-org/. [Accessed June 2016].

[65] New York Times, "Facebook Moves One Step Closer to Light-Based Wireless Internet," New york Times, 21 July 2016. [Online]. Available: http://www.nytimes.com/2016/07/22/science/facebook-wireless-lightinternet.html. [Accessed July 2016].

[66] Ministry of Trasnportation and Communications of Peru, "Ministry directive to approve the National Spectrum Allocation Plan," Ministry of Trasnportation and Communications of Peru, 3 April 2005. [Online]. Available: https://www.mtc.gob.pe/portal/comunicacion/politicas/normaslegales/nuevo_P NAF_version_agosto2006.pdf. [Accessed May 2015]. 
[67] Ministry of Transportion and Communications (MTC) of Peru, "Supreme Directive No 024-2008-MTC: General Legal Framework for promotion of public telecommunication services in rural areas and places of preferred social interest - Appendix I - Title V," Ministry of Transportion and Communications (MTC) of Peru, 16 August 2008. [Online]. Available: http://www.mtc.gob.pe/portal/comunicacion/politicas/normaslegales/D.S.\%20N \%C2\%BA\%20024-2008-MTC.pdf. [Accessed May 2015].

[68] Ministry of Trasnportation and Communications (MTC) of Peru, "Ministry Directive No 324-2011-MTC/03: Modifycations to points P23, P41A, P55 and P57 and to the National Spectrum Allocation Plan in the $890-928 \mathrm{MHz}$ band, and for setting the $900 \mathrm{MHz}$ band auction," Ministry of Trasnportation and Communications (MTC) of Peru, 6 May 2011. [Online]. Available: https://www.mtc.gob.pe/portal/comunicacion/politicas/normaslegales/R\%20M\% 20324\%202011\%20MTC\%2003.pdf. [Accessed May 2015].

[69] Ministry of Transportion and Communications (MTC) of Peru, "Supreme Directive No 006-2013-MTC: Modifications to the general legal framework for promotion of public telecommunication services in rural areas and places of preferred social interest," Ministry of Transportion and Communications (MTC) of Peru, 4 April 2013. [Online]. Available: http://www.mtc.gob.pe/portal/comunicacion/politicas/normaslegales/ADECRE TO\%20SUPREMO\%20N\%20006\%202013\%20MTC0001.pdf. [Accessed May 2015].

[70] Ministry of Transportation and Communications (MTC) of Peru, "Ministry Directive No 645-2006-MTC/03: Modification of the National Spectrum Allocation Plan," Ministry of Transportation and Communications (MTC) of Peru, 18 August 2006. [Online]. Available: hhttp://www.mtc.gob.pe/portal/comunicacion/politicas/normaslegales/RM_489_ 2007_MTC.pdf. [Accessed May 2015].

[71] Ministry of Transportation and Communications (MTC) of Peru, "Ministry Directive No 489-2007-MTC/03: Modification to notes P11A of the National Spectrum Allocation Plan," Ministry of Transportation and Communications (MTC) of Peru, 27 August 2007. [Online]. Available: hhttp://www.mtc.gob.pe/portal/comunicacion/politicas/normaslegales/RM_489_ 2007_MTC.pdf. [Accessed May 2015].

[72] Ministry of Transportion and Communications (MTC) of Peru, "Ministry Directive No 317-2009-MTC/03: Modification to notes P11A of the National Spectrum Allocation Plan," Ministry of Transportion and Communications (MTC) of Peru, 23 April 2009. [Online]. Available: http://www.mtc.gob.pe/portal/comunicacion/politicas/normaslegales/R\%20M\%2 0317.PDF. [Accessed May 2015]. 
[73] Ministry of Transportation and Communications (MTC) of Peru, "Ministry Directive No 150-2010-MTC/03: Modification to notes P11A and P51 of the National Spectrum Allocation Plan and the 614-806MHz band," Ministry of Transportation and Communications (MTC) of Peru, 29 March 2010. [Online]. Available:

http://www.mtc.gob.pe/portal/comunicacion/politicas/normaslegales/3plan\%20 anual\%20de\%20mod.\%20de\%20frecuencias.pdf. [Accessed May 2015].

[74] Ministry of Transportation and Communications (MTC) of Peru, "Setting a $50 \mathrm{MHz}$ cap for spectrum assignment for public telecommunication services in the $3400-3600 \mathrm{MHz}$ band per licensee in any geographic area," Ministry of Transportation and Communications (MTC) of Peru, 21 January 2006. [Online]. Available: http://www.mtc.gob.pe/portal/comunicacion/politicas/normaslegales/DS0022006-MTC.pdf. [Accessed May 2015].

[75] Ministry of Transportation and Communications (MTC) of Peru, "Assignment of the $3.5 \mathrm{GHz}$ band won't have technology's regulation," Ministry of

Transportation and Communications (MTC) of Peru, February 2006. [Online]. Available:

http://www.mtc.gob.pe/portal/comunicacion/politicas/ingles/NP_Banda_3.5_ing les.pdf. [Accessed May 2015].

[76] Ministry of Transportation and Communications (MTC) of Peru, "Work Document: Spectrum Assignment Caps for Public Mobile Services," Ministry of Transportation and Communications (MTC) of Peru, 19 January 2012. [Online]. Available: http://www.mtc.gob.pe/PORTAL/comunicacion/politicas/proyectos/RM_015.pdf . [Accessed May 2015].

[77] Ministry of Transportation and Communications (MTC) of Peru, "National Spectrum Registry - 3400-3600MHz band," Ministry of Transportation and Communications (MTC) of Peru, May 2015. [Online]. Available: http://www.mtc.gob.pe/portal/comunicacion/concesion/registros/rnf/Bandas_34 00-3600.html.

[78] Ministry of Transportation and Communications (MTC) of Peru, "Ministry directive for modifycations to note P67 $(2,500 \mathrm{MHz})$ of the National Spectrum Allocation Plan," Ministry of Transportation and Communications (MTC) of Peru, 20 August 2007. [Online]. Available:

https://www.mtc.gob.pe/portal/comunicacion/politicas/normaslegales/RM\%204 76-2007-MTC.pdf. [Accessed May 2015].

[79] Agency for Private Investment Promotion (ProInversion) of Peru, "2,600MHz WiMAX Bands Auction Documents," Agency for Private Investment Promotion (ProInversion) of Peru, [Online]. Available: 
http://www.proyectosapp.pe/modulos/JER/PlantillaStandard.aspx?ARE=0\&PF $\mathrm{L}=2 \& J E R=4444 \& \mathrm{SEC}=22$. [Accessed May 2015].

[80] Ministry of Transportation and Communications (MTC) of Peru, "Supreme Directive to modify the spectrum assignment caps for public mobile services," Ministry of Transportation and Communications (MTC) of Peru, 31 August 2012. [Online]. Available: http://transparencia.mtc.gob.pe/idm_docs/normas_legales/1_0_3084.pdf. [Accessed May 2015].

[81] Ministry of Transportation and Communications - Comision Multisectorial Temporal, "National Plan for Broadband Development in Peru - Section 5.2.4: Spectrum Availability Barriers for Development of Mobile Broadband," Ministry of Transportation and Communications, May 2011. [Online]. Available: http://www.mtc.gob.pe/portal/proyecto_band_ancha/Plan\%20Banda\%20Ancha \%20vf.pdf. [Accessed May 2015].

[82] Ministry of Transportation and Communications (MTC) of Peru, "Ministry directive for modifycation of notes P51, P65, P67, and the National Spectrum Allocation Plan in the bands $2,110-2,120 \mathrm{MHz}, 2,120-2,160 \mathrm{MHz}$, and 2,160$2,170 \mathrm{MHz}, "$ Ministry of Transportation and Communications (MTC) of Peru, 14 March 2011. [Online]. Available:

http://www.mtc.gob.pe/portal/comunicacion/politicas/normaslegales/2R\%20M\% 20188\%202011\%20MTC\%2003\%20PNAF.pdf. [Accessed May 2015].

[83] Agency for Private Investment Promotion (ProInversion) of Peru, "899$915 \mathrm{MHz} / 944-960 \mathrm{MHz}$ bands (Lima) and $902-915 \mathrm{MHz} / 947-960 \mathrm{MHz}$ bands (rest of the country) Auction Documents," Agency for Private Investment Promotion (ProInversion) of Peru, [Online]. Available: http://www.proyectosapp.pe/modulos/JER/PlantillaPopUp.aspx?ARE=0\&PFL= 0\&JER=6149. [Accessed May 2015].

[84] Agency for Private Investment Promotion (ProInversion) of Peru, "Broadband Deployment for Integrated Connectivity and Social Development for Apurimac," Agency for Private Investment Promotion (ProInversion) of Peru, 20 August 2014. [Online]. Available: http://www.proyectosapp.pe/modulos/JER/PlantillaProyecto.aspx?ARE=0\&PF $\mathrm{L}=2 \& J E R=8021 \& \mathrm{SEC}=24$. [Accessed March 2015].

[85] Agency for Private Investment Promotion (ProInversion) of Peru, "Broadband Deployment for Integrated Connectivity amd Social Development for Ayacucho," Agency for Private Investment Promotion (ProInversion) of Peru, 20 August 2014. [Online]. Available: http://www.proyectosapp.pe/modulos/JER/PlantillaProyecto.aspx?ARE=0\&PF $\mathrm{L}=2 \& J E R=8022 \& \mathrm{SEC}=24$. [Accessed March 2015]. 
[86] Agency for Private Investment Promotion (ProInversion) of Peru, "Broadband Deployment for Integrated Connectivity and Social Development of Huancavelica," Agency for Private Investment Promotion (ProInversion) of Peru, 20 August 2014. [Online]. Available: http://www.proyectosapp.pe/modulos/JER/PlantillaProyecto.aspx?ARE=0\&PF $\mathrm{L}=2 \& J E R=8023 \& \mathrm{SEC}=24$. [Accessed March 2015].

[87] Agency for Private Investment Promotion (ProInversion) of Peru, "Broadband Deployment for Integrated Connectivity and Social Development for Lambayeque," Agency for Private Investment Promotion (ProInversion) of Peru, 20 August 2014. [Online]. Available: http://www.proyectosapp.pe/modulos/JER/PlantillaProyecto.aspx?ARE=0\&PF $\mathrm{L}=2 \& J E R=8024$. [Accessed March 2015].

[88] National Institute for Statistics and Informatics (INEI) of Peru, "Households Access to Internet Service by Geographical Location 2001-2014," National Institute for Statistics and Informatics (INEI) of Peru, [Online]. Available: http://www.inei.gob.pe/estadisticas/indice-tematico/tecnologias-de-lainformacion-y-telecomunicaciones/. [Accessed June 2015].

[89] Telefonica del Peru (Movistar) \& Claro, "Monthly Internet Service Prices from Telefonica and Claro from Commercial Websites," Telefonica del Peru (Movistar) \& Claro. [Online].

[90] National Institute for Statistics and Informatics (INEI) of Peru, "Average Monthly Income from Work by Geographical Location 2004-2014," National Institute for Statistics and Informatics (INEI) of Peru, [Online]. Available: http://www.inei.gob.pe/estadisticas/indice-tematico/ocupacion-y-vivienda/.

[91] Supervisory Agency of Private Investment in Telecommunications (OSIPTEL) of Peru, "Statistic Indicators - Fixed Internet Service 1999-2009 \& 2010-2014," Supervisory Agency of Private Investment in Telecommunications (OSIPTEL) of Peru, [Online]. Available:

http://www.osiptel.gob.pe/documentos/indicadores-estadisticos. [Accessed May 2016].

[92] National Institute for Statistics and Informatics (INEI) of Peru, "National Population and Household Census 1993-2005-2007," National Institute for Statistics and Informatics (INEI) of Peru, [Online]. Available: https://www.inei.gob.pe/estadisticas/censos/. [Accessed May 2016].

[93] G. Q. Leon, "M.S. Thesis: Is it suitable to apply traditional propagation models and interference modeling methods for spectrum policy-making activities regarding small cell network architectures?," University of Colorado, Boulder, 2013.

[94] EDX Wireless, "EDX Wireless Software Appendices - Appendix A: 
Propagation Models," EDX Wireless, 2015.

[95] Jet Propulsion Laboratory-California Institute of Technology, "ASTER Advanced Spaceborne Thermal Emission and Reflection Radiometer," Jet Propulsion Laboratory-California Institute of Technology, [Online]. Available: http://asterweb.jpl.nasa.gov/GDEM.ASP. [Accessed November 2014].

[96] EDX Wireless, "Technology White Paper - The Application of Land Use/Land Cover (Clutter) Data to Wireless Communication System Design," EDX Wireless, 2008.

[97] N. Nayan, R. Zhao, N. Zhelev and W. Knospe, "Techno-economic analysis for rural broadband access networks," in The Eight Advanced International Conference on Telecommunications, Stuttgart, Germany, 2012.

[98] EDX Wireless, "EDX Software Reference Manual Appendices," EDX Wireless, 2012.

[99] J. Holmes and M. O'Rourke, "ITU Asia Pacific Centres of Excellence Expert Level Training on Telecom Network Cost Modelling for the Pacific," International Telecommunications Union, Apia, Samoa, 2012.

[100] Lumina Decision Systems, "What is Analytica?," Lumina Decision Systems, [Online]. Available: http://www.lumina.com/why-analytica/what-isanalytica1/.

[101] H. Gates, Research Meetings on Using Unmanned Aerial Vehicles to Provide Broadband Services, Boulder, Colorado, 2016.

[102] N. Lavars, "Google's Project Loon balloons to cover Sri Lanka with internet access," New Atlas, 30 July 2015. [Online]. Available: http://newatlas.com/google-project-loon-sri-lanka-universal-internetaccess/38690/. [Accessed October 2015].

[103] V. Woollaston, "Project Loon gets ready for global lift off: Google plans to have a 'ring' of internet balloons encircling the Earth by next year," Dailymail.com, 29 October 2015. [Online]. Available: http://www.dailymail.co.uk/sciencetech/article-3295055/Project-Loon-getsready-global-lift-Google-plans-ring-internet-balloons-encircling-Earthyear.html. [Accessed October 2015].

[104] Agency for Private Promotion Investment (ProInversion) of Peru, "1710$1770 \mathrm{MHz}$ and $2110-2170 \mathrm{MHz}$ bands (Blocks A and B) Nationwide - Auction Documents," Agency for Private Promotion Investment (ProInversion) of Peru, 2011. [Online]. [Accessed March 2014].

[105] Telefonica del Peru, "Press Release: Movistar Offers 4G LTE to Prepaid Customers," Telefonica del Peru, 12 February 2015. [Online]. Available: 
http://www.telefonica.com.pe/saladeprensa/noticias/2015/12022015.shtml. [Accessed March 2015].

[106] Telefonica del Peru, "Press Release: Telefonica Launchs 4G+ Technology that Allows Access to Fastest Internet Speed," Telefonica del Peru, 25 July 2016. [Online]. Available: http://www.telefonica.com.pe/saladeprensa/noticias/2016/25072016-full.shtml. [Accessed July 2016].

[107] Claro, "Press Release: Claro surpassed 600,000 4G LTE subscribers in Peru," Claro, 13 January 2015. [Online]. Available: http://www.claro.com.pe/portal/recursos/pe/pdf/600k-lineas-4G-LTE-Claro140115.pdf. [Accessed March 2016].

[108] Claro, "Claro 4G Coverage," Claro, [Online]. Available: http://cobertura.claro.com.pe/cobertura-movil-4g.php.

[109] OSIPTEL, "RESOLUCIÓN DE CONSEJO DIRECTIVO Nº 123-2014CD/OSIPTEL," 10 October 2014. [Online]. Available: https://www.osiptel.gob.pe/Archivos/ResolucionAltaDireccion/ConsejoDirectivo /Res123-2014-CD.pdf. [Accessed May 2015].

[110] B. Jones, "Google's Project Loon runs test of balloon-based internet service over Peru," Digital Trends, 30 September 2016. [Online]. Available: http://www.digitaltrends.com/mobile/google-project-loon-successful-test-peru/. [Accessed October 2016].

[111] B. Ortiz Bisso, "Google Tested Balloons in Peru," El Comercio, 2 October 2016. [Online]. Available: http://elcomercio.pe/tecnologia/actualidad/google-pusoprueba-sus-globos-peru-noticia1935725?ref=flujo_tags_539126\&ft=nota_1\&e=titulo. [Accessed October 2016].

[112] L. Rap, "Google's Internet Balloon Spotted Over Yellowstone," Fortune, 30 September 2016. [Online]. Available: http://fortune.com/2016/09/30/projectloon-yellowstone/. [Accessed October 2016].

[113] Ministry of Transportation and Communications (MTC) of Peru, "Technical Regulations - Peruvian Aeronautic Regulations - RAP 101 - Moored Balloons, Kites, Amateur Rockets and Unmanned Free Balloons," Ministry of Transportation and Communications (MTC) of Per, [Online]. Available: https://www.mtc.gob.pe/transportes/aeronautica_civil/normas/normas.html. [Accessed September 2016].

[114] Ministry of Transportation and Communications (MTC) of Peru, "Technical Regulations - Peruvian Aeronautic Regulations - RAP 101 - Part D Unmanned Free Balloons," Ministry of Transportation and Communications (MTC) of Peru, [Online]. Available: https://www.mtc.gob.pe/transportes/aeronautica_civil/normas/documentos/rap/ 
rap_rev15/rap101/rap_101_subparte_d_rev15.PDF. [Accessed October 2016].

[115] General Civil Aeronautic Directorate of Mexico, "Air Space Regulations for Aircraft Operation," General Civil Aeronautic Directorate of Mexico, [Online]. Available: http://www.ordenjuridico.gob.mx/Publicaciones/CDs2011/CDAereo/pdf/COF48. pdf. [Accessed October 2016].

[116] National Civil Aviation Administration of Argentina, "Aviation Regulations for Aircraft Operations," National Civil Aviation Administration of Argentina, [Online]. Available: http://www.anac.gov.ar/anac/web/uploads/normativa/resoluciones/resolucion828.pdf.

[117] Civil Aviation General Directorate of Ecuador, "Technical Regulations Aeronautic Regulations - RDAC 101 - Moored Balloons, Kites, Amateur Rockets and Unmanned Free Balloons," Civil Aviation General Directorate of Ecuador, [Online]. Available: http://www.aviacioncivil.gob.ec/wpcontent/uploads/downloads/2013/10/12.-RDAC-Parte-101.pdf. [Accessed October 2016].

[118] Federal Aviation Administration (FAA), "14 CFR Part 101-Moored balloons, kites, amateur rockets and unmanned free balloons," Federal Aviation Administration (FAA), [Online]. Available: https://www.law.cornell.edu/cfr/text/14/part-101. [Accessed October 2016].

[119] Flightradar24 - Live Air Traffic, "Live Air Traffic," Flightradar24 - Live Air Traffic, [Online]. Available: https://www.flightradar24.com/-10.86,-74.88/6. [Accessed March-September 2016].

[120] R. Struzak, "Intercomms-Mobile Telecommunications Via Stratosphere," 2003. [Online]. Available: http://www.intercomms.net/AUG03/content/struzak1.php. [Accessed 10 January 2016].

[121] A. Mohammed, A. Mehmood, F.-N. Pavlidou and M. Mohorcic, "The Role of High-Altitude Platforms (HAPs) in the Global Wireless Connectivity," in IEEE, 2011.

[122] T. C. Tozer and D. Grace, "High-altitude platforms for wireless communications," Electronics \& communication engineering journal, pp. 127-137, June 2001.

[123] HAPCOS Project (formerly CAPANINA Consortium), "COST297 High Altitude Platform for Communications and Other Services," HAPCOS Project (formerly CAPANINA Consortium), [Online]. Available: http://www.capanina.org. 
[124] A. Aragon-Zavala, J. L. Cuevas-Ruiz and J. A. Delgado-Penin, High-Altitude Platforms for Wireless Communications, West Sussex, United Kingdom: John Wiley \& Sons, Ltd., 2008.

[125] I. T. U.-R. Regulations, "Articles 1.66A, 4.23, 5.52A," TBD. [Online]. Available: http://www.itu.int/dms_pub/itu-s/oth/02/02/S02020000244501PDFE.PDF.

[126] NASA, "Earth Observatory - Glossary - Stratosphere," NASA - Earth Observatory, [Online]. Available:

http://earthobservatory.nasa.gov/Glossary/index.php?mode=alpha\&seg=s. [Accessed May 2015].

[127] Keck Institute for Space Studies, "AIRSHIPS: A New Horizon for Science," Keck Institute for Space Studies-California Institute of Technology-Jet Propulsion Laboratory, Pasadena, CA, 2013.

[128] E. H. Teets Jr., C. J. Donohue and P. T. Wright, "Meteorological Support of the Helios World Record High Altitude Flight to 96,863 Feet," National Aeronautics and Space Administration - Dryden Flight Research Center, Edwards, California, 2002.

[129] The Verge and B. Popper, "Google's balloons versus Facebook's drones: the dogfight to send internet from the sky - The race is on to own the stratosphere," The Verge, 7 March 2014. [Online]. Available: http://www.theverge.com/2014/3/7/5473692/facebook-drone-titan-aerospaceproject-loon. [Accessed June 2015].

[130] Raven Aerostar, "Raven Aerostar," Raven Aerostar, [Online]. Available: http://ravenaerostar.com/. [Accessed May 2014].

[131] Glaser, April; Townsend, Tess; Recode, "Recode - A breakthrough in Alphabet's balloon-based internet project means it might actually work," 16 February 2017. [Online]. Available: https://www.recode.net/2017/2/16/14640470/alphabets-balloon-based-internetloon-might-actually-work-algorithm. [Accessed February 2017].

[132] Metz, Cade; Wired, "Wired - Machine Learning Invades the Real World on Internet Balloons," 17 February 2017. [Online]. Available: https://www.wired.com/2017/02/machine-learning-drifting-real-world-internetballoons/. [Accessed February 2017].

[133] Ghoshal, Abhimanyu; The Next Web, "TNW - Google's balloon-powered internet service is 100x closer to becoming a reality," 17 February 2017. [Online]. Available: https://thenextweb.com/google/2017/02/17/googles-balloonpowered-internet-service-is-100x-closer-to-becoming-areality/\#.tnw_RGeS5uxl. [Accessed February 2017].

[134] Cisco, Design Guide - 802.11n Design and Deployment Guidelines, San Jose, 
CA: Cisco, 2008.

[135] Juniper Networks, White Paper - Coverage or Capacity - Making thee Best Use of 802.11n, Sunnyvale: Juniper Networks, Inc., 2011.

[136] D. D. Coleman and D. A. Westcott, CWNA: Certified Wireless Network Administrator Official Study Guide: Exam CWNA-106, John Wiley \& Sons, 2014, 2014.

[137] AirMagnet, White Paper-Impact of Legacy Devices on 802.11n Networks, AirMagnet, 2008.

[138] A. Ghosh, D. R. Wolter, J. G. Andrews and C. Runhua, "Broadband Wireless Access with WiMAX 802.16: Current Performance Benchmarks and Future Potential," IEEE Communications Magazine, vol. 0163, no. 6804, pp. 129-136, 2005.

[139] Carlson Wireless Technologies, Inc., RuralConnect TV White Space Radio, Arcata, CA: Carlson Wireless Technologies, Inc., 2015.

[140] WiMAX Forum, "WiMAX Deployment Considerations for Fixed Wireless Access in the 2.5 GHz and 3.5 GHz Licensed Bands," June 2005. [Online]. Available: http://www.wimaxforum.org/. [Accessed May 2015].

[141] S. Abdul Basit, Masters Thesis: Dimensioning of LTE Network - Description of Models and Tool, Coverage and Capacity Estimation of 3GPP Long Term Evolution Radio Interface, Helsinki University of Technology, 2009.

[142] Cisco, Release Notes for Cisco Wireless Controllers and Lightweight Access Points for Cisco Wireless Release 8.1.122.0, Cisco Systems, 2016.

[143] Joint Universities Computer Centre, "WLAN Scalability Test Report," Aruba Networks, [Online]. Available: http://www.arubanetworks.com/pdf/technology/whitepapers/wp_HiEd_JUCC_ Rpt.pdf. [Accessed December 2015].

[144] Voice + Data Networks, "Meru Networks' Airtime Fairness," Voice + Data Networks, [Online]. Available: http://www2.voicedata.com/SOLUTIONS/Meru_Networks_Airtime_Fairness.

[145] Supervisory Agency of Private Investment in Telecommunications (OSIPTEL), "The OSIPTEL in the deployment of the broadband and the social inclusion policy - Luis Pacheco Presentation," [Online]. Available: http://www.osiptel.gob.pe/websiteajax/Archivos/TwitterOsiptel/presentaciones/ sem-

asesores/4El_Osiptel_en_la_implementaci\%C3\%B3n_de_la_banda_ancha_y_s u_importancia_LuisPacheco.pd. [Accessed March 2014]. 
[146] P. P.-P. I. P. Agency, "Projects Portfolio-Telecommunications-Assigned Projects," [Online]. Available: http://www.proyectosapp.pe/modulos/JER/PlantillaProyectosResumenes.aspx? are $=0 \&$ prf $=2 \&$ jer $=5429 \&$ sec $=24$. [Accessed 2 February 2016].

[147] F. (. F. i. Telecommunications), "FITEL Yearly Report on Projects/programs 2015," 2015. [Online]. Available: http://www.fitel.gob.pe/pg/plan-anualproyectos-programas.php. [Accessed 15 February 2016].

[148] F. (. F. i. Telecommunications), "FITEL-Proposed Projects 2015," 2015. [Online]. Available: http://www.fitel.gob.pe/pg/proyectos-formulacion.php. [Accessed 15 February 2016].

[149] T. Schwengler, "8.4.2 LTE Throughput," in Wireless \& Cellular Communications - Class Notes for TLEN-5510, 2014, pp. 210--212.

[150] T. Schwengler, Wireless \& Cellular Communications - Class Notes for TLEN$5510,2014$.

[151] G. Leon, Is it suitable to apply traditional propagation models and interference modeling methods for spectrum policy-making activities regarding small cell network architectures?, University of Colorado at Boulder, 2013.

[152] NASA, "ASTER Global Digital Elevation Map," [Online]. Available: asterweb.jpl.nasa.gov. [Accessed 5 November 2014]. 


\section{Appendix 1: FITEL Projects and Bids for Broadband Deployments}

The main goal of the FITEL projects is to provide broadband Internet and Intranet access to public institutions, business and residential customers in the selected states. The following are current FITEL projects and status [18] [19]:

\begin{tabular}{|l|c|c|c|c|c|c|c|}
\hline \multicolumn{1}{|c|}{ Project Title } & $\begin{array}{c}\# \\
\text { Towns }\end{array}$ & $\begin{array}{c}\# \\
\text { Gov. } \\
\text { Inst. }\end{array}$ & Pop. & $\begin{array}{c}\text { Bid } \\
\text { Win- } \\
\text { ner }\end{array}$ & $\begin{array}{c}\text { Bid } \\
\text { Grant } \\
\text { (USD) }\end{array}$ & $\begin{array}{c}\text { Bid } \\
\text { Grant } \\
\text { Date }\end{array}$ & $\begin{array}{c}\text { Current } \\
\text { Stage }\end{array}$ \\
\hline $\begin{array}{l}\text { Installation of Broadband } \\
\text { Services for Integral Connectivity } \\
\text { and Social Development for the } \\
\text { State of Huancavelica }\end{array}$ & 308 & 1007 & $143 \mathrm{~K}$ & Gilat & $49.4 \mathrm{M}$ & $\begin{array}{c}\text { March } \\
2015\end{array}$ & $\begin{array}{c}\text { Deployme } \\
\text { nt }\end{array}$ \\
\hline $\begin{array}{l}\text { Installation of Broadband } \\
\text { Services for Integral Connectivity } \\
\text { and Social Development for the } \\
\text { State of Ayacucho }\end{array}$ & 304 & 901 & $179 \mathrm{~K}$ & Gilat & $55.3 \mathrm{M}$ & $\begin{array}{c}\text { March } \\
2015\end{array}$ & $\begin{array}{c}\text { Deployme } \\
\text { nt }\end{array}$ \\
\hline $\begin{array}{l}\text { Installation of Broadband } \\
\text { Services for Integral Connectivity } \\
\text { and Social Development for the } \\
\text { State of Apurimac }\end{array}$ & 285 & 602 & $123 \mathrm{~K}$ & Gilat & $42.3 \mathrm{M}$ & $\begin{array}{c}\text { March } \\
2015\end{array}$ & $\begin{array}{c}\text { Deployme } \\
\text { nt }\end{array}$ \\
\hline $\begin{array}{l}\text { Broadband Integral Connectivity } \\
\text { for Social Development in } \\
\text { Northern Peru-State of } \\
\text { Lambayeque }\end{array}$ & 309 & 501 & $302 \mathrm{~K}$ & $\begin{array}{c}\text { Telefo } \\
\text { nica }\end{array}$ & $31.5 \mathrm{M}$ & $\begin{array}{c}\text { March } \\
2015\end{array}$ & $\begin{array}{c}\text { Deployme } \\
\text { nt }\end{array}$ \\
\hline $\begin{array}{l}\text { Installation of Broadband } \\
\text { Services for Integral Connectivity } \\
\text { and Social Development for the } \\
\text { States of Tumbes \& Piura }\end{array}$ & 463 & 717 & $336 \mathrm{~K}$ & $\begin{array}{c}\text { Redes } \\
\text { Andin } \\
\text { as }\end{array}$ & $98.5 \mathrm{M}$ & $\begin{array}{c}\text { Dec } \\
2015\end{array}$ & $\begin{array}{c}\text { Deployme } \\
\text { nt }\end{array}$ \\
\hline $\begin{array}{l}\text { Installation of Broadband } \\
\text { Services for Integral Connectivity } \\
\text { and Social Development for the } \\
\text { State of Cajamarca }\end{array}$ & 927 & 1,432 & $356 \mathrm{~K}$ & $\begin{array}{c}\text { Redes } \\
\text { Andin } \\
\text { as }\end{array}$ & $149.6 \mathrm{M}$ & $\begin{array}{c}\text { Dec } \\
2015\end{array}$ & $\begin{array}{c}\text { Pre- } \\
\text { Deployme } \\
\text { nt }\end{array}$ \\
\hline $\begin{array}{l}\text { Installation of Broadband } \\
\text { Services for Integral Connectivity } \\
\text { and Social Development for the } \\
\text { State of Cusco }\end{array}$ & 424 & 581 & $177 \mathrm{~K}$ & Gilat & $108.4 \mathrm{M}$ & $\begin{array}{c}\text { Dec } \\
2015\end{array}$ & $\begin{array}{c}\text { Pre- } \\
\text { Deployme } \\
\text { nt }\end{array}$ \\
\hline $\begin{array}{l}\text { Installation of Broadband } \\
\text { Services for Integral Connectivity } \\
\text { and Social Development for the } \\
\text { State of Ica }\end{array}$ & 85 & $75 \mathrm{~K}$ & - & $28.1 \mathrm{M}$ & $\begin{array}{c}\text { Approved } \\
\text { Viability } \\
\text { (March } \\
2015)\end{array}$ \\
\hline
\end{tabular}




\begin{tabular}{|c|c|c|c|c|c|c|}
\hline $\begin{array}{l}\text { Installation of Broadband } \\
\text { Services for Integral Connectivity } \\
\text { and Social Development for the } \\
\text { State of Lima }\end{array}$ & 264 & $193 \mathrm{~K}$ & - & $69.9 \mathrm{M}$ & - & $\begin{array}{c}\text { Approved } \\
\text { Viability } \\
\text { (March } \\
2015) \\
\end{array}$ \\
\hline $\begin{array}{l}\text { Installation of Broadband } \\
\text { Services for Integral Connectivity } \\
\text { and Social Development for the } \\
\text { State of Tacna }\end{array}$ & 51 & $39 \mathrm{~K}$ & - & $17 \mathrm{M}$ & - & $\begin{array}{c}\text { Approved } \\
\text { Viability } \\
\text { (April } \\
2015) \\
\end{array}$ \\
\hline $\begin{array}{l}\text { Installation of Broadband } \\
\text { Services for Integral Connectivity } \\
\text { and Social Development for the } \\
\text { State of Moquegua }\end{array}$ & 56 & $47 \mathrm{~K}$ & - & $18.3 \mathrm{M}$ & - & $\begin{array}{c}\text { Approved } \\
\text { Viability } \\
\text { (June } \\
2015 \text { ) }\end{array}$ \\
\hline $\begin{array}{l}\text { Installation of Broadband } \\
\text { Services for Integral Connectivity } \\
\text { and Social Development for the } \\
\text { State of Amazonas }\end{array}$ & 247 & $127 \mathrm{~K}$ & - & $63 \mathrm{M}$ & - & $\begin{array}{c}\text { Approved } \\
\text { Viability } \\
\text { (July } \\
2015 \text { ) } \\
\end{array}$ \\
\hline $\begin{array}{l}\text { Installation of Broadband } \\
\text { Services for Integral Connectivity } \\
\text { and Social Development for the } \\
\text { State of Ancash }\end{array}$ & 429 & $192 \mathrm{~K}$ & - & $93 \mathrm{M}$ & - & $\begin{array}{c}\text { Proposal } \\
\text { Review } \\
\text { (April } \\
2015 \text { ) } \\
\end{array}$ \\
\hline $\begin{array}{l}\text { Installation of Broadband } \\
\text { Services for Integral Connectivity } \\
\text { and Social Development for the } \\
\text { State of Junin }\end{array}$ & 324 & $285 \mathrm{~K}$ & - & $74.9 \mathrm{M}$ & - & $\begin{array}{c}\text { Proposal } \\
\text { Review } \\
\text { (April } \\
2015)\end{array}$ \\
\hline $\begin{array}{l}\text { Installation of Broadband } \\
\text { Services for Integral Connectivity } \\
\text { and Social Development for the } \\
\text { State of Puni }\end{array}$ & 418 & $269 \mathrm{~K}$ & - & $87.9 \mathrm{M}$ & - & $\begin{array}{c}\text { Proposal } \\
\text { Review } \\
\text { (April } \\
2015) \\
\end{array}$ \\
\hline $\begin{array}{l}\text { Installation of Broadband } \\
\text { Services for Integral Connectivity } \\
\text { and Social Development for the } \\
\text { State of Arequipa }\end{array}$ & 162 & $122 \mathrm{~K}$ & - & $63.9 \mathrm{M}$ & - & $\begin{array}{c}\text { Project } \\
\text { Proposal } \\
(4 \mathrm{Q} 2015)\end{array}$ \\
\hline $\begin{array}{l}\text { Installation of Broadband } \\
\text { Services for Integral Connectivity } \\
\text { and Social Development for the } \\
\text { State of Huanuco }\end{array}$ & 258 & $139 \mathrm{~K}$ & - & $48.4 \mathrm{M}$ & - & $\begin{array}{c}\text { Project } \\
\text { Proposal } \\
(4 \mathrm{Q} 2015)\end{array}$ \\
\hline $\begin{array}{l}\text { Installation of Broadband } \\
\text { Services for Integral Connectivity } \\
\text { and Social Development for the } \\
\text { State of La Libertad }\end{array}$ & 447 & $425 \mathrm{~K}$ & - & $65.5 \mathrm{M}$ & - & $\begin{array}{c}\text { Project } \\
\text { Proposal } \\
(4 \mathrm{Q} 2015)\end{array}$ \\
\hline $\begin{array}{l}\text { Installation of Broadband } \\
\text { Services for Integral Connectivity } \\
\text { and Social Development for the } \\
\text { State of Pasco }\end{array}$ & 108 & $76 \mathrm{~K}$ & - & $25 \mathrm{M}$ & - & $\begin{array}{c}\text { Project } \\
\text { Proposal } \\
(4 \mathrm{Q} 2015)\end{array}$ \\
\hline $\begin{array}{l}\text { Installation of Broadband } \\
\text { Services for Integral Connectivity } \\
\text { and Social Development for the } \\
\text { State of San Martin }\end{array}$ & 230 & $166 \mathrm{~K}$ & - & $48 \mathrm{M}$ & - & $\begin{array}{c}\text { Project } \\
\text { Proposal } \\
(4 \mathrm{Q} 2015)\end{array}$ \\
\hline
\end{tabular}


For the granted and coming FITEL projects, ProInversion and FITEL set the following contract statements and access network specifications for the broadband projects:

- The operator will transfer the ownership of the access network to FITEL at the end of the finance contract.

- The access network elements consist of the metallic structures, towers (selfsupported), foundations and land, and all passive element part of the access network, and will become property of the Peruvian Government after signing the proper paperwork. The active equipment will remain property of the operator.

- The investment period of the access network has a maximum duration of 12 months from the closing date, which consists of the activities defined in the installation and supervision periods stated in the technical specifications of the access network. This period ends with the acceptance document of installation and service testing of the access network.

- The operation period is 120 months from the day after the ending of the investment period. The operator will operate and maintain the access network to ensure the network will continue working and provision of commercial services.

- The operator will have to provide Internet and Intranet access to the number of towns specified in the bid using a broadband terrestrial network. 
In the towns, the operator will have to provide services to public, private, residential and business customers.

- The operator will have to mandatorily provide services to the government institutions such as healthcare centers and posts, police stations and schools.

- The installation is free for mandatory and other customers, as long as the total number of customer premise equipment (CPE) is not greater than 4,000 .

- The Internet service monthly rates will be the following for government institutions:

\begin{tabular}{|c|c|c|}
\hline Download Speed & $\begin{array}{c}\text { Guaranteed } \\
\text { Minimal Speed (\%) }\end{array}$ & $\begin{array}{c}\text { Monthly Rate with } \\
\text { Taxes (S/.) }\end{array}$ \\
\hline $2 \mathrm{Mbps}$ & $40 \%$ & 97,59 \\
\hline $4 \mathrm{Mbps}$ & $40 \%$ & 142,06 \\
\hline
\end{tabular}

- The mandatory customers will purchase a minimum of $2048 \mathrm{kbps}$ in downlink and 512kbps uplink and 40\% guaranteed speed.

- Carry out interconnection agreements so customers can communicate with users in other networks and vice versa.

- Access network equipment and CPEs must be new.

- The access network equipment must comply with the ITU-R and ITU-T technical recommendations.

- Use frequency bands that the operator (contractor) is sure that there are channels or frequencies available in a period no less than six months after 
the closing date. The operator must check with the General Directorate of Communications of the Ministry of Transportation and Communications.

- The network must be able to handle the future demand in the selected towns for the provided services.

- The access network must consist of the following nodes:

- District Node: Interconnects with the transport network and can have point of presence (POP).

- Intermediate Node: Retransmit signal from a District Node to a terminal Node, and can have a POP.

○ Terminal Node: Operates as a POP.

- In case that selected towns due to their proximity to each other can be served by only one node (district or terminal node), the operator can use this exception, as far as the towns are not farther than a 2000 meter distance.

- The operator must install, operate and maintain an energy system in selected towns where there is not permanent public electric grid supply. This energy system will be included as part of the access network.

- District nodes of the access network must have at least one diesel power generator, rectifier charger, control/transfer board, peak voltage breaker and batteries.

- Intermediate and terminal nodes of the access network must have at least a rectifier charger, control/transfer board, peak voltage breaker and batteries. 
- Promptly replace at the expense of the operator equipment and infrastructure (from the access network to the CPE) as many times as needed.

- The availability of the access network is $98 \%$ per year.

- These requirements include the following appendices:

○ List of selected towns and mandatory government customers.

- List of additional selected towns.

- Technical features of the access module (government customer module).

- Technical requirements for metal infrastructure and foundation of self-supported towers.

In this dissertation, the following FITE bids' technical requirements were excluded from the wireless access network design:

- Computer stations for schools, health posts, etc.

- Intranet service and content, social awareness campaign, training.

- Installation and operation of a NOC.

- Customer service center and operation and maintenance centers. 


\section{Appendix 2: High Altitude Platforms (HAPs) for Providing Broadband Services}

\section{A2.1 Background}

A High Altitude Platform (HAP) station is defined by the International Telecommunications Union (ITU) as "a station located on an object at an altitude of $20 \mathrm{~km}$ to $50 \mathrm{~km}$ and at a specified, nominal, fixed point relative to the Earth" [120] In practical deployments, HAPs are quasi-stationary aerial platforms located at a height of $17-22 \mathrm{~km}$ above Earth's surface in the stratospheric region of the atmosphere [121], although in recent years, Project Loon proposed and tested nonstationary HAPs traveling in the stratosphere. HAP stations are also known as High Altitude Long Endurance (HALE) station, Stratospheric Platform Radio (SPR) or Stratospheric Satellites. HAPs may operate in the stratosphere for purposes of research (i.e. remote sensing and environmental monitoring), military applications, communications (broadband, cellular, broadcast or emergency communications), among other commercial services such as navigation and position location systems, surveillance, traffic monitoring.

The platforms may be aerostatic (balloons and airships) or aerodynamics (aircrafts) which can have a manned or unmanned operation with remote control from the ground [121] [122]. Aerostatic platforms use a lifting lighter-than-air gas (i.e. diatomic hydrogen or helium) in an envelope to provide buoyancy. Balloons regained attention in the 1990s due to technology developments such as new 
plastic envelope materials more resistant to gas pressure, UV solar radiation and leak proof to lighter-than-air gases. Airships use very large semi-rigid or non-rigid gas containers which can have a length of $100 \mathrm{~m}$ or more. The large size of airships is an advantage due to the ability to accommodate larger and heavier payloads and solar energy systems, but it is also a drawback due to the increased dynamic drag during takeoff, fly and landing. Aerodynamic platforms exploit the aerodynamic lift and need to move forward to fly in the air. Aircrafts designed to fly in the stratosphere, under low air density conditions, require using components lighter than commercial aircrafts components and large wingspan to stay aloft.

Major challenges for HAPs are station keeping and need for refueling. For balloons or airships, electric motors and propellers are used to keep a relatively fixed position flying against stratospheric winds. For aircrafts, station keeping is achieved by also flying against the wind but in a roughly circular tight path.

Refueling is achieved in most cases by using solar energy systems (solar cells and regenerative fuel cells) to power the motors and payload [122].

\section{A2.1.1 HAPs for Communications}

HAPs can be deployed to provide broadcast/multicast HDTV signal, 4G and beyond 4G services, broadband fixed wireless access, backhaul links for groundbased pico-cells, emergency or disaster services, and military communications [121] [122]. These communication platforms come as an alternative to satellites (geostationary or low earth orbit) and terrestrial networks for delivering wireless broadband services at higher frequencies, which require line-of-sight conditions. 
HAPs have the capability to overcome major limitations of satellite and terrestrial networks by operating in a relatively fixed location in the stratosphere at $22 \mathrm{~km}$ above the ground carrying a communications payload. Communication services using geostationary (GEO) satellites present limitations such as high propagation losses (in the order of $200 \mathrm{~dB}$ ), size of both the on-board and customer antennas, in addition to the roundtrip propagation delay of around $250 \mathrm{~ms}$. In the case of low earth orbit (LEO) satellites, major limitation relies on the large number of satellites and associated cost required to provide continuous service, in addition to technical complexities for rapid hand over not only between cells in a single platform but also between platforms. In the case of terrestrial networks, providing communication services to wide areas or rural isolated areas requires large infrastructure deployments which may present high upfront and operating costs [122].

HAPs for communications exploit the best features of both terrestrial and satellite communications [121] [122]: 1) large coverage area in comparison to terrestrial systems (including reduced rain attenuation), 2) flexibility to respond to traffic demands (flexible and responsive frequency reuse patterns and cell sizes), 3) low cost in comparison to launching and operating GEO or LEO satellites, 4) incremental deployment (adding HAPs based on demand growth), 5) rapid deployment (in comparison to launching satellites and roll-out terrestrial networks), 6) platform and payload upgrading (HAPs can return to the ground for 
maintenance and upgrade), and 7) environmentally friendly (using solar energy and avoiding roll-out in natural landscapes to reach rural areas).

The coverage area set by HAPs is mostly determined by the line-of-sight propagation and angle of the ground equipment (alignment and aperture of the antenna). At altitude of $20 \mathrm{~km}$, HAPs can provide coverage up to $200 \mathrm{~km}$ radius, which can be subdivided into smaller coverage zones or cells. Using frequency reuse and adaptive resource allocation techniques can provide efficient use of bandwidth and maximize capacity. The communications payload to provide services consist of radio equipment to connect to 1 ) end user equipment (access links), 2) fiber backbone (backhaul links), and In the case of deploying a constellation of HAPs, additional inter-HAPs links need to be deployed [122].

An additional advantage of HAPs in comparison to satellites is that payloads can return to the ground for maintenance and upgrade.

\section{A2.1.2 HAP research and industry initiatives}

The following are major HAP research and industry initiatives in the early and mid-2000s [121] [123] [124]:

- CAPANINA and HAPCOS projects: The CAPANINA project was a European initiative aimed to explore the development of broadband communications capabilities from HAPs using airships. The HAPCOS (High Altitude Platform for Communications and Other Services) project or COST Action 297 was also an European initiative which main objective was to 
increase knowledge and understanding of the use of HAPs for delivery of communications and other services, by exploring, researching and developing new methods, analyses, techniques and strategies for developers, service providers, system integrators and regulators.

- SkyStation International: Initiative in the United States aimed to deploy geo-stationary solar-powered aerostatic high-altitude platform systems at altitudes of around $21 \mathrm{~km}$ in the lower stratosphere over major metropolitan areas using proprietary technologies.

- Skynet: Telecommunications initiative developed in Japan and led by the Yokosuka Communications research Laboratory to deploy airships to provide communication services in the $28 \mathrm{GHz}$ band and also broadcasting services to serve most of Japan.

- Helios, Pathfinder and Centurion: The programs were led by AeroVironment in the United States to deploy unmanned solar-powered aircrafts flying against the wind or in a circular tight path. These aircrafts demonstrated flight endurance at up to $25 \mathrm{~km}$.

- Heliplat: Part of the Helinet project to develop solar-powered crafts funded by the European Commission for broadband telecommunication services, environmental monitoring and vehicle localization.

- Halo project: Developed by Angel technologies uses the Proteus aircraft to operate at altitudes from 16 to $18 \mathrm{~km}$ to provide broadband communication services to an area up to $40 \mathrm{~km}$ in diameter. 
Other more current initiatives include the following [52] [60] [61] [62] [64] [65]:

- Project Loon (Google X): Delivering broadband services using superpressure balloons travelling in the stratosphere (20km above the ground) to connect customers in rural and remote areas to the Internet.

- Project SkyBender (Google X): Currently testing in New Mexico Titan's high-altitude-solar-powered drones using millimeter wave technology to transmit at data rates higher than $4 \mathrm{G}$ LTE.

- Ascenta (Facebook): Currently testing solar-powered drones using free space optics (high-powered laser beams) to achieve high data rate transmissions.

\section{A2.1.3 Telecommunications regulatory and policy environment for HAPs}

Regulation and policies for using HAPs for communication services is

expected to come from two areas: 1) spectrum and provision of communication services, and 2) aeronautical operation.

In the spectrum and provision of communication services, the ITU Radiocommunication Sector (ITU-R) defines the spectrum allocated for HAPs. The ITU-R limits the following bands for transmissions to or from HAP stations in Region 2 (the Americas) [125]:

- $1,885 \mathrm{MHz}-1,980 \mathrm{MHz}$ and $2110 \mathrm{MHz}-2160 \mathrm{MHz}$ bands to provide International Mobile Telecommunications (IMT) services. Any other stations 
are not precluded from using these bands and there is not any priority established in the regulations.

- $47.2 \mathrm{GHz}-47.5 \mathrm{GHz}$ and $47.9 \mathrm{GHz}-48.2 \mathrm{GHz}$ bands to provide fixed services. Currently Google and Facebook are testing providing broadband services using other bands and technologies such as unlicensed Wi-Fi, unlicensed and licensed millimeter-wave and optical frequencies (lasers). Since spectrum regulations ultimately depends on government regulators, it is assumed that these tech companies are discussing with regulators using these bands to provide broadband services in unserved and underserved rural areas.

\section{A2.2 Stratospheric Environment}

The stratosphere is the region of the atmosphere between the troposphere and mesosphere. The stratosphere begins at approximately $8 \mathrm{~km}(26 \mathrm{kft})$ at the poles and $15 \mathrm{~km}(49 \mathrm{kft})$ at the equator and extends to approximately $50 \mathrm{~km}(164 \mathrm{kft})$ [126]. In this atmospheric region, temperature increases with altitude due to absorption of solar radiation by ozone. The environment at stratospheric heights resembles more to outer space than sea level: low temperatures, low air pressure, higher solar irradiance, and lower convective heat transfer [127]. The temperature in the stratosphere can reach extreme cold temperatures as low as $-55^{\circ} \mathrm{C}\left(218^{\circ} \mathrm{K}\right.$ or $\left.-67^{\circ} \mathrm{F}\right)$. At night, exposed surface temperatures can drop as low as $-79^{\circ} \mathrm{C}$ due to radiation cooling. However, during the day, solar and UV radiation can be higher than at sea level. 


\section{Stratospheric Winds}

The winds in the stratosphere vary depending on altitude, latitude and time of year. Figure 60 shows the annual averaged wind speeds at different latitudes at $20 \mathrm{~km}(65,000 \mathrm{ft})$ from 1948 to 2002 . Our study areas in Peru are located in the southern hemisphere at latitudes between $12^{\circ}$ and $13^{\circ}$ south. From this figure, we estimate that for these latitudes the averaged wind speed ranges from $7 \mathrm{~m} / \mathrm{s}$ $(25.2 \mathrm{~km} / \mathrm{hr}$ or $15.6 \mathrm{mi} / \mathrm{hr})$ to $17 \mathrm{~m} / \mathrm{s}(61 \mathrm{~km} / \mathrm{h}$ or $38 \mathrm{mi} / \mathrm{hr})$. These wind speeds seem benign for stability and operation of HAPs or unmanned aerial vehicles (UAVs) at $20 \mathrm{~km}$ altitude in the lower stratosphere.

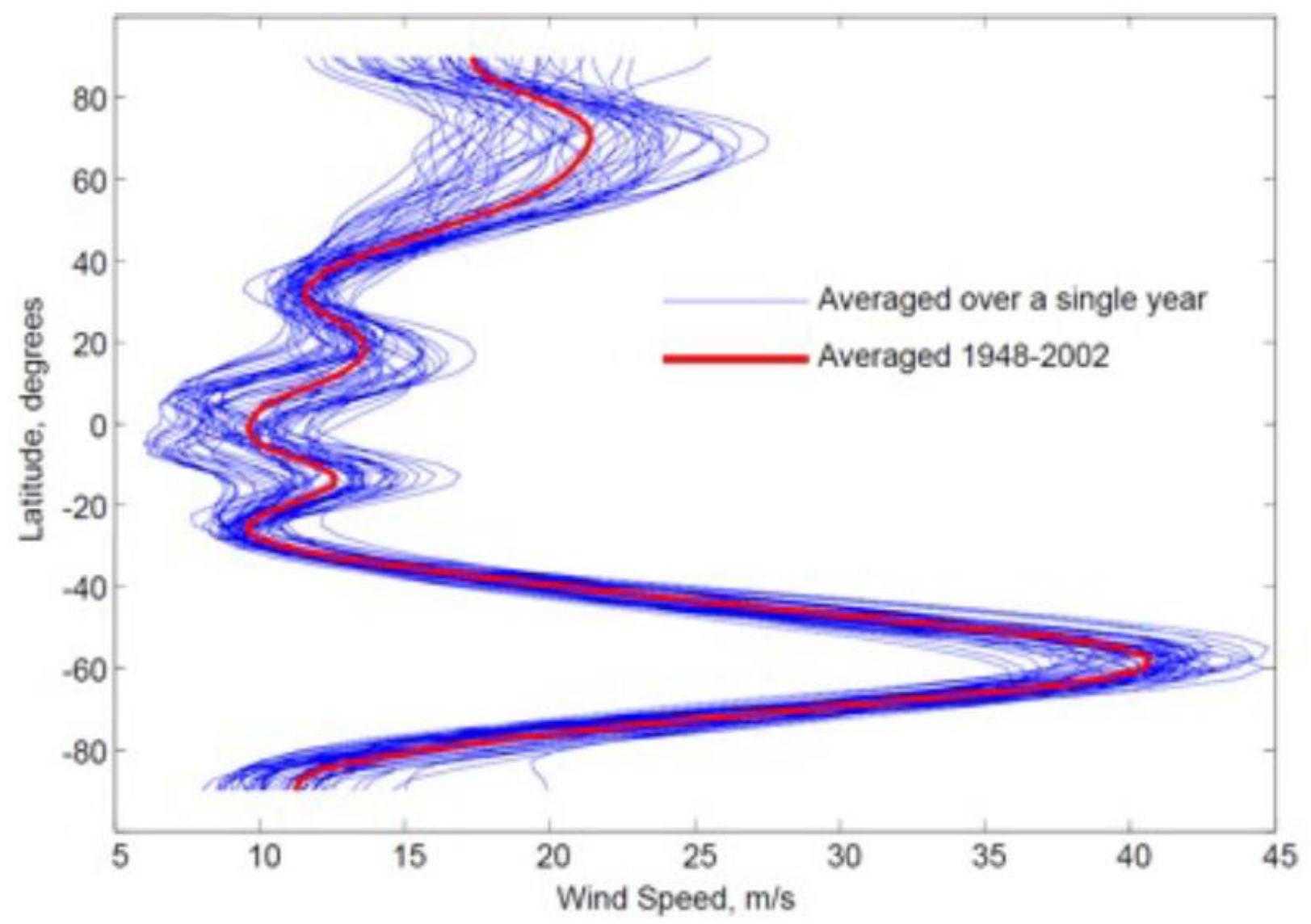

Figure 60. Annual averaged wind speeds at different latitudes from 1948 to 2002 at $20 \mathrm{~km}$ above the ground [127]. 
As an example of the steady behavior of winds in the lower stratosphere,

Figure 61 and Figure 62 show detailed wind speed and direction profiles from August 13, 2002 at the Pacific Missile Range Facility (PMRF) in Hawaii, located at latitude $22^{\circ}$ north. We can observe that wind speed and direction are consistent and steady during the day.

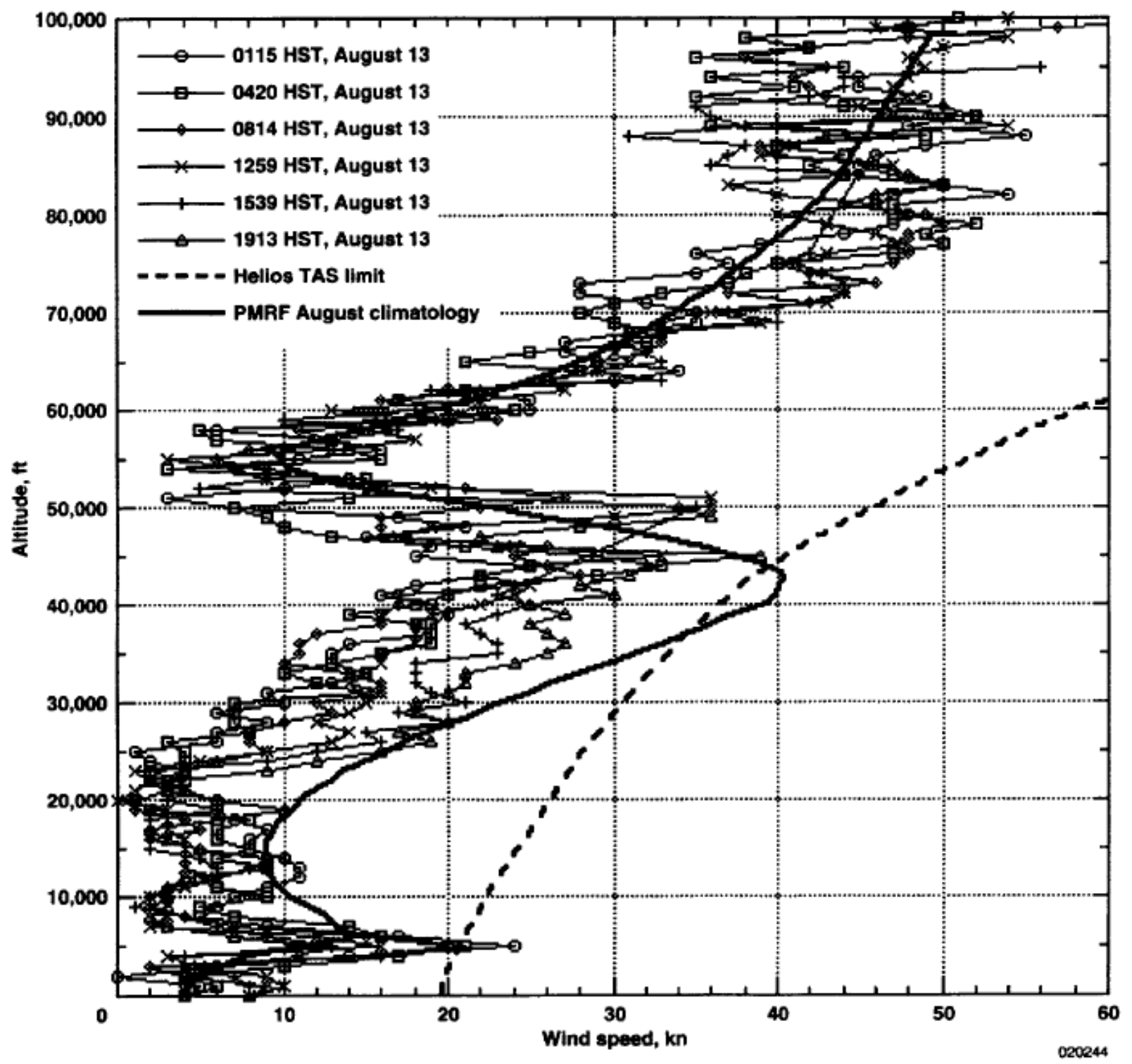

Figure 61. Wind speed profiles from August 13 with Helios TAS limit and PMRF August wind climatology. [128] 


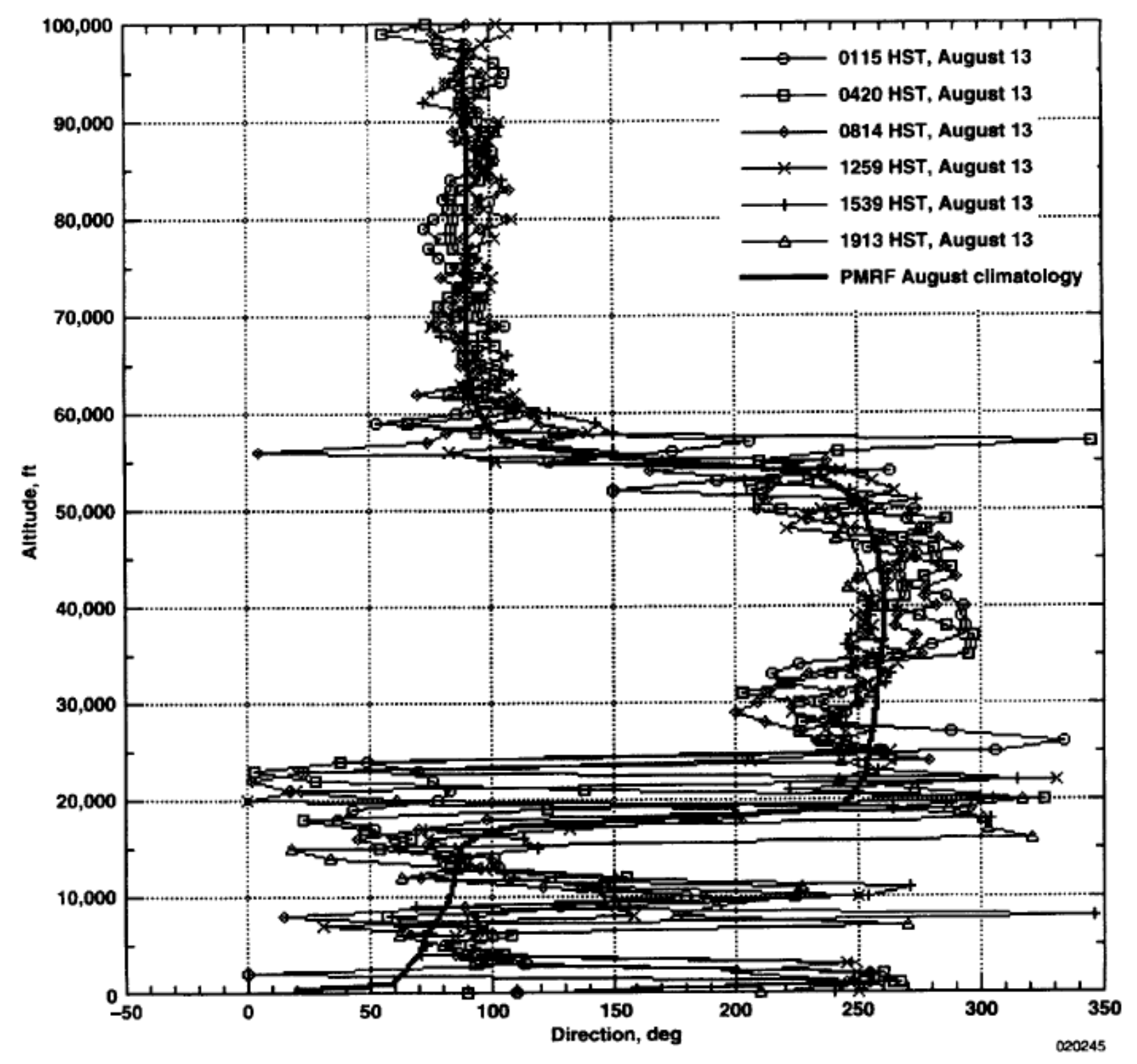

Figure 62. Wind direction profiles from August 13 with PMRF August wind direction climatology [127].

Based on these wind profiles, we assume that winds in the lower stratosphere are steady. We will use later these wind profiles for the flight analysis of the stratospheric balloons used in the Project Loon. 


\section{A2.3 Project Loon (Google X)}

The Project Loon was developed by Google X and officially launched in 2013. The project aims to deploy a network of balloons traveling in the lower stratosphere to connect people in rural and remote areas, help fill coverage gaps, and bring communications after disaster or emergency situations. It uses superpressure balloons which will float in the stratosphere at altitudes between $17 \mathrm{~km}$ $(55.7 \mathrm{kft})$ to $25 \mathrm{~km}(82 \mathrm{kft})$, twice as high as airplanes, $9 \mathrm{~km}(29.5 \mathrm{kft})$ to $12 \mathrm{~km}$ (39.3kft), and the weather. The balloons will move toward specific locations or areas varying speed and direction using the steady stratospheric winds and wind layers at different altitudes. For providing services, Loon project executives have announced partnerships with telecommunications companies to share spectrum to connect phones and other LTE-enabled devices directly to the balloon network [52] [54] [55]. Then communications are routed across the balloon network and sent back down to the Internet.

In the late 90s there were initiatives to use low earth orbit satellite to provide global cellphone coverage, for example the Iridium and Globalstar systems, however later the companies declared bankruptcy. One problem at that time was that end user would have required bulky custom handsets to receive the signal, which is not a current obstacle now due to the widespread proliferation of cheap powerful smartphones $u$ other customers premise equipment [129]. 


\section{A2.3.1 Aerial Vehicle and Payload}

The balloons network aims to deploy stratospheric nodes using unmanned aerial vehicles as high latitude towers. These vehicles consist of a balloon envelope connected to a payload. Based on a patent filed by Google titled Balloon Power Sources with a Buoyancy Trade-Off [53], this subsection describes the envelope and payload which carries the navigation, telecommunications and energy equipment.

The balloons used for this project are super-pressure balloons designed and manufactured by Raven Aerostar [130]. Super-pressure balloon envelopes are able to stay aloft at high altitudes and filled with lighter-than-air gas are pressurized during both day and night operations for long-duration missions, which provides greater stability at float altitude. Figure 63 shows the altitude path for supper pressure balloons. We can observe that cold temperatures at nigh have a minimal impact on the overall balloon buoyancy in comparison to zero pressure balloons. The balloon envelope can be made of materials such as of a polyethylene, highlyflexible latex, rubber, or metalized mylar. The envelope is filled with hydrogen, helium or any other lighter-than-air gas. The envelope connects to the payload using a cord which includes a cut-down system to allow separating the balloon from the payload. This system allows the reuse of parts of the payload by deploying a recovery parachute which will bring safely the payload down to the ground. 


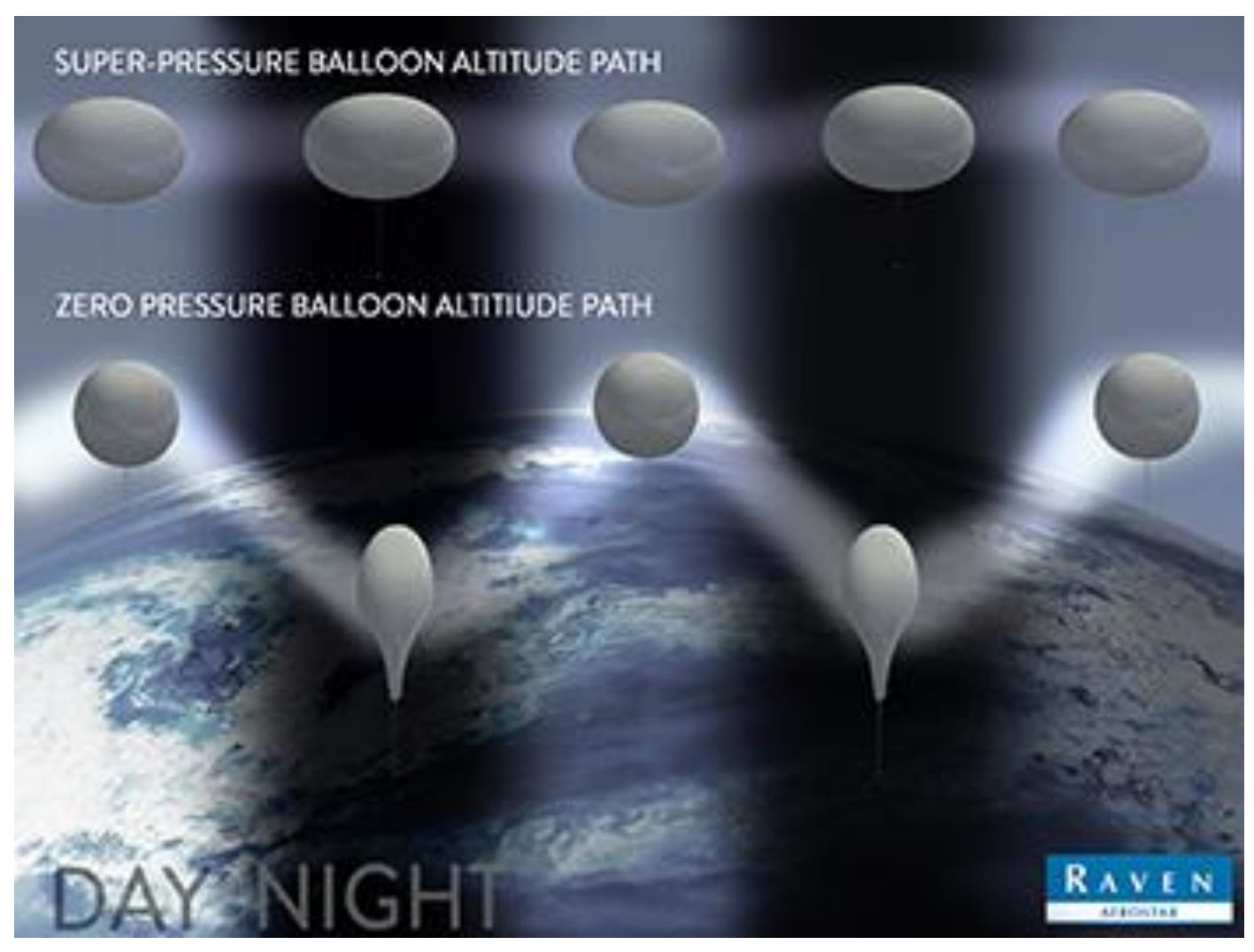

Figure 63. Super-pressure balloons altitude path at day and night [130].

The payload of the balloon consists of the following subsystems [53]:

- Computational subsystem: Processor and on-board data storage such as a memory.

- Energy subsystem: Solar panels and rechargeable battery.

- Navigation subsystem: Equipment to implement station-keeping functions to maintain position or move to a position in accordance to the desired network topology.

- Telecommunications subsystem: Include Free Space Optical (FSO) and Radio Frequency (RF) equipment for the backhaul and access radio links. 
- Sensing subsystem: It potentially includes video cameras, motion sensors, environmental data sensors, etc.

- Altitude control subsystem: Includes equipment and components to vary the buoyancy of the vehicle.

The following subsection describes the operation of the balloon using the listed subsystems.

\section{A2.3.2 Balloons Operation}

\section{Vehicle Navigation}

An important technological innovation of this project, in comparison to previous HAP initiatives, is using steady stratospheric winds as a source of propulsion for the balloons to move in a horizontal direction to a specific location or along a planned route. This allows reducing the payload weight and overall cost of each balloon since it will not require a propulsion subsystem (i.e. motors and energy sources). To move in a horizontal direction, the vehicle, first, determines a higher or lower altitude wind which corresponds to the given horizontal direction, and, second, increases or decreases the buoyancy of the envelope to reach such altitude.

\section{Telecommunication Services}

The main goal of Project Loon is to connect people in rural remote areas and this goal is achieved by the telecommunications subsystem placed in the payload. 
This subsystem runs using energy from the solar panels during the day and from the batteries during night. The following two types of communication technologies are used in the subsystem:

- Free-space optical (FSO) communications: It uses ultra-bright (highpower or high-output) LEDs or lasers for the optical transmission and photodiodes for the optical reception. FSO will enable links that may extend as far as 100 miles and be capable of achieving data rate speeds of 10 to 50Gbps to communicate balloons with each other as the backbone of the balloon network. Each balloon may include one or more FSO transmitters and receivers which may be implemented with mechanical systems to achieve high precision FSO alignment.

- Radio-frequency (RF) communications: It can use various different RF equipment (transceivers and antennas) and air-interface protocols which might include IEEE 802.11, GSM, CDMA, UMTS, EV-DO, WiMAX, LTE, or other proprietary protocols. Balloons may also use RF systems to communicate to other balloons. RF will enable links capable of achieving data rate speeds on the order of approximately $10 \mathrm{Mbps}$ as the access links to communicate balloons with ground stations and final users. The Project Loon conducted Internet service trials initially using Wi-Fi, but in 2014, LTE was used for the user devices. In 2015, Project Loon executives have announced partnerships with telecommunications companies to share 
cellular spectrum and licenses to connect phones and other LTE-enabled devices directly to the balloon network.

\section{A2.3.3 Balloons Network}

The Project Loons aims to deploy mesh networks for packet data communications formed by high-latitude balloons deployed in the stratosphere ( $17 \mathrm{~km}$ to $25 \mathrm{~km}$ above the ground). Each balloon is configured to change its horizontal position (latitudinal or longitudinally) by adjusting its vertical position.

The balloons network can be homogenous (similar configuration) or heterogeneous (different configuration) balloons. There are two types of balloon configurations for the network: 1) super-node, and 2) sub-node. A super-node balloon can be configured to communicate with nearby super-node balloons using FSO links and with sub-node balloons using RF links. Super-node balloons collectively will work as the backhaul of the balloon network. A sub-node balloon can be configured to communicate using RF links and will work to relay communications from the super-nodes to ground base stations as the access network. Additionally, both super-node and sub-node balloons can be configured as downlink balloons to provide high-capacity air-ground links (balloons to groundbased stations) via optical or RF links. A network can include multiple downlink balloons. In some particular cases, balloons could also establish communication links with space-based satellites.

The ground-based stations may include transceivers, transmitters, or receivers for communication via $\mathrm{RF}$ links or optical links with the balloon network. 
In certain settings, these stations may be configured as access points which user devices can connect to the balloon network. The ground-based stations may be also configured as gateways between the balloon network and more networks, the Internet, or a cellular service provider's network.

Project Loon conducted the first experimental pilot in New Zealand. In this pilot, the balloon was able to fly from New Zealand to Latin America and back delivering Internet connection. Other pilots were conducted in California's Central Valley and in Northeast Brazil. The results of the pilots are being used to improve the technology in preparation for the next stages of the project [52]. The project worked with telcos such as Vodafone in New Zealand, Telstra in Australia, and Telefonica, Vivo and Telebras in Latin America, and it's in the way of working deals with several new network operators around the world. A potential business model is to partner with these companies which already have LTE spectrum and have a telecom business (infrastructure, sales and billing departments, etc.) running, so Google obtains permission to use the spectrum and use the balloons as cellular towers in the sky. In this way, both telcos and Google can share the revenue for each new customer reached in rural areas [54]. Tests using LTE provided $2 \mathrm{MBs}$ to a ground antenna and $5 \mathrm{Mbps}$ to a handset [55].

\section{A2.3.4 Balloon's coverage analysis in the study areas in the Andes and Amazon regions in Peru.}

For the planning of the balloon's flight route and study area coverage, we will use available stratospheric wind speed and direction data from [128], shown in 
Figure 61 and Figure 62. This data corresponds to the Pacific Missile Range Facility at Kauai (Hawaii) which is located at latitude $22.08^{\circ}$ north. The study [127] shows that wind speeds between latitudes $0^{\circ}-30^{\circ}$ north and $0^{\circ}-30^{\circ}$ south have a similar wind speed range from $0 \mathrm{~m} / \mathrm{s}$ to around $30 \mathrm{~m} / \mathrm{s}$ at $65 \mathrm{kft}$. Then for our analysis with study areas at latitude $13^{\circ}$ south, we will use this wind speed and direction data.

In October 2016, Project Loon announced that they conducted balloons flight tests over Peruvian airspace [113] [114]. Before this press release, since May 2016, we followed these fly tests over Peru using air traffic control (ATC) database websites [119] which recorded the flights of two Project Loon's balloons marked as HBAL273 and HBAL305. We used the flight pattern data collected from these ATC database websites to conduct our technical and engineering cost analyses following two defined Project Loon's balloons flight patterns:

\section{- Balloons flight around a fixed area to provide the broadband} service: Station-keeping functions should be implemented in the balloons to achieve this type of coverage. As mentioned earlier, the innovation of the Project Loon is to use steady stratospheric winds for propulsion for the balloons to move in a horizontal direction to a specific location or along a planned route. Project Loon conducted flight tests of balloons (HBAL273 and HBAL305) flying over the city of Chimbote in the northern coast of Peru.

- Balloons flight over a route in South America: Each balloon provides broadband service to fixed areas only for a period of time while client devices are in the base station antenna range. A constellation of a few hundred 
balloons are needed to provide continuous coverage to fixed locations. Project Loon conducted flight tests of a balloon (HBAL273) flying in a route over Peru, Bolivia, Paraguay, Brazil and then back to Bolivia and Peru). In recent press releases in February 2017, Project Loon stated that flight routes will keep balloons in much more concentrated locations over small loops over a land mass with 10 or 20 or 30 balloons, not with hundreds of balloons [131] [132] [133]. We used this recent approach in the technical and engineering cost analysis of the balloons flight over a route.

We analyze the both scenarios when balloons use the stratospheric winds to provide coverage to our two fixed study areas.

\section{Flight Route 1: Balloons flight around a fixed area to provide the broadband service}

In Figure 64, we used the wind profiles to estimate the stratospheric wind speeds and direction at altitudes between $50 \mathrm{kft}$ and $60 \mathrm{kft}$. Around $50 \mathrm{kft}$ and $60 \mathrm{kft}$, winds have opposite directions, $270^{\circ}$ and $90^{\circ}$ respectively, which can allow balloons to return to an initial route position. Then we will use the wind speed and direction at $50 \mathrm{kft}$ and $60 \mathrm{kft}$ for the balloon flight route. 

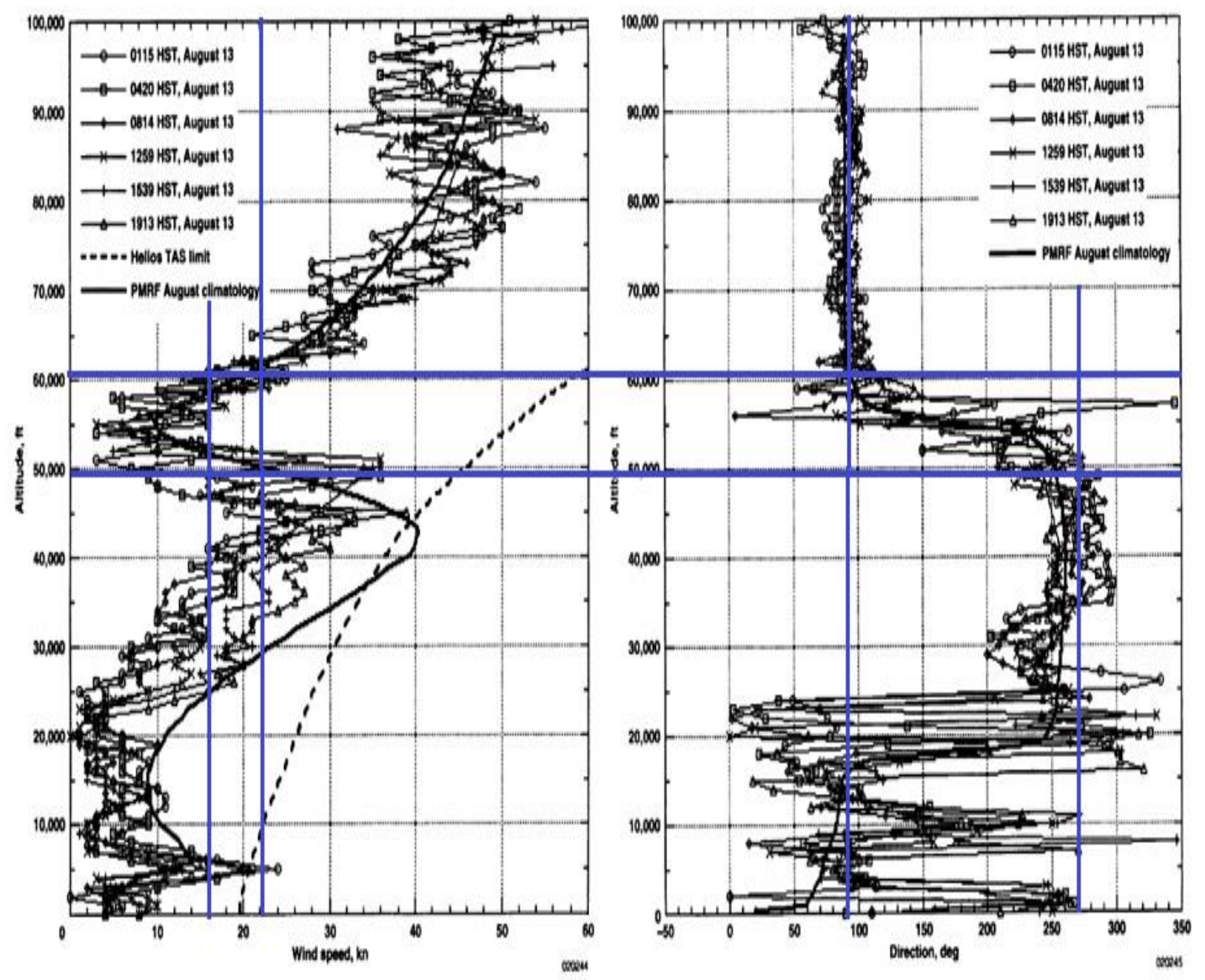

Figure 64. Estimation of wind speed and direction between $50 \mathrm{kft}$ and $60 \mathrm{kft}$.

Figure 65and Figure 66 show the tentative flight route over the Yauli and Samugari study areas based on the wind speed and direction profiles showed above. 


\section{Balloon Flight Route - Yauli}

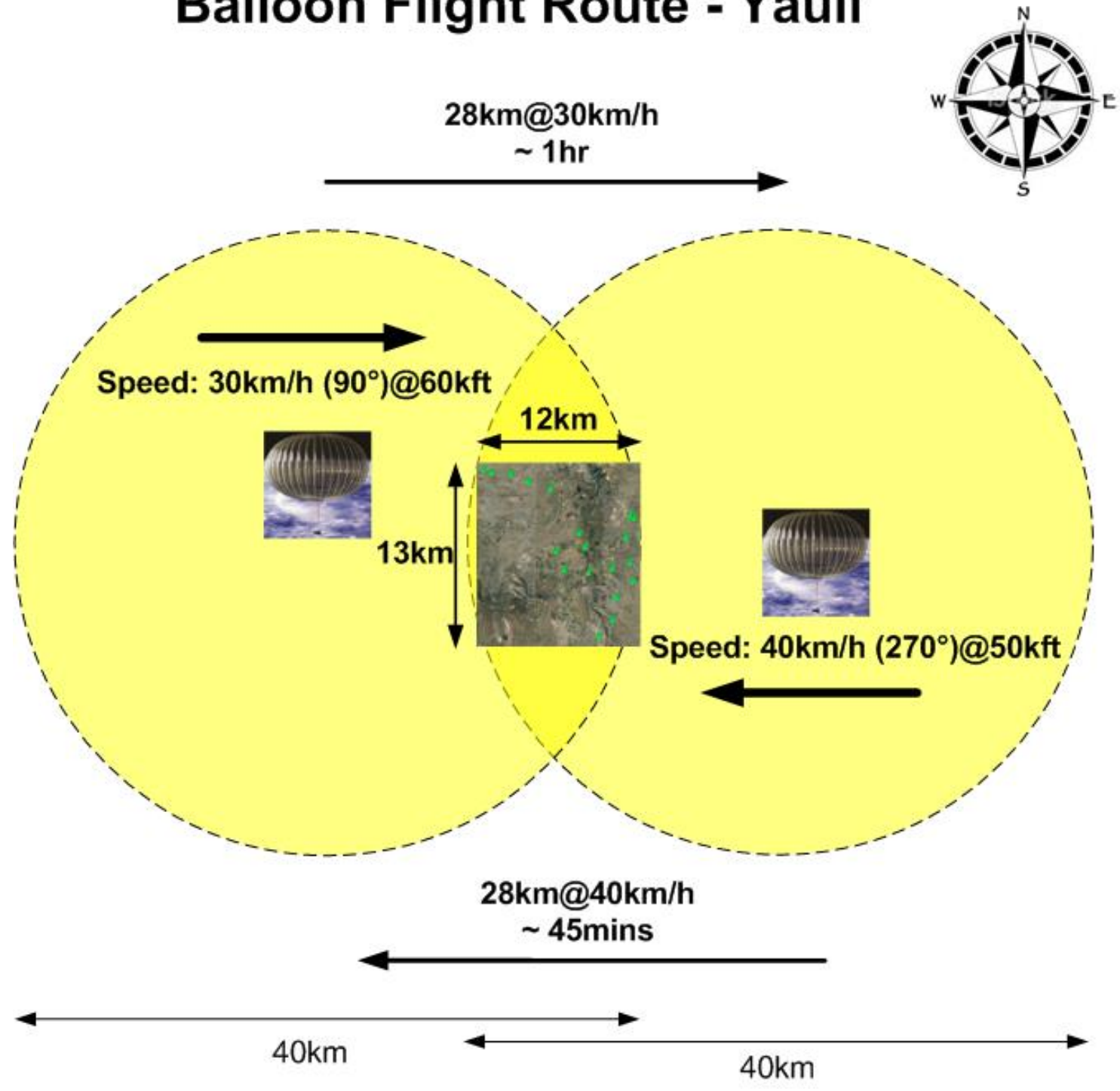

Figure 65. Balloon flight route over the Yauli study area. 


\section{Balloon Flight Route - Samugari}

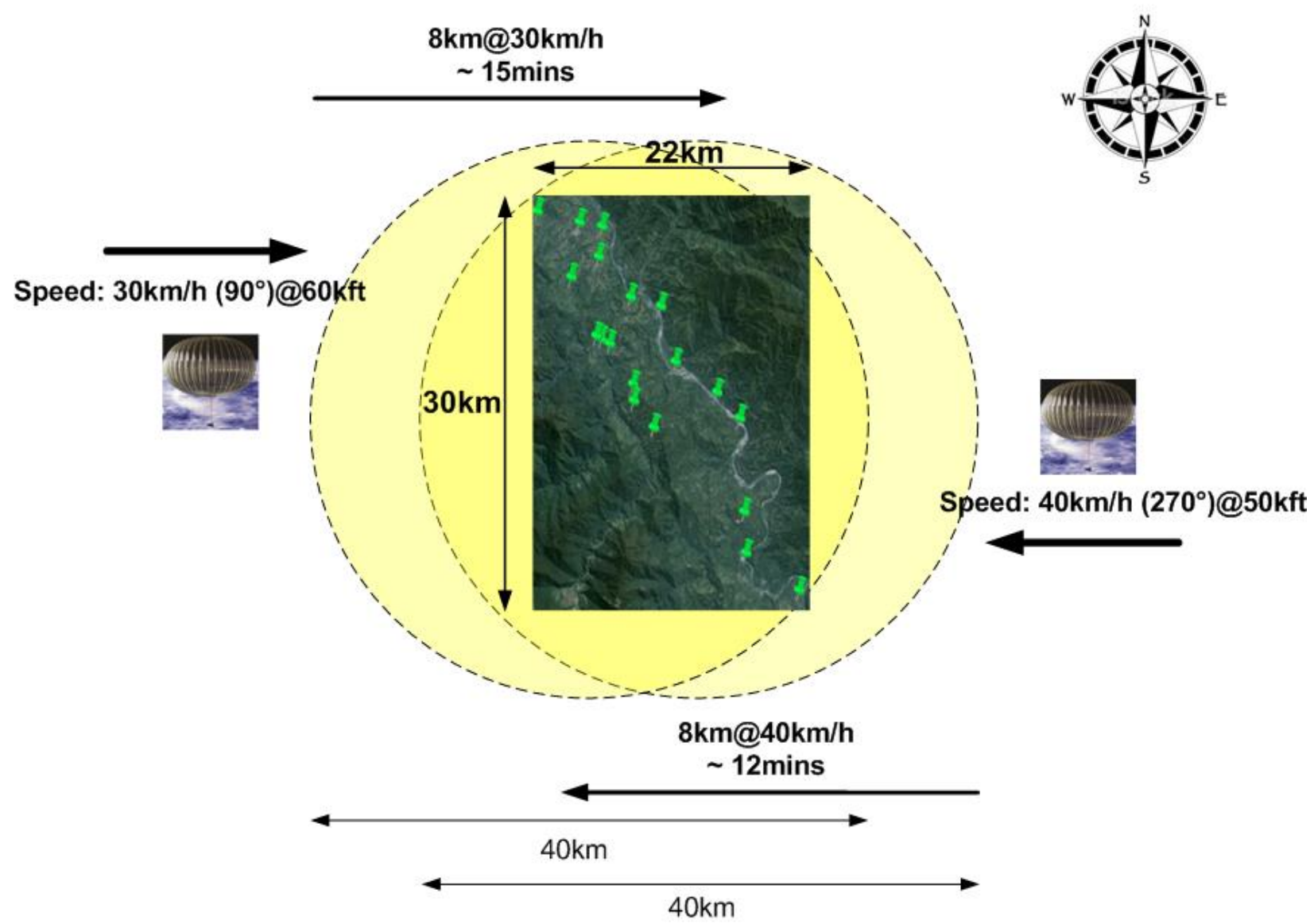

Figure 66. Balloon flight route over the Samugari study area

\section{Flight Route 2: Balloons flight over a route in South America}

Figure 67 shows a flight route test conducted by Google in South America and recorded in an Air Traffic Control website in [119]. This route shows that the balloon flew over the two study areas setting them as potential broadband service areas in the future. Based on this route flying over Peru, Bolivia, Paraguay, Brazil and then back to Bolivia and Peru, we estimated that 195 balloons would be required to provide permanent wireless broadband service over the two study 
areas. However, based on the recent announcements from Project Loon [131] [132] [133], that flight routes will keep balloons in much more concentrated locations over small loops over a land mass with a few tens of balloons, not with hundreds of balloons, we assumed smaller local routes over the two study areas. The updated smaller flight route requires 26 balloons to provide permanent wireless broadband service over the two study areas.

In Figure 68, we used the wind profiles to estimate the stratospheric wind speeds and direction at altitudes between $60 \mathrm{kft}$ and $80 \mathrm{kft}$. At these altitudes, winds have higher speeds than between $50 \mathrm{kft}$ and $60 \mathrm{kft}$. These high speed winds can be used to propel balloons at faster speeds over unpopulated areas or over the ocean. 


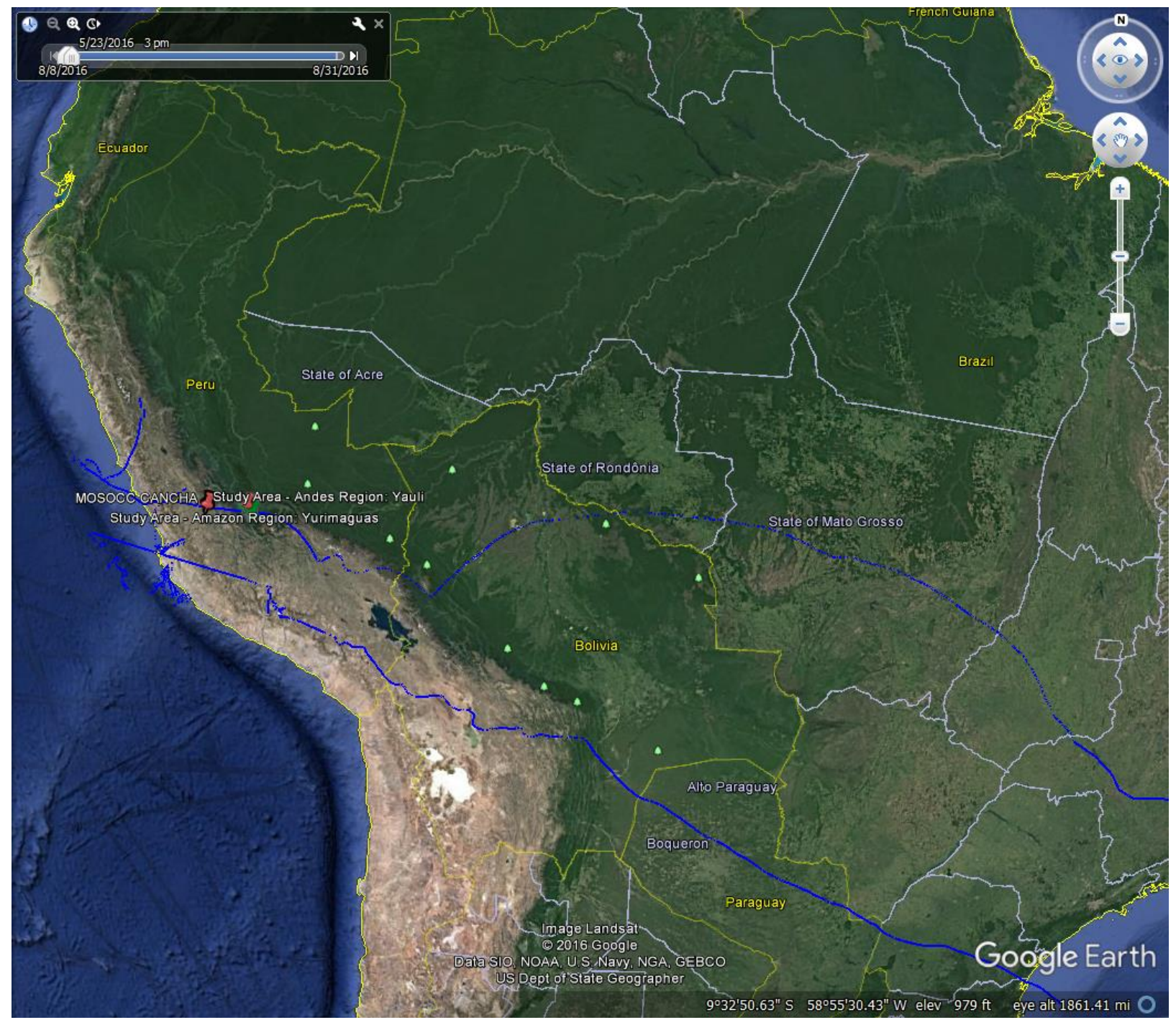

Figure 67. Balloon flight route test in South America 


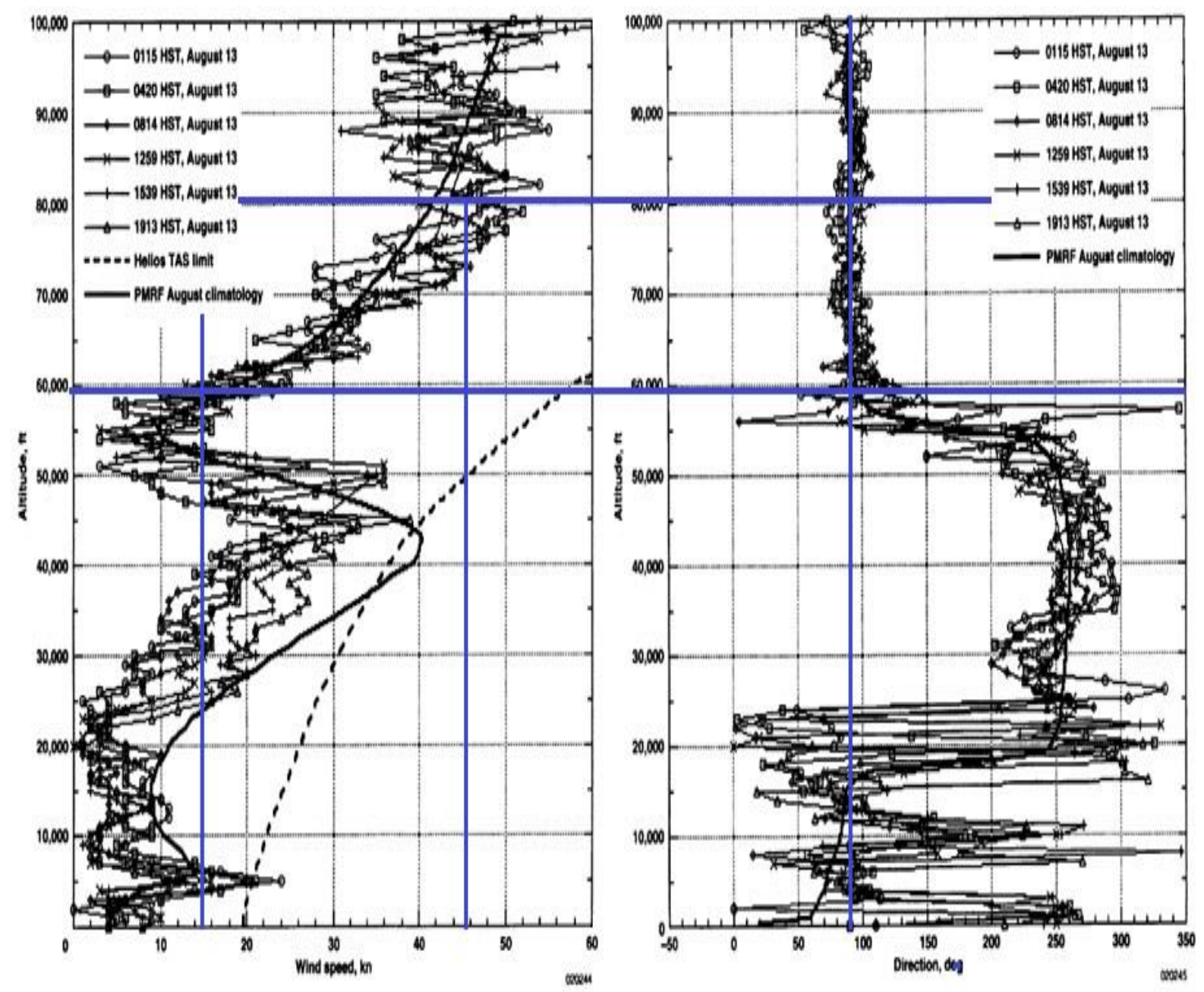

Figure 68 . Estimation of wind speed and direction between $60 \mathrm{kft}$ and $80 \mathrm{kft}$.

Figure 69 shows the tentative flight route over the Yauli study area based on the wind speed and direction profiles showed above. In this case, each balloon will continue flying after passing over the study area and will fly at higher speed over unpopulated areas or the ocean. After one balloon passes over the study area, another balloon will arrive. In this way, the broadband service coverage is provided in a permanent way. In Samugari, the balloons will have a similar flight pattern. 


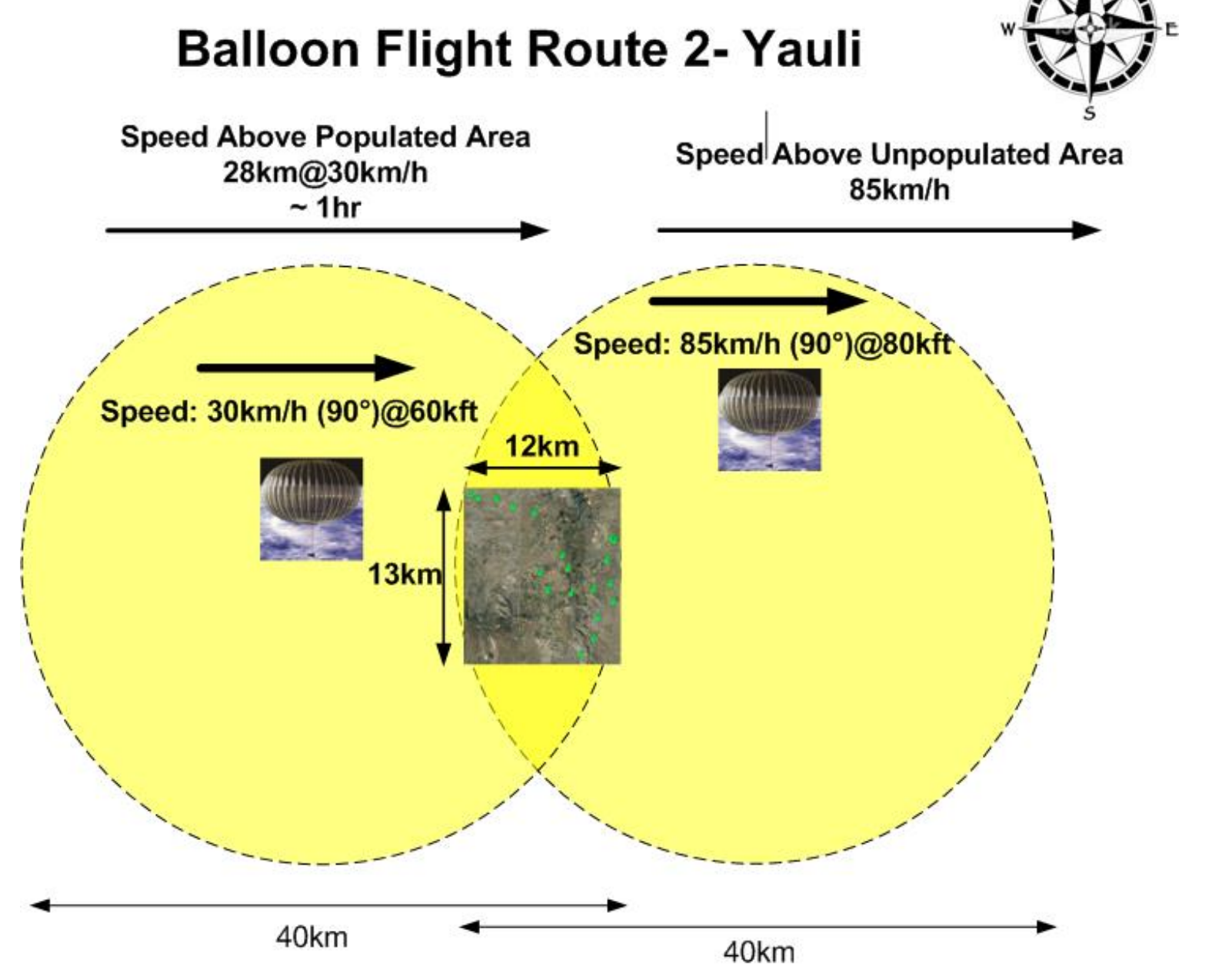

Figure 69. Balloon flight route over the Yauli study area for the case of a route in South America.

\section{A2.3.5 Balloon Cost}

According to Mike Cassidy, director of the Loon project, the cost of each balloon is of "tens of thousands of dollars" which is a fraction of the cost of a satellite (hundreds of millions to build and hundreds of millions to launch) [54]. Mike Cassidy also points that operating costs of the balloons are an order of magnitude or two cheaper to operate on a daily basis than satellite, even for a global network. It should be possible to operate on Loon balloon for just hundreds 
of dollars per day and each balloon should be able to serve a few thousand connections at any time.

Based on the envelope and payload elements mentioned in previous subsections, we conducted the following cost estimation for each balloon, shown in Table 22 . 
Table 22. Estimated cost of the balloon system including payload, envelope and assembly.

\begin{tabular}{|c|c|c|c|}
\hline Envelope \& Payload Items & Qty & Description & $\begin{array}{c}\text { Price } \\
\text { USD }\end{array}$ \\
\hline \multirow{2}{*}{ Computational Subsystem } & 1 & Processor & $\$ 280.00$ \\
\hline & 1 & On-board data storage & $\$ 40.00$ \\
\hline \multirow{2}{*}{ Solar Energy Subsystem } & 4 & Rechargeable battery & $\$ 1,932.00$ \\
\hline & 6 & Solar power generation (solar panels) & $\$ 5,040.00$ \\
\hline \multirow{3}{*}{ Navigation subsystem } & 1 & Navigation system & $\$ 3,075.00$ \\
\hline & 1 & Transponder & $\$ 2,250.00$ \\
\hline & 1 & VHF/UHF RF Control System & $\$ 3,000.00$ \\
\hline Sensing Subsystem & 1 & Environmental data probe & $\$ 119.20$ \\
\hline \multirow{4}{*}{$\begin{array}{l}\text { Telecom Subsystem 1: RF } \\
\text { Access Link To Client } \\
\text { Devices (approx 10Mbps) }\end{array}$} & 1 & RF transceiver & $\$ 21,240.00$ \\
\hline & 1 & RF Cable & $\$ 60.00$ \\
\hline & 1 & Antenna System & $\$ 176.77$ \\
\hline & 1 & Antenna Alignment Pointer & $\$ 1,600.00$ \\
\hline \multirow{4}{*}{$\begin{array}{l}\text { Telecom Subsystem 2: RF } \\
\text { Access Link To Backbone } \\
\text { (approx 10Mbps) }\end{array}$} & 1 & $\mathrm{RF}$ transceiver & $\$ 2,382.38$ \\
\hline & 1 & RF Cable & $\$ 0.00$ \\
\hline & 1 & Antenna System & $\$ 0.00$ \\
\hline & 1 & Antenna Alignment Pointer & $\$ 1,600.00$ \\
\hline \multirow{6}{*}{ Altitude Control Subsystem } & 1 & High-pressure storage chamber & $\$ 266.40$ \\
\hline & 1 & Gas turbine generator & $\$ 7.90$ \\
\hline & 1 & Gas pump & $\$ 45.00$ \\
\hline & 1 & Fuel cell & $\$ 134.10$ \\
\hline & 1 & Gas-flow tube & $\$ 20.00$ \\
\hline & 1 & Valve & $\$ 20.00$ \\
\hline Payload case & 2 & Weatherproof Case & $\$ 795.60$ \\
\hline Parachute & 1 & Recovery Parachute & $\$ 1,600.00$ \\
\hline Envelope & 1 & Super-pressure balloon envelope & $\$ 4,500.00$ \\
\hline Gas & 1 & Helium gas & $\$ 3,000.00$ \\
\hline \multirow[t]{4}{*}{$\begin{array}{l}\text { Other compenents, materials } \\
\& \text { accessories (uncertainty } \\
10 \% \text { ) }\end{array}$} & 1 & $15 \%$ of the rest of the balloon system & $\$ 3,918.78$ \\
\hline & & Subtotal & $\$ 57,103.13$ \\
\hline & & UAS Assembly \& Integration (15\%) & $\$ 8,565.47$ \\
\hline & & TOTAL COST & $\$ 65,668.60$ \\
\hline
\end{tabular}




\section{Appendix 3: Calculation of the Effective Download Channel Capacity Calculation}

This section describes the calculation of the effective channel capacity, also called average cell throughput, for the analyzed wireless technologies and presents the factors and variables used to carry out this calculation such as data rates and coverage area. The first subsection presents the maximum theoretical and actual data rates for the wireless technologies, and the second subsection presents the calculation of the effective channel capacity.

\section{A3.1 Maximum Theoretical and Actual Data Rate}

\section{Wi-Fi IEEE 802.11}

The Wi-Fi access network design criteria uses a green field scenario with only IEEE 802.11n client devices, not including legacy standards (IEEE 802.11a, $802.11 \mathrm{~b}$ or $802.11 \mathrm{~g})$. The $802.11 \mathrm{n}$ standard has a broad range of data rates from a few to hundreds of Mbps depending on modulation, coding schemes and number of spatial stream. The $802.11 \mathrm{n}$ standard achieve higher data rates than legacy standards such as $802.11 \mathrm{~b}$ (maximum rate of $11 \mathrm{Mbps}$ ) and $802.11 \mathrm{a} / \mathrm{g}$ (maximum rates of $54 \mathrm{Mbps}$ ) due to improvements in the physical layer (more OFDM carriers, maximum FEC rate, reduced guard interval, use of MIMO techniques, low density parity check, etc), and the Medium Access Control (MAC) layer (use of fragmentation and aggregation techniques, enhanced block acknowledgement, among other enhanced features) [23]. Using both 802.11n and legacy standards 
would lead to a decrease in the average channel capacity per AP due to allocating airtime to slower legacy client devices.

Table 23 shows maximum theoretical actual data rates of the standard IEEE 802.11n. Our wireless access network design uses 802.11n equipment with 2 streams (2x2 MIMO) for the access points and client devices, standard guard interval (GI) of $800 \mathrm{~ns}$, and bandwidth of $20 \mathrm{MHz}$ in order to maximize the number of non-interference channels in each node. The unlicensed 2.4GHz Wi-Fi band allows for 3 non-interference $20 \mathrm{MHz}$ channels or 1 non-interference $40 \mathrm{MHz}$ channel.

Table 23. IEEE 802.11n physical layer data rates [134] [135]

\begin{tabular}{|c|c|c|c|c|c|c|}
\hline \multirow{2}{*}{$\begin{array}{l}\text { MCS } \\
\text { Index }\end{array}$} & \multirow{2}{*}{ Modulation } & \multirow{2}{*}{\begin{tabular}{|l|} 
\# of \\
Spatial \\
Streams
\end{tabular}} & \multicolumn{2}{|c|}{$\mathrm{GI}=800 \mathrm{~ns}$} & \multicolumn{2}{|c|}{$\mathrm{GI}=400 \mathrm{~ns}$} \\
\hline & & & $20 \mathrm{MHz}$ & $40 \mathrm{MHz}$ & $20 \mathrm{MHz}$ & $40 \mathrm{MHz}$ \\
\hline 0 & BPSK $1 / 2$ & 1 & 6.5 & 13.5 & $72 / 9$ & 15 \\
\hline 1 & QPSK 1/2 & 1 & 13 & 27 & $144 / 9$ & 30 \\
\hline 2 & QPSK 3/4 & 1 & 19.5 & 40.5 & $212 / 3$ & 45 \\
\hline 3 & 16QAM 1/2 & 1 & 26 & 54 & $288 / 9$ & 60 \\
\hline 4 & 16QAM 3/4 & 1 & 39 & 81 & $431 / 3$ & 90 \\
\hline 5 & 64QAM $2 / 3$ & 1 & 52 & 108 & $577 / 9$ & 120 \\
\hline 6 & 64QAM 3/4 & 1 & 58.5 & 121.5 & 65 & 135 \\
\hline 7 & 64QAM 5/6 & 1 & 65 & 135 & $722 / 9$ & 157.5 \\
\hline 8 & BPSK 1/2 & 2 & 13 & 27 & $144 / 9$ & 30 \\
\hline 9 & QPSK 1/2 & 2 & 26 & 54 & $288 / 9$ & 60 \\
\hline 10 & QPSK 3/4 & 2 & 39 & 81 & $431 / 3$ & 90 \\
\hline 11 & 16QAM 1/2 & 2 & 52 & 108 & $577 / 9$ & 120 \\
\hline 12 & 16QAM 3/4 & 2 & 78 & 162 & $862 / 3$ & 180 \\
\hline 13 & 64QAM $2 / 3$ & 2 & 104 & 216 & $1155 / 9$ & 240 \\
\hline 14 & 64QAM 3/4 & 2 & 117 & 243 & 130 & 270 \\
\hline 15 & 64QAM 5/6 & 2 & 130 & 270 & $1444 / 9$ & 300 \\
\hline
\end{tabular}


For the calculation of the aggregated downlink channel capacity, we use the estimated actual data rates which are $60-70 \%$ [136] of the physical data rate due to the half-duplex nature of the medium and the overhead generated by the multiple access technique, carrier sense multiple access/collision avoidance (CSMA/CA), and including an additional 7\% overhead due to the RTS/CTS mechanism to avoid collisions [137]. This mechanism is enabled in the two rural scenarios due to the large separation (hundreds of meters to few kilometers) among client devices which could lead to collisions to communicate to the access point.

\section{Long Term Evolution (LTE)}

LTE has a broad range of data rates from less than $1 \mathrm{Mbps}$ to hundreds of Mbps depending on modulation and coding schemes, and number of spatial stream, among other parameters. Table 24 shows data rates for LTE for different number of spatial streams, modulation and coding schemes, and commercial LTE channel bandwidths. The data rates were calculated as shown in [23]. Our LTE network design uses equipment with 2 streams for the base stations and client devices, and bandwidth of $10 \mathrm{MHz}$ in order to obtain the best trade-off between channel reuse factor, coverage area and maximum number of clients per base station. This tradeoff was analyzed based on the current $1.7 / 2.1 \mathrm{GHz}$ LTE band regulation cap of $20 \mathrm{MHz}$ per operator. 
Table 24. LTE physical layer data rates

\begin{tabular}{|c|c|c|c|c|c|c|c|}
\hline & \multicolumn{6}{|c|}{ Channel Bandwidth } \\
\hline & & $1.4 \mathrm{MHz}$ & $3 \mathrm{MHz}$ & $\mathbf{5 M H z}$ & $\mathbf{1 0 M H z}$ & $15 \mathrm{MHz}$ & $20 \mathrm{MHz}$ \\
\hline & & \multicolumn{6}{|c|}{ Resource Blocks } \\
\hline Modulation & $\begin{array}{c}\text { \# of } \\
\text { Spatial } \\
\text { Streams }\end{array}$ & 6 & 15 & 25 & 50 & 75 & 100 \\
\hline QPSK 1/2 & 1 & 1 & 2.5 & 4.2 & 8.4 & 12.6 & 16.8 \\
\hline QPSK 3/4 & 1 & 1.5 & 3.75 & 6.3 & 12.6 & 18.9 & 25.2 \\
\hline 16QAM 1/2 & 1 & 2 & 5.05 & 8.4 & 16.8 & 25.2 & 33.6 \\
\hline 16QAM 3/4 & 1 & 3 & 7.575 & 12.6 & 25.2 & 37.8 & 50.4 \\
\hline 64QAM 2/3 & 1 & 4.07 & 10.07 & 16.8 & 33.6 & 50.4 & 67.2 \\
\hline 64QAM 3/4 & 1 & 4.58 & 11.33 & 18.9 & 37.8 & 56.7 & 75.6 \\
\hline QPSK 1/2 & 2 & 2 & 5.05 & 8.4 & 16.8 & 25.2 & 33.6 \\
\hline QPSK 3/4 & 2 & 3 & 7.575 & 12.6 & 25.2 & 37.8 & 50.4 \\
\hline 16QAM 1/2 & 2 & 4.00 & 10.10 & 16.8 & 33.6 & 50.4 & 67.2 \\
\hline 16QAM 3/4 & 2 & 6.00 & 15.15 & 25.2 & 50.4 & 75.6 & 100.8 \\
\hline 64QAM 2/3 & 2 & 8.07 & 20.13 & 33.6 & 67.2 & 100.8 & 134.4 \\
\hline 64QAM 3/4 & 2 & 9.08 & 22.65 & 37.8 & 75.6 & 113.4 & 151.2 \\
\hline
\end{tabular}

LTE actual data rates can be estimated based on that $20 \%$ of the physical data rate is reserved for control and reference signals, and around $9-10 \%$ of the layer 2 is reserved for transport block size restrictions and frame overhead [23].

\section{WiMAX 802.16d}

WiMAX has also a broad range of data rates from few to hundreds of Mbps depending on modulation and coding schemes, and number of spatial stream, among other parameters. Table 25 shows the maximum peak data rates for WiMAX $802.16 \mathrm{~d}$. We used the maximum physical data rates at $3.5 \mathrm{MHz}$ in [23] and at $5 \mathrm{MHz}$ in [138] to calculate rates at $7 \mathrm{MHz}, 10 \mathrm{MHz}$ and $20 \mathrm{MHz}$. Our WiMAX network design uses equipment with 2 streams for the base stations and client 
devices, and bandwidth of $10 \mathrm{MHz}$ in order to obtain the best trade-off between channel reuse factor, coverage area and maximum number of clients per base station. This trade-off was analyzed based on the current 3.5GHz WiMAX band regulation cap of $50 \mathrm{MHz}$ per operator.

Table 25. 802.16d Maximum Data Rates

\begin{tabular}{|c|c|c|c|c|c|c|}
\cline { 3 - 7 } \multicolumn{2}{c|}{} & \multicolumn{5}{c|}{ Channel Bandwidth } \\
\hline Modulation & $\begin{array}{c}\text { \# of } \\
\text { Spatial } \\
\text { Streams }\end{array}$ & $\mathbf{3 . 5 M H z}$ & $\mathbf{5 M H z}$ & $\mathbf{7 M H z}$ & $\mathbf{1 0 M H z}$ & $\mathbf{2 0 M H z}$ \\
\hline QPSK 1/2 & 1 & 2.82 & 3.95 & 5.64 & 7.9 & 15.8 \\
\hline QPSK 3/4 & 1 & 4.23 & 6 & 8.46 & 12 & 24 \\
\hline 16 QAM 1/2 & 1 & 5.64 & 8.06 & 11.28 & 16.12 & 32.24 \\
\hline 16 QAM 3/4 & 1 & 8.47 & 12.18 & 16.94 & 24.36 & 48.72 \\
\hline 64 QAM 2/3 & 1 & 11.29 & 16.30 & 22.58 & 32.6 & 65.2 \\
\hline 64 QAM 3/4 & 1 & 12.71 & 18.36 & 25.42 & 36.72 & 73.44 \\
\hline QPSK 1/2 & 2 & 5.64 & 7.9 & 11.28 & 15.8 & 31.6 \\
\hline QPSK 3/4 & 2 & 8.46 & 12 & 16.92 & 24 & 48 \\
\hline 16 QAM 1/2 & 2 & 11.28 & 16.12 & 22.56 & 32.24 & 64.48 \\
\hline 16 QAM 3/4 & 2 & 16.94 & 24.36 & 33.88 & 48.72 & 97.44 \\
\hline 64 QAM 2/3 & 2 & 22.58 & 32.6 & 45.16 & 65.2 & 130.4 \\
\hline 64 QAM 3/4 & 2 & 25.42 & 36.72 & 50.84 & 73.44 & 146.88 \\
\hline
\end{tabular}

Maximum theoretical data rates are quoted in standards or by manufacturers, but actual throughput vary with suppliers: a degradation of $40 \%$ to $50 \%$ is often observed [23].

\section{TVWS}

TVWS has data rates from two to twenty Mbps depending on modulation and coding schemes. Table 26 shows an example of the maximum Over the Air (OTA) data rates for a TVWS broadband system in a 6MHz channel (digital television channel bandwidth in Peru). 
Table 26. Maximum Over the Air (OTA) rates for a commercial broadband system [139].

\begin{tabular}{|c|c|c|}
\hline Modulation & $\begin{array}{c}\text { \# of } \\
\text { Spatial } \\
\text { Streams }\end{array}$ & $\mathbf{6} \mathbf{~ M H z}$ \\
\hline BPSK & 1 & 4 \\
\hline QPSK 1/2 & 1 & 4 \\
\hline QPSK 3/4 & 1 & 6 \\
\hline 16 QAM 1/2 & 1 & 8 \\
\hline 16 QAM 3/4 & 1 & 12 \\
\hline 16 QAM 2/3 & 1 & 16 \\
\hline
\end{tabular}

Actual data rates vary from $25 \%$ to $60 \%$ depending on the modulation rate and the distance [139].

\section{A3.2 Effective Channel Bandwidth}

For calculating the effective channel bandwidth, or average cell throughput, we use actual data rates and an estimation of users for each data rate [140] [141]. This calculation can be represented using the following formula:

$$
\text { Effective Channel Bandwidth }=\sum_{\text {Data Rates }}(\text { Data Rate } * \text { Data Rate Users })
$$

This formula is widely used to estimate effective channel bandwidth for LTE and WiMAX devices. We extend the use of this formula to Wi-Fi based on the assumption that access points enable the Airtime Fairness (or called Client Fairness by some manufacturers) function to distribute fairly the wireless network resources across clients. This function is commonly available in current commercial 802.11n access points [142] [143] [144]. For the case of TVWS equipment, network 
management functions are also available to ensure that all clients have equal access to network resources. These assumptions allow using the above formula to calculate the effective channel bandwidth for the analyzed technologies. Then the data rate user distribution at different data rates will be the main driver for the effective channel bandwidth.

The data rate user distribution is estimated based on the coverage area for the different data rates. The coverage area for each data rate is simulated using the software EDX. EDX calculates the coverage area for each data rate using the technical specifications of the access points, or base stations depending on the technology, and client devices, a propagation model and digital terrain data of the two study areas. Based on the simulated coverage areas for each data rate, we estimate the distribution or percentage of users for each data rate.

An important criterion for the wireless network design, for each analyzed technology, to maximize the effective channel capacity is using only outdoor client devices for communicating to the access point. These devices will use directional antennas pointing to the access points with higher gains than indoor devices use. The outdoor devices will connect to customer devices (PCs, laptops, etc.) using Ethernet cables. Indoor client devices are not included in the design to directly connect to the access point because it would lead to a reduction in the average channel capacity of each access point and to raise hidden nodes issues. This is due to, first, additional wall penetration losses, and second, lower antenna gains, which impacts the link budget and reduces the data rate of client devices even at 
locations close to the base stations. Furthermore, we run network design scenarios using indoor devices and then conducting the engineering cost analysis. The results showed that current FITEL bid node and infrastructure requirements outweigh the lower cost of indoor devices in comparison to outdoor devices. Then our design included only outdoor devices to maximize effective download channel capacity and reduce total network deployment and operation costs.

The actual data rates and the technical specifications associated to them in the EDX simulations will vary depending on the technology. For Wi-Fi, we will use the modulation schemes (BPSK, QPSK, 16QAM and 64QAM) with a robust forward error correction (FEC) coding scheme (1/2 for BPSK, QPSK amd 16QAM and 2/3 for 64QAM). This is due to using Wi-Fi for radio links at longer distances (up to a few kilometers) than it was designed for (up to a few hundred meters). For the case of LTE, WiMAX and TVWS, since these standards were designed for longer distances and wider coverage, we will use less robust FEC coding schemes. 


\section{Appendix 4: EDX Simulation Results for Yauli and Samugari.}

This appendix presents EDX simulation results for the LTE technology for the two study areas. Figure 70, Figure 71, Figure 72, Figure 73 show the EDX simulation results for the LTE technology in the Yauli area.

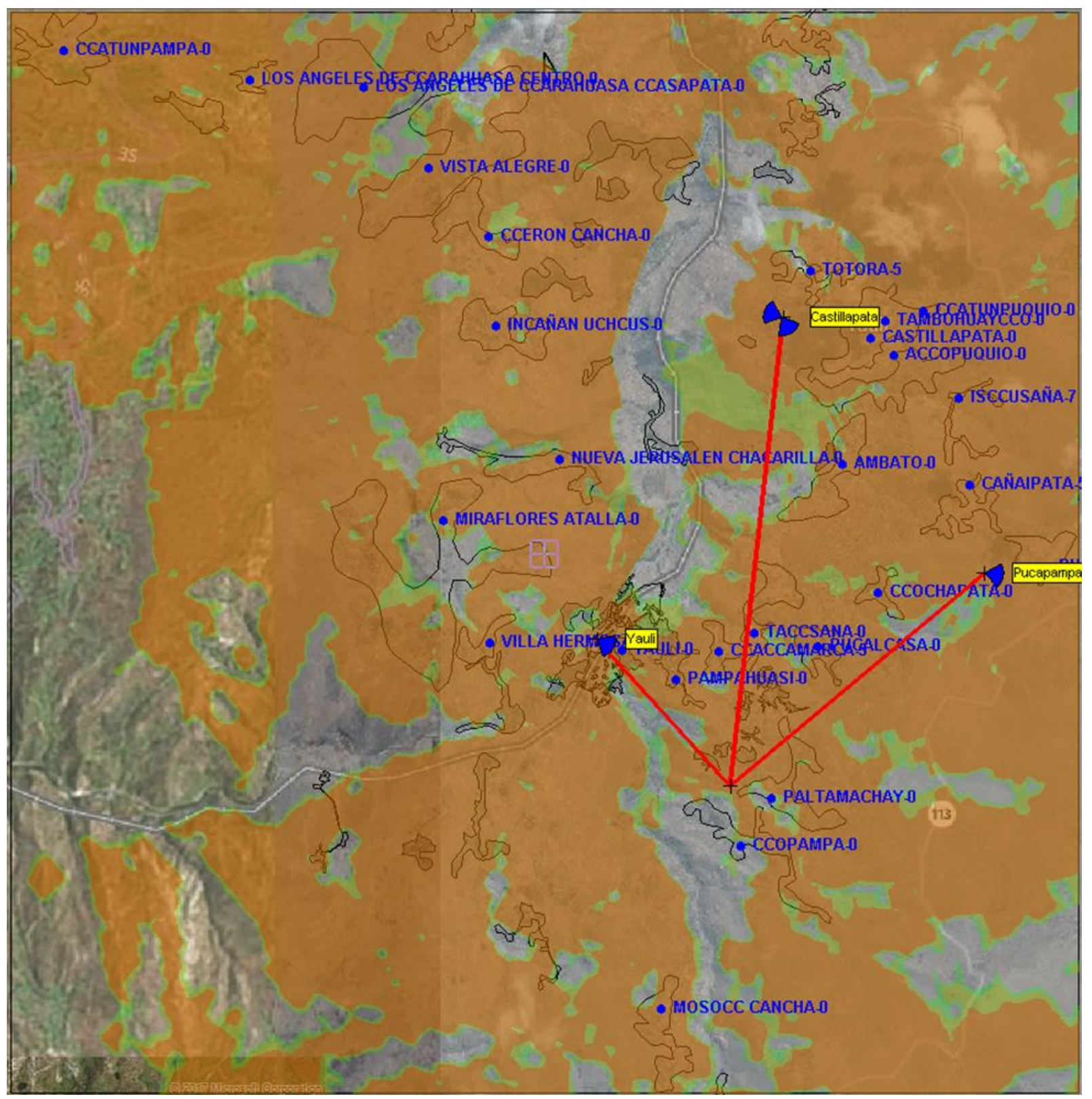

Figure 70. WBLAN using mini-LTE BSs for Yauli from year 1 to year 3. 


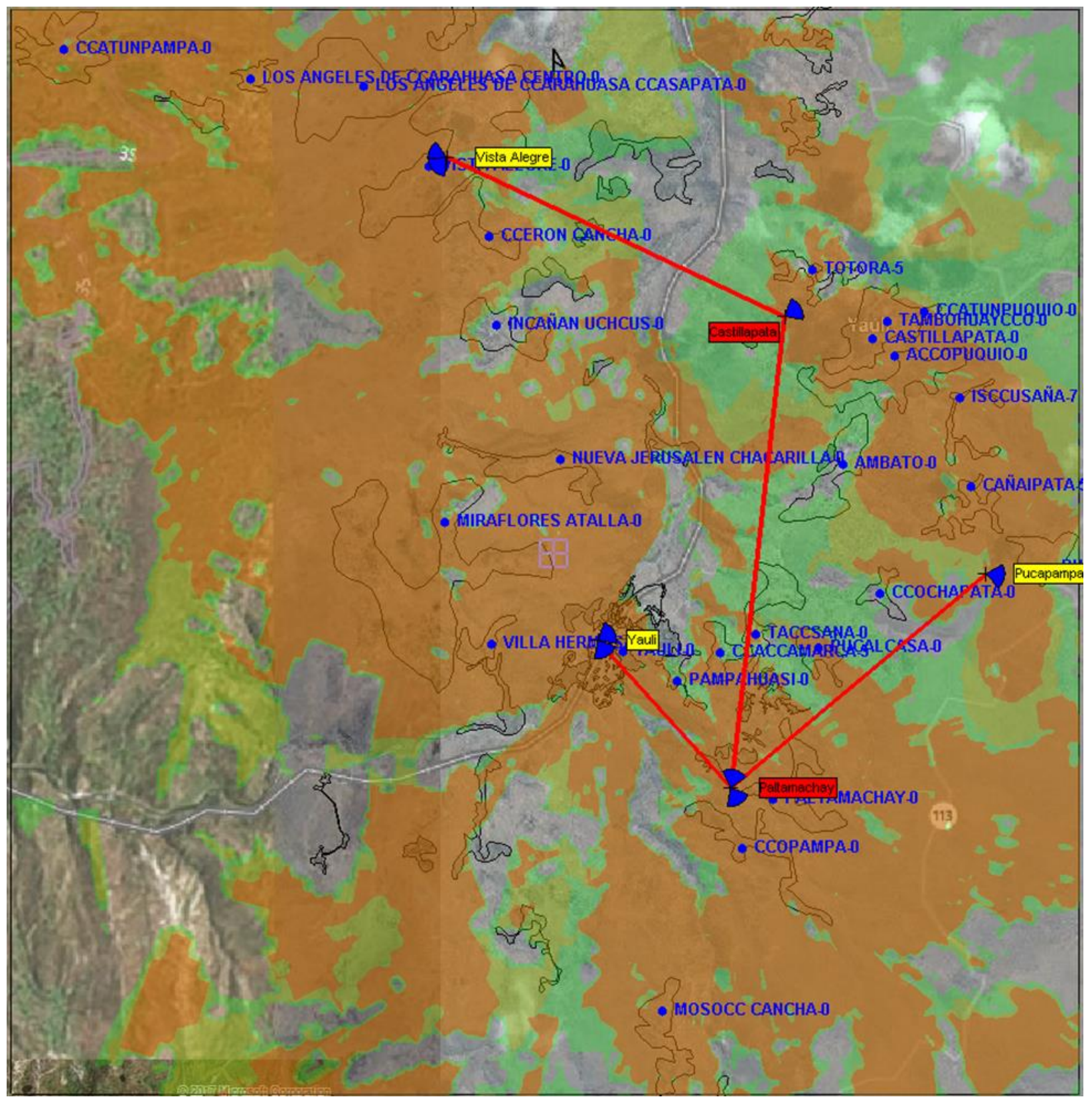

Figure 71. WBLAN using mini-LTE BSs for Yauli from year 4 to year 7 .

In years 8 to 10 , the average broadband speed demand per user is $8 \mathrm{Mbps}$ and 12Mbps, respectively. Then the WBLAN design excluded LTE's low modulation rates which could have slowed down the overall effective download channel capacity and node capacity, and additionally, generated co-channel 
interference when adding more nodes to the network. The same exclusion of low modulation rates for years 8 to 10 was applied to all the other technologies. Figure 72 and Figure 73 show the EDX simulation results for the LTE technology in the Yauli area, which in these cases do not include green coverage areas (low modulation rates) anymore.

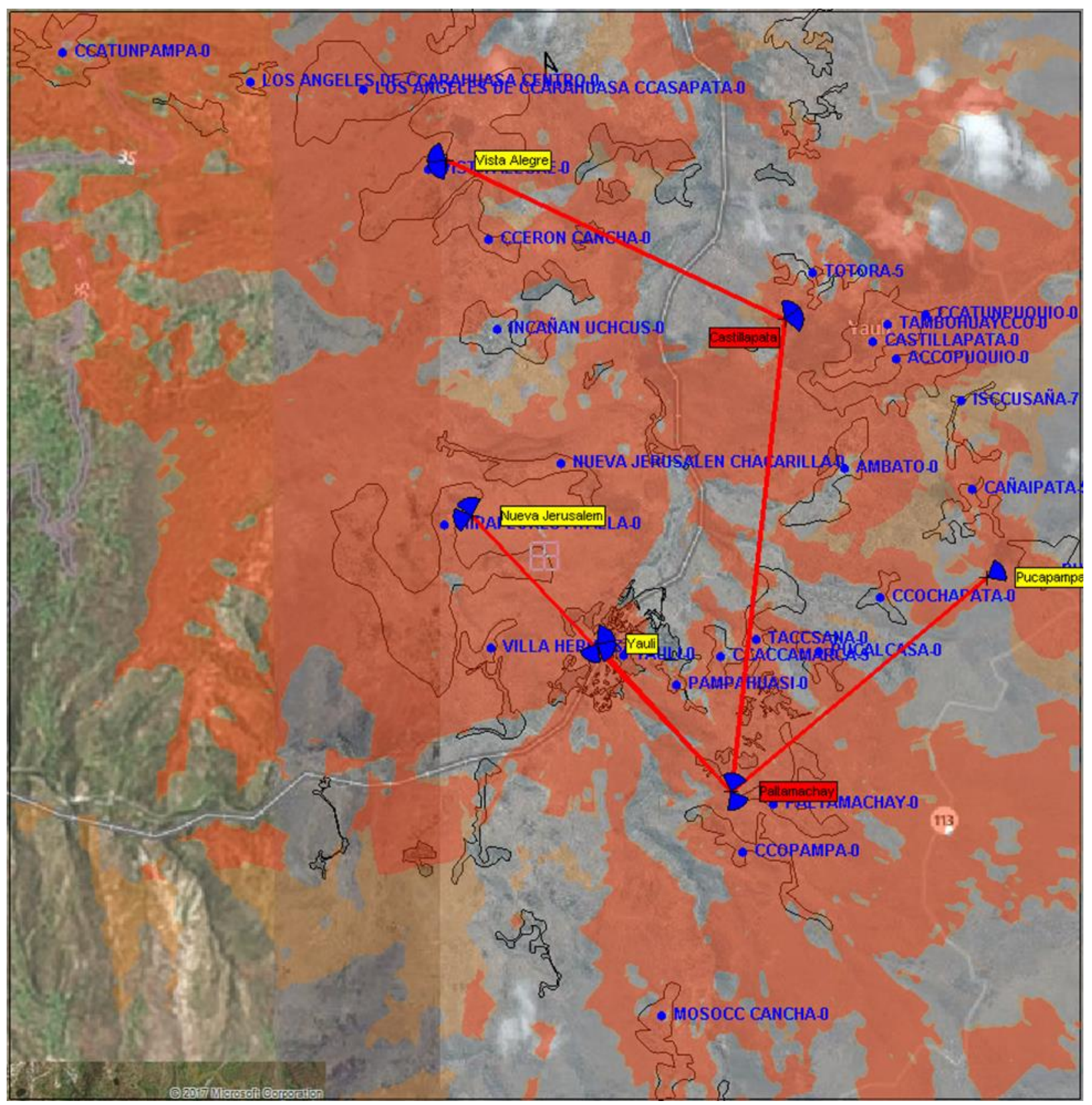

Figure 72. WBLAN using mini-LTE BSs for Yauli from year 8 to year 9. 


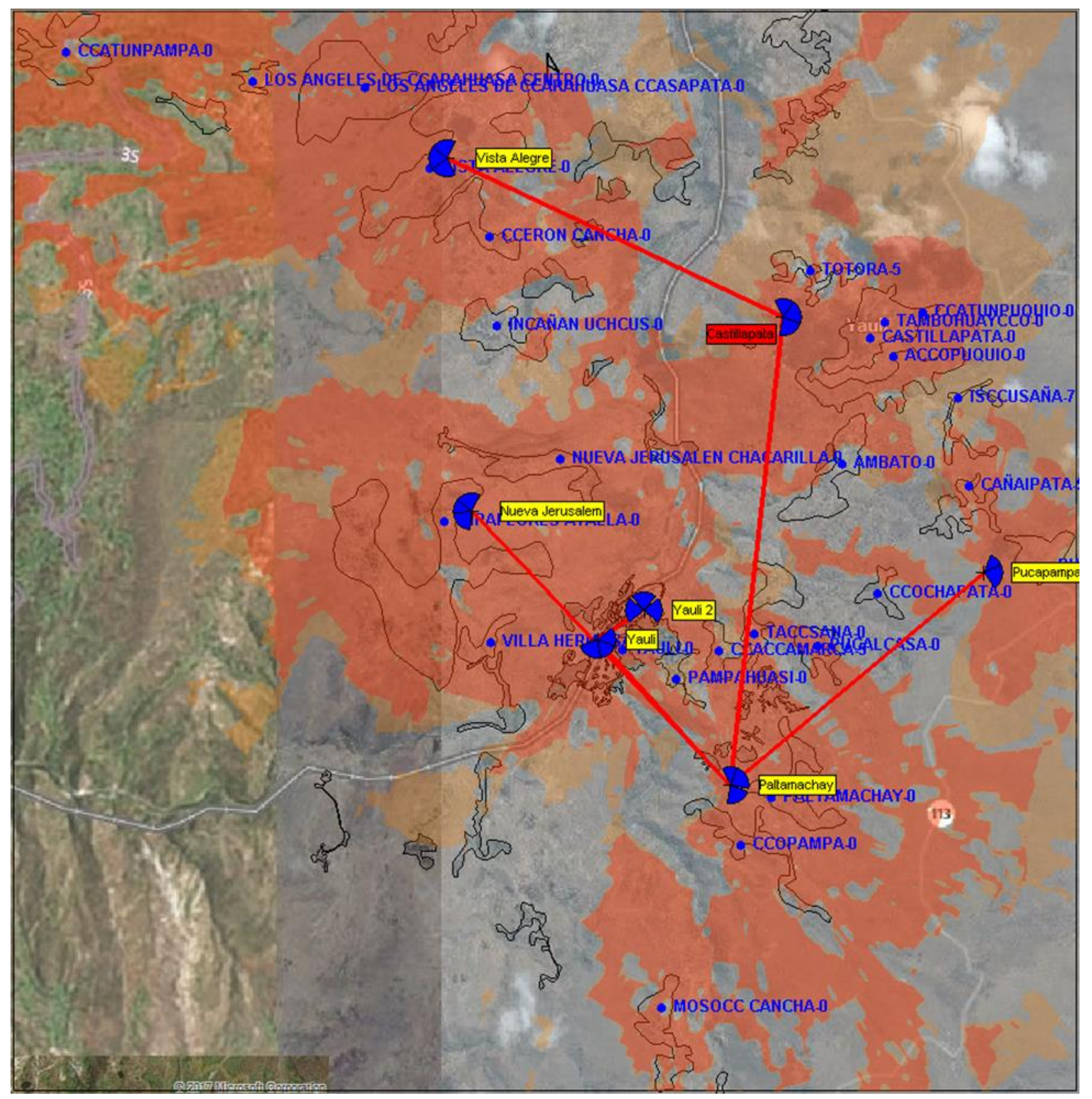

Figure 73. WBLAN using mini-LTE BSs for Yauli for year 10.

Figure 74, Figure 75 and Figure 76show the EDX simulation results for the LTE technology in the Samugari area. 


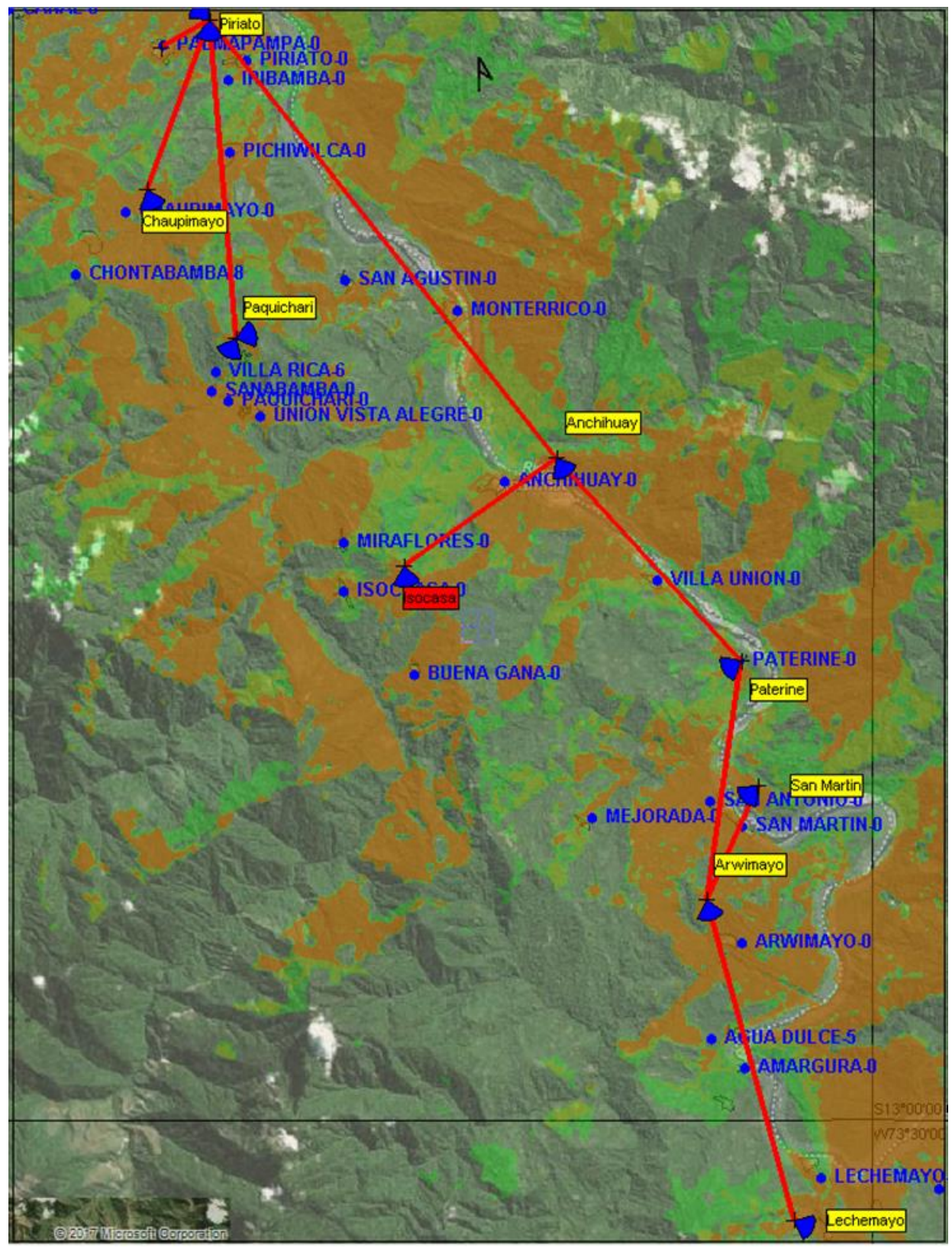

Figure 74. WBLAN using mini-LTE BSs for Samugari from year 1 to year 7 . 


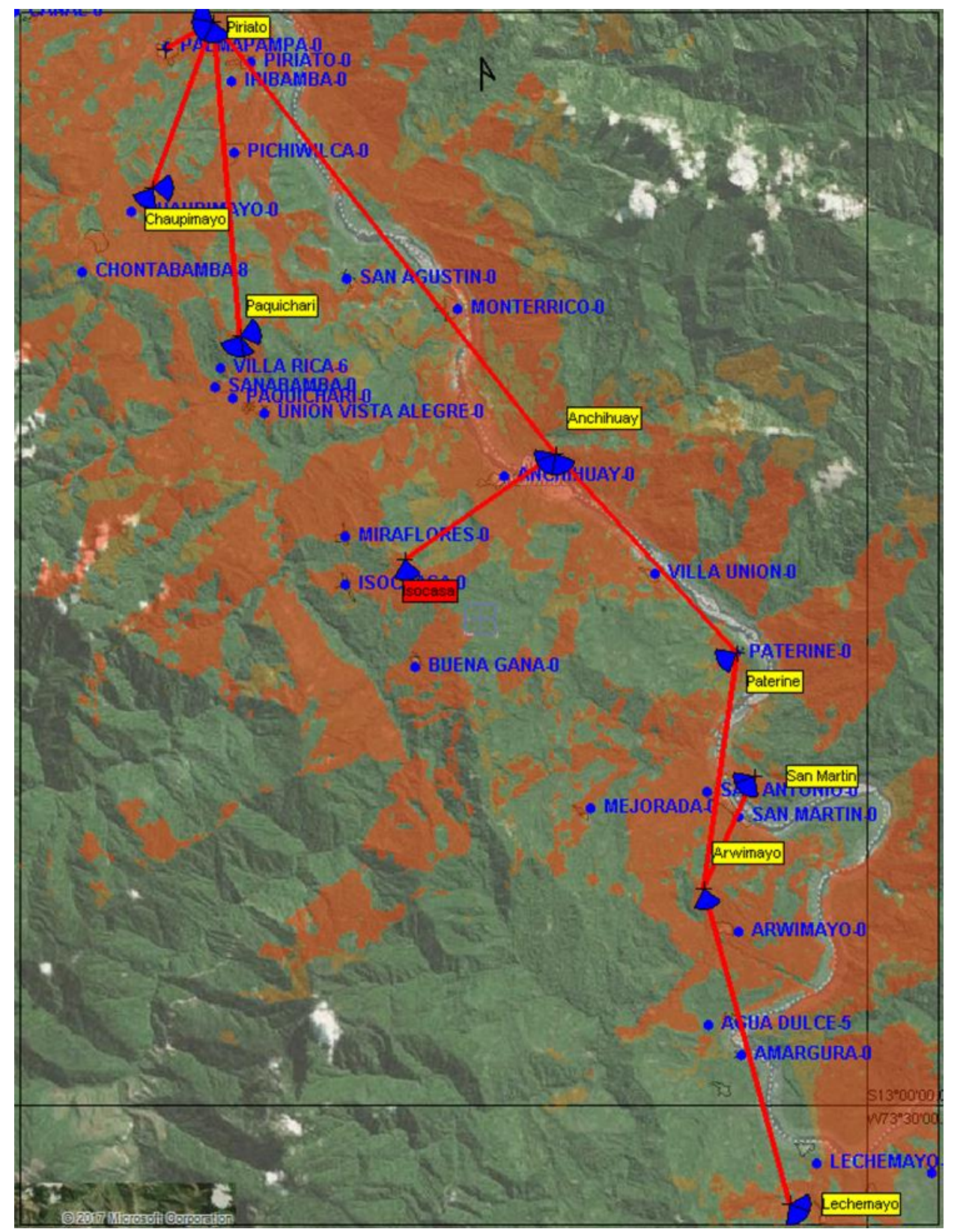

Figure 75. WBLAN using mini-LTE BSs for Samugari from year 8 to year 9 . 


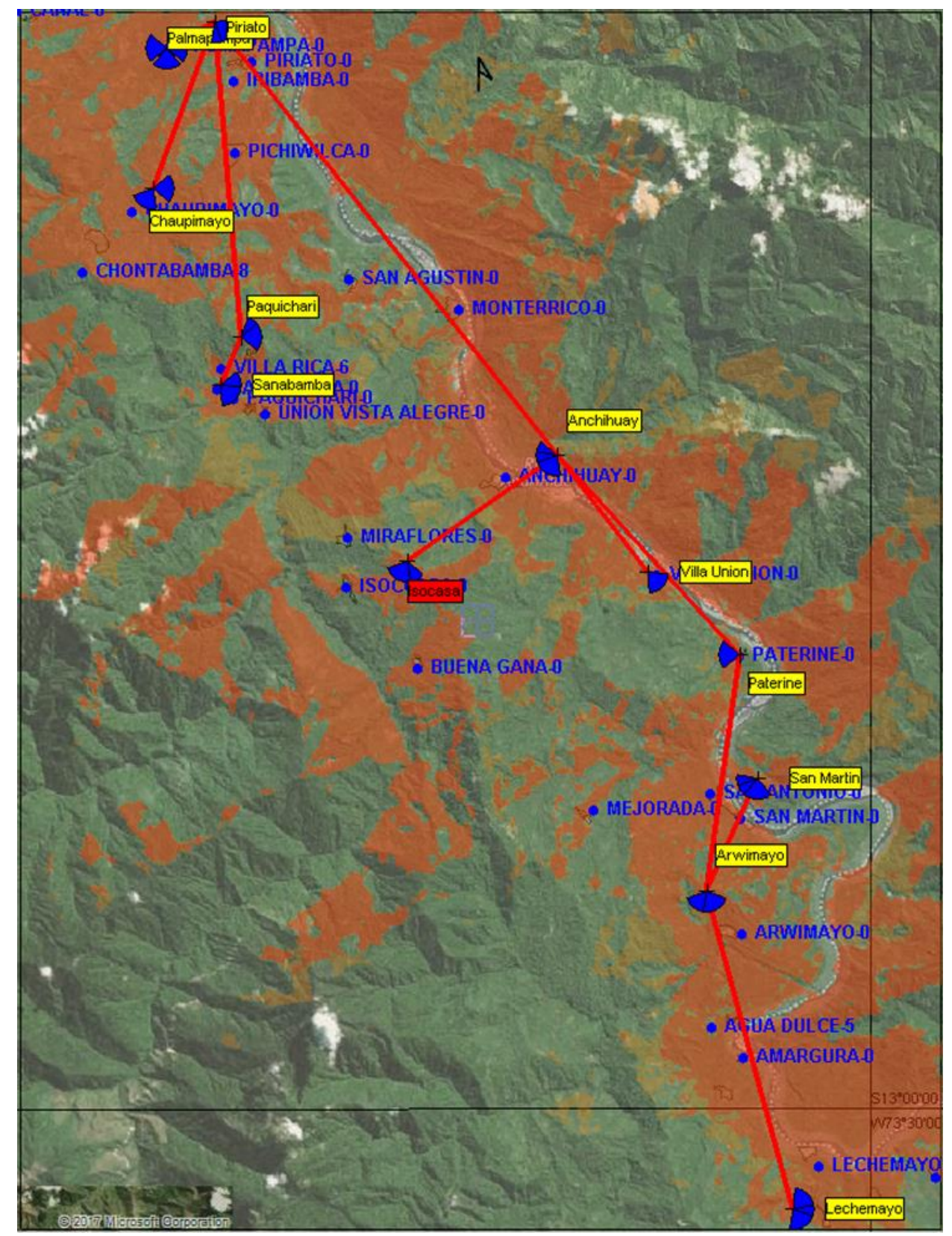

Figure 76. WBLAN using mini-LTE BSs for Samugari in year 10. 